\title{
Combined Time, Frequency and Space Diversity in Multimedia Mobile Broadcasting Systems
}

\author{
Departamento de Comunicaciones \\ Universitat Politècnica de València
}

A thesis for the degree of

Doctor en Telecomunicación

Valencia, June 2012

Author:

David Gozálvez Serrano

Supervisors:

Dr. David Gómez Barquero

Prof. Narcís Cardona Marcet 



\section{Abstract}

The combined use of diversity in the time, frequency, and space domains constitutes a powerful instrument to improve the reception of mobile broadcasting services. The improvement brought by the utilization of diversity techniques can be translated into an extended coverage of mobile services, or into a reduction of the network infrastructure. This dissertation addresses the use of diversity for the provision of mobile services in the European family of terrestrial broadcasting systems standardized by the DVB (Digital Video Broadcasting) consortium. This includes the first and second generation systems DVB-T (Terrestrial), DVB-H (Handheld) and DVB-T2 (Terrestrial 2nd Generation), as well as the next generation system DVB-NGH. Nevertheless, the work carried out in this dissertation is of generic nature and can be applied to future evolutions of standards such as the Japanese ISDB-T or the American ATSC. Our investigations employ an information-theoretic approach to obtain the performance limits of diversity techniques, as well as physical layer simulations to evaluate the performance in real systems.

The investigations carried out in the context of DVB-T, DVB-H, and DVBT2 are aimed at the simultaneous delivery of fixed and mobile services in terrestrial broadcasting networks. The convergence of the fixed and mobile paradigms can facilitate the introduction of mobile TV services by allowing the reuse of spectrum, content and infrastructure. The results show that the incorporation of time interleaving (TI) at the physical layer for time diversity, and single-input multiple-output (SIMO) for space diversity are critical for the performance of mobile broadcasting systems. Upper layer FEC (UL-FEC) techniques can be used to achieve time diversity in first generation systems like DVB-T and DVB-H; however, they require the transmission of additional parity data and are not useful for stationary reception. The analysis in terms of link budget reveals that the combined use of time and space diversity is not sufficient to enable the provision of mobile services with acceptable coverage levels in DVB-T and DVB-T2 networks planned for fixed reception. In contrast, diversity techniques can be used in networks planned for portable indoor 


\section{ABSTRACT}

reception to increase the capacity of vehicular services and extend the coverage of handheld indoor reception.

The utilization of combined diversity in the time, frequency, and space domains has been investigated in the context of DVB-NGH, the first broadcasting system to exploit the diversity in the three domains by incorporating at the physical layer long TI, time-frequency slicing (TFS) and multiple-input multiple-output (MIMO). In addition, the adoption of rotated constellations provides better robustness against fading by means of signal-space diversity (SSD).

DVB-NGH features an optional satellite component, and has adopted long TI in order to cope with the signal outages that are characteristic of land mobile satellite (LMS) channels. However, the solution adopted in the standard for long TI is cumbersome due to the poor performance exhibited by LDPC codes in the presence of heavy puncturing (erasures). In this context, we show that a split FEC approach at the physical layer can provide a good compromise in terms of time diversity (robustness) and zapping time.

TFS achieves better frequency diversity by grouping multiple RF channels in the same multiplex, so that the frequency interleaving can be performed across hundreds of $\mathrm{MHz}$ in the UHF band. According to the results presented in this dissertation, the utilization of TFS can achieve very significant gains depending on the maximum separation between RF channels.

The incorporation of MIMO in mobile broadcasting systems is key to overcome the Shannon limit of single antenna communications. In this regard, we focus on two important aspects when evaluating the performance of MIMO techniques in DVB-NGH: the transmission of pilots for channel estimation purposes, and the use of powerful FEC codes. Our investigations reveal that very simple techniques can outperform more complex codes with a priori better properties if the capacity loss due to the transmission of pilot information and the error-correcting capabilities of the FEC code are taken into account.

Broadly speaking, the additional diversity introduced by rotated constellations improves the performance of bit-interleaved coded modulation (BICM) for higher code rates, whereas for low code rates, it is preferable to rely on the error-correction capabilities of the FEC code. In this dissertation we show that the combination of rotated constellations with long TI and TFS is very interesting for mobile broadcasting systems. In particular, rotated constellations are important to increase the diversity gains in the time and frequency domains as well as to reduce the zapping time. 


\section{Resumen}

El uso combinado de diversidad en el dominio temporal, frecuencial, y espacial constituye una valiosa herramienta para mejorar la recepción de servicios de difusión móviles. Gracias a la mejora conseguida por las técnicas de diversidad es posible extender la cobertura de los servicios móviles además de reducir la infraestructura de red. La presente tesis investiga el uso de técnicas de diversidad para la provisión de servicios móviles en la familia europea de sistemas de difusión terrestres estandarizada por el consorcio DVB (Digital Video Broadcasting). Esto incluye la primera y segunda generación de sistemas DVB-T (Terrestrial), DVB-H (Handheld) y DVB-T2 (Terrestrial 2nd Generation), así como el sistema de siguiente generación DVB-NGH. No obstante, el estudio llevado a cabo en la tesis es genérico y puede aplicarse a futuras evoluciones de estándares como el japonés ISDB-T o el americano ATSC. Nuestra investigación emplea un enfoque basado en teoría de la información para obtener el máximo rendimiento de las técnicas de diversidad, así como simulaciones de capa física para evaluar su rendimiento en sistemas reales.

Las investigaciones realizadas dentro del contexto de DVB-T, DVB-H, y DVBT2 tienen como objetivo la transmisión simultánea de servicios fijos y móviles en redes terrestres de difusión. Esta convergencia puede facilitar la introducción de servicios móviles de TV debido a la reutilización de espectro, contenido e infraestructura. De acuerdo a los resultados, la incorporación de entrelazado de capa física para diversidad temporal, y de single-input multipleoutput (SIMO) para diversidad espacial, son esenciales para el rendimiento de sistemas de difusión móviles. A pesar de que las técnicas upper layer FEC (UL-FEC) pueden proporcionar diversidad temporal en sistemas de primera generación como DVB-T y DVB-H, éstas requieren la transmisión de paridad adicional y no son útiles para recepción estática. El análisis en términos de balance de enlace revela que las técnicas de diversidad no son suficientes para facilitar la provisión de servicios móviles en redes DVB-T y DVB-T2 planificadas para recepción fija. Sin embargo, el uso de diversidad en redes planificadas para la recepción de terminales portátiles en interiores puede mejorar 


\section{RESUMEN}

la capacidad de servicios a vehículos y posibilitar una buena cobertura para terminales de mano.

El uso combinado de diversidad en el dominio temporal, frecuencial, y espacial se ha investigado dentro de DVB-NGH, el primer sistema de difusión en incorporar los tres tipos de diversidad mediante entrelazado temporal de larga duración, time-frequency slicing (TFS) y multiple-input multiple-output (MIMO). Así mismo, la incorporación de constelaciones rotadas mejora la robustez frente a desvanecimientos por medio de signal-space diversity (SSD).

DVB-NGH incluye una componente satélite opcional, y por ello, ha adoptado entrelazado temporal de larga duración de forma que sea posible corregir los desvanecimientos que caracterizan los canales móviles satelitales. Sin embargo, la solución que se ha adoptado en el estándar para entrelazado temporal de larga duración presenta algunos problemas debido al mal rendimiento de los códigos LDPC frente a borrados. En esta tesis mostramos cómo una solución con doble FEC en la capa física puede proporcionar un buen compromiso entre diversidad temporal (robustez) y tiempo de zapping.

TFS mejora la diversidad en frecuencia agrupando múltiples canales RF en un mismo múltiplex, de forma que el entrelazado frecuencial pueda extenderse a lo largo de varios canales RF en la banda UHF. De acuerdo a nuestros resultados, el empleo de TFS puede proporcionar ganancias muy significantivas dependiendo de la separación máxima entre canales RF.

La incorporación de MIMO es clave para superar el límite de Shannon en sistemas con una única antena. En este sentido, nuestra investigación se centra en dos aspectos fundamentales a la hora de evaluar el rendimiento de técnicas MIMO en DVB-NGH: la transmisión de pilotos para la estimación del canal, y el uso de códigos FEC robustos. Los resultados de simulación revelan que técnicas muy simples pueden superar el rendimiento de códigos más complejos y con a priori mejores características, si se tiene en cuenta la pérdida de capacidad debida a la transmisión de pilotos así como las propiedades correctoras de los códigos FEC.

En general, la diversidad introducida por las constelaciones rotadas mejora el rendimiento de sistemas basados en bit-interleaved coded modulation (BICM) para tasas de codificación altas, mientras que para tasas de codificación bajas es preferible confiar en las propiedades correctoras del código FEC. En esta tesis mostramos que la combinación de constelaciones rotadas con entrelazado temporal de larga duración y TFS es muy interesante para los sistemas móviles de difusión. En concreto, las constelaciones rotadas son importantes para mejorar la diversidad en tiempo y en frecuencia así como para reducir el tiempo de zapping. 


\section{Resum}

L'ús combinat de diversitat en el domini temporal, freqüencial, i espacial constitueix una valuosa eina per millorar la recepció de serveis de difusió mòbils. Gràcies a la millora aconseguida per les tècniques de diversitat és possible estendre la cobertura dels serveis mòbils a més de reduir la infraestructura de xarxa. Aquesta tesi investiga l'ús de tècniques de diversitat per a la provisió de serveis mòbils a la família europea de sistemes de difusió terrestres estandarditzada pel consorci DVB (Digital Video Broadcasting). Això inclou la primera i segona generació de sistemes DVB-T (Terrestrial), DVB-H (Handheld) i DVB-T2 (Terrestrial 2nd Generation), així com el sistema de següent generació DVB-NGH. No obstant això, l'estudi dut a terme en la tesi és genèric i es pot aplicar a futures evolucions d'estàndards com el japonès ISDB-T o l'americà ATSC. La nostra recerca utilitza un enfocament basat en teoria de la informació per obtenir el màxim rendiment de les tècniques de diversitat, així com simulacions de capa física per avaluar el seu rendiment en sistemes reals.

Les investigacions realitzades dins del context de DVB-T, DVB-H, i DVBT2 tenen com a objectiu la transmissió simultània de serveis fixos i mòbils a xarxes terrestres de difusió. Aquesta convergència pot facilitar la introducció de serveis mòbils de TV a causa de la reutilització d'espectre, contingut i infraestructura. D'acord als resultats, la incorporació d'entrellaçat a la capa física per diversitat temporal, i de single-input multiple-output (SIMO) per diversitat espacial, són essencials per al rendiment de sistemes mòbils de difusió. Tot i que les tècniques upper layer FEC (UL-FEC) poden proporcionar diversitat temporal en sistemes de primera generació com DVB-T i DVB-H, requereixen la transmissió de paritat addicional i no són útils per a recepció estàtica. L'anàlisi en termes de balanç d'enllaç revela que les tècniques de diversitat no són suficients per facilitar la provisió de serveis mòbils en xarxes DVB-T i DVB-T2 planificades per a recepció fixa. No obstant això, l'ús de diversitat en xarxes planificades per a la recepció de terminals portàtils en interiors pot millorar la capacitat de serveis a vehicles i possibilitar una bona cobertura per a terminals de mà. 
L'ús combinat de diversitat en el domini temporal, freqüencial, i espacial s'ha investigat dins de DVB-NGH, el primer sistema de difusió en incorporar els tres tipus de diversitat mitjançant entrellaçat temporal de llarga durada, time-frequency slicing (TFS) i multiple-input multiple-output (MIMO). Així mateix, la incorporació de constel-lacions rotades millora la robustesa enfront d'esvaïments mitjançant signal-space diversity (SSD).

DVB-NGH inclou una component satèl-lit opcional, i per això, ha adoptat entrellaçat temporal de llarga durada de manera que sigui possible corregir els esvaïments que caracteritzen els canals mòbils satel-litals. No obstant això, la solució que s'ha adoptat en l'estàndard per entrellaçat temporal de llarga durada presenta alguns problemes a causa del mal rendiment dels codis LDPC davant esborrats. En aquesta tesi vam mostrar com una solució amb doble FEC a la capa física pot proporcionar un bon compromís entre diversitat temporal (robustesa) i temps de zàping.

TFS millora la diversitat en freqüència agrupant múltiples canals RF en un mateix múltiplex, de manera que l'entrellaçat freqüencial es pugui expandir a través de diversos canals RF a la banda UHF. D'acord als nostres resultats, l'ús de TFS pot proporcionar guanys molt significantius depenent de la separació màxima entre canals RF.

La incorporació de MIMO és clau per superar el límit de Shannon en sistemes amb una única antena. En aquest sentit, la nostra investigació se centra en dos aspectes fonamentals a l'hora d'avaluar el rendiment de tècniques MIMO en DVB-NGH: la transmissió de pilots per a l'estimació del canal, i l'ús de codis FEC robustos. Els resultats de simulació revelen que tècniques molt simples poden superar el rendiment de codis més complexos i amb a priori millors característiques, si es té en compte la pèrdua de capacitat deguda a la transmissió de pilots així com les propietats correctores dels codis FEC.

En general, la diversitat introduïda per les constel-lacions rotades millora el rendiment de sistemes basats en bit-interleaved coded modulation (BICM) per taxes de codificació altes, mentre que per a taxes de codificació baixes és preferible confiar en les propietats correctores del codi FEC. En aquesta tesi mostrem que la combinació de constel-lacions rotades amb entrellaçat temporal de llarga durada i TFS és molt interessant per als sistemes mòbils de difusió. En concret, les constel-lacions rotades són importants per millorar la diversitat en temps i en freqüència així com per reduir el temps de zàping. 


\section{Acknowledgements}

First of all, I feel obliged to acknowledge the Spanish Ministry of Industry, Tourism and Commerce for funding the research of this Thesis trough an official $\mathrm{PhD}$ scolarship. But, above and beyond that, my deepest gratitude is to my two advisors. Prof. Narcís Cardona gave me the opportunity to pursuit my $\mathrm{PhD}$ and be part of the Mobile Communications Group (MCG) and the Instituto de Comunicaciones y Aplicaciones Multimedia (iTEAM) at the Universitat Politècnica de València (UPV). Dr. David Gómez Barquero provided me with guidance and encouragement despite his many other academic and professional commitments. His advice was essential to the completion of this dissertation and has taught me innumerable lessons and insights on academic research. It is also my pleasure to thank Dr. Jarkko Paavola (University of Turku, Finland) and Dr. Thomas Stockhammer (NOMOR research, Germany) for inviting me to their research groups. This granted me the unique opportunity to gain a wider breadth of experience while still a graduate student.

Working in the MCG has been one of the best experiences in my life. Over the years, the people that had been part of the group have helped me to grow as a professional and as a person. I am specially grateful to Dr. José F. Monserrat, for his wisdom and counsel; to Dr. Daniel Calabuig, for so many enlightening discussions; and to Ariana, for her kindness and support. I'm also indebted to my colleagues and friends Ana, Gema, Paula, Pablo, Fernando, Jaime, Pedro, Carlos, Jordi, David, Jordi Joan, Guillermo, Pepe and Charlie for creating an excellent working environment and for enjoying all kinds of entertainment together. I would also like to thank the people in the Audio and Communications Signal Processing Group (GTAC) for their friendliness both inside and outside the university. Very special thanks go to Sandra for her understanding and trust.

$Y$ finalmente, quiero dar las gracias a mi familia. Ellos han sufrido como nadie el esfuerzo y dedicación necesarios para la realización de esta Tesis y me han demostrado su apoyo incondicional en los momentos más difíciles. Sin duda, ésta es también su tesis. 


\section{Table of contents}

List of Abbreviations $\quad$ xiii

1 Introduction $\quad 1$

1.1 Background ..................... 1

1.2 Problem Formulation . . . . . . . . . . . . . . . . . 9

1.3 Objectives and Thesis Scope . . . . . . . . . . . . . 10

1.4 Related Work . . . . . . . . . . . . . . . . . . 11

1.5 Outline of the Thesis and Main Contributions . . . . . . . . . . 14

1.6 List of Publications . . . . . . . . . . . . . . . . . . 18

2 Diversity Techniques 23

2.1 FEC and Interleaving . . . . . . . . . . . . . . . . . 23

2.2 Multiple Antennas . . . . . . . . . . . . . . . . . . . . 30

2.3 Rotated Constellations . . . . . . . . . . . . . . . . 34

2.4 Time Diversity Techniques . . . . . . . . . . . . . . . 35

2.5 Frequency Diversity Techniques . . . . . . . . . . . . . . . . 44

2.6 Space Diversity Techniques _. . . . . . . . . . . . . . . 47

2.7 Conclusions . . . . . . . . . . . . . . 52

3 Information-Theoretic Investigation of Diversity Techniques $\mathbf{5 5}$

3.1 Capacity of Fading Channels . . . . . . . . . . . . . . 56

3.2 Time, Frequency and Space Diversity . . . . . . . . . . . . . . 59

3.3 Rotated Constellations . . . . . . . . . . . . . . . . . 68

3.4 Conclusions . . . . . . . . . . . . . . . 72

4 Combined Diversity in Current Systems $\quad 75$

4.1 Combined Space and Time Diversity in DVB-T . . . . . . . . . 77

4.2 Combined Space and Time Diversity in DVB-T2 . . . . . . . . 88

4.3 Performance Comparison between DVB-T and DVB-T2 . . . . 105

4.4 Conclusions ...................... 106 
5 Combined Diversity in Next Generation Systems 109

5.1 Time Diversity . . . . . . . . . . . . . . . . . . 110

5.2 Frequency Diversity . . . . . . . . . . . . . . . 120

5.3 Space Diversity . . . . . . . . . . . . . . . . . . . . . . 122

5.4 Combined Diversity . . . . . . . . . . . . . . . . 125

5.5 Rotated Constellations . . . . . . . . . . . . . . . . . 126

5.6 Conclusions . . . . . . . . . . . . . . . . . . . 132

6 Design Guidelines and Conclusions 135

6.1 Concluding Remarks . . . . . . . . . . . . . . . . . 135

6.2 Recommendations . . . . . . . . . . . . . . . . . . . . 139

6.3 Future Research Topics . . . . . . . . . . . . . . . . . . . 142

$\begin{array}{ll}\text { References } & 145\end{array}$ 


\section{List of Abbreviations}

AL-FEC Application Layer - Forward Error Correction

ARQ Automatic Repeat Request

ATSC Advanced Television Systems Committee

ATSC-M/H Advanced Television Systems Committee - Mobile/Handheld

AWGN Additive White Gaussian Noise

BB-iFEC BaseBand - inter burst Forward Error Correction

BBFER Base Band Frame Error Ratio

BCH Bose Chadhuri Hocquenghem

BER Bit Error Ratio

BI Block Interleaving

BICM Bit Interleaved Coded Modulation

BICM-ID Bit Interleaved Coded Modulation with Iterative Decoding

BPSK Binary Phase-Shift Keying

bpc bits per cell

bps bits per second

CDD Cyclic Delay Diversity

CDP Content Delivery Protocols

CI Convolutional Interleaving 
CM Coded Modulation

CMMB China Mobile Multimedia Broadcasting

CNR Carrier to Noise Ratio

COFDM Coded Orthogonal Frequency-Division Multiplexing

CRC Cyclic Redundancy Check

CSI Channel State Information

DD Delay Diversity

DMT Diversity-Multiplexing Tradeoff

DTMB Digital Terrestrial Multimedia Broadcast

DTT Digital Terrestrial TV

DVB Digital Video Broadcasting

DVB-H Digital Video Broadcasting - Handheld

DVB-NGH Digital Video Broadcasting - Next Generation Handheld

DVB-SH Digital Video Broadcasting - Satellite services to Handheld devices

DVB-S2 Digital Video Broadcasting - Satellite 2nd Generation

DVB-T Digital Video Broadcasting - Terrestrial

DVB-T2 Digital Video Broadcasting - Terrestrial 2nd Generation

EGC Equal Gain Combining

eSFN enhanced Single Frequency Network

eSM enhanced Spatial Multiplexing

FEC Forward Error Correction

FEF Future Extension Frame

FFT Fast Fourier Transform

FIFO First-In First-Out

FOBTV Future of Broadcast Television 
GC Golden Code

HDPC High Density Parity Check

HDTV High Definition TV

HM Hierarchical Modulation

HP High Priority

ICI Inter-Carrier Interference

IETF Internet Engineering Task Force

IP Internet Protocol

IPDC Internet Protocol Datacast

IPPER Internet Protocol Packet Error Ratio

ISDB-T Integrated Services Digital Broadcasting - Terrestrial

iTEAM Instituto de Telecomunicaciones y Aplicaciones Multimedia

ITS Intermediate Tree Shadowed

IU Interleaving Units

LDPC Low Density Parity Check

LL-FEC Link Layer - Forward Error Correction

LLR Log-Likelihood Ratio

LMS Land Mobile Satellite

LoS Line-of-Sight

LP Low Priority

LT Luby Transform

L1 Layer 1

pdf probability density function

MAP Maximum A Posteriori

MBMS Multimedia Broadcast Multicast Services 
MIMO Multiple-Input Multiple-Output

MISO Multiple-Input Single-Output

ML Maximum Likelihood

MMSE Minimum Mean Square Error

MODCOD Modulation and Coding

MPE-FEC Multi Protocol Encapsulation - Forward Error Correction

MPEG-2 Moving Picture Experts Group 2

MPE-iFEC Multi Protocol Encapsulation - inter burst Forward Error Correction

MRRC Maximal Ratio Receiver Combining

nLoS non Line-of-Sight

OFDM Orthogonal Frequency-Division Multiplexing

PD Phase Diversity

PL-FEC Physical Layer - Forward Error Correction

PLP Physical Layer Pipe

p-t-m point-to-multipoint

p-t-p point-to-point

QAM Quadrature Amplitude Modulation

QoE Quality of Experience

QoS Quality of Service

QPSK Quadrature Phase-Shift Keying

RF Radio Frequency

RS Reed Solomon

RSC Recursive Systematic Convolutional

SC Selection Combining 
SD Subcarrier Diversity

SFBC Space-Frequency Block Code

SFN Single Frequency Networks

SIMO Single-Input Multiple-Output

SISO Single-Input Single-Output

SM Spatial Multiplexing

SSD Signal-Space Diversity

StatMux Statistical Multiplexing

SU Suburban

SVC Scalable Video Coding

TDI Time De-Interleaving

T-DMB Terrestrial - Digital Multimedia Broadcasting

TFS Time-Frequency Slicing

TI Time Interleaving

TS Transport Stream

TSPER Transport Stream Packet Error Ratio

TU6 Typical Urban 6

UL-FEC Upper Layer - Forward Error Correction

UHF Ultra High Frequency

UL Uniform-Late

VBR Variable Bit Rate

XPD Cross Polarization Discrimination

ZF Zero Forcing

2DRC Two-Dimensional Rotated Constellations

3DTV Three-Dimensional TV

3GPP Third Generation Partnership Program

4DRC Four-Dimensional Rotated Constellations 


\section{Chapter 1}

\section{Introduction}

\subsection{Background}

\subsubsection{Evolution of Terrestrial Mobile Broadcasting Sys- tems}

Broadcasting systems are designed for the efficient provision of services (radio, TV) to mass audiences by means of point-to-multipoint (p-t-m) transmissions. Only these systems can deliver a wide range of services without any limitation in the number of users. The utilization of broadcasting systems for the delivery of Digital Terrestrial TV (DTT) has grown very strongly during the last decade, and DTT networks are already in place in many countries all over the world. Today, several broadcasting technologies are available for the provision of DTT services: the Advanced Television Systems Committee (ATSC) in North America, the Integrated Services Digital Broadcasting - Terrestrial (ISDB-T) in Japan, the Terrestrial - Digital Multimedia Broadcasting (T-DMB) in Korea, or the Digital Terrestrial Multimedia Broadcast (DTMB) in China. In addition, the growing interest of mobile TV in the last years has motivated the development of broadcasting systems for the provision of mobile services in handheld devices. This is the case of the Advanced Television Systems Committee - Mobile/Handheld (ATSC-M/H) in North America, the one-segment in Japan, or the China Mobile Multimedia Broadcasting (CMMB) in China. The high fragmentation of terrestrial broadcasting systems around the world, and the necessity to benefit from economies of scale, has motivated endeavours to promote the cooperation among different networks and transmission systems. Launched in 2012, the Future of Broadcast Television (FOBTV) initiative aims to create a common framework for the next generation of terrestrial 


\section{CHAPTER 1. INTRODUCTION}

broadcasting systems. This includes the definition of user cases, scenarios and requirements, as well as recommendations on major technologies to be used as the basis for new standards.

This dissertation centers on the European family of standards developed by the Digital Video Broadcasting (DVB) consortium ${ }^{1}$. The first generation European standard for the provision of DTT services was published by the DVB project in 1998 and is known as Digital Video Broadcasting - Terrestrial (DVB-T) [1]. Since then, DVB-T has become the most widely adopted DTT system in the world, with nearly 200 million receivers sold in more than 40 countries [2]. Despite the tremendous success of DVB-T, the recent technological advances in communications and the appearance of new digital content such as High Definition TV (HDTV) or Three-Dimensional TV (3DTV) [3], motivated the development of a second generation DTT system. In 2008, the DVB project published the Digital Video Broadcasting - Terrestrial 2nd Generation (DVB-T2) standard [4], which provides a $50 \%$ increase of spectral efficiency over its predecessor [5]. DVB-T2 trials have taken place in many European countries, and commercial DVB-T2 services are already in the air in UK, Italy, Sweden and Finland. In order to address the provision of mobile services to handheld devices, the DVB project published the standard known as Digital Video Broadcasting - Handheld (DVB-H) [6] in 2004, and later in 2007, the standard known as Digital Video Broadcasting - Satellite services to Handheld devices (DVB-SH) [7].

The adoption of mobile TV services has not fulfilled the initial expectations due to the lack of a successful business model and the high costs associated to the deployment of new mobile broadcasting networks [8]. Mobile channels are significantly more challenging than traditional fixed scenarios due to the user mobility, the utilization of more compact antennas, and the reception at ground level. In order to provide good coverage levels for mobile broadcasting services, it is necessary to deploy dense networks with a large number of sites. This penalty is particularly evident for high coverage targets (e.g. over $90 \%$ of service area locations) [9]. As a result, the costs associated to the deployment of mobile broadcasting networks with good coverage are only justificable in urban areas with a high user density, where it is possible to eventually recover the initial investment in infrastructure. In order to avoid this situation, it would be desirable to perform an incremental deployment that follows the user demand [10]. Within this scenario, the cellular network would prevent the over-dimensioning of the broadcasting capacity, and would ensure the service continuity in low populated areas with no initial broadcasting infrastructure.

\footnotetext{
${ }^{1}$ Instituto de Telecomunicaciones y Aplicaciones Multimedia (iTEAM) is a full member of the DVB standardization forum, and is actively participating in the standardization process of next generation terrestrial mobile broadcasting systems.
} 
However, even if this hybrid approach is very promising from a mere technical point of view, it is problematic as it requires the cooperation between the broadcasting and the cellular players.

In this context, the utilization of DTT networks for the simultaneous delivery of fixed and mobile services can facilitate the introduction of mobile broadcasting services by reusing the same content, spectrum, and infrastructure. Despite the fact that DVB-T was originally designed for fixed and portable reception, the widely availability of deployed DVB-T networks has motivated the provision of vehicular DVB-T services in several countries such as Germany or Taiwan [11]. In a similar manner, the good performance of DVB-T2 in mobile scenarios has motivated several trials and studies regarding the provision of mobile services in DVB-T2 networks. DVB-T2 supports a per-service configuration of transmission parameters, including modulation, coding and interleaving. This allows for the provision of services targeting different user cases, i.e. fixed, portable and mobile, in the same frequency channel. In addition, a new profile known as T2-Lite has been recently included in the DVB-T2 standard to reduce the complexity of receivers, and to improve the coexistence of fixed and mobile services. The new profile makes possible to efficiently share the capacity of one frequency channel in a time division manner between DVB-T2 and T2-Lite services. The DVB project is also standardizing the next generation broadcasting system for the provision of mobile services to handheld devices known as Digital Video Broadcasting - Next Generation Handheld (DVB-NGH) [12]. DVB-NGH is expected to outperform in both coverage and capacity existing mobile broadcasting standards such as DVB-H and DVB-SH. As with T2-Lite, the new standard is being designed so that DVB-NGH and DVB-T2 services can coexist in the same frequency channels.

\subsubsection{The Challenges of the Mobile Broadcasting Channel}

The lack of feedback information in mobile broadcasting systems prevents the utilization of Automatic Repeat Request (ARQ) or link adaptation techniques, typically used in point-to-point (p-t-p) transmissions (e.g. cellular systems) to recover from transmission errors. As a result, broadcasting systems have to rely on Forward Error Correction (FEC) to ensure error-free communication in noisy channels [13]. FEC mechanisms add redundant data before transmission to protect the information from channel impairments. Shannon theorem states that by means of proper encoding and decoding techniques, it is possible to achieve error-free communication as long as the information rate does not 


\section{CHAPTER 1. INTRODUCTION}

exceed the capacity of the channel ${ }^{2}$ [14]. The FEC scheme included in the first generation of broadcasting systems, which is based on the concatenation of convolutional and Reed Solomon (RS) codes, achieves a performance that is several dBs away from the channel capacity [15]. Nowadays, Low Density Parity Check (LDPC) codes and turbo codes allow wireless communication systems to be within $1 \mathrm{~dB}$ of the channel capacity in Additive White Gaussian Noise (AWGN) [16, 17]. The second generation of DVB systems already incorporate LDPC codes for channel coding [18], and hence, major gains cannot be expected by means of better FEC codes.

Mobile channels are not affected only by thermal noise but also by fluctuations of the received signal strength known as fading [19, 20]. The fades encountered in the received signal represent a very significant degradation of the quality of communication in mobile channels, since receivers experiences a loss of information whenever the mobile channel suffers a deep fade. The fades encountered in mobile channels are localized in time, frequency and space, meaning that a sufficient change in the transmission instant of time, the transmitted frequency, or the receiver location results in an independent fading realization. This is referred to as channel selectivity.

In the time domain, mobile reception is characterized by fast and slow variations of the received signal strength, known as fast fading and shadowing respectively. Fast fading is caused by the constructive and destructive interference of multiple waves along the user trajectory, whereas shadowing arises due to the varying obstruction of the line of sight between the transmitter and the receiver. On the other hand, frequency selective fading is caused by the arrival of long delayed echoes (typically known as taps) of the transmitted signal. Orthogonal Frequency-Division Multiplexing (OFDM) provides good protection against multipath propagation by dividing the spectrum in multiple orthogonal subcarriers so that each subcarrier experiments a flat-frequency (i.e. non frequency selective) channel [21]. The presence of frequency selectivity in OFDM systems results in signal strength variation across the subcarriers that constitute the OFDM symbols. DVB-T was the first commercial wireless system to incorporate OFDM and since then, it has been widely used in wireless communications [22], including broadcasting and cellular systems. In addition to the frequency selectivity caused by multipath propagation, large differences in the received signal strength can be found between different Radio Frequency (RF) channels due to the frequency dependency of the transmitters, the receivers, and the propagation environment [23]. In this context, the reli-

\footnotetext{
${ }^{2}$ The capacity of the AWGN channel in bits per second (bps) is given by $C_{\text {AWGN }}=W \log _{2}(1+S)$, where $W$ is the signal bandwidth and $S$ is the signal to noise power ratio.
} 
ability of the information against time and frequency selective fading can be increased by means of diversity in the time, frequency and space domains.

\subsubsection{Closing the Gap between Fading Channels and AWGN}

The principle of diversity is to provide the receiver with multiple versions of the transmitted signal, where each version is received through a distinct channel. The number of channels is usually known as the number of diversity branches [19]. In order to obtain a high degree of improvement from diversity, the fading of the different branches should have a low cross-correlation so that the chance of a deep fade occurring in all the branches simultaneously is reduced. Moreover, the mean power available from each branch should be almost equal. Otherwise, the signal at the weaker branch may not be useful even if it is less faded than the other branches. If the information is transmitted over $d$ diversity branches with independent fading and similar mean power, it is possible to reduce the error probability as a function of the number of branches. In particular, the error probability in fading channels decays proportionally to $1 / \mathrm{SNR}^{\mathrm{d}}$, where $d$ is referred to as the diversity order of the system.

Time and frequency diversity in mobile broadcasting systems is generally achieved by means of FEC and interleaving. Each codeword is partitioned in multiple fragments that are transmitted in different channel realizations. This way, the FEC code exploits the diversity of the system by averaging the fading in each codeword. Due to the correlation encountered in the mobile channel, it is necessary to spread the codewords over time and frequency by means of interleaving so that a low correlation is achieved within the codewords. Space diversity, on the other hand, is achieved by means of multiple antennas at the transmitter and/or at the receiver side. The signals from different antenna paths are combined in the receiver to average the fading between antennas. It should be pointed out that there is a diminishing marginal return of the diversity gain that is achieved in each domain [24]. In other words, the higher the diversity gain, the lower the benefits that are obtained with additional diversity. Fig. 1.1 illustrates the combined use of diversity in the time, frequency and space domains. Here, the channel encountered across four antenna paths is first averaged at the receiver in order to exploit the diversity in the space domain. The resulting signal is then passed to the FEC decoder, which exploits the diversity in the time and frequency domains when decoding the codewords.

While the combination of FEC and interleaving relies on the error-correcting capabilities of the code to achieve diversity, it is also possible to embed some degree of diversity into the constellation symbols by means of Signal-Space Diversity (SSD) [25]. The main idea behind SSD is to apply a certain rotation 


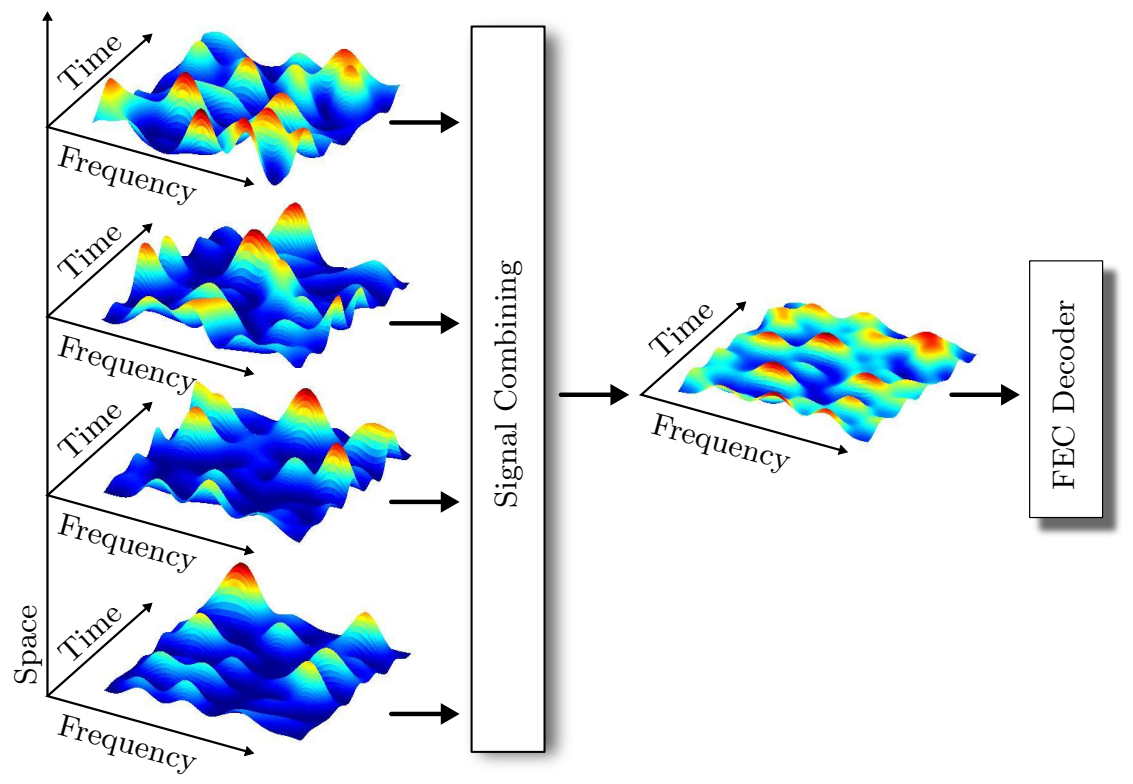

Figure 1.1: Combined diversity in time, frequency and space.

to the constellation so that any two symbols present the maximum number of distinct components. After this, each component is transmitted through a different channel realization in the time, frequency, or space domain. The utilization of SSD improves the robustness against fading without the need of additional parity data, but requires extra complexity for demodulating all the symbol components in a joint manner.

\section{Time Diversity}

The main idea of Time Interleaving (TI) is to interlace multiple codewords so that each codeword is transmitted over an extended period of time [26]. The total period of time over which one codeword is transmitted is referred to as interleaving duration. Generally speaking, interleaving durations in the order of hundreds of milliseconds are sufficient to combat fast fading in the Ultra High Frequency (UHF) band, whereas several seconds are needed in the case of shadowing. The implementation of TI has an important impact on the hardware complexity of receivers. In order to perform time deinterleaving, receivers must incorporate enough memory to store all the codewords that were interleaved together at the transmitter. As a result, the maximum interleaving duration that can be provided for any given data rate is constrained by the 
Time De-Interleaving (TDI) memory that is available in the receivers. The implementation of TI and FEC at the physical or upper layers has a great impact on the system performance and on the TDI memory requirements. Physical Layer - Forward Error Correction (PL-FEC) generally provides better correction capabilities, whereas Upper Layer - Forward Error Correction (UL-FEC) allows the provision of long TI with more relaxed hardware requirements [27].

The utilization of TI also increases the end-to-end latency and specially the zapping time. While the end-to-end latency is not critical for the majority of services, the zapping time plays an important role in the user experience, and is considered as a crucial parameter for TV usability. Generally, it is considered that zapping times longer than $2 \mathrm{~s}$ are felt as annoying, whereas less than $500 \mathrm{~ms}$ are seen as instantaneous [28]. Some interleaving schemes allow the utilization of fast zapping and parity recovery techniques, which are capable of reducing the zapping time down to tolerable values even when interleaving duration of several seconds are used. In addition to TI, mobile broadcasting systems generally employ discontinuous transmissions in order to achieve power saving and reduce the power consumption of receivers. This is known as timeslicing. By switching-off their front ends in the gaps of time between bursts of information, receivers can reduce the overall power consumption. However, this reduces the robustness of the transmitted signal against fading, since the maximum time diversity is achieved when no time slicing is used (i.e. maximum power consumption) and the codewords are continuously transmitted over time.

\section{Frequency Diversity}

If FEC is used together with OFDM, the codewords can be distributed across multiple subcarriers in order to achieve frequency diversity. The combined utilization of FEC and OFDM is usually referred to as Coded Orthogonal Frequency-Division Multiplexing (COFDM). By means of COFDM, it is possible to retrieve the information from subcarriers that have been erased by frequency selective fading. In order to increase the diversity achieved in the frequency domain, frequency interleaving can be used in COFDM systems to spread the codewords across the entire signal bandwidth. Due to the fact that frequency interleaving is performed in the frequency direction, it has no constraints in terms of latency or channel switching time. While the signal bandwidth is generally limited to one RF channel, it may be possible to extend the frequency interleaving across multiple channels by means of Time-Frequency Slicing (TFS). TFS employs a combination of frequency hopping and time 


\section{CHAPTER 1. INTRODUCTION}

slicing to achieve better frequency diversity, and also to increase the system capacity by means of more efficient Statistical Multiplexing (StatMux) ${ }^{3}$.

\section{Space Diversity}

The utilization of multiple transmit and/or receive antennas has become a key technology in the last years for improving the performance of wireless communications systems [29]. If multiple antennas are used, and the distance between antennas is large enough, the channel between different antenna pairs fade independently, and space diversity can be extracted by averaging the fading across the independent signal paths. While co-polarized antennas can obtain important diversity gains in the case of vehicular reception, they are generally impractical for handset-based reception in the UHF band, as the required separation between antennas is far beyond the dimensions of typical handsets (e.g. $15 \mathrm{~cm}$ at $600 \mathrm{MHz}$ ). In contrast, multiple antenna systems based on polarization diversity rely on the low degree of correlation between polarization paths, and are better suited to handset-based reception [30].

Compared to the utilization of one antenna at the transmitter and the receiver side or Single-Input Single-Output (SISO), the utilization of multiple receive antennas is usually known as receive diversity or Single-Input MultipleOutput (SIMO). SIMO systems provide a diversity gain due to the averaging of fading across the different antenna paths, and also an array gain due to the coherent combination of signals at the receiver side. In contrast with SIMO, the utilization of multiple antennas at the transmitter side is referred to as transmit diversity or Multiple-Input Single-Output (MISO). A Space-Frequency Block Code (SFBC) operates with the information symbols of adjacent subcarriers across the transmit antennas, so that they can be combined in reception in an optimum way. A well-known SFBC is the Alamouti code [31], which can achieve full diversity with low (linear) decoding complexity. The utilization of multiple antennas at both ends is referred to as Multiple-Input MultipleOutput (MIMO). In addition to diversity and array gains, MIMO systems may achieve a multiplexing gain by increasing the transmission rate of information symbols [32]. A traditional method of exploiting the multiplexing capabilities of the MIMO channel is known as spatial multiplexing, and consists in multiplexing different data streams across the transmit antennas.

Generally speaking, the utilization of MIMO requires the implementation of more complex signal processing at the receiver side for the decoding of multiple

\footnotetext{
${ }^{3}$ TFS was initially proposed in DVB-T2 but was finally included only as an informative annex due to the need of implementing more than one receiver chain. The low bit rates of mobile TV services allow the operation of TFS with single tuner receivers, and the technique has been adopted in DVB-NGH.
} 
signals, and also an increase in the number of pilots that must be transmitted for channel estimation purposes ${ }^{4}$. Some techniques enable the use of multiple transmit antennas for better diversity without the need of additional pilots or specific signal processing at the receive side. The main aim of these techniques is to create a single transmit antenna channel with a higher frequency diversity that is exploited by the FEC code. This is the case of Delay Diversity (DD), Cyclic Delay Diversity (CDD), Phase Diversity (PD) and Subcarrier Diversity (SD) $[33,34]$. Although the utilization of these techniques does not result in an optimal averaging of fading, they achieve a higher diversity than single transmit antenna configurations.

\subsection{Problem Formulation}

The provision of mobile broadcasting services is challenged by the severe propagation conditions of the mobile channel. Since the latest generation of mobile broadcasting systems already incorporate capacity-achieving codes such as LDPC or turbo codes, major further gains cannot be achieved with better FEC codes. In this context, the combined utilization of time, frequency and space diversity constitutes a key instrument to reduce the costs associated to the deployment of mobile broadcasting networks while providing the necessary capacity for the delivery of new services.

Mobile broadcasting systems currently encompass time diversity across hundreds of milliseconds, frequency diversity inside a single frequency channel, and space diversity with multiple antennas at the receiver side. So far, DVB-SH is the only mobile broadcasting system that exploits time diversity across several seconds by means of long TI, and no system exploits the diversity in the frequency domain across several RF channels, or in the space domain with multiple antennas at the transmitter and the receiver side. The next generation mobile broadcasting standard DVB-NGH will be the first system to incorporate the use of diversity in the three domains by means of long TI, TFS, and MIMO.

The utilization of diversity in the three domains: time, frequency and space, cannot be studied in a separate manner for several reason. In the first place, the total gain that can be achieved by means of diversity in the three domains is limited by the diminishing marginal returns of diversity, and hence, the gain of combining different diversity techniques may be lower than the sum

\footnotetext{
${ }^{4}$ OFDM systems generally employ pilot-based channel estimation. The channel state is estimated in the pilot subcarriers and afterwards is interpolated in frequency and/or time across the rest of subcarriers and/or OFDM symbols. The utilization of pilots decreases the amount of subcarriers that can be used for information, and thus, it reduces the overall system capacity.
} 
of the individual gains. In the second place, each type of diversity entails a different trade-off regarding very important aspects such as latency, power consumption, and hardware complexity. As a result, the use of time, frequency and space diversity must be configured in a joint manner in order to optimize the system performance not only in terms of robustness, but also in terms of latency, zapping time, power saving, and hardware complexity. For example, by means of extensive frequency and space diversity it might be possible to reduce the requirements for TI, allowing services to be delivered with a lower latency and shorter zapping times. Similarly, large amounts of time and frequency diversity may diminish the gain of MIMO techniques, sparing the costs of multiple transmit antennas.

\subsection{Objectives and Thesis Scope}

The main research topic of the dissertation is the combined use of diversity in the time, frequency, and space domains for the provision of mobile services in terrestrial broadcasting systems. In a first approach, the performance is evaluated from an information-theoretic point of view. The goal is to compute the gains that can be achieved with diversity techniques in mobile scenarios regardless of the particular system in which they are implemented. Since the results are based on the outage channel capacity, they represent the ultimate performance of mobile broadcasting systems, and can be used to compare the performance of real systems with the theoretical maximum.

For the evaluation of diversity in real systems, the dissertation focuses on the DVB family of standards, although the investigations are of generic nature and can be applied to future evolutions of standards such as the Japanese ISDB-T or the American ATSC. In addition to pure mobile broadcasting systems like DVB-H and DVB-NGH, terrestrial broadcasting systems aimed at stationary and portable reception such as DVB-T and DVB-T2 are also within the scope of this thesis. The convergence of the fixed and the mobile paradigms can reduce the initial investment that is required for the start-up of commercial mobile services by reusing content, spectrum, and infrastructure. In this case, the objective is to evaluate the suitability of terrestrial broadcasting networks for the simultaneous provision of terrestrial and mobile services, and how the utilization of diversity techniques can improve it.

The combined use of diversity in the time, frequency and space domain has been carried out under the standardization process of DVB-NGH, the first mobile broadcasting system to exploit all three types of diversity by incorporating at the physical layer long TI, TFS, and MIMO. During the standardization process of DVB-NGH, these techniques were studied in a separate manner, and 
therefore, a joint investigation such as the one developed in this dissertation is novel.

Finally, the results and conclusions obtained in this dissertation are materialized in the form of recommendations for the use of diversity techniques in existing and future terrestrial broadcasting systems.

\subsection{Related Work}

This section provides a short overview of the prior related work regarding the use of time, frequency and space diversity in mobile broadcasting systems.

\subsubsection{Time Diversity in Mobile Broadcasting Systems}

Although DVB-T was not originally envisioned for the provision of mobile services, its performance in mobile scenarios has been extensively evaluated by means of simulations, laboratory measurements and field trials [35, 36]. These investigations demonstrate that, while DVB-T was designed for fixed and portable reception, some of its more robust modes allow the reception of mobile services as well. Results show that with proper channel estimation techniques, the main limiting factor for the provision of DVB-T services in mobile channels is not the Inter-Carrier Interference (ICI) caused by the Doppler spread, but rather the lack of TI.

DVB-H reutilizes the physical layer of DVB-T and incorporates Multi Protocol Encapsulation - Forward Error Correction (MPE-FEC) at the link layer to improve the system performance in mobile scenarios [37]. In particular, MPE-FEC is an UL-FEC mechanism that provides interleaving durations in the order of 100-200 ms by means of a block interleaver and a RS code. According to field measurements performed in a wide range of reception conditions [38], the gain of MPE-FEC depends on the user velocity, being largest in vehicular scenarios with speeds higher than $70 \mathrm{~km} / \mathrm{h}$.

As with DVB-T, the performance of DVB-T2 in mobile scenarios has brought attention in the literature [39]. In particular, the robustness of the signalling data in mobile channels has been studied in several works [40, 41]. Although DVB-T2 incorporates a time interleaver at the physical layer for the application data, the interleaving duration of the signalling data is limited to a few OFDM symbols. Simulation results have shown that for certain configurations, the robustness of the signalling data in mobile scenarios may not be sufficiently high as compared with the application data. Nevertheless, it should be pointed out that these results were obtained under the assumption of Genie 
aided demapping [42], which may not represent the true performance of real receivers ${ }^{5}$.

The performance of long TI has been investigated in the context of DVB-SH. DVB-SH incorporates long TI to recover from the long signal outages caused by obstacles such as buildings or trees in Land Mobile Satellite (LMS) scenarios. At the physical layer, the standard incorporates a convolutional interleaver that can provide interleaving durations up to several seconds (i.e. $10 \mathrm{~s}$ ) with fast zapping and parity recovery. At the link layer, an UL-FEC technique known as Multi Protocol Encapsulation - inter burst Forward Error Correction (MPE-iFEC) is included for extending the protection across multiple timeslicing bursts [43]. It is based on an sliding window interleaver and the same RS code as MPE-FEC. The results obtained in field tests [44, 45] have revealed that the use of TI at the physical layer outperforms MPE-iFEC in every reception scenario. Although long TI achieves the largest gain in LMS channels, it has been shown to achieve significant gains in terrestrial channels as well $[10,46,47]$. In particular, the utilization of Application Layer - Forward Error Correction (AL-FEC) has been proposed for extending the protection of DVB-H services across multiple time-slicing bursts. AL-FEC was standardized in DVB-H for file delivery services [48], and is based on Raptor codes [49] for encoding large amounts of information in a joint manner. Although AL-FEC is currently not standardized for the protection of streaming services, it can be implemented in DVB-H with some minor modifications [46].

\subsubsection{Frequency Diversity in Mobile Broadcasting Systems}

The investigations performed in the context of mobile DVB-T reception $[35,36]$ have confirmed that the combination of COFDM and frequency interleaving generally achieves sufficient frequency diversity in the terrestrial $8 \mathrm{MHz}$ channels of the UHF band. Nevertheless, recent studies have shown the potential benefits of extending the frequency interleaving across multiple RF channels by means of TFS [50]. Field measurements performed by Teracom, the Swedish terrestrial broadcast network operator, pointed out that the received signal strength depends significantly on the relative position of the receiver with the transmitter antenna pattern in the horizontal plane [23]. According to the measurements, the potential gain of TFS across four frequency channels can be higher than $4.5 \mathrm{~dB}$ in fixed reception scenarios [51]. However, no investi-

\footnotetext{
${ }^{5}$ Genie aided demapping is considered to be representative of the ultimate performance of iterative decoding and demapping techniques. However, the utilization of such techniques requires a significant increase in terms of hardware complexity, and at the present time, such iterative techniques are not being implemented in real receivers.
} 
gation has been performed in mobile reception conditions, and it is uncertain how much of this gain can be transfered to the mobile scenario.

\subsubsection{Space Diversity in Mobile Broadcasting Systems}

In order to achieve space diversity, the utilization of SIMO by means of copolarized antennas has been traditionally proposed in DVB-T and DVB-H for vehicular reception $[52,53]$. Laboratory measurements and field tests have shown that the use of multiple receive antennas lowers the Carrier to Noise Ratio (CNR) that is required for reception and, at the same time, increases the maximum speed that is supported due to better protection against Doppler. The results also reveal that the improvement achieved by SIMO is significant even in the presence of strong correlation between antennas [36].

Although it was not included in the standard, the utilization of MISO has been proposed in DVB-T and DVB-H for improving the reception in mobile scenarios. Since SFBCs like the Alamouti code cannot be used in real DVB-T systems because of the lack of dedicated pilot patterns and signal processing at the receiver side, techniques such as CDD and DD have been considered instead $[34,54,55]$. Simulation and laboratory measurements have shown that although the gain is lower than with SIMO, both CDD and DD can improve the reception of DVB-T services in mobile scenarios. As with SIMO, the use of CDD and DD can still improve the system performance when some degree of correlation exists between the antennas [56].

The distributed MISO technique included in DVB-T2 has been also the subject of several studies $[57,58]$. Simulation results with two transmitters show that the improvement brought by this technique is highly dependent on the power imbalance that exists between the signals from different transmitters [57]. If the power imbalance is high enough, the use of distributed MISO with suboptimal decoders might degrade the system performance compared to non-MISO. Simulations with three and four transmitters show that the gain of distributed MISO is also conditioned by the network configuration [58], i.e. number of transmitters and separation between them.

In addition to SIMO and MISO, full MIMO has been investigated in the context of DVB-T2 [59, 60]. Laboratory and field measurements have shown that the use of multiple antennas at both ends has the potential to provide gains up to $75 \%$ in terms of capacity. The results also reveal that, compared to traditional co-polar antennas, the use of cross-polarized antennas can provide higher gains in Line-of-Sight (LoS) scenarios, or in the presence of strong correlation between antennas [60]. 


\subsection{Outline of the Thesis and Main Contributions}

The thesis is organized in six main chapters as follows: Chapter 2 describes the utilization of diversity in mobile broadcasting DVB systems. The fundamentals of FEC and interleaving, which are used to achieve time and frequency diversity, and the main concepts of MIMO, which is used to achieve array, diversity and multiplexing gains in the space domain, are described in detail. Chapter 2 also describes the trade-offs associated to each type of diversity, and reviews the mechanisms that are included in mobile broadcasting systems. Chapter 3 evaluates the use of diversity from an information-theoretic point of view. The potential gain of diversity techniques, including long TI, TFS, and MIMO, is computed according to the outage channel capacity. Chapter 4 investigates the utilization of diversity techniques for the provision of mobile services in DVB-T, DVB-H, and DVB-T2 networks. In addition to simulations, the chapter presents a discussion regarding the advantages of diversity techniques in terms of network planning. Chapter 5 is dedicated to next generation systems. The combined utilization of diversity in the three domains by means of long TI, TFS, and MIMO, is investigated in the context of DVB-NGH. This investigation includes BaseBand - inter burst Forward Error Correction (BB-iFEC) and rotated constellations. The first technique was proposed in the standardization process for long TI with lower zapping times. The second technique has been included in the standard to improve the reception robustness against fading by means of SSD. Finally, Chapter 6 summarizes the results obtained in previous chapters and presents a number of recommendations for the use of diversity techniques in mobile broadcasting systems. Suggestions on future research topics are also provided.

The key contributions of the dissertation in each chapter are summarized next. Notice that although Chapter 2 presents no original contribution, it lays the foundations for the investigation of diversity techniques in mobile broadcasting systems. The reader should refer to Section 1.6 for a complete list of publications originated from the work carried out in the dissertation.

\section{Chapter 3}

The main contribution of this chapter is the computation, based on the outage channel capacity, of the diversity gains that can be achieved in mobile broadcasting systems. Although the methodology followed in this chapter is well known [32, 61], its utilization for a joint investigation of space, time, and frequency diversity in mobile broadcasting systems is novel. The mutual influence of each type of diversity, and the potential gains that can be achieved 
by combining the three types of diversity, are studied in this theoretical framework. Furthermore, the performance of rotated constellations is investigated by evaluating the information-theoretic capacity of Bit Interleaved Coded Modulation (BICM).

\section{Chapter 4}

This chapter focuses on the combined use of time and space diversity for the provision of mobile services in the first and second generation of terrestrial broadcasting DVB systems. This includes DVB-T, DVB-H, and DVB-T2. In order to achieve time diversity in DVB-T and DVB-H systems, we consider the utilization of UL-FEC techniques due to the lack of TI at the physical layer. For space diversity, the investigation is restricted to SIMO due to compatibility issues with existing networks and systems. The work performed by the author in this topic lead to a patent application and the development of a prototype based on AL-FEC for the protection of DVB-T services in mobile scenarios [8]. The prototype is fully compatible with legacy DVB-T receivers and allows the decoding of AL-FEC protected services to be done in generic software processors, even on low complexity receivers.

In the case of DVB-T2, we analyze the use of TI at the physical layer and the trade-offs that are possible in terms of time diversity, data rate, latency, and power saving. The thorough investigation of TI that is presented in this chapter has culminated in a contribution to the implementation guidelines of DVB-T2 [42]. In addition, we consider the utilization of AL-FEC to overcome the limitations of the physical layer and achieve long TI with lower zapping times. The investigation of TI also takes into account the reduced time diversity of the signalling information, and evaluates the utilization of advanced decoding techniques for better robustness in mobile scenarios.

In order to achieve space diversity in DVB-T2, we have investigated the use of SIMO and the distributed MISO technique included in the standard. Distributed MISO techniques are very interesting in the context of terrestrial broadcasting networks, as they can be implemented without additional costs for network operators. So far, the performance of these techniques has been studied in LoS reception conditions [57] for stationary and portable reception. In this chapter, we show that the gain of distributed MISO in the case of mobile channels is much more limited depending on the power imbalance and the delay between the signals of different transmitters.

The provision of mobile services in DVB-T and DVB-T2 systems is also analyzed from a network planning point of view. The gains of diversity techniques are translated into a coverage extension of mobile services, or alternatively, into a reduction of the network infrastructure. We also perform an investigation in 
terms of link budget to obtain the coverage levels of mobile services in networks planned for fixed and portable indoor reception.

\section{Chapter 5}

The work presented in this chapter has been carried out under the standardization process of DVB-NGH, and studies the utilization of time, frequency, and space diversity by means of long TI, TFS, and MIMO. Our investigations show that the solution adopted in the standard for long TI is cumbersome due to the bad performance of LDPC codes against heavy puncturing (erasures). It turns out that in this case, a split FEC approach like BB-iFEC can provide a good compromise between overall robustness and zapping time, as well as more efficient memory usage and improved compatibility with simpler terminals.

Regarding the use of TFS in mobile broadcasting systems, some preliminary studies have been carried out for stationary reception [50], however, it is not certain how much of this gain can be transferred into the mobile scenario. The work presented in this chapter constitutes one of the first investigations regarding the gains of TFS as a result of better frequency diversity in mobile channels.

The investigation on MIMO techniques focuses on two important aspects in mobile broadcasting systems: the amount of capacity that is required for channel estimation, and the utilization of powerful FEC codes. Traditionally, the performance of MIMO techniques is evaluated in terms of uncoded Bit Error Ratio (BER) (i.e. before any FEC code), and assuming that channel estimation at the receiver side is accomplished with no impact in the system capacity. As it is shown in this chapter, very simple techniques can outperform more complex codes with a-priori better properties if the capacity loss due to the transmission of pilot information and the error-correcting capabilities of FEC code are taken into account.

Finally, the chapter investigates the performance of the rotated constellations that have been adopted in DVB-NGH. The additional protection against fading and erasures given by rotated constellation is very interesting for long TI and TFS. In particular, the combination of rotated constellations with an optimized component interleaver can increase the diversity gains in the time and frequency domains as well as reduce the zapping time with long TI.

\section{Chapter 6}

The conclusions and recommendations found in this chapter represent a very useful guideline for the configuration of terrestrial broadcasting systems like DVB-T or DVB-T2, and also for the design of next generation broadcast- 
ing systems such as a possible future evolution of the European DVB-T2, the Japanese ISDB-T, the American ATSC, the Korean T-DMB, or the Chinese CMMB. 


\subsection{List of Publications}

\section{International Journals}

[IJ1] D. Gómez-Barquero, D. Gozálvez, and N. Cardona, "Application layer FEC for mobile TV delivery in IP datacast over DVB-H systems," IEEE Transactions on Broadcasting, vol. 55, no. 2, pp. 396-406, 2009.

[IJ2] D. Gozálvez, D. Gómez-Barquero, T. Stockhammer, and M. Luby, "AL-FEC for improved mobile reception of MPEG-2 DVB-T transport streams," International Journal of Digital Multimedia Broadcasting - special issue on Convergence of Digital TV Systems and Services, 2009.

[IJ3] D. Gómez-Barquero, D. Gozálvez, P. F. Gómez, and N. Cardona, "Fading margin reduction due to interburst upper layer FEC in terrestrial mobile broadcast systems," IEEE Transactions on Vehicular Technology, vol. 60, no. 7, pp. 3110-3117, 2011.

[IJ4] D. Gozálvez, D. Gómez-Barquero, D. E. Vargas, and N. Cardona, "Time diversity in mobile DVB-T2 systems," IEEE Transactions on Broadcasting, vol. 57, no. 3, pp. 617-628, 2011.

[IJ5] D. Gómez-Barquero, P. F. Gómez, D. Gozálvez, B. Sayadi, and L. Roullet, "A novel physical layer split FEC scheme for long time interleaving with fast zapping support," accepted for publication on IEEE Transactions on Broadcasting.

[IJ6] D. Gozálvez, J. López-Sánchez, D. Gómez-Barquero, J. J. Giménez and N. Cardona, "Combined time and space diversity for mobile reception in DVB-T and DVB-T2 systems," accepted for publication on IEEE Vehicular Technology Magazine.

[IJ7] J. López-Sánchez, D. Gómez-Barquero, D. Gozálvez and N. Cardona, "On the provisioning of mobile digital terrestrial TV services to vehicles with DVB-T," accepted for publication on IEEE Transactions on Broadcasting.

[IJ8] J. J. Giménez, D. Gozálvez, D. Gómez-Barquero, and N. Cardona, "A statistical model of the signal strength imbalance between RF channels in a DTT network," accepted for publication on Electronics Letters.

\section{National Journals}

[NJ1] D. Gómez-Barquero et al., "End-to-end digital video broadcasting testbed," Waves, year 2, pp. 134-141, 2010. 


\section{Book Chapters}

[S1] D. Gozálvez, D. Gómez-Barquero, I. Eizmendi, G. Berjón and M. Vélez, "Chapter 6: DVB-T2 for mobile and mobile DVB-T2 (T2-Lite)," Next Generation Mobile Multimedia Broadcasting, CRC Press, to be published in 2013 .

[S2] P. F. Gómez, D. Gómez-Barquero, D. Gozálvez, A. Aniorga and M. Breiling, "Chapter 12: Time Interleaving in DVB-NGH," Next Generation Mobile Multimedia Broadcasting, CRC Press, to be published in 2013.

\section{Patents}

[P1] T. Stockhammer, D. Gómez-Barquero, and D. Gozálvez, Mobile Reception of Digital Video Broadcasting - Terrestrial Services, Application number: 12/714268 Publication number: US 2010/0223533 A1

\section{International Conferences}

[IC1] D. Gozálvez, D. Gómez-Barquero, and N. Cardona, "Performance evaluation of the MPE-iFEC sliding RS encoding for DVB-H streaming services," in IEEE Personal, Indoor and Mobile Radio Conference (PIMRC' 08), Cannes, France, $2008^{6}$.

[IC2] D. Gozálvez, D. Gómez-Barquero, and T. Stockhammer, "Mobile reception of DVB-T services by means of AL-FEC protection," in IEEE International Symposium on Broadband Multimedia Systems and Broadcasting (BMSB'09), Bilbao, Spain, 20096.

[IC3] D. Gozálvez, D. E. Vargas, D. Gómez-Barquero, and N. Cardona, "Performance evaluation of DVB-T2 time interleaving in mobile environments," in IEEE Vehicular Technology Conference (VTC'10 Fall), Ottawa, Canada, $2010^{6}$.

[IC4] D. Gozálvez, V. Pauli, S. Moon, and W. Ko, "Transmit diversity based on subcarrier diversity for OFDM broadcasting systems," in IEEE International Symposium on Broadband Multimedia Systems and Broadcasting (BMSB'11), Erlangen, Germany, 2011.

\footnotetext{
${ }^{6}$ This paper was also presented in the European cooperation network for scientific and technical research COST2100 (www.cost2100.org)
} 
[IC5] D. Gómez-Barquero, P. F. Gómez, D. Gozálvez, B. Sadayi, and L. Roullet, "Base band inter-frame FEC (BB-iFEC) for next generation handheld DVB-NGH," in IEEE International Symposium on Broadband Multimedia Systems and Broadcasting (BMSB'11), Erlangen, Germany, 2011.

[IC6] S. Moon et al., "Enhanced Spatial Multiplexing for Rate-2 MIMO of DVB-NGH System," in IEEE International Conference on Telecommunications (ICT'12), Jounieh, Lebanon, 2012.

\section{National Conferences}

[NC1] D. Gozálvez, D. Gómez-Barquero, and N. Cardona, "Transmisión de servicios de televisión digital móvil en redes DVB-SH," in XVII Jornadas Telecom $I+D$, Valencia, Spain, 2007.

[NC2] D. Gozálvez, D. Gómez-Barquero, F. Camaro, and N. Cardona, "Codificación Reed-Solomon de ventana deslizante para la transmisión de servicios de streaming en redes DVB-SH," in XVIII Jornadas Telecom $I+D$, Bilbao, Spain, 2008.

[NC3] D. Gómez-Barquero, D. Gozálvez, P. F. Gómez, and N. Cardona, "Desarrollo y aplicaciones de un simulador dinámico de TDT móvil DVB-H," in XVIII Jornadas Telecom I+D, Bilbao, Spain, 2008.

[NC4] D. Gozálvez, D. Gómez-Barquero, N. Cardona, and T. Stockhammer, "Recepción móvil de TDT mediante protección AL-FEC," in XIX Jornadas Telecom I+D, Madrid, Spain, 2009.

[NC5] D. Gómez-Barquero et al., "DVB-NGH, la nueva generación de televisión digital móvil" in XXI Jornadas Telecom $I+D$, Santander, Cantabria, 2011.

\section{Contributions to Standardization Activities}

[ST1] 12 contributions to the MIMO task force in the standardization process of DVB-NGH (www.dvb.org).

[ST2] 1 contribution to the CCI task force in the standardization process of DVB-NGH (www.dvb.org).

[ST3] Contribution to the implementation guidelines of DVB-T2 [42] in clause 5.9: Choice of time interleaving parameters, clause 14: Performance, and clause 16: T2-Lite. 


\section{Papers Under Review}

[S1] D. Gozálvez, J. J. Giménez, D. Gómez-Barquero, and N. Cardona, "Rotated constellations for improved time and frequency diversity in DVBNGH," submitted to IEEE Transactions on Broadcasting.

[S2] D. Gozálvez, D. Gómez-Barquero, P. F. Gómez, and N. Cardona, "Combined Time, Frequency and Space Diversity in DVB-NGH" submitted to IEEE Transactions on Vehicular Technology.

[S3] D. E. Vargas, D. Gozálvez, D. Gómez-Barquero, and N. Cardona, "MIMO for DVB-NGH, the next generation of mobile TV broadcasting," submitted to IEEE Communications Magazine. 


\section{Chapter 2}

\section{Diversity Techniques in Mobile Broadcasting DVB Systems}

In this chapter we review the state of the art in diversity techniques for terrestrial broadcasting DVB systems. First, in Section 2.1, we explain the fundamentals of FEC and interleaving, which are used to achieve diversity in the time and frequency domains. Then, in Section 2.2, we present the main concepts regarding the utilization of multiple antennas, which can provide not only diversity but also array and multiplexing gains. In Section 2.3, we introduce rotated constellations and explain their utilization in DVB standards. In Sections 2.4, 2.5 and 2.6 we describe in detail the mechanisms that have been adopted in the current and next generation of terrestrial broadcasting DVB systems for time, frequency and space diversity. Finally, we conclude the chapter in Section 2.7 by summarizing the key concepts.

\subsection{FEC and Interleaving}

\subsubsection{Forward Error Correction}

FEC is a mechanism for error control designed to automatically correct errors detected at the receiver. This is accomplished by inserting controlled redundancy into the transmitted sequence of information, which allows the receiver to detect and correct a certain number of errors made during the transmission. The algorithm for computing this redundancy is commonly referred to as error 


\section{CHAPTER 2. DIVERSITY TECHNIQUES}

control code or error correcting code. In this section, we first present the two main categories of error control codes, namely block and convolutional codes. Then, we review the most important codes used in the DVB family of standards. In particular, we focus on RS, Raptor, LDPC and turbo codes. The first two types of codes are primarily used in UL-FEC mechanisms due to their good correction capabilities of erasures (i.e. known portions of information that are lost). LDPC and turbo codes on the other hand, have been included in the physical layer of DVB-T2 and DVB-SH respectively due to the fact that they can approach the Shannon's capacity bound in AWGN channels quite closely, and with reasonable decoding complexity.

Block codes process the information as chunks of data that are encoded one after another. The encoder of a binary block code divides the information sequence to be encoded into message blocks of $k$ source bits. Then, the encoder transforms each message independently into a codeword of $n$ bits. Because the $n$-bit output codeword depends only on the corresponding $k$-bit input message, i.e. each message is encoded independently, the encoder is said to be memoryless. If a so-called systematic code is applied, the $k$ original bits can be found among the $n$ bits that constitute the codeword, while the remaining $n-k$ bits are the parity bits. Linear block codes are an important subclass of block codes, as they allow for reduced encoding and decoding complexity. A linear block code is defined in terms of its generator and parity check matrices [13].

The error probability of FEC codes depends on whether the decoder employs hard decision or soft decision decoding. In the former case, the decoder only operates with binary inputs ( 0 or 1$)$, whereas in the latter case, the decoder accepts soft multilevel (or continuously valued) inputs. Although the utilization of soft decision decoding makes the decoder more difficult to implement, it offers a significant performance improvement compared to hard-decision decoding. It is generally stated that the advantage of soft decision decoding compared to hard decision decoding in terms of CNR is 2-3 dB [62]. Hard decision decoding of linear block codes uses minimum distance decoding based on Hamming distance, which is the number of coded bits that differ between different codewords or error events. The decoder compares the received codeword with the $2^{k}$ codewords that comprise the code and decides in favour of the codeword with the minimum Hamming distance to the received codeword. If there is more than one codeword with the same minimum Hamming distance, one of these is chosen at random by the decoder. This procedure can detect all the error patterns of $d_{m i n}-1$ bits and can correct up to $t=d_{m i n} / 2$ random errors, where $d_{\text {min }}$ is the minimum Hamming distance between codewords. For soft decision decoding, the euclidean distance between modulated symbols, rather than the Hamming distance, is a better metric of the error correction capabilities of the code. In fact, the best codes for the AWGN channel under soft decision 
decoding should focus on maximizing the euclidean distance between the codewords after modulation. This requires the channel code to be designed jointly with the modulation and is the basis for Coded Modulation (CM) [63]. On the contrary, the code performance in the presence of fading depends, rather than on the minimum Euclidean distance of the code, on its minimum Hamming distance (the code diversity). In this case, the use of BICM, which separates modulation from coding, generally achieves a better performance [64]. BICM has became the dominant technique in wireless communication systems and is the solution adopted in the DVB family of standards. In BICM systems, soft information indicates the probability that a bit was transmitted as a 1 or as a 0 , and is generally expressed in Log-Likelihood Ratio (LLR) form. Denoting by $\operatorname{Pr}\{b=1\}$ the probability that a bit $b$ has logical value 1 , the $\operatorname{LLR} L(b)$ about $b$ is given by:

$$
L(b)=\log \left(\frac{\operatorname{Pr}\{b=1\}}{1-\operatorname{Pr}\{b=1\}}\right),
$$

where the sign of $L(b)$ gives the hard decision about $b$ and the magnitude $|L(b)|$ measures the reliability of this decision.

On the other hand, convolutional codes process the information as a continuous stream of data and add parity constantly. The encoder of a convolutional code also accepts sequences of $k$ bits of information in order to produce encoded sequences of $n$ bits of information. However, each encoded block depends not only on the corresponding $k$-bit message block, but also on the $m$ previous message blocks. Hence, the encoder has a memory order of $m$. Convolutional codes are linear and can be defined by its generator polynomial. A very common way of representing convolutional codes is by means of a trellis diagram [13]. Unlike block codes, where decoding entails finding the most likely $n$-bit codeword that is closest to the received $n$-bit codeword, decoding of convolutional codes entails finding the most likely sequence of coded bits given the received sequence of coded bits. Perhaps the most important decoding algorithm for convolutional codes is the Viterbi algorithm. As with block codes, the error correction capabilities of convolutional codes depend on the minimum distance between sequences of coded bits, which in this case is referred to as the minimum free distance, $d_{\text {free }}$, of the code.

\section{Reed Solomon Codes}

RS codes were discovered by Reed and Solomon in 1960 [65] and can be considered as a subclass of a larger class of linear block codes known as Bose Chadhuri Hocquenghem (BCH) codes [13]. They are commonly used in DVB systems due to their good error detection capabilities and the fact that they 


\section{CHAPTER 2. DIVERSITY TECHNIQUES}

are very effective in correcting random symbol errors and random burst errors. RS codes are non binary codes; the symbols from each codeword are chosen from a non binary alphabet of size $q>2$. Usually $q=2^{b}$ so that $b$ bits of information can be mapped into one codeword symbol. Systematic RS codes are generally referred to as $\operatorname{RS}(N, K)$, where $N$ is the length of the code, i.e. the number of symbols that comprise each codeword, and $K$ is the number of source symbols. The length of a RS code is one less than the size of the code alphabet, so that $N=q-1$ and $K=1,2, \ldots, N-1$, where the value of $K$ dictates the error correction capabilities of the code. Specifically, a RS code can correct up to $t=(N-K) / 2$ random symbol errors, and up to $2 t$ errors if the receiver knows the location of the erroneous symbols.

Due to the encoding and decoding complexity of RS codes, they are practical only for small values of $N, K$ and $q$. The most commonly used RS code operates with symbols of eight bits (one byte), such that there is a direct translation between bytes and codeword symbols. A RS code operating on symbols of eight bits results in $N=255$ and $K<255$. Different code rates can be obtained from a mother $\operatorname{RS}(N, K)$ code by means of puncturing and padding. A weaker code rate is achieved with puncturing by discarding and not transmitting one or more of the last parity symbols. In contrast, a more robust code rate can be achieved by zero padding the last source symbols, yielding a so-called shortened RS code. These padding symbols are used only for generating the parity symbols but are not transmitted. In this case, decoders have to add the removed padding symbols before decoding the codewords.

\section{Raptor Codes}

Raptor codes were invented by Shokrollahi in 2000 as an extension of Luby Transform (LT) codes with linear time encoding and decoding [66]. They are commonly used in UL-FEC mechanisms due to their near optimal erasure correction capabilities and the fact that they can be implemented on software without the need of dedicated hardware even in handheld devices, which in turn, allows supporting a large number of source symbols. Raptor codes belong to a family of codes known as Fountain codes [67]. Compare to traditional block codes, Fountain codes are rateless in the sense that the number of encoded symbols that can be generated from $K$ source symbols is potentially limitless; and that the number of encoded symbols can be determined on the fly. At the receiver side, it is possible to recreate the source symbols as soon as a sufficient number of encoded symbols is received without errors, no matter which. Contrary to RS codes, which are optimal for erasure decoding, Raptor codes require a slightly higher amount of received symbols in order to recover the $K$ source symbols with very high probability. 
Compared to LT codes, Raptor codes achieve their computational superiority by applying a precode to the $K$ source symbols. By applying a precode, the LT decoder has to recover only a constant fraction of the source symbols, which simplifies the problem. The drawback is a higher reception overhead for small values of $K$. This can be explained by the fact that for small values the variance of the decoding process is too large, and hence the decoding fails more often. The latest iteration of Raptor codes, known as RaptorQ [68], employs a two-stage precoding algorithm based on LDPC and High Density Parity Check (HDPC) codes. The decoding probability of RaptorQ in practical applications is greater than $99 \%$ with $K$ correctly received symbols, greater than $99.99 \%$ with $K+1$ symbols and greater than $99.9999 \%$ with $K+2$ symbols [69].

\section{Turbo Codes}

Turbo codes were introduced in 1993 [17] by Berrou, Glavieux and Thitimajshima. A classical turbo code is formed by the parallel concatenation of two binary Recursive Systematic Convolutional (RSC) codes $C^{1}$ and $C^{2}$ referred to as the first and second constituent code, which are separated by a permutation (interleaving). Serial concatenation is also possible. Although the constituent codes are generally the same, this is not necessary for good performance. The utilization of RSC codes is a key component of turbo codes and offers several advantages as compared with classical convolutional codes such as a better performance at low values of CNR. In contrast to block codes, turbo codes are not a-priori well suited for the transmission of finite length sequences of information. The solution adopted in some standards forces the termination of at least one of the constituent codes in the all zero state, which is accomplished by inserting additional dummy tail bits at the end of the transmission sequence. With appropriate termination techniques such as tail-biting, it is possible to terminate the code without the need of dummy bits or any performance loss in the decoding process. In addition to tail-biting, it is also common to puncture some parity bits in order to produce higher code rates than the mother code.

Turbo decoding relies on the exchange of probabilistic messages between two soft-input soft-output decoders in an iterative manner [70]. Each decoder takes input LLR estimates about the transmitted bits and tries to improve these estimates using the local redundancy of the associated constituent code. The result of the decoder's estimation without taking into account its own input is referred to as extrinsic information, and is precisely what is exchanged between decoders in an iterative manner. After a given number of operations, the two decoders converge towards a stable final decision for each bit. In general, the decoding process continues until a particular stop criteria is fulfilled. 


\section{CHAPTER 2. DIVERSITY TECHNIQUES}

\section{Low Density Parity Check Codes}

LDPC codes were originally invented by Gallager in 1961 [71], but they were largely ignored for decades until they were rediscovered by Mackay and Neal in 1995 [16]. LDPC codes are linear block codes with a particular structure for the parity check matrix. In particular, the fraction of nonzero entries in the parity check matrix is very small (i.e. low density). A regular LDPC code has the following structural properties in its parity check matrix: each row consists of $d_{r}$ ones, each column consists of $d_{c}$ ones, and the number of ones in common between any two columns is not greater than one. In contrast, if all the columns or all the rows of the parity check matrix have not the same weight, the LDPC code is said to be irregular. LDPC codes are generally represented by a bipartite (Tanner) graph, in which $n$ message nodes are connected to $n-k$ check nodes according the parity check matrix of the code. The $n$ symbols of the codeword are associated with the message nodes so that for all check nodes, the modulo-2 sum of neighboring positions among the message nodes is zero. The number of connections or edges of each message node is referred to as the degree of that node. It must be noted that puncturing of LDPC codes is generally not advisable due to the different importance of the code bits for the decoding process.

Decoding of LDPC codes is generally performed by means of message passing and iterative algorithms based on belief propagation [72]. The reason for this name is that, at each round, messages are passed from message nodes to check nodes, and from check nodes back to message nodes. The messages from message nodes to check nodes are probabilities or beliefs, which are computed based on the observed value of the message node and some of the messages passed from the neighboring check nodes to that message node. The decoding iterations continue until the hard decision of the message nodes makes all the parity-check sums equal to zero, or until a certain number of iterations is reached. The belief propagation decoding of LDPC codes is easily parallelizable and can be closely approximated with low complexity decoders. In addition, it can detect whether a correct codeword is decoded at the end of each iteration.

\subsubsection{Interleaving}

To improve the performance of FEC mechanisms in fading channels, error control codes are generally combined with some form of interleaving [73]. The main idea of interleaving is to spread the codewords in the time and frequency domain so that the channel becomes memoryless to the decoder. In order to ensure an independent distribution of errors across codewords, the size of the interleaving must be large enough according to the correlation of the mobile 


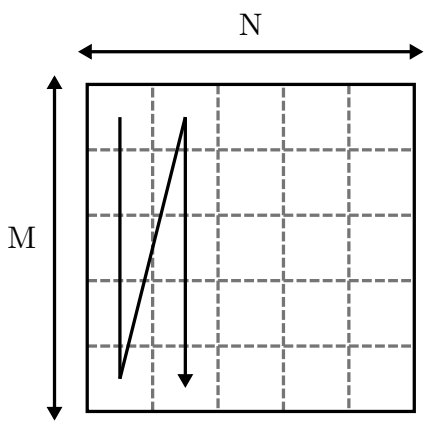

Interleaver

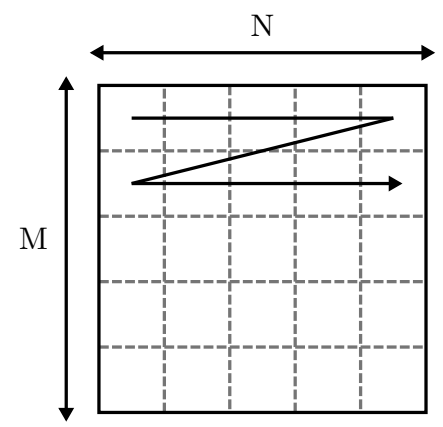

Deinterleaver

Figure 2.1: Block interleaver.

channel. It must be noted that the use of interleaving in reception scenarios where the average received CNR falls below the reception threshold not only does not improve the performance but it may actually degrade it [27]. As with error control codes, interleavers are generally divided into two main categories: block and convolutional interleavers [62]. A block interleaver can be represented as a $M$-row and $N$-column matrix (see Fig. 2.1). The information symbols prior to interleaving are written into the matrix column by column from left to right. Once the matrix is completely filled with information, the interleaved bits are read out from the matrix row by row and downwards. In the receiver side, the inverse operation is performed to retrieve the information symbols in their original order. If we assume that each column in the interleaving matrix is occupied by one codeword of $M$ symbols, an error burst affecting $N$ adjacent symbols of information would be distributed after deinterleaving as a single error per codeword. The end-to-end delay caused by the interleaving and deinterleaving processes is $2 M N$ symbol times. The zapping time depends in this case on the exact time of channel switching, and ranges from a maximum of $2 M N$ down to a minimum of $M N$, with an average value of $(3 M N) / 2$. The amount of memory required in both transmitter and receiver is $M N$ symbols. Although it may seem that two blocks of memory are needed, one for writing and one for reading, efficient implementations might require just one memory block [42].

Convolutional interleavers were originally proposed by Ramsey in 1970 [26]. Convolutional interleavers can be represented as a bank of parallel shift registers [73]. Fig. 2.2 illustrates a uniform $M \times N$ convolutional interleaver consisting of $N$ First-In First-Out (FIFO) shift registers from $i=1 \ldots, N-1$, where each register is $J$ symbols longer than the previous one, given $M=N J$. The 

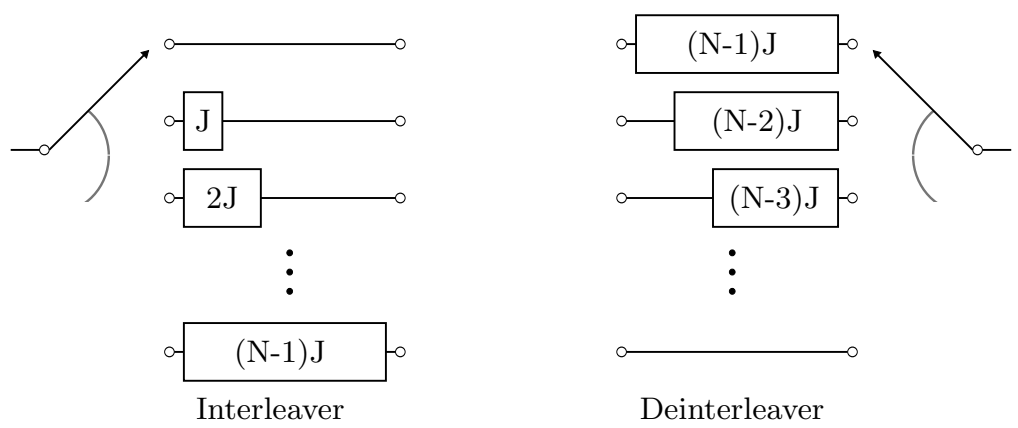

Figure 2.2: Convolutional interleaver.

first register is not actually a register, and the information symbols pass by without any delay. For each cycle of the interleaver, $N$ information symbols are read sequentially and written into the registers. The input and output of the registers is synchronized so that each time a new symbol is written into a register, the oldest symbol from the same register is read out. As a result of the interleaving process, the $i$-th symbol from each block is delayed by $(i-1) N J$ time units through a $(i-1) J$ register that is clocked once every $N$ symbol times. The end-to-end delay and memory requirements of convolutional interleavers are halved compared to block interleavers. Specifically, the end-to-end delay is $M(N-1)$ while the memory required at the transmitter and receiver is $M(N-1) / 2$. On the other hand, the zapping time does not depend on the instant of channel switching and is equal to $M(N-1) / 2$. Convolutional interleavers can also achieve a non-uniform interleaving of information depending on the number of registers and their delay. Although non-uniform interleaving configurations achieve a lower performance in mobile channels, they can be useful for reducing the zapping time.

\subsection{Multiple Antennas}

\subsubsection{Array, Diversity and Multiplexing Gains}

The utilization of multiple antennas in mobile broadcasting systems can provide array, diversity and multiplexing gains. Array gains are achieved by having the received signals coherently added. This results in a constant increase in terms of CNR that does not depend on the properties of the channel and that is only determined by antenna characteristics. With co-polarized antennas, the gain is equal to $3 \mathrm{~dB}$ every time the number of receive antennas is doubled. With cross-polarized antennas, the gain depends on the Cross Polarization 
Discrimination (XPD) as well as on the presence of polarization mismatch and asymmetry. Transmit beamforming strategies can also be used to provide an array gain by having the received signals from the various transmit antennas added up coherently, and by allocating more power to the transmit antennas with the better gains. However, transmit beamforming techniques require channel knowledge at the transmitter side, which is not available in broadcasting systems due to the lack of an uplink channel.

Diversity gains are achieved by averaging the fading across the propagation paths that exist between the transmit and the receive antennas. A MIMO scheme is said to have a diversity order of $d$ if the average error probability $P_{e}(\mathrm{CNR})$ as a function of the CNR behaves as [74]:

$$
\lim _{\mathrm{CNR} \rightarrow \mathrm{inf}} \frac{\log \left(P_{e}(\mathrm{CNR})\right)}{\log (\mathrm{CNR})}=-d
$$

This means that a MIMO scheme with diversity order $d$ has an error probability of $P_{e}(\mathrm{CNR}) \approx \mathrm{CNR}^{-\mathrm{d}}$ at high $\mathrm{CNR}$ values. A MIMO system with $M_{t}$ transmit and $M_{r}$ receive antennas potentially offers $M_{t} M_{r}$ independent paths and hence, a diversity order of $M_{t} M_{r}$. MIMO schemes that increase the reliability of information through diversity are often collectively referred to as transmit diversity. In the case of co-polarized antennas, the diversity gain depends on the coherence distance of the channel. This is defined as the maximum spatial separation over which the channel can be assumed constant, and is characterized by the angular spread, which depends on the local scattering environment and the carrier frequency. In order to ensure independent fading across antennas, it is necessary that the separation between antennas is larger than the coherence distance. For a mobile which is near the ground with many scatterers around, the channel decorrelates over shorter space distances, and typical antenna separations of half to one carrier wavelength are sufficient. For transmit towers, larger antenna separations of several to tens of wavelengths may be required as a result of the longer distance between the scatterers and the antennas [31]. Cross-polarized antennas on the other hand, rely on the fact that the fading experimented by each polarization path possesses a low degree of correlation. The diversity gain in this kind of systems depends not only on the correlation between polarization paths, but also on additional factors such as the XPD. In particular, higher values of XPD have been shown to be detrimental for transmit diversity [30].

The simultaneous utilization of multiple antennas at the transmitter and receiver side permits the opening of multiple spatial pipes across the wireless channel. In this case, multiplexing gains are achieved by multiplexing independent data streams across the spatial pipes. A MIMO scheme is said to have 


\section{CHAPTER 2. DIVERSITY TECHNIQUES}

a multiplexing gain of $r$ if the transmission rate $R(\mathrm{CNR})$ as a function of the CNR behaves as [74]:

$$
\lim _{\mathrm{CNR} \rightarrow \text { inf }} \frac{R(\mathrm{CNR})}{\log (\mathrm{CNR})}=r .
$$

This means that a MIMO scheme with multiplexing gain $r$ has a transmission rate of $R(\mathrm{CNR}) \approx \operatorname{rlog}(\mathrm{CNR})$ at high $\mathrm{CNR}$ values. A MIMO system with $M_{t}$ transmit and $M_{r}$ receive antennas potentially offers $\min \left(M_{t}, M_{r}\right)$ spatial pipes and hence, a multiplexing gain of $\min \left(M_{t}, M_{r}\right)$. MIMO schemes that increase the transmission rate of information are often collectively known as spatial multiplexing. In the case of co-polarized antennas, spatial multiplexing relies on independent fading between antennas to separate the multiple data streams at the receiver side. The presence of correlation, specially at the transmitter side, is very detrimental for spatial multiplexing techniques. In this case, the utilization of cross-polarized antennas, which rely not only on a low correlation between polarization paths, but also on the decoupling between polarizations to separate the data streams, generally improves the performance [30].

The so-called Diversity-Multiplexing Tradeoff (DMT) describes the relationship between diversity and multiplexing in MIMO systems as the $(d, r)$ pairs that are achievable for $\mathrm{CNR} \rightarrow$ inf. The optimum DMT frontier $d^{\text {opt }}(r)$ is defined as the maximum diversity gain that is achievable by any scheme with multiplexing gain $0 \leq r \leq \min \left(M_{t}, M_{r}\right)$ :

$$
d^{o p t}(r)=\left(M_{t}-r\right)\left(M_{r}-r\right) .
$$

Each MIMO scheme is associated to a particular trade-off $(d, r)$ that may be smaller than the optimum DMT frontier shown in Eq.(2.4). This trade-off indicates the speed at which the error probability decreases with the CNR: if the rate is increased as $r \log (\mathrm{CNR})$, then the error probability decreases as $\mathrm{CNR}^{-\mathrm{d}(\mathrm{r})}$ [75]. A vast amount of SFBCs has been proposed in the literature with different diversity and multiplexing capabilities. The Alamouti code [31] is a well known SFBC that sacrifices multiplexing in order to achieve full diversity. Although this strategy is suboptimal in MIMO channels, the Alamouti code achieves the optimal DMT frontier in the $2 \times 1$ MISO channel. Another very representative SFBC is the Golden Code (GC) [76], which achieves the optimum DMT frontier in $2 \times 2$ MIMO systems.

\subsubsection{MIMO Demodulation}

The utilization of MIMO increases the complexity of receivers not only due to the implementation of multiple antennas, but also because of the signal processing that is required to demodulate the received signal. For a given channel 
realization, the received signal in a MIMO fading channel can be represented as:

$$
\mathbf{y}=\mathbf{H} \mathbf{x}+\mathbf{n},
$$

where $\mathbf{y}$ is the received signal vector, $\mathbf{H}$ is the $M_{r} \times M_{t}$ channel matrix, $\mathbf{x}$ is the transmitted signal vector, and $\mathbf{n}$ is the AWGN noise vector. A soft Maximum A Posteriori (MAP) demodulator calculates the LLR $L(b)$ of the code bit $b$ according to:

$$
L(b)=\log \frac{\sum_{x \in \chi_{b}^{1}} \exp \left(-\frac{\|\mathbf{y}-\mathbf{H} \mathbf{x}\|^{2}}{\sigma_{w}^{2}}\right)}{\sum_{x \in \chi_{b}^{0}} \exp \left(-\frac{\|\mathbf{y}-\mathbf{H} \mathbf{x}\|^{2}}{\sigma_{w}^{2}}\right)},
$$

where $\sigma_{w}^{2}$ is the noise variance and $\chi_{b}^{l}$ denotes the set of transmitted vectors for which $b=l \in\{0,1\}$. Although the solution in Eq.(2.6) is optimal in the sense that obtains exact LLRs, it requires the computation of $A^{M_{t}}$ distances, where $A$ represents the symbol cardinality. Therefore, the complexity of soft MAP demodulation grows exponentially with the number of transmit antennas, which may restrict its implementation to small signal constellations and small numbers of antennas. For this reason, a large number of suboptimal demodulators with reduced complexity have been proposed. They can be categorized in non-linear and linear demodulators depending on performance and complexity characteristics [77]. Perhaps the most representative example of non-linear suboptimal demodulators is sphere decoding [78, 79]. The basic idea of sphere decoding is to restrict the search in the Maximum Likelihood (ML) detection problem to a smaller subset of potential candidates. Although this strategy achieves near ML performance and reduces the complexity in a significant manner, it still grows exponentially with the number of transmit antennas. Linear demodulators on the other hand, preprocess the received signal with a linear filter to separate the transmitted symbols. In this case, the complexity does not increase exponentially but linearly with the number of transmit antennas. Two classical approaches exist for linear demodulation: Zero Forcing (ZF) and Minimum Mean Square Error (MMSE) demodulation. The filter matrix $G$ of the $\mathrm{ZF}$ equalizer is given by [80]:

$$
\mathbf{G}^{\mathbf{Z F}}=\left(\mathbf{H}^{\mathrm{H}} \mathbf{H}\right)^{-1} \mathbf{H}^{\mathrm{H}},
$$

whereas the MMSE equalizer is given by [80]: 


$$
\mathbf{G}^{\mathbf{M M S E}}=\left(\mathbf{H}^{\mathrm{H}} \mathbf{H}+\frac{M_{t}}{\mathrm{CNR}} \mathbf{I}\right)^{-1} .
$$

It should be pointed out that the utilization of ZF completely removes the effect of $\mathbf{H}$ at the expense of noise enhancement. In contrast, the MMSE filter balances $\mathbf{H}$ mitigation with noise enhancement in order to minimize the total error.

\section{$2.3 \quad$ Rotated Constellations}

Rotated constellations exploit the concept of SSD to increase the diversity order of BICM systems at the expense of higher demodulation complexity without the need of additional transmission power or bandwidth. The main idea behind SSD is to apply a certain rotation to the information symbols in order to spread the binary information across a higher number of components, with each component experiencing independent fading.

Two-Dimensional Rotated Constellations (2DRC) [81] were introduced in DVB-T2 to improve the reception robustness in fading scenarios and Single Frequency Networks (SFN). With regular, non-rotated constellations, each real component in-phase (I) or quadrature (Q) carries half of the binary information held in the Quadrature Amplitude Modulation (QAM) symbol. When the signal is subject to severe fading, both components experience identical degradation, and the information carried in the QAM symbol suffers an irreparable loss. In the case of 2DRC (see Fig. 2.3), each I and Q component carries all the binary information hold in the rotated symbol. By ensuring that each component experiments an independent fading realization, the binary information can be retrieved even if the rotated symbol is deeply faded or erased. To this purpose, the I and Q components of two different QAM symbols are swapped and transmitted in different subcarriers. The use of 2DRC improves the reception robustness against fading but requires a higher demodulation complexity, since the I and Q components of each QAM symbol must be demodulated together. The rotation in DVB-T2 has been optimized for each constellation as a good compromise between the pairwise error probability over fading channels in high CNR regions, and the bit error probability when one of the components I or $\mathrm{Q}$ is erased [81].

DVB-NGH has inherited the 2DRC of DVB-T2, and has also included Four-Dimensional Rotated Constellations (4DRC) for certain configurations (Quadrature Phase-Shift Keying (QPSK) and code rates higher than 1/2). With $4 \mathrm{DRC}$, the rotation is applied to pairs of information symbols in order to spread the binary information carried in two symbols across four different 


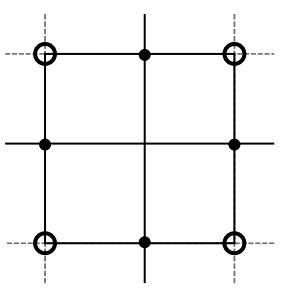

QPSK

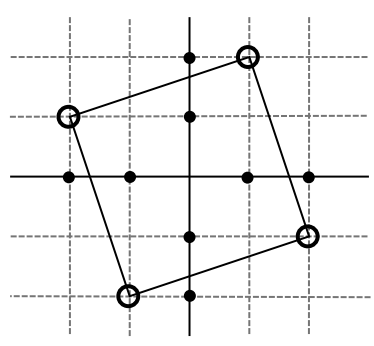

QPSK 2DRC

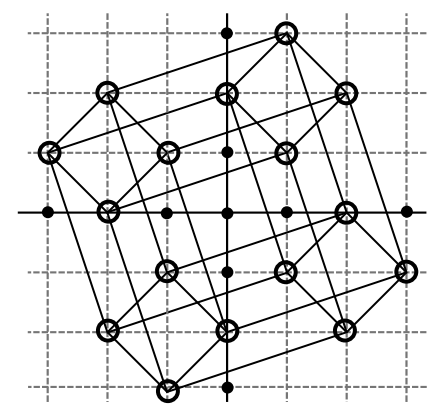

QPSK 4DRC

Figure 2.3: Two-dimensional and four-dimensional rotated constellations.

components. In this case, the rotation has been selected to minimize the bit error probability according to physical layer simulations in Rayleigh channels. Increasing the dimension of the rotation improves the robustness against fading, but requires a higher demodulation complexity due to the fact that all the components of the rotated symbols must be demodulated together in order to retrieve the binary information [82].

\subsection{Time Diversity Techniques}

\subsubsection{Time Interleaving}

In order to achieve time diversity, it is desirable that the interleaving duration in the time domain is longer than the coherence time, which can be defined as the period of time over which the propagation channel remains constant. In the case of fast fading, the coherence time of the channel is inversely proportional to the Doppler spread, which determines the rate of change of the signal amplitude over time as a function of the carrier frequency and the user velocity ${ }^{1}$. On the other hand, the coherence time of shadowing is characterized by the user velocity and the correlation distance $d_{c o r r}$, defined as the distance over which the normalized autocorrelation falls to $0.37\left(e^{-1}\right)^{2}$. While interleaving durations

\footnotetext{
${ }^{1}$ The coherence time of fast fading channels can be approximated by $T_{c} \approx 9 /\left(16 \pi f_{m}\right)$, where $f_{m}$ is the Doppler spread. In turn, the Doppler spread is given by $f_{m}=f_{c}(v / c) \cos \alpha$, where $f_{c}$ is the carrier frequency, $v$ is the user velocity, $c$ is the speed of light, and $\alpha$ is the angle of arrival with respect to the direction of motion.

${ }^{2}$ The coherence time of shadowing channels can be approximated by $T_{c} \approx d_{\text {corr }} / v$, where $v$ is the user velocity and $d_{c o r r}$ is the correlation distance. Therefore, the coherence time is defined as the period of time that a mobile user employs for traveling the correlation distance.
} 


\section{CHAPTER 2. DIVERSITY TECHNIQUES}

in the order of hundreds of milliseconds are generally sufficient for fast fading, several seconds of interleaving may be required in the presence of shadowing.

The utilization of TI increases the end-to-end delay as a result of the interleaving and deinterleaving that take place at the transmitter and the receiver sides. In Section 2.1 we have seen that the end-to-end delay introduced by TI is approximately equal to the interleaving duration in the case of convolutional interleavers and equal to two times the interleaving duration with block interleavers. In addition to end-to-end delay considerations, TI has also some implications in power saving and zapping time that we detail next.

\section{Considerations on Zapping Time}

Zapping time has a significant impact on the user's Quality of Experience (QoE) [28]. Values around $1 \mathrm{~s}$ are usually considered as satisfactory, whereas more than 2 seconds are felt as annoying [83]. Some studies show that the perceived QoE drops sharply if the zapping time is too high. In particular, the QoE does not decrease linearly with the zapping time, but follows a logarithmic trend instead [84]. As a result, long interleaving durations (e.g. $10 \mathrm{~s}$ ) cannot be generally achieved with tolerable zapping times unless fast zapping techniques are used. With fast zapping, receivers in good reception conditions can start displaying the service without waiting for the entire codewords to arrive. This is usually known as early decoding. From the point of view of the FEC decoder, the decoding of partial codewords is seen as some puncturing that reduces the error correction capabilities of the code. Therefore, receivers in bad channel conditions may have no other choice but to wait until the reception of all the source and parity symbols before the codewords can be successfully decoded. This is usually referred to as late decoding. Depending on how the parity and source symbols have been arranged for transmission, a receiver that has performed early decoding may transition to late decoding by means of parity recovery techniques [85].

In order to perform fast zapping it is important that the FEC code possesses a high robustness against puncturing; this is the case of RS, Raptor and turbo codes. In contrast, LDPC codes exhibit a much lower performance in the presence of puncturing, and in situations of heavy puncturing, the codewords may not be decodable at all even for very large CNR values. Fast zapping also benefits from Uniform-Late (UL) interleaving as shown in Fig. 2.4. The main idea is to produce bursts with a reduced interleaving duration and a large amount of information. This way, receivers can attempt to perform early decoding just after receiving one of these late bursts. It should be pointed out that the utilization of UL interleaving is detrimental for the performance of late decoding as it reduces the overall time diversity [43]. 


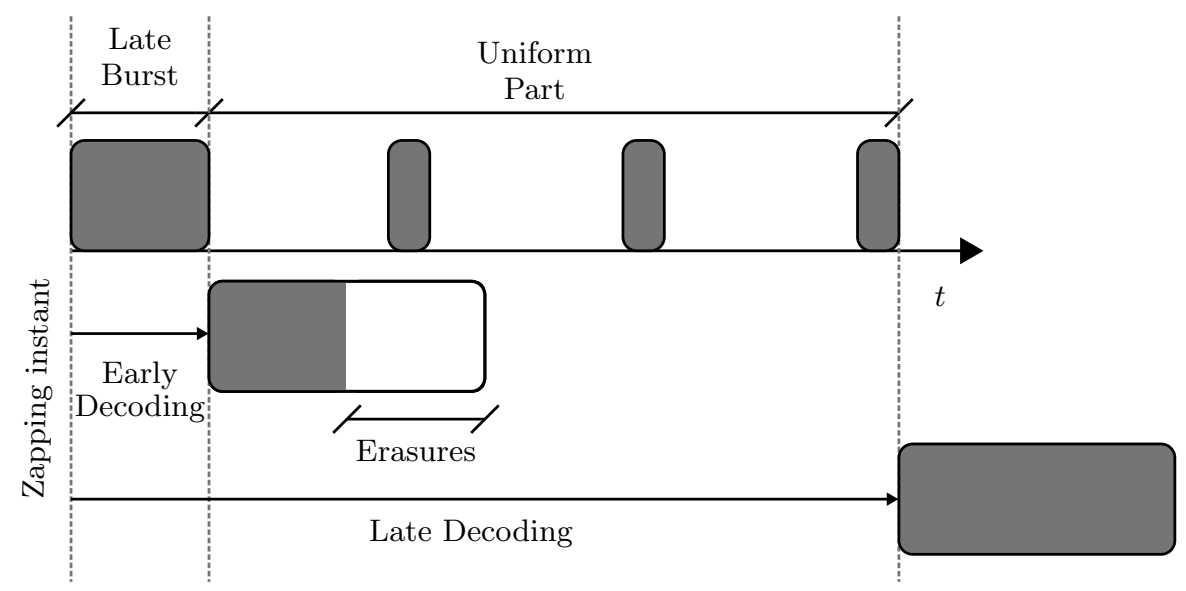

Figure 2.4: Uniform-late interleaving.

\section{Considerations on Power Saving}

Mobile broadcasting systems generally employ a discontinuous transmission of services in order to improve power saving in receivers. In the context of DVB-H and DVB-SH this is known as time slicing [86]. As it can be seen in Fig. 2.5, the information from each service is grouped and transmitted in time slicing bursts so that receivers can achieve power saving by switching off the RF part during the periods between bursts. The time that passes between the beginning of one burst and the next is referred to as the cycle time. Therefore, power saving $P_{s}$ in time slicing transmissions can be approximated by the following equation:

$$
P_{s} \approx 100 \times\left(1-\frac{T_{b}}{T_{c}}\right),
$$

where $T_{b}$ is the duration of each burst and $T_{c}$ is the cycle time. A classic example of time slicing employs bursts in the order of 100-200 ms and a cycle time in the order of 1-2 s, which results in a power saving of approximately $90 \%$.

The utilization of time slicing results in a trade-off between power saving and time diversity. From the point of view of power saving, the services should transmitted in as few bursts as possible. The main reason for this is that due to synchronization and channel estimation issues, receivers need to wake up a certain period of time before the actual reception of each burst. On the other hand, a more regular transmission of bursts is preferable for time diversity purposes. In particular, the maximum time diversity is achieved when 


\section{CHAPTER 2. DIVERSITY TECHNIQUES}

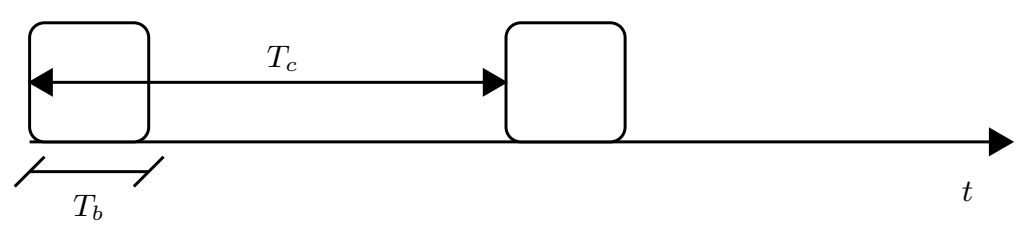

Figure 2.5: Time slicing.

services are continuously transmitted over time and time slicing is not used. The utilization of time slicing also affects the zapping time, since receivers must wait until the arrival of the first burst when switching to a new channel. The increase in terms of zapping time that is caused by time slicing is on average half the duration of the cycle time. This means that configurations with longer bursts and longer cycle times provide better power saving but result in longer channel switching times.

\subsubsection{UL-FEC vs. PL-FEC}

FEC mechanisms can be categorized into those working at the physical layer and those working at upper layers. PL-FEC mechanisms provide the best performance by working at the bit level and using soft decision decoding at the receiver side. However, this increases the memory requirements as it requires the storage of several bits per LLR in addition to some bits for Channel State Information (CSI). As a result, the maximum interleaving duration is constrained by the amount of on-chip memory available in receivers, and long TI at the physical layer is generally achieved with an external TDI memory.

UL-FEC mechanisms operate above the PL-FEC to provide longer interleaving durations with reduced memory requirements. In particular, UL-FEC recovers from packet errors by means of erasure decoding. This means that each packet is treated at the input of the FEC decoder as completely correct or entirely lost. Therefore, it is necessary to indicate whether each packet is correctly received or not (e.g. with checksums such as Cyclic Redundancy Check (CRC)), so that the UL-FEC decoder sees a packet erasure channel. Despite the fact that the packet erasure decoding performed by UL-FEC techniques is suboptimal compared to the soft decision decoding performed in PL-FEC, the performance loss in on-off channels is very much reduced. These channels are characterized by the correct reception or the complete loss of large contiguous portions of information, which approximates the packet erasure channel that is seen at upper layers. A very representative example of an on-off channel is the satellite railway scenario. 
The utilization of UL-FEC results in a cross-layer FEC optimization problem that is more difficult to solve. The main idea of using UL-FEC is to sacrifice performance in stationary channels so that the overall robustness in mobile channels is increased as a result of the longer interleaving duration. UL-FEC techniques do not improve the reception in stationary channels, and actually degrade the performance compared to having all the protection in the physical layer.

\subsubsection{UL-FEC Techniques}

Different UL-FEC mechanisms are available in DVB specifications. Depending on whether these mechanisms are integrated in the application layer above the IP level or in the link layer below the IP level, they are categorized as AL-FEC or Link Layer - Forward Error Correction (LL-FEC) respectively. We now present the UL-FEC mechanisms that are typically used in DVB systems for the protection of streaming services in mobile channels.

\section{MPE-FEC}

MPE-FEC is an optional LL-FEC mechanism that was developed during the standardization process of DVB-H in order to compensate for the lack of TI in DVB-T and improve the robustness against fast fading and impulse interference. MPE-FEC is an intra-burst FEC mechanism in the sense that the protection is limited to the duration of one time slicing burst. Although this is generally sufficient against fast fading, MPE-FEC cannot recover from the loss of entire bursts of information. MPE-FEC is introduced in such a way that MPE-FEC ignorant DVB receivers are still able to receive the source information in a fully backward compatible way.

MPE-FEC operates at the link layer by protecting the Internet Protocol (IP) datagrams by means of a $\mathrm{RS}(255,191)$ code and a block interleaver. The encoding process is as follows. IP datagrams are written into a matrix, known as the MPE-FEC frame, column by column. The matrix consist of 255 columns and a flexible number of rows (maximum 1024). Each position in the matrix represents a single byte of information. Among the 255 columns, 191 are for IP information and 64 are for parity data. The parity columns are generated by encoding the information of the MPE-FEC matrix row by row. After that, the IP datagrams and the parity columns are encapsulated into MPE sections and transmitted in time slicing bursts. Within the bursts, the MPE sections with IP datagrams are transmitted in the same order as they were generated, and before the MPE sections with parity columns. At the receiver side, a CRC field in the MPE sections indicates whether each section has been correctly received 
or not so that the receiver can perform erasure decoding in the MPE-FEC frame.

\section{MPE-iFEC}

MPE-iFEC was developed in the standardization process of DVB-SH to support reception in the presence of long signal outages spanning several time slicing bursts. Such situations are characteristic in LMS scenarios due to the common presence of obstacles in the LoS between the satellite and the receiver. MPE-iFEC is an inter-burst FEC mechanism that extends the interleaving duration across several bursts so that it is possible to recover from the loss of entire bursts. As with MPE-FEC, the utilization of MPE-iFEC is optional and fully backward compatible with receivers not supporting MPE-iFEC operation. MPE-iFEC is defined as a generic multi-burst framework that supports a several configurations and mappings. Until now, two different mappings have been defined in MPE-iFEC: one mapping based on the same RS $(255,191)$ code employed in MPE-FEC, and another mapping based on Raptor codes. While the mapping based on RS codes is mandatory for the utilization of MPE-iFEC, the mapping based on Raptor codes is left as optional.

The mapping based on RS employs a sliding window approach in order to extend the protection across multiple bursts. The sliding window interleaves the IP datagrams from $B$ different bursts of information into $B$ encoding matrices in a progressive manner. Once an encoding matrix has been filled with information, the parity is generated by the RS code in a similar manner to MPE-FEC. The parity columns from each encoding matrix are then interleaved across $S$ time slicing bursts. IP datagrams and parity columns are encapsulated into MPE sections and transmitted in time slicing bursts. IP datagrams can be delayed $D$ bursts before transmission in order to facilitate the utilization of fast zapping at the receiver side. On the other hand, the mapping based on Raptor codes relies in the utilization of very large message blocks to extend the interleaving duration across multiple bursts. A single encoding matrix is filled with entire bursts of IP information that are encoded by the Raptor code as one codeword. The number of bursts that are encoded in the same codeword is determined by the encoded period EP.

\section{AL-FEC}

AL-FEC was originally adopted to be used instead of MPE-FEC to increase the robustness of file delivery services in DVB-H. In particular, AL-FEC uses the systematic Raptor code that is defined in the Internet Protocol Datacast (IPDC) Content Delivery Protocols (CDP) specification [48]. Although AL-FEC is currently not standardized for streaming services in IPDC, it could 
be implemented with some minor changes in mobile broadcasting DVB systems such as DVB-H or DVB-SH [46] by following the FEC streaming framework defined by the Internet Engineering Task Force (IETF) and already adopted by Third Generation Partnership Program (3GPP) Multimedia Broadcast Multicast Services (MBMS) [87]. Alternative implementations of AL-FEC based on Moving Picture Experts Group 2 (MPEG-2) Transport Stream (TS) packets could be also used in terrestrial broadcasting DVB systems like DVB-T or DVB-T2 [88]. Basically, the main idea of using AL-FEC for streaming services is to protect a large number of application layer packets as a single message block that can extend across several seconds in time. This is similar to the Raptor mapping defined for MPE-iFEC, and only some implementation and signaling specific aspects would differ.

\subsubsection{PL-FEC Techniques}

Although every DVB standard includes some kind of PL-FEC in order to combat channel noise, only DVB-SH and DVB-T2 incorporate TI at the physical layer. In particular, time diversity in DVB-T2 is achieved by means of LDPC codes and a block interleaver, whereas DVB-SH implements a turbo code and a combination of Block Interleaving (BI) and Convolutional Interleaving (CI). The next generation standard DVB-NGH will also incorporate both kinds of interleaving in order to achieve time diversity at the physical layer.

\section{PL-FEC with Block and Convolutional Interleaving in DVB-SH}

DVB-SH incorporates the turbo code standardized by the 3GPP2 organization [7]. It employs two RSC encoders connected in parallel, with an interleaver preceding the second recursive convolutional encoder. As it is usual with turbo codes, the different code rates included in the standard are achieved by means of puncturing. An output tail bit sequence is also added at the end of each codeword. On the other hand, TI is performed by using a block and a convolutional interleaver. The convolutional interleaver consists of $48 \mathrm{shift}$ registers with variable length that take as input a sequence of non-interleaved Interleaving Units (IU) of 126 bits each. The loss of one or more IU results in the presence of bursty errors on a bit level at the input of the FEC decoder, which may degrade the performance of the turbo code. As a result, a bit-wise block interleaver is placed between the FEC encoder and the convolutional interleaver in order to maximize the distance between erased bits within each codeword.

The length of the 48 shift registers that constitute the convolutional interleaver can be configured according to a series of parameters to create different 


\section{CHAPTER 2. DIVERSITY TECHNIQUES}

interleaver profiles. While the long uniform profile achieves the best performance, UL profiles might improve fast zapping at the expense of worse time diversity. These profiles also allow for a progressive recovery of the codewords after early decoding so that it is possible to achieve late decoding performance over time. A typical configuration of the UL profile has a target code rate of $4 / 5$ for the late part to enable successful decoding in good reception conditions.

Two different categories of terminals are distinguished in DVB-SH depending on their TI capabilities [43]. Class 1 receivers are equipped with approximately $4 \mathrm{Mb}$ of memory and can provide a maximum interleaving duration between $100 \mathrm{~ms}$ and $240 \mathrm{~ms}$. Class 2 receivers implement approximately $256 \mathrm{Mb}$ of memory and can provide a maximum interleaving duration between $15 \mathrm{~s}$ and $30 \mathrm{~s}$. The first class of receivers are designed to operate with MPE-iFEC at the link layer, since they do not implement the memory required for long TI at the physical layer. Although the utilization of UL-FEC reduces the overall performance compared to full PL-FEC protection, class 1 receivers generally represent a better compromise between hardware complexity and error correction capabilities. In contrast, the second class of receivers is expected to achieve a better performance at the expense of an external TDI memory.

\section{PL-FEC with Block Interleaving in DVB-T2}

At the FEC level, DVB-T2 inherits the coding scheme from the Digital Video Broadcasting - Satellite 2nd Generation (DVB-S2) standard based on the concatenation of LDPC and BCH codes. These are defined for six different code rates and two codeword lengths. Similarly to DVB-SH, the interleaving of the codewords is carried out in several stages. The time interleaver consists in a block interleaver that operates on a cell level. Each cell represents a complex symbol that is transmitted in a different subcarrier within the OFDM symbols. The time interleaver takes as input groups of cells referred to as TI blocks that are interleaved by the time interleaver one after another. After TI, the cells are mapped to T2 frames for transmission. The utilization of irregular LDPC codes in DVB-T2 results in a non-uniform error protection of each code bit, as it depends on the column weight of the parity check matrix. In a similar manner, the reliability of the bits in the case of multi-level constellations is not uniform either. For the purpose of controlling the correspondence between code bits and constellation points, a bit interleaver is placed between the LDPC encoder and the symbol mapper. In the case of rotated constellations, a cyclic delay of the imaginary part is applied to the constellation symbols right after the symbol mapper so that the real and imaginary parts end up in different cells. After this, a cell interleaver shuffles the cells within each block in a pseudo-random manner. 
DVB-T2 introduces the concept of Physical Layer Pipe (PLP) in order to provide per-service specific robustness. Each PLP is a virtual container that can be configured with a particular set of transmission parameters, including the constellation, the code rate and the TI. This way it is possible to accommodate multiple use cases, i.e. stationary, portable and mobile, in the same frequency channel. If there are more than one PLP, the data channels are multiplexed in time slices within the T2 frames, with each PLP generally carried in one time slice per frame. In this case, sub-slicing, frame skipping, and interframe interleaving can be used when mapping the PLPs to the T2 frames for transmission. With sub-slicing, the PLPs are carried in multiple sub-slices per frame. This accomplishes a more uniform distribution of information over time and improves time diversity. Frame skipping is a mechanism by which the PLPs are transmitted only in a subset of $\mathrm{T} 2$ frames regularly spaced over time, which gives potential for better power saving at the expense of worse time diversity. The interval between successive frames with service information is known as the frame interval. Lastly, inter-frame interleaving extends the transmission of each TI block beyond one T2 frame and across multiple frames. It must be noted that the maximum interleaving duration is limited by the amount of TDI memory that is implemented in receivers. This has been set to approximately $2^{19}$ cells for DVB-T2 and $2^{18}$ cells for the T2-Lite profile. Assuming that a cell requires two 10-bit values (for real and imaginary) together with 4 bits for CSI, the memory size is around $12 \mathrm{Mb}$ and $6 \mathrm{Mb}$ for DVB-T2 and the T2-Lite profile respectively. In addition to memory issues, TI in DVB-T2 is also limited by the zapping time. Due to the uniform interleaving achieved by the block interleaver and the bad performance of irregular LDPC codes against random puncturing, fast zapping is not supported in DVB-T2, and the zapping time is approximately 1.5 times the interleaving duration on average.

\section{PL-FEC with Block and Convolutional Interleaving in DVB-NGH}

For channel coding, DVB-NGH has adopted some LDPC codes inherited from DVB-T2 together with new LDPC codes in order to achieve a better granularity between different code rates (e.g. $1 \mathrm{~dB}$ step in AWGN). For TI, the standard has adopted a combination of intra-frame BI and inter-frame CI. When the TI extends across $N$ frames, each FEC block is partitioned in $N$ IUs that are delayed from 0 up to $N-1$ frames in a convolutional manner. After this, the information symbols of different IUs are interleaved uniformly within each NGH frame. Compared to DVB-T2, the solution adopted in DVB-NGH halves the end-to-end latency and doubles the maximum interleaving duration that is supported by receivers. In the hybrid terrestrial-satellite profiles, the standard has incorporated UL configurations of inter-frame interleaving similar to the 
UL profiles of DVB-SH. This enables lower zapping times with moderate to long interleaving durations at the expense of worse time diversity. In addition to inter-frame interleaving, frame skipping can be used for power saving, and sub-slicing for better time diversity. Since DVB-NGH retains the concept of PLPs for the transmission of data, the configuration of Modulation and Coding (MODCOD) and TI can be particularized on a service basis. DVB-NGH also maintains the bit and cell interleaving of DVB-T2, whereas it replaces the cyclic Q delay with a component interleaver. The main purpose of the component interleaver is to optimize the distribution of components with rotated constellations in the time and frequency domains. This provides a significant performance increase with long TI and TFS compared to the cyclic Q delay of DVB-T2.

DVB-NGH distinguishes between terrestrial and hybrid receivers in terms of TI capabilities. Terrestrial receivers implement the same on-chip memory as T2-Lite receivers whereas hybrid receivers make use of the on-chip memory only for intra-frame interleaving, and implement a larger external memory for inter-frame interleaving purposes. The utilization of adaptive cell quantization reduces the number of bits that are used for each real and imaginary part in the case of low order constellations, allowing a higher number of cells to be stored in receivers. In particular, the number of cells that can be stored in the on-chip memory of DVB-NGH receivers is $2^{19}$ cells for QPSK and 16QAM constellations, and $2^{18}$ cells for the rest. The external memory allows hybrid receivers to store $2^{22}$ cells for QPSK and 16QAM constellations (higher constellations are not supported for satellite transmission).

\subsection{Frequency Diversity Techniques}

\subsubsection{Frequency Interleaving}

Frequency diversity is achieved in broadcasting systems by means of COFDM. This combines the utilization of OFDM and FEC so that it is possible to recover from the loss of subcarriers caused by frequency selective fading. In addition, frequency interleaving is used to maximize the frequency diversity by spreading each codeword across the entire signal bandwidth, which is generally limited to one RF channel. In particular, it is desirable that the interleaving in the frequency domain is longer than the coherence bandwidth of the channel. Similarly to the coherence time, the coherence bandwidth can be defined as the bandwidth over which the channel is assumed constant, so that subcarriers separated longer than the coherence bandwidth experiment independent fading. The coherence bandwidth of the channel is inversely proportional to the delay spread, which defines the multipath nature of the channel as a func- 
tion of the relative delays and powers of the taps ${ }^{3}$. The utilization of COFDM and frequency interleaving in broadcasting systems generally achieves sufficient frequency diversity in the $8 \mathrm{MHz}$ channels of the UHF band [35, 36].

In addition to the frequency selectivity caused by multipath propagation, large differences in the received signal strength can be found across frequency channels. Field measurements performed by Teracom, the Swedish terrestrial broadcast network operator, indicated that the reception field strength varies strongly with the position of the receive antenna relatively to the transmitter [23]. The first source for large differences in the received signal strength is the frequency dependency of the transmitter and the receiver antenna diagrams. The antennas located in the transmitters are designed to cover the whole range of used $\mathrm{RF}$ channels while achieving an omni-directional radiation pattern in the horizontal plane. In the case of terrestrial broadcasting networks, this range covers the UHF bands IV/V $(470 \mathrm{MHz}-862 \mathrm{MHz})$. In practice, the design of the antenna is a compromise between the operation bandwidth and the uniformity of the horizontal radiation pattern. Actual transmit antennas present variations of the horizontal radiation pattern in the order of 3-6 dB that are also dependent on the frequency and the azimuth. As a result, users moving across the coverage area may experience strong signal variations depending on the RF channel and their relative position compared to the transmitter. Additional factors resulting in large signal variations across RF channels include the dependency of the wave propagation with the frequency or the physical and electrical characteristics of the antenna itself. In general, the lower the channel in the UHF band, the better the coverage over the service area. TFS is a novel technique included in DVB-T2 and DVB-NGH that extends the frequency interleaving across multiple RF channels so that it is possible to average the signal variations between channels [50].

\subsubsection{Time-Frequency Slicing}

Traditionally, broadcasting services are grouped into several multiplex before being transmitted over the air, with each multiplex being allocated to one RF channel. When TFS is used, the services are multiplexed across several RF channels by means of frequency hopping and time slicing (see Fig. 2.6). In particular, the time slices of each service are transmitted sequentially in the RF channels that constitute one TFS multiplex. The utilization of TFS offers

\footnotetext{
${ }^{3}$ The coherence bandwidth of the channel for a classical Doppler spectrum [20] of all components and a correlation of 0.5 is $B_{c}=\sqrt{3} /\left(2 \pi \tau_{R M S}\right)$, where $\tau_{R M S}$ is the delay spread. In turn, the delay spread is given by $\tau_{R M S}=\sqrt{\left(1 / P_{t}\right) \sum P_{i} \tau_{i}^{2}-\tau_{0}^{2}}$ where $P_{t}$ is the total power in the channel and $P_{i}$ and $\tau_{i}$ are the power and delay of each tap, respectively.
} 


\section{CHAPTER 2. DIVERSITY TECHNIQUES}

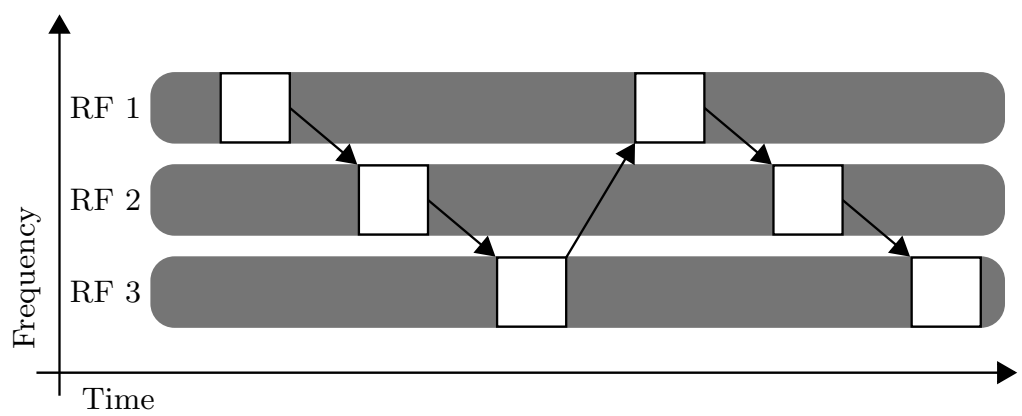

Figure 2.6: Time-Frequency Slicing.

two potential benefits: improved reception robustness due to better frequency diversity and increased capacity due to more efficient statistical multiplexing.

TFS improves frequency diversity by extending the frequency interleaving beyond one RF channel and across multiple RF channels. In this manner, it is possible to achieve better frequency diversity and compensate for the frequency dependency of the transmitter site, the receiver, and the propagation channel. The resulting gain is closely related to the number of $\mathrm{RF}$ channels that are bundled in the same TFS multiplex and their separation in the UHF band. In order to maximize the frequency diversity, the spacing between RF channels should be sufficient to cover the entire UHF band.

The capacity gain of TFS is related to the transmission of Variable Bit Rate (VBR) services and the utilization of StatMux [89]. VBR encoders generate a stream of variable bit rate so that the video quality remains constant. Traditionally, services are multiplexed according to their peak rate in order to ensure that enough capacity is always available. However, this means that some capacity will be wasted whenever the services are transmitted at a lower bit rate. StatMux relies on the fact that the aggregated peak rate of several VBR services is significantly lower than the peak rate of each individual service. The gain of StatMux depends on the number of channels that are bundled in the same multiplex. In particular, the gain increases with the number of services until reaching a certain saturation point. The utilization of TFS allows the bundling of several RF channels in order to create a high-capacity multiplex that can support a higher StatMux gain. This allows for an almost ideal StatMux gain with several HDTV services using VBR encoding. On the other hand, the gain is expected to be much lower with low bit rate services, as a single RF channel may already provide sufficient capacity to approach the saturation point of the StatMux gain. 
Although TFS was originally proposed in the standardization process of DVB-T2, it was finally only included in the standard as an informative annex due to the need of implementing a second tuner in receivers [4]. Reception of TFS services with a single tuner requires a time interval between the slots of the same service in order to perform frequency hopping. The minimum time that the receiver needs to switch to another $\mathrm{RF}$ channel and prepare for reception can be computed as $2 T_{C h E}+T_{u}$, where $T_{C h E}$ is the time required for channel estimation purposes and $T_{u}$ is the tuning time for switching from one $\mathrm{RF}$ channel to another. In the case of DVB-T2, the tuning time is generally estimated to be $5 \mathrm{~ms}$, while the time for channel estimation might be of several OFDM symbols depending on the pilot pattern. Due to the high rates of HDTV services, the time interval needed for frequency hopping cannot be guaranteed in DVB-T2, and a second tuner is required for TFS operation. In contrast, TFS has been adopted in DVB-NGH since the lower bit rates of mobile TV services allow receivers to perform frame hopping with a single tuner.

\subsection{Space Diversity Techniques}

\subsubsection{Single-Input Multiple-Output}

The utilization of multiple receive antennas, or SIMO, can provide not only diversity but also array gains. At the same time, it does not require any modification in standards or transmitters. Therefore, chip manufacturers can decide whether to implement multiple receive antennas in order to improve the performance of receivers at the expense of additional costs. Among the different mechanisms to combine the multiple received signals, such as Selection Combining (SC) or Equal Gain Combining (EGC), Maximal Ratio Receiver Combining (MRRC) is considered as optimum in the sense that it maximizes the resulting CNR and yields the best statistical reduction of fading [62]. Let us assume a SIMO receiver with two antennas. The signals corresponding to the first and second antenna $y_{1}$ and $y_{2}$ can be represented as:

$$
\begin{gathered}
y_{1}=h_{1} x+n_{1} \\
y_{2}=h_{2} x+n_{2},
\end{gathered}
$$

where $h_{1}, h_{2}$ are the complex channel realizations and $n_{1}, n_{2}$ are the complex Gaussian noise samples that correspond to the first and second antenna respectively. In this case, the receiver combining scheme for MRRC is as follows: 


$$
\begin{aligned}
\widetilde{x} & =h_{1}^{*} y_{1}+h_{2}^{*} y_{2} \\
& =\left(\left|h_{1}\right|^{2}+\left|h_{2}\right|^{2}\right) x+h_{1}^{*} n_{1}+h_{2}^{*} n_{2} \\
& =\|\left. h\right|^{2} x+h_{1}^{*} n_{1}+h_{2}^{*} n_{2},
\end{aligned}
$$

where $\widetilde{x}$ is the combined signal that results from MRRC.

\subsubsection{Multiple-Input Single-Output}

The utilization of multiple transmit antennas, or MISO, is motivated by the fact that it is generally more economical to add equipment to transmitters rather than to receivers, since one transmitter often serves hundreds to thousands of receivers [31]. Although MISO systems cannot achieve array gains in broadcasting systems by means of transmit beamforming techniques due to the lack of an uplink channel, they can still improve the performance as a result of better diversity.

\section{Alamouti Code}

The Alamouti code [31] is a very simple SFBC designed for increasing the diversity in systems with two transmit antennas. In OFDM systems, the Alamouti code operates by encoding in pairs the information symbols of adjacent subcarriers. If we assume that $x_{1}$ and $x_{2}$ are the information symbols to be transmitted in the first and second subcarrier respectively, the Alamouti code can be represented with the following transmission matrix:

$$
G_{\text {Alam }}=\left[\begin{array}{cc}
x_{1} & -x_{2}^{*} \\
x_{2} & x_{1}^{*}
\end{array}\right],
$$

where the rows correspond to transmit antennas and the columns correspond to subcarriers. From Eq. (2.12) we can see that the Alamouti code employs two subcarriers in order to transmit two information symbols, which results in a transmission rate of one symbol per subcarrier (rate 1).

The Alamouti code is well-known for achieving full diversity with reduced (linear) complexity required at the receiver side to demodulate the signal. The diversity order achieved by the Alamouti code in the i.i.d. Rayleigh MIMO channel is equal to $2 N_{r}$, where $N_{r}$ is the number of receive antennas. Regarding the decoding complexity, if the channel is assumed to be constant across the subcarriers of the Alamouti pair, a matched filter can be used to demodulate the receive signal in a similar manner to MRRC [31]. However, in real reception scenarios, the variation of the frequency response is likely to be significant and 
hence, the assumption of a constant channel response within pairs of adjacent subcarriers is not valid. In this case, different decoding algorithms with linear complexity such as ZF or MMSE can be used to recover the information symbols. In order to use the Alamouti code, it is necessary to employ alternate or orthogonal pilot patterns between antennas so that receivers can estimate the channel response from each transmit antenna. This also means that the number of pilots to be transmitted must be doubled for the same resolution of channel estimation in the time and frequency domains.

\section{Distributed Alamouti}

The implementation of a second antenna per transmitter for MISO purposes may be too costly for network operators, and it was the main reason why colocated MISO techniques based on multiple antennas per transmitter where not included in the DVB-T2 standard. Instead, a distributed MISO technique based on a modified version of the Alamouti code was incorporated [4]. In this case, the Alamouti code is performed across pairs of transmitters in order to improve the reception in $\mathrm{SFN}^{4}$. The arrival of similar-strength signals from different transmitters in LoS scenarios can cause deep notches in the frequency response of the channel. These notches can erase a significant percentage of subcarriers and degrade significantly the Quality of Service (QoS). By using the Alamouti code it is possible to combine the signals from different transmitters in an optimum way and remove the presence of notches from the channel.

If we assume that $x_{1}$ and $x_{2}$ are the information symbols to be transmitted in the first and second subcarrier respectively, the modified Alamouti code included in DVB-T2 can be represented with the following transmission matrix:

$$
G_{\text {AlamDis }}=\left[\begin{array}{cc}
x_{1} & x_{2} \\
-x_{2}^{*} & x_{1}^{*}
\end{array}\right],
$$

where the rows correspond to transmitters and the columns correspond to subcarriers. From Eq. (2.13), we can see that one transmitter sends the information symbols without any modification, while the other transmitter sends an encoded version of the original symbols. The modified version of the Alamouti code included in DVB-T2 possesses the same properties of the original code, i.e. full diversity with reduced complexity.

\footnotetext{
${ }^{4} \mathrm{SFN}$ in OFDM systems are constituted by multiple transmitters that operate simultaneously at the same RF channels. The guard interval included in the OFDM symbols allows the signals coming from the different transmitters to be combined in a constructive manner [21]. SFN allow for a simpler network planning and generally achieve a higher spectral efficiency in terrestrial scenarios.
} 


\section{CHAPTER 2. DIVERSITY TECHNIQUES}

\section{Delay Diversity}

DD belongs to a family of techniques that transform space diversity into time or frequency diversity. The main advantage of these techniques lies in the fact that they do not require specific pilot patterns or signal processing to demodulate the signal and hence, they can be employed without any modification in standards or receivers [33]. Other techniques from the same family are CDD, PD and SD. Although the utilization of these techniques does not result in an optimal averaging of fading, like Alamouti, they can achieve greater diversity than single transmit antenna configurations. In particular, DD consists in applying a delay in the order of microseconds to the signal from the second antenna. The combination of the signals from both antennas results in a single transmit antenna channel with increased frequency diversity that is exploited by the FEC code. According to previous investigations, delay values around $1.5 \mu \mathrm{s}$ are sufficient for increasing the frequency diversity in DD systems [34, 54]. It must be noted that DD is not well suited for distributed MISO, since it cannot erase the fades caused by LoS reception in SFN.

\section{eSFN}

A novel transmit diversity scheme known as enhanced Single Frequency Network (eSFN) [90] has been adopted in DVB-NGH. The main idea of eSFN is to apply a linear predistortion function to each antenna in such a way that it does not affect the channel estimation in receivers. As with DD, this increases the frequency diversity of the channel without the need of specific pilot patterns or signal processing to demodulate the signal. eSFN is also well suited for its utilization as a distributed MISO technique in SFN. The randomization performed in each transmitter can avoid the negative effects caused by LoS components in this kind of networks. In addition, by using a different predistortion function at each transmitter, it is possible to allow for unique transmitter identification within the network, which can be used e.g. for monitoring applications.

\subsubsection{Multiple-Input Multiple-Output}

The utilization of multiple antennas at the transmitter and receiver side, or MIMO, allows the multiplexing rate of information across the antennas to be increased. This can result in a significant improvement of the system performance, especially at high CNR values [32]. The main drawback lies in the mandatory implementation of multiple antennas at receivers, which may be too costly from the chip manufacturer's point of view. It should be pointed out that, although transmit diversity techniques designed for MISO systems can 
generally be combined with multiple receive antennas for higher diversity, they do not provide multiplexing gains.

\section{Spatial Multiplexing}

The most simple way of increasing the data rate consists in dividing the information symbols between the transmit antennas. This is referred to as pure Spatial Multiplexing (SM) or simply SM. In the case of two transmit and two receive antennas, it can be represented with the following transmission matrix:

$$
G_{S M}=\left[\begin{array}{l}
x_{1} \\
x_{2}
\end{array}\right],
$$

where the rows correspond to transmit antennas and the columns correspond to subcarriers. From Eq. (2.14) we can see that SM employs a single subcarrier to transmit two information symbols, which results in a multiplexing rate of two symbols per subcarrier (rate 2).

Due to the fact that each information symbol is transmitted across a single antenna, SM provides no diversity at the transmitter side. In particular, the diversity order achieved by spatial multiplexing in uncoded systems is equal to the number of received antennas. Nevertheless, an outer FEC code can still exploit the diversity at the transmitter side if the information symbols that are transmitted across different antennas belong to the same codewords [91].

\section{Enhanced Spatial Multiplexing}

The presence of correlation in the MIMO channel due to the lack of scattering or insufficient antenna separation is specially detrimental for SM [92]. A novel scheme proposed in DVB-NGH known as enhanced Spatial Multiplexing (eSM) retains the multiplexing capabilities of SM, and at the same time, increases the robustness against spatial correlation. In order to do so, the information symbols are weighted and combined before their transmission across the antennas. In addition, a phase hopping term is added to the second antenna in order to randomize the code structure and avoid the negative effect of certain channel realizations. The transmission matrix of eSM can be represented as the concatenation of the regular SM transmission matrix with a precoding matrix:

$$
G_{e S M}=\left[\begin{array}{cc}
\cos \phi_{p} & \sin \phi_{p} \\
e^{j \phi_{t}(n)} \sin \phi_{p} & -e^{j \phi_{t}(n)} \cos \phi_{p}
\end{array}\right]\left[\begin{array}{l}
x_{1} \\
x_{2}
\end{array}\right]
$$

From Eq. (2.15) we can see that the multiplexing rate of SM is maintained, since two information symbols are transmitted in a single subcarrier. The weighting of the information symbols depends on the rotation angle $\phi_{p}$, which 


\section{CHAPTER 2. DIVERSITY TECHNIQUES}

has been specifically tuned for each constellation. On the other hand, the phase hopping term $e^{j \phi_{t}(n)}$ included in the precoding matrix changes periodically within each codeword. In this way it is possible to average the negative effects of channel realizations that are detrimental to the code structure.

\subsection{Conclusions}

The utilization of TI presents a series of trade-offs regarding the zapping time, the power saving and the memory requirements. The zapping time is generally proportional to the interleaving duration, and therefore, interleaving durations up to several seconds are not practical unless fast zapping techniques can reduce the zapping time down to tolerable values. Power saving is achieved in mobile broadcasting systems by transmitting the information in a discontinuous manner over time. However, this might reduce the reception robustness with TI as a result of worse time diversity. Lastly, the amount of memory that is required for time deinterleaving purposes depends on whether the TI and the FEC is performed at the physical or upper layers. PL-FEC mechanisms generally achieve better performance due to the utilization of soft decision decoding at a bit level, whereas UL-FEC mechanisms employ erasure decoding at a packet level. Although the use of packet erasure decoding reduces the error correction capabilities of UL-FEC, it supports the provision of long TI with much lower memory requirements. The performance loss of UL-FEC compared to PL-FEC is highest in stationary channels and lowest in mobile channels with an on-off behaviour.

Several UL-FEC mechanisms are available in the DVB family of standards for the protection of streaming services. This is the case of the MPE-FEC and MPE-iFEC techniques developed during the standardization processes of DVB-H and DVB-SH, respectively. MPE-FEC is designed to counter fast fading with interleaving durations in the order of hundreds of milliseconds. In contrast, MPE-iFEC can compensate for the presence of shadowing in the received signal by using interleaving durations up to several seconds. Besides MPE-FEC and MPE-iFEC, AL-FEC, which was standardized for the protection of file delivery services in DVB-H, can be adapted for streaming services with some minor modifications. On the other hand, PL-FEC mechanisms with time interleaving have been included in DVB-T2, DVB-SH and DVB-NGH. In DVB-T2, time diversity is achieved by means of BI, resulting in zapping times around one and a half times the interleaving duration. In contrast, DVB-SH and DVB-NGH employ a combination of BI and CI to obtain time diversity. The utilization of CI reduces the end-to-end delay and the zapping time, and with UL configurations, it can provide long TI with tolerable zapping times. 
In the frequency domain, the utilization of COFDM and frequency interleaving generally achieves sufficient frequency diversity in the $8 \mathrm{MHz}$ channels of the UHF band. In spite of this, large signals variations can be encountered between different RF channels due to the frequency dependency of the transmitter site, the receivers and the propagation path. TFS improves the diversity in the frequency domain by extending the interleaving across multiple RF channels so that the FEC decoder can average the signal variations between channels. The utilization of TFS might also increase the system capacity as a result of more efficient StatMux.

In addition to FEC and interleaving, it is possible to exploit the diversity in the time and frequency domains by means of rotated constellations and component interleaving. The first spreads the binary information across a higher number of components in the information symbols, whereas the second ensures that each component experiences independent fading. The improvement of rotated constellations comes at the expense of higher demodulation complexity, as all the components of the information symbols must be demodulated together in order to retrieve the binary information.

Regarding the utilization of multiple antennas, it can be categorized in SIMO, MISO and MIMO depending on whether multiple antennas are used at the receiver side, at the transmitter side, or at both sides. SIMO systems can achieve diversity and array gains without the need of any modification in standards or transmitters. In addition, the utilization of SIMO techniques does not require the transmission of a higher number of pilots and can be solved with linear demodulation complexity in receivers by means of MRRC. MISO systems cannot provide array gains in mobile broadcasting systems due to the lack of an uplink channel, and they only improve the performance by means of diversity. The Alamouti code is a transmit diversity technique designed for two transmit antennas that achieves full diversity with linear decoding complexity. However, it doubles the amount of pilots that is needed for channel estimation and requires the implementation of specific signal processing in receivers. Alternatively, schemes such as DD or eSFN have been proposed in order to achieve spatial diversity without the need of additional pilots or any modification in the operation of receivers. The main disadvantage of traditional MISO techniques lies in the implementation of multiple antennas per transmitter, which might be too costly for network operators. Because of this, a distributed MISO technique based on the Alamouti code that is performed across different transmitters was included in DVB-T2.

Compared to SIMO and MISO, the most important feature of MIMO systems is that they can provide multiplexing gains by increasing the transmission rate of information across the antennas. The most simple way of increasing the multiplexing rate of information consists in simply dividing the information 
symbols between the transmit antennas, which is referred to as spatial multiplexing. An enhanced version of spatial multiplexing designed for improved robustness in the presence of correlation have been adopted in DVB-NGH. 


\section{Chapter 3}

\section{Information-Theoretic Investigation of Diversity Techniques}

In this chapter, we describe the use of diversity from an information-theoretic point of view. To this purpose, we evaluate the performance of diversity techniques in mobile broadcasting systems based on the formulas that characterize the outage capacity of the channel. The outage capacity formulation used in this chapter was proposed in [61] for fading channels and applied to MIMO channels in [32]. We provide specific values that illustrate the gain obtained in each domain, i.e. time, frequency and space, as well as the mutual influence between different types of diversity. Although these results can be considered as upper bounds of the gains that can be achieved in real systems, they give a meaningful insight into the performance of diversity techniques. Moreover, we evaluate the gain of rotated constellations in mobile broadcasting systems by computing the BICM capacity [64] in fading channels.

The rest of the chapter is organized as follows. First, in Section 3.1, we introduce the formulas that characterize the outage channel capacity. Then, in Section 3.2, we describe the methodology used to model the performance of mobile broadcasting systems, and present the information-theoretic results showing the fading margin gains of diversity techniques. In Section 3.3, we focus on rotated constellations. Finally, we deliver some concluding remarks in Section 3.4. 


\subsection{Capacity of Fading Channels}

\subsubsection{Outage Capacity of Fading Channels}

From now on we will assume that the channel is unknown to the transmitter, which is generally the case of broadcasting systems due to the lack of an uplink channel. In addition, we will assume full knowledge of the channel at the receiver side, which is consistent with the ideal channel estimation that we consider in the rest of the thesis. With these considerations in mind, the capacity of the fading channel, $C$, can be expressed as:

$$
C=\log _{2}\left(1+|h|^{2} \Gamma\right) \text { bps } / \mathrm{Hz} .
$$

Eq. (3.1) measures the maximum rate of information at which error-free communication is possible as a function of the CNR, here denoted as $\Gamma$, and the channel gain $|h|$. In the case of multipath fading, $|h|$ is usually modelled as a Rayleigh distributed random variable. Strictly speaking, the presence of fading in the received signal reduces the channel capacity to zero, since error-free communication is no longer possible whenever $|h|$ causes the channel capacity to drop below the rate of information. In this case, the system is said to be in outage. The capacity of fading channels is generally expressed in outage terms as the maximum rate of information that can be achieved so that a target outage probability is not exceeded [61]. The outage probability of the channel, $p(R)$, is given by:

$$
p(R)=\operatorname{Pr}\left\{\log _{2}\left(1+|h|^{2} \Gamma\right)<R\right\},
$$

where $R$ is the rate of information. The corresponding outage capacity, $C_{\epsilon}$, is:

$$
C_{\epsilon}=\max \{R \mid p(R) \leq \epsilon\} \mathrm{bps} / \mathrm{Hz},
$$

where $\epsilon$ is the target outage probability.

\subsubsection{FEC and Interleaving}

In order to account for the utilization of FEC and interleaving, the codewords can be assumed to be transmitted on a parallel channel consisting in a set of non-interfering sub-channels, each of which is corrupted by independent noise and affected by a particular channel realization. Each channel realization corresponds to a different OFDM symbol in the time domain and to a different subcarrier in the frequency domain. If the codewords are transmitted across $L$ channel realizations, an outage will occur whenever the average capacity of the 
channel across the codeword drops below the rate of information. The outage probability of a parallel channel is:

$$
p(R)=\operatorname{Pr}\left\{\frac{1}{L} \sum_{l=1}^{L} C_{l}<R\right\},
$$

where $C_{l}$ corresponds to the capacity of channel realization $l$. If enough channel realizations are averaged, as $L \rightarrow \infty$, the law of large numbers states that the channel capacity approaches the expected value:

$$
C_{\text {erg }}=\mathrm{E}\left[C_{l}\right] \mathrm{bps} / \mathrm{Hz} .
$$

Therefore, by coding across a sufficient number of channel realizations it is possible to remove the uncertainty caused by fading and approach the ergodic capacity of the fading channel. It must be noted that the ergodic capacity of the fading channel is always lower than the capacity of the AWGN channel. While the loss is negligible in the low CNR region $(<0 \mathrm{~dB})$, there is a constant difference at high $\mathrm{CNR}$ values. In the case of the Rayleigh channel, the difference is approximately equal to $2.5 \mathrm{~dB}$ for CNRs higher than $10 \mathrm{~dB}$ [24].

\subsubsection{MIMO}

MIMO techniques can be used to achieve diversity and minimize the fluctuations caused by fading. Depending on whether the antennas are implemented in the receiver side, the transmitter side, or in both sides, we talk about SIMO, MISO or MIMO systems. We also distinguish between MIMO systems that sacrifice multiplexing rate for diversity, and MIMO systems that exploit the diversity and multiplexing capabilities of the MIMO channel in an optimal manner [93]. We refer to the first class as diversity MIMO (full diversity) whereas we use the term optimal MIMO (full rate and full diversity) for the second class.

The capacity of the SIMO channel, $C_{\text {SIMO }}$, is:

$$
C_{\text {SIMO }}=\log _{2}\left(1+\|\mathbf{h}\|^{2} \Gamma\right) \text { bps } / \mathrm{Hz},
$$

where $\mathbf{h}=\left[h_{1}, h_{2}, \ldots, h_{M_{r}}\right]$ is the vector containing the channel gains corresponding to each receive antenna, and $M_{r}$ is the number of receive antennas. From Eq. (3.6), we can see that the use of SIMO not only results in a diversity gain due to averaging of fading across antennas, but also in an array gain due to the coherent combination of signals.

If we assume a total power constraint across the transmit antennas, i.e. the total transmitted power remains the same as with a single antenna, the capacity of the MISO channel, $C_{\mathrm{MISO}}$, is: 


$$
C_{\mathrm{MISO}}=\log _{2}\left(1+\|\mathbf{h}\|^{2} \frac{\Gamma}{M_{t}}\right) \mathrm{bps} / \mathrm{Hz}
$$

where $\mathbf{h}=\left[h_{1}, h_{2}, \ldots, h_{M_{t}}\right]$ is the vector containing the channel gains corresponding to each transmit antenna, and $M_{t}$ is the number of transmit antennas. From Eq. (3.7) we can see that although the use of MISO achieves a diversity gain due to the averaging of fading across antennas, it does not provide an array gain. Since the transmitter does not possess channel knowledge, the signals cannot be transmitted with the correct phase so that they can be added coherently at the receiver side (i.e. transmit beamforming). As a result, there is no increase in the total received CNR.

Assuming the same total power constraint across the transmit antennas as with MISO, the capacity of diversity MIMO, $C_{\text {divmimo, }}$ can be computed as:

$$
C_{\text {divMimo }}=\log _{2} \operatorname{det}\left(1+\frac{\Gamma}{M_{t}} \operatorname{Tr}\left\{\mathbf{H H}^{*}\right\}\right) \mathrm{bps} / \mathrm{Hz},
$$

where $\operatorname{det}()$ is the determinant operator, $\operatorname{Tr}()$ is the trace operator, and $\mathbf{H}$ is the $M_{r} \times M_{t}$ MIMO channel matrix. On the other hand, the capacity of optimal MIMO, $C_{\text {optMimo, is: }}$

$$
C_{\text {optMIMO }}=\log _{2} \operatorname{det}\left(\mathbf{I}_{M_{r}}+\frac{\Gamma}{M_{t}} \mathbf{H H}^{*}\right) \mathrm{bps} / \mathrm{Hz},
$$

where $\mathbf{I}_{M_{r}}$ is the $M_{r} \times M_{r}$ identity matrix.

Fig. 3.1 illustrates the outage and ergodic capacity of the i.i.d. Rayleigh channel, in which the entries of $\mathbf{H}$ are assumed to be independent, identically distributed, and circular symmetric complex Gaussian variables. It should be pointed out that SIMO and MISO perform identically for both the outage and the ergodic case, except for the $3 \mathrm{~dB}$ advantage of SIMO due to the array gain. As it is shown in the figure, the utilization of SIMO, MISO and diversity MIMO results in an offset of the capacity curves that does not affect its slope. From the figure we can also notice that the improvement of these techniques is very much reduced in the ergodic case. In other words, if sufficient diversity is already achieved in the time and frequency domain, the benefits of additional diversity in the space domain become negligible. The reason for this is that the higher the diversity, the lower the benefits that are obtained with additional diversity. This is usually referred to as the diminishing marginal returns of diversity. In contrast, the multiplexing gain achieved by optimal MIMO modifies the rate of growth of the capacity with the CNR. While the improvement over diversity MIMO is limited at low CNR values, it becomes more significant as the CNR increases. 

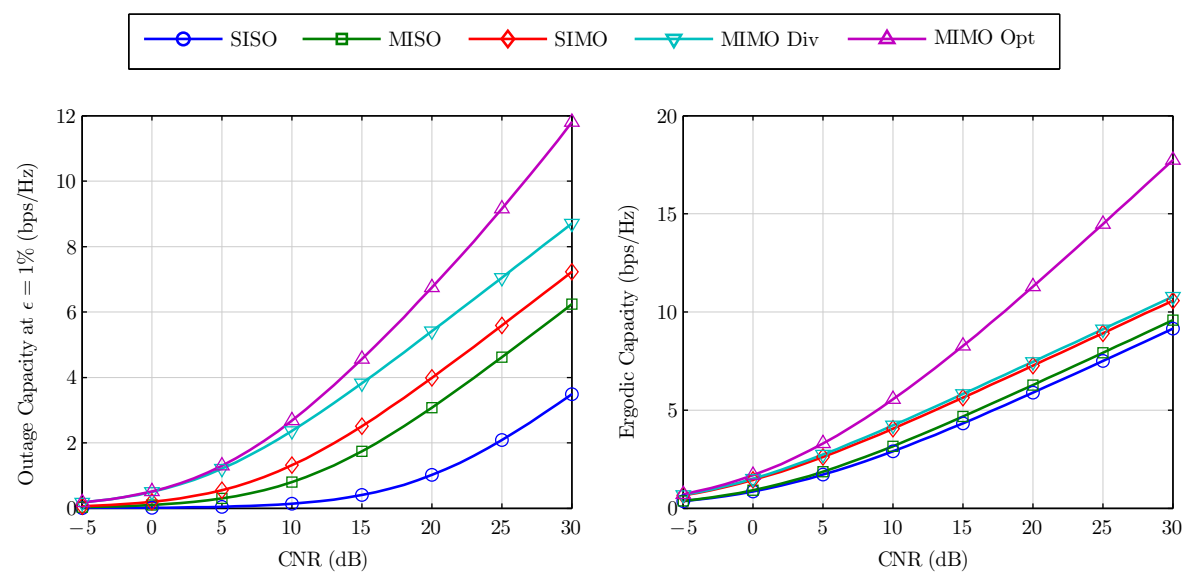

Figure 3.1: Capacity of the i.i.d. Rayleigh channel. Outage capacity for a target outage probability of $1 \%$ on the left, and ergodic capacity on the right.

\subsection{Fading Margin Gains of Time, Frequency and Space Diversity in Mobile Broadcasting Systems}

\subsubsection{Definition of Fading Margin}

Mobile broadcasting networks are planned so that a certain QoS criterion, generally expressed in terms of error probability, is satisfied in the entire coverage area. In order to compensate for fading and ensure that reliable communication is maintained at the target QoS, it is necessary to add an extra fading margin of several dBs to the link budget [37]. Diversity techniques such as FEC and interleaving, or MIMO, are aimed to reduce the fading margin for any given QoS criterion and approach the capacity of the AWGN channel. In this thesis, we define the fading margin gain as the reduction in fading margin that can be achieved with diversity techniques. For the purpose of computing the fading margin gain, we also define the outage $\mathrm{CNR}, \Gamma_{\epsilon}(R)$, as the minimum $\mathrm{CNR}$ that is required at a transmission rate, $R$, so that a target outage probability, $\epsilon$, is not exceeded:

$$
\Gamma_{\epsilon}(R)=\min \{\Gamma \mid p(R) \leq \epsilon\} \text { bps } / \mathrm{Hz} .
$$

After this, the fading margin can be easily computed as: 


\section{CHAPTER 3. INFORMATION-THEORETIC INVESTIGATION OF DIVERSITY TECHNIQUES}

$$
\text { Fading Margin }=\Gamma_{\epsilon}(R)-\Gamma_{\text {AWGN }}(R),
$$

where $\Gamma_{\text {AWGN }}(R)$ is the CNR value for which the capacity of the AWGN channel equals $R$. In the case of Rayleigh fading, the fading margin is $10 \mathrm{~dB}$ for $\epsilon=10 \%$, $20 \mathrm{~dB}$ for $\epsilon=1 \%$, and $30 \mathrm{~dB}$ for $\epsilon=0.1 \%$. Since the fading margin increases as the target outage probability goes down, the potential gain of diversity techniques grows when a more strict QoS criterion is used.

\subsubsection{Modelling of Mobile Broadcasting Systems}

In order to investigate the fading margin gains that can be achieved in mobile broadcasting systems by means of diversity, we consider a system in which the codewords are mapped on all the subcarriers in a given bandwidth, and across a number of OFDM symbols that is given by the interleaving duration [94]. The capacity of such a system, $C_{\text {broad }}$ can be modelled as:

$$
C_{\text {broad }}=\frac{1}{L} \frac{1}{K} \sum_{l=1}^{L} \sum_{k=1}^{K} C_{l k} \text { bps } / \mathrm{Hz},
$$

where $K$ is the total number of subcarriers covered by the transmission bandwidth, $L$ is the total number of OFDM symbols covered by the interleaving duration, and $C_{l k}$ is the channel capacity that correspond to the subcarrier $k$ of the OFDM symbol $l$. Depending on whether SISO, SIMO, MISO, diversity MIMO, or optimal MIMO are used, $C_{l k}$ will correspond to the formulas in Eq. $(3.1,3.6,3.7,3.8,3.9)$ respectively.

It should be noted that since Eq. (3.12) is based on Shannon limit, the resulting capacity is not conditioned by particular aspects of BICM systems such as the bit labelling, the constellation or the code rate [64]. It is also important to notice that Eq. (3.12) represents the best case scenario for diversity, since the codewords are assumed to be spread across all the subcarriers within the transmission bandwidth, and across all the OFDM symbols within the interleaving duration. Due to the fact that in real broadcasting systems each codeword is only transmitted in a subset of subcarriers and/or OFDM symbols, the values obtained with this model may be considered as upper bounds of the gains that can be achieved in real systems.

\subsubsection{Performance Evaluation}

For the investigation on TI it is important to distinguish between fast fading and shadowing (see Section 2.4). The first is caused by multipath propagation and relates to displacements in the scale of one wavelength. The second is 
originated from the presence of obstacles such as building and trees and might extend across hundreds of meters. In order to account for a frequency selective fast fading scenario, we have selected the Typical Urban 6 (TU6) channel model [95], which is representative of vehicular reception for Doppler frequencies above $10 \mathrm{~Hz}$ [96]. The channel is made of 6 taps having wide dispersion in delay and relatively strong power. The taps are defined as pure non Line-ofSight $(\mathrm{nLoS})$ in the sense that a LoS component is not included. For MIMO systems with co-polarized antennas, we have modelled each propagation path between the transmit and the receive antennas as an independent TU6 profile, so that no spatial correlation exists between antennas. This is generally the case of vehicular receivers, in which the separation between receive antennas can be sufficiently large to ensure independent fading at UHF frequencies.

In contrast with vehicular receivers, cross-polarized antennas are usually preferable in handheld receivers due to the limited separation between antennas that can be achieved in this kind of devices. In this case, we have selected the outdoor mobile channel developed during the standardization process of DVB-NGH [97]. The channel is made of 8 taps with different values of delay and power gain, and includes an XPD factor describing the energy coupling between cross-polarized paths, a K factor describing the power ratio between LoS and $n L o S$ components, and a covariance matrix describing the spatial correlation between antennas. Additional terms for antenna rotation and asymmetry are also included. In particular, a rotation matrix describes the presence of polarization mismatch between the transmit and the receive antennas, whereas an asymmetry matrix describes the presence of power imbalance between polarizations.

The TU6 and NGH outdoor channels describe fast fading as seen during antennas displacements of up to $50 \mathrm{~m}$. Therefore, it is necessary to include the presence of shadowing in order to evaluate the gains of TI for interleaving durations higher than 1 s. In particular, we have assumed a user moving at constant velocity across a log-normal CNR map, which is defined by its standard deviation $(\sigma)$ and its correlation distance $\left(d_{\text {corr }}\right)$ as described in [19]. The shadowing model outputs CNR values that correspond to the shadowing experimented by the signal every $100 \mathrm{~ms}$. In this case, the model has been configured with $\sigma=5.5 \mathrm{~dB}$ and $d_{\text {corr }}=20 \mathrm{~m}$.

In addition to terrestrial channels, we also consider the reception in mobile satellite scenarios by using the statistical model for LMS presented in [98]. This model incorporates the presence of different degrees of shadowing with a Markov chain constituted by three states: LoS, moderate shadowing, and deep shadowing. The model combines fast fading and shadowing by means of a Loo distribution with different parameters for each Markov state. The model is parameterized for different velocities $v$, elevation angles $\theta$, frequency bands, 


\section{CHAPTER 3. INFORMATION-THEORETIC INVESTIGATION OF DIVERSITY TECHNIQUES}

and reception environments. For our calculations, we have selected $v=60$ $\mathrm{km} / \mathrm{h}, \theta=40$ degrees, $f_{\mathrm{rf}}=2.2 \mathrm{GHz}$ (S-band) in the Suburban (SU) and Intermediate Tree Shadowed (ITS) environments. These are the parameters used in the standardization process of DVB-NGH for the evaluation of hybrid terrestrial-satellite profiles.

Unless stated otherwise, the results have been obtained for a target outage probability of $1 \%$ and a rate of information of $2 \mathrm{bps} / \mathrm{Hz}$. Also, the signal bandwidth is considered to be $8 \mathrm{MHz}$, which is the typical value of terrestrial UHF channels in Europe. In order to obtain statistically consistent results, the values presented in this chapter correspond to channel processes of several hours, which would be difficult to obtain with physical layer simulations due to the required computational time.

\subsubsection{Outage Capacity Results}

\section{Time Diversity}

In the case of time diversity, it is important to note that interleaving durations in the order of hundreds of milliseconds improve the reception robustness against fast fading, whereas interleaving durations in the order of seconds can also compensate for the presence of shadowing in the received signal. Consequently, we distinguish in Fig. 3.2 between the gains of TI corresponding to interleaving durations lower than $1 \mathrm{~s}$ in fast fading channels (left), and the gains of TI corresponding to interleaving durations up to several seconds in channels that include fast fading and shadowing (right). In the figure, the fading margin gains of TI are represented according to the number of coherence periods that are covered by the interleaving duration. This allows us to assess the potential gain of TI regardless of the user velocity.

The results in Fig. 3.2 show that TI can provide gains up to $5 \mathrm{~dB}$ in the TU6 channel, although the gain is much lower in the NGH outdoor channel. The reason is that the NGH outdoor channel includes a LoS component that reduces the impact of fast fading in the received signal and diminishes the benefit of using TI. On the other hand, the gain of TI is significantly higher in LMS scenarios, as in this case, interleaving durations up to several seconds can provide further gains against shadowing. It is important to note that although the largest gain is achieved in the LMS channels, gains up to $12 \mathrm{~dB}$ are possible in the terrestrial scenario (TU6 channel with shadowing). In all channels, the gain of TI begins to saturate when the interleaving duration covers approximately fifty coherence times. In the case of fast fading with Doppler $33.3 \mathrm{~Hz}(60 \mathrm{~km} / \mathrm{h}$ at $600 \mathrm{MHz})$, this corresponds to approximately $200 \mathrm{~ms}$ of TI. In the case of shadowing with $d_{\text {corr }}=20 \mathrm{~m}$ and $v=60 \mathrm{~km} / \mathrm{h}$, 


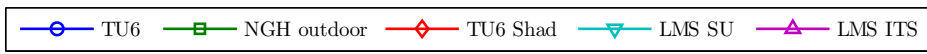
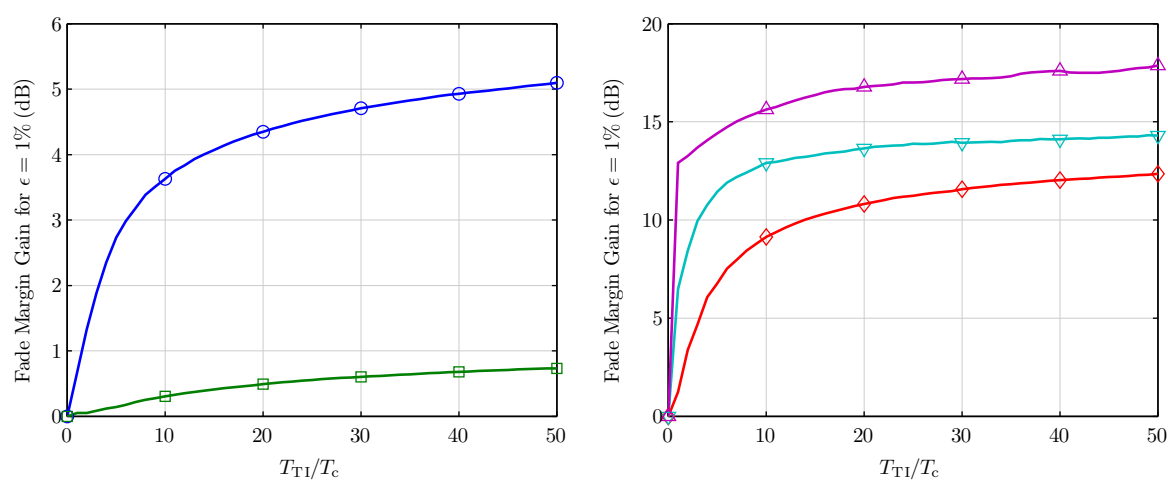

Figure 3.2: Fading margin gain of TI against fast fading on the left, and against fast fading with shadowing on the right.

fifty coherence times correspond to approximately $60 \mathrm{~s}$ of TI, which is generally not practical in mobile broadcasting systems. For a more realistic interleaving duration, such as $10 \mathrm{~s}$, the gain of TI is around $8 \mathrm{~dB}$ in the terrestrial scenario, and up to $15 \mathrm{~dB}$ in the LMS channels.

\section{Frequency Diversity}

Frequency interleaving in mobile broadcasting systems is generally performed within a single RF channel in the UHF band, which limits the frequency diversity to less than $8 \mathrm{MHz}$. TFS extends the frequency interleaving beyond one $\mathrm{RF}$ channel and across hundreds of $\mathrm{MHz}$ by bundling several $\mathrm{RF}$ channels into the same multiplex (see Section 2.5.2).

In order to evaluate the fading margin gains of TFS, it is necessary to model the signal variations between different channels in the UHF band. In this dissertation, we assume that there is no correlation of fast fading between RF channels, whereas there is a certain correlation of shadowing. The assumption of uncorrelated fast fading is generally safe, as the channel separation should be much larger than the coherence bandwidth of multipath propagation scenarios. On the other hand, the correlation of shadowing in the frequency domain depends on the separation between any pair of RF channels in the TFS multiplex. The larger the separation, the lower the correlation of shadowing between RF channels. In addition to fast fading and shadowing, we consider the presence of a power imbalance caused, among other factors, by the frequency depen- 

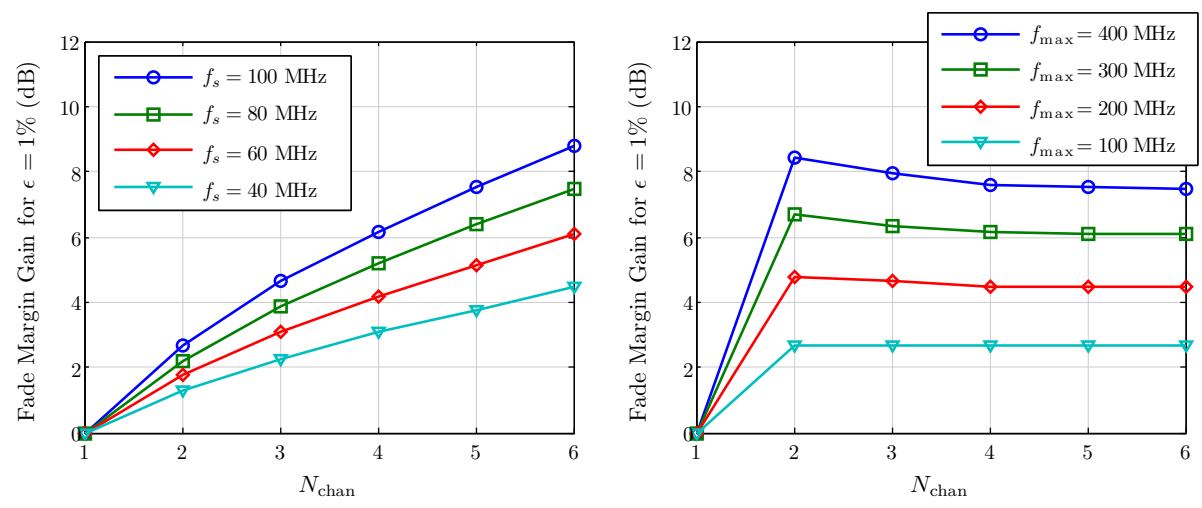

Figure 3.3: Fading margin gain of TFS according to the number of RF channels per multiplex in the TU6 channel with shadowing. The frequency separation between consecutive channels is $f_{s}$ (left), and the maximum separation between the lowest and the highest channel is $f_{\max }$ (right).

dency of the antenna aperture and the height loss [99]. The power imbalance has been modelled as an offset of the mean CNR that is proportional to the nominal frequency of each RF channel. The correlation of shadowing in the frequency domain and the power imbalance between RF channels have been parameterized according to the model presented in [100]. This is based on field measurements performed by the Swedish DTT operator, Teracom.

Fig. 3.3 illustrates the fading margin gains of TFS according to the number of RF channels that are grouped with TFS in the same multiplex. In the left side of the figure, the RF channels begin in $470 \mathrm{MHz}$ and move upwards in frequency according to the channel separation, $f_{s}$. For example, the RF channels grouped in the TFS multiplex for $f_{s}=100 \mathrm{MHz}$ are those located in $470 \mathrm{MHz}, 570 \mathrm{MHz}, 670 \mathrm{MHz}$ and so on. In this case, the gain of TFS grows larger when more channels are grouped in the TFS multiplex, and when the separation between them increases. This results in a higher portion on the UHF band being covered by the frequency interleaving, and therefore, it achieves better diversity in the frequency domain. It should be noted, however, that some configurations shown in Fig. 3.3 are not practical since they exceed the bandwidth used for DTT services in the UHF band $(\leq 400 \mathrm{MHz})$. For a realistic TFS scenario using four RF channels and $f_{s}=100 \mathrm{MHz}$ (i.e. $470 \mathrm{MHz}$, $570 \mathrm{MHz}, 670 \mathrm{Mhz}$ and $770 \mathrm{MHz}$ ), the gain of TFS is around $6 \mathrm{~dB}$.

In the right side of Fig. 3.3, the RF channels are uniformly spaced in the UHF band according to the maximum separation in the multiplex, $f_{\max }$. For example, with $f_{\max }=300 \mathrm{MHz}$, the RF channels grouped in the TFS multiplex 

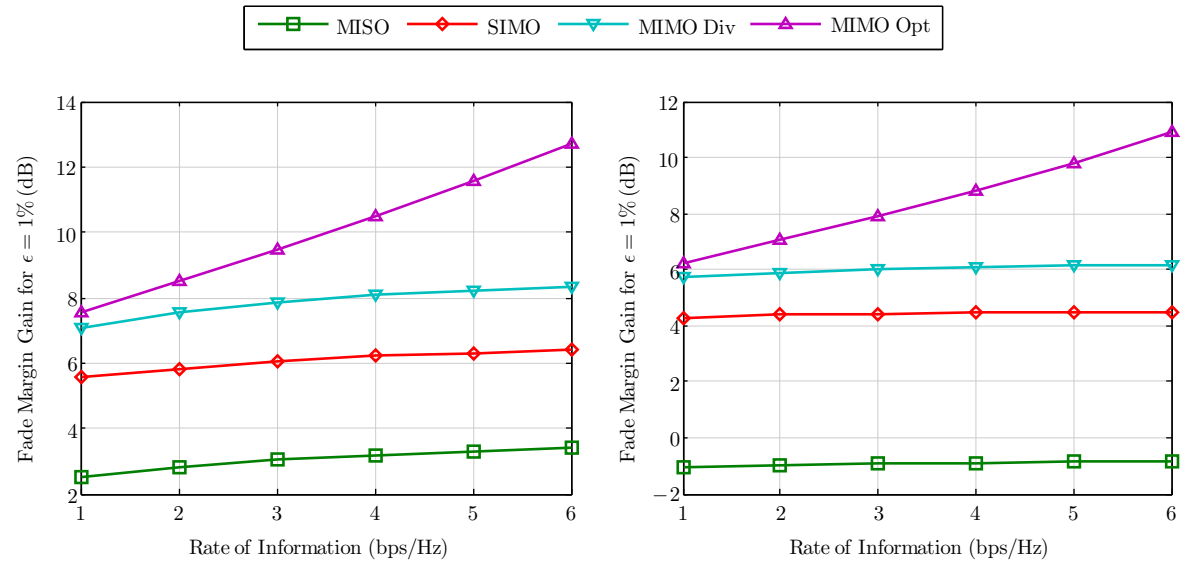

Figure 3.4: Fading margin gain of MIMO with co-polarized antennas in the TU6 channel (left), and with cross-polarized antennas in the NGH outdoor channel (right).

are uniformly spaced between $470 \mathrm{MHz}$ and $770 \mathrm{MHz}$. In this case, the gain of TFS saturates with just two RF channels. A higher number of channels does not improve the performance, and depending on the maximum separation in the multiplex, it can even reduce the TFS gain. These results reveal that the fading margin gain of TFS depends mostly on the extent of interleaving in the frequency domain, and that the number of $\mathrm{RF}$ channels in the multiplex does not affect the performance in a significant manner.

\section{Space Diversity}

Fig. 3.4 shows the fading margin gains of MIMO systems with co-polarized antennas (vehicular) and cross-polarized antennas (handheld) according to the outage capacity of the TU6 and the NGH outdoor channels, respectively. In the case of co-polarized antennas, all the MIMO configurations improve the system performance compared to SISO. It should be noted, however, that the gain of MISO is much lower than the rest of configurations, as it lacks the $3 \mathrm{~dB}$ array gain that is achieved with a pair of receive antennas. In this sense, it is important to distinguish between the diversity gain that results from better protection against multipath fading, and the multiplexing gain that is achieved by increasing the transmission rate of information symbols across the antennas. The first does not depends on the CNR and can be seen as an offset in the outage capacity of the channel, whereas the second modifies the rate of growth of the capacity with the CNR (see Fig. 3.1). Because of this, the gain 

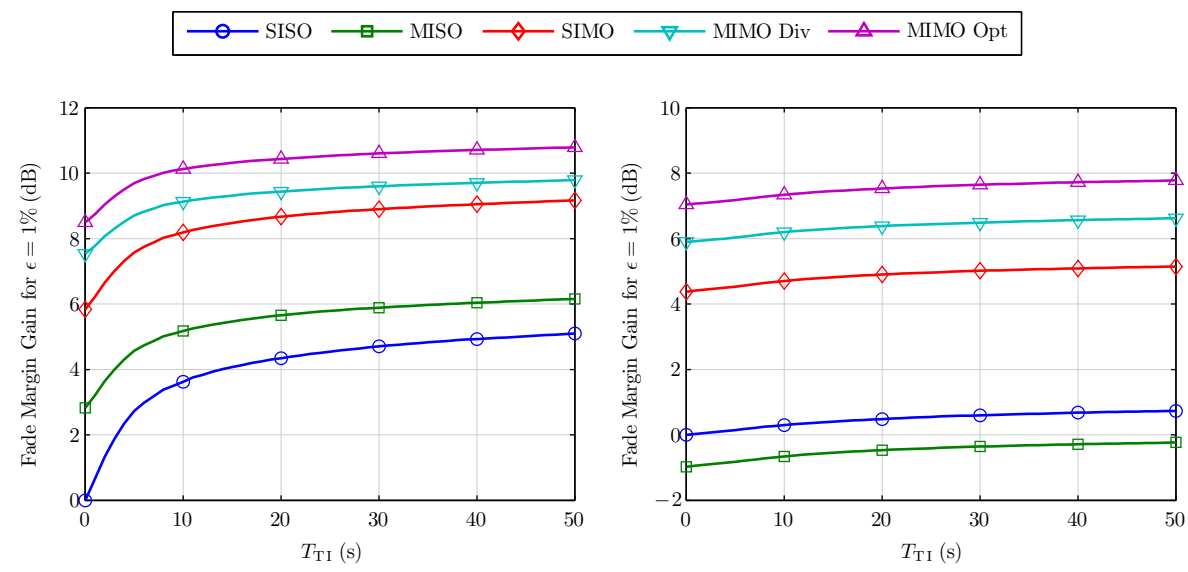

Figure 3.5: Combined gain of TI and MIMO with co-polarized antennas in the TU6 channel (left), and with cross-polarized antennas in the NGH outdoor channel (right).

of SIMO, MISO and diversity MIMO remains constant, whereas the gain of optimal MIMO grows linearly with the rate of information. With $1 \mathrm{bps} / \mathrm{Hz}$, which is a common transmission rate for mobile services (i.e. QPSK code rate $1 / 2$ ), optimal MIMO provides a similar gain as diversity MIMO. With $4 \mathrm{bps} / \mathrm{Hz}$, which is more typical of fixed transmissions (e.g 64QAM code rate 2/3), optimal MIMO outperforms diversity MIMO in about $3 \mathrm{~dB}$.

Compared to the case with co-polarized antennas, the fading margin gains of MIMO systems with cross-polarized antennas are lower, but still significant. Cross-polarized antennas provide better robustness against polarization effects, even if the presence of correlation between antenna paths reduces the diversity gain against fading. The only exception to this is MISO, for which the use of multiple antennas results in a performance loss compared to SISO. This is explained by the fact that, with cross-polarized antennas, half of the transmission power assigned to the orthogonal polarization is lost when there is only one antenna at the receiver side. As a result, the utilization of cross-polar MISO in mobile broadcasting systems has very limited applicability.

\subsubsection{Combined Time, Frequency, and Space Diversity}

While the previous section investigated the gains of time, frequency, and space diversity in a separate manner, in this section we focus on the mutual influence of each type of diversity, and also on the fading margin gains that result from the combination of different types of diversity. 


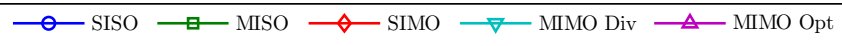
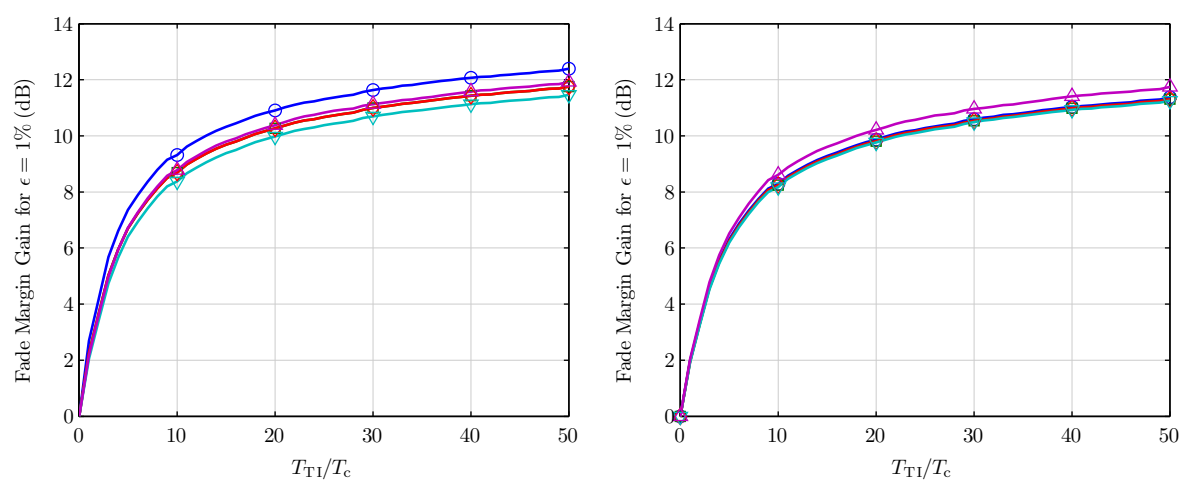

Figure 3.6: Fading margin gain of long TI with co-polar MIMO in the TU6 channel with shadowing (left), and with cross-polar MIMO in the NGH outdoor channel with shadowing (right).

Fig. 3.5 illustrates the fading margin gains that are achieved by using TI below $1 \mathrm{~s}$ with MIMO techniques. In the case of co-polarized antennas, the performance gain of TI depends on MIMO and vice versa. With interleaving durations lower than $1 \mathrm{~s}$, both TI and co-polar MIMO exploit the diversity caused by multipath propagation, so that the utilization of one technique diminishes the gain that can be achieved with the other. In contrast, the gain of MIMO with cross-polarized antennas is mostly due to better robustness against rotation and asymmetry effects, whereas the gain of TI comes from better protection against fast fading. As a result, the performance gains of TI and cross-polar MIMO do not depend on each other, and the total gain of combining both techniques is approximately equal to the sum of the individual gains. In the figure, we can also see that the advantage of MIMO optimal over MIMO diversity ( $1 \mathrm{~dB}$ at $2 \mathrm{bps} / \mathrm{Hz}$ ) is the same with any interleaving duration, which indicates that the multiplexing gain of MIMO systems is not affected by time diversity.

The influence of MIMO in the performance of long TI can be seen in Fig. 3.6. According to the results, the utilization of MIMO with cross-polarized antennas lowers the fading margin gains of TI in less than $1 \mathrm{~dB}$ for long interleaving durations. The limited influence of MIMO is explained by the fact that MIMO cannot compensate for shadowing, since the spatial correlation in this case is far beyond the separation between antennas. With cross-polarized antennas, MIMO configurations based on diversity (i.e. SIMO and diversity MIMO) do 


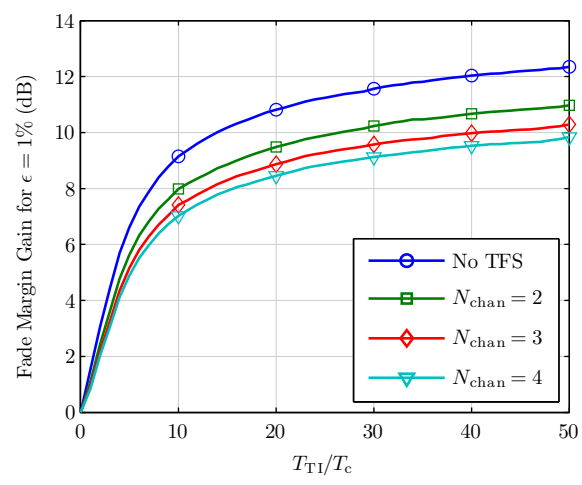

Figure 3.7: Fading margin gain of long TI with TFS $\left(f_{s}=100 \mathrm{MHz}\right)$ in the TU6 channel with shadowing.

not affect the fading margin gains of long TI as they provide very limited protection against fading. On the contrary, the gain of long TI increases in about $0.5 \mathrm{~dB}$ when it is used in combination with optimal MIMO. The reason is that the multiplexing gain of optimal MIMO depends on the average CNR encountered along the interleaving duration, and thus, it benefits from longer TI.

Compared to MIMO, the influence of TFS in the performance of long TI is significantly higher. TFS can compensate for shadowing along the user trajectory by means of better diversity in the frequency domain, and therefore, its utilization lowers the benefit of using long TI. In Fig. 3.7, we can see that the fading margin gain of long TI diminishes with the number of RF channels that are grouped in the same TFS multiplex. This is in accordance with the diminishing marginal returns of diversity: the higher the performance gain achieved with TFS, the lower the gain that is achieved with long TI.

\subsection{Fading Margin Gains of Rotated Constella- tions in BICM Systems}

\subsubsection{Methodology}

Mobile broadcasting systems employ BICM [64] to achieve error-free communication in fading channels. BICM systems using OFDM rely on the combination of FEC and interleaving to achieve diversity gains in the time and frequency domains. Furthermore, rotated constellations [25] can be used to increase the 
diversity order of BICM systems by means of SSD at the expense of increased demodulation complexity without the need of additional transmission power or bandwidth (see Section 2.3). In BICM systems, a sequence of information bits is encoded using a FEC code and is then passed through a bitwise interleaver. After this, each block of $m$ coded interleaved bits $c_{l}, l=1, \ldots, m$ is mapped to a complex data symbol $u$ in a conventional QAM constellation $\mathcal{A}$. In order to achieve SSD, a rotation is applied to the constellation symbols so that each block of $m$ bits is spread across the $D$ components of the rotated symbol $\mathbf{x}=\left[x_{1}, x_{2}, \ldots, x_{D}\right]$ in the $D$-dimensional rotated constellation $\Psi$. Then, a component interleaving is used to separate the components of the rotated symbol in the time and frequency domains so that each component faces an independent fading realization.

The outage formulation used in this chapter to obtain the fading margin gains of time, frequency and space diversity represents the best performance that can be achieved by any communication system, and does not take into consideration particular aspects of BICM systems such as the bit labelling, the constellation or the code rate. In order to evaluate the performance of rotated constellations from an information-theoretic point of view, we have computed the BICM capacity in fading channels, which corresponds to the system performance using the optimum soft MAP demodulator in (2.6) [101].

Under the assumption of full CSI at the receiver side and ideal infinite interleaving (i.e. an interleaver with length much longer than the coherence time of the channel), the D-dimensional received signal $\mathbf{y}$ in a flat fast fading channel is given by:

$$
\mathbf{y}=\mathbf{H x}+\mathbf{w},
$$

where $\mathbf{x}=\left[x_{1}, x_{2}, \ldots, x_{D}\right]^{\top}$ is the transmitted symbol, $\mathbf{H}=\operatorname{diag}\left(h_{1}, h_{2}, \ldots, h_{D}\right)$ is the channel matrix with independent fading coefficients in its diagonal, and $\mathbf{w}=\left[w_{1}, w_{2}, \ldots, w_{D}\right]^{\top}$ is the noise vector with i.i.d. circularly symmetric complex Gaussian elements of zero mean and variance $\sigma_{w}^{2}$.

In this case, the capacity obtained by using BICM, $C_{\mathrm{BICM}}$, can be computed as the mutual information $I$ between the set of transmitted code bits $c_{l}, l=$ $1, \ldots, m$ and the received signal $\mathbf{y}$, conditioned by the channel matrix $\mathbf{H}$. With i.i.d. uniform code bits, this is given by [64]:

$$
C_{\mathrm{BICM}}=\sum_{l=1}^{m} I\left(c_{l} ; \mathbf{y} \mid \mathbf{H}\right)=m-\sum_{l=1}^{m} \mathrm{E}_{\mathbf{x}, \mathbf{y}, \mathbf{H}}\left\{\log _{2} \frac{\sum_{\mathbf{x}^{\prime} \in \Psi} f\left(\mathbf{y} \mid \mathbf{x}^{\prime}, \mathbf{H}\right)}{\sum_{\mathbf{x}^{\prime} \in \chi_{l}^{b}} f\left(\mathbf{y} \mid \mathbf{x}^{\prime}, \mathbf{H}\right)}\right\},
$$




\section{CHAPTER 3. INFORMATION-THEORETIC INVESTIGATION OF DIVERSITY TECHNIQUES}

where $b \in\{0,1\}$ is equiprobable and $\chi_{l}^{b}$ denotes the set of rotated symbols in the D-dimensional rotated constellation $\Psi$ for which the code bit $c_{l}$ equals $b$. In the case of the soft-output MAP demodulator, the conditional probability density function (pdf) $f(\mathbf{y} \mid \mathbf{x}, \mathbf{H})$ corresponds to [25]:

$$
f(\mathbf{y} \mid \mathbf{x}, \mathbf{H})=\frac{1}{\pi \sigma_{w}^{2}} \exp \left(-\frac{\|\mathbf{y}-\mathbf{H} \mathbf{x}\|^{2}}{\sigma_{w}^{2}}\right),
$$

It should be noted that, for any given constellation with spectral efficiency $m$, the BICM capacity determines the FEC code rate $R_{c}$ that is needed in order to achieve error-free communication for any value of $\mathrm{CNR}^{1}$. In particular, the code rate can be derived from the BICM capacity as $R_{c}=C_{\mathrm{BICM}} / m$. Using the expression in (3.14), we have computed the code rates with the 2DRC and 4DRC included in the DVB-NGH standard, as well as with the non-rotated constellations, for a wide range of CNR values in the i.i.d. Rayleigh channel. This means that each component $h_{i}$ of the channel matrix $\mathbf{H}$ is modelled with uniform phase and Rayleigh amplitude. In addition to Rayleigh fading, we have introduced the presence of erasures as described in [102]. Despite the fact that erasure ratios lower than $15 \%$ were considered during the standardization of DVB-T2 to model the reception in SFN, a higher percentage of erasures can be realistic in the context of long TI and TFS (see Chapter 5).

\subsubsection{Information-Theoretic Results}

In left side of Fig. 3.8, we represent the gain of 2DRC and 4DRC in terms of CNR compared to non-rotated constellations for code rates ranging between 0.2 and 0.9 in the i.i.d Rayleigh channel without erasures. In the case of $4 \mathrm{DRC}$, we consider QPSK since this is the only configuration supported in the DVB-NGH standard. The results show that, broadly speaking, the additional diversity introduced by rotated constellations into the modulation scheme improves the performance of BICM for higher code rates, whereas for lower code rates, it is preferable to rely on the error-correction capabilities of the FEC code. In this manner, rotated constellations do not provide a positive gain if the code rate is low enough, and in fact, they lower the system performance in around 0.1-0.2 dB. The gain increases with higher code rates depending on the constellation, and is higher than $0 \mathrm{~dB}$ for code rates starting at $0.5(1 / 2), 0.67$ $(2 / 3)$, and $0.75(3 / 4)$ with QPSK, 16QAM and 64QAM respectively. We can also see that the gain is largest with QPSK and decreases with higher order constellations. On the other hand, 4DRC outperform 2DRC for code rates

\footnotetext{
${ }^{1}$ Under the assumption of normalized transmission symbols $\mathrm{E}\left[x x^{\top}\right]=1$, the CNR is equivalent to the inverse of the noise variance $\mathrm{CNR}=1 / \sigma_{w}^{2}$
} 

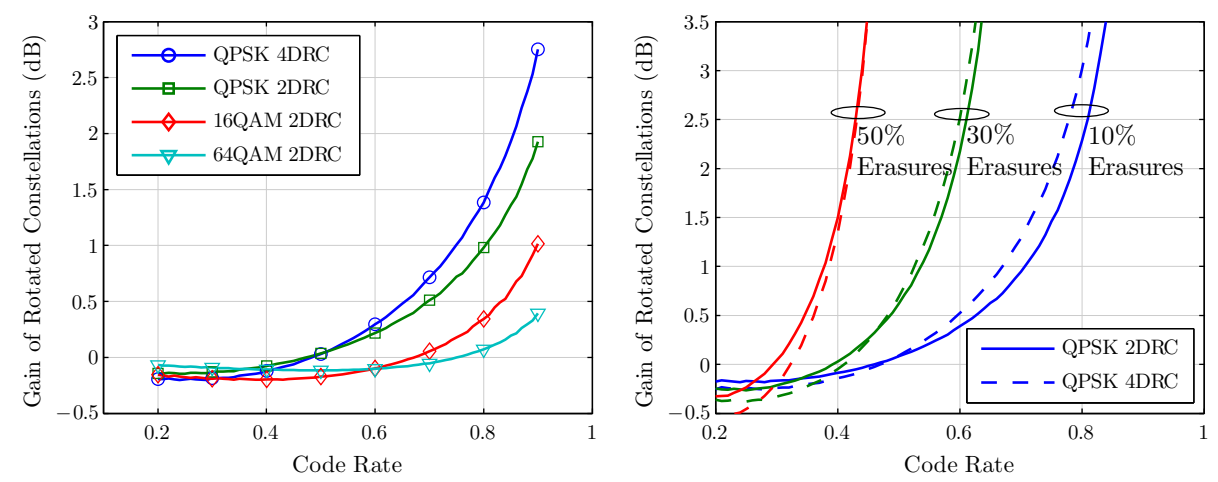

Figure 3.8: Gain of rotated constellations according to the BICM capacity in the i.i.d. Rayleigh channel without erasures (left), and with $10 \%, 30 \%$ and $50 \%$ of erasures (right).

higher than 0.5. The improvement of $4 \mathrm{DRC}$ compared to $2 \mathrm{DRC}$ increases with the code rate and can be as large as $0.8 \mathrm{~dB}$.

In the right side of Fig. 3.8, we compare the gain of $2 \mathrm{DRC}$ and $4 \mathrm{DRC}$ with QPSK in the i.i.d Rayleigh channel when a 10\%, 30\% and $50 \%$ of the information symbols are erased. According to the results, the gain of rotated constellations increases with the percentage of erasures. Also, the higher the percentage of erasures, the lower the code rate for which rotated constellations achieve a positive gain. It is important to point out that the presence of erasures limits the maximum code rate for which error-free communication is possible. With non-rotated constellations, a 10\%, 30\% and $50 \%$ of erasures require code rates lower than $0.9,0.7$ and 0.5 respectively in order to repair the loss of information. The additional diversity introduced by rotated constellations diminishes the information loss caused by erasure events, and allows error-free communication with higher code rates. Consequently, the gain of rotated constellations tends to infinity when the code rate approaches the highest value required with non-rotated constellations.

Fig. 3.9 depicts the highest code rate that is supported in BICM for errorfree communication in the i.i.d Rayleigh channel depending on the percentage of erasures. Without rotated constellations, we can see that the code rate is given by $1-P_{e}$, where $P_{e}$ is the ratio of erasures. With rotated constellations, this is no longer the case, as they support significantly higher code rates for the same percentage of erasures. It should be pointed out that the results in Fig. 3.9 can also be interpreted as the maximum percentage of erasures that are tolerated for any code rate. For example, a configuration using 2DRC or 


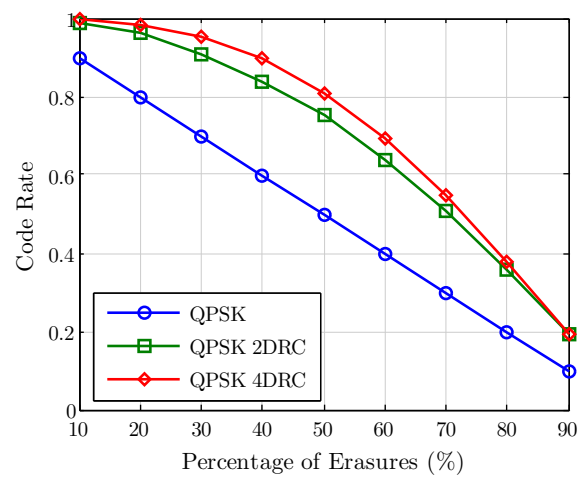

Figure 3.9: Maximum code rate supported in the i.i.d. Rayleigh channel according to the percentage of erasures.

$4 \mathrm{DRC}$ with code rate $0.33(1 / 3)$, can repair a percentage of erasures beyond $80 \%$, provided that the CNR is high enough.

\subsection{Conclusions}

In this chapter we have evaluated the use of diversity in the time, frequency and space domains from an information-theoretic point of view. To achieve this, we have approximated the performance of mobile broadcasting systems by using the outage capacity of the channel. This has allowed us to compute the fading margin gains of diversity techniques in different reception scenarios. The results based on the outage channel capacity assume the utilization of ideal FEC and interleaving, and thus, they can be considered as upper bounds of the gains that can be achieved in real systems.

First, we have obtained the fading margins gains of time, frequency, and space diversity in a separate manner. In the time domain, interleaving durations in the order of hundreds of milliseconds are sufficient to compensate for fast fading, and can achieve gains of around $5 \mathrm{~dB}$. Interleaving durations of several seconds can provide larger gains against shadowing in satellite and terrestrial scenarios. In particular, the gain of TI can be as high as $18 \mathrm{~dB}$ in satellite channels and as high as $12 \mathrm{~dB}$ in terrestrial channels. Depending on the user velocity, this might require interleaving durations too long to be practical in mobile broadcasting systems. For realistic interleaving durations, such as $10 \mathrm{~s}$, the gain of $\mathrm{TI}$ is around $8 \mathrm{~dB}$ in terrestrial scenarios, and around $12-15 \mathrm{~dB}$ in satellite scenarios. 
In the space domain, we have investigated the utilization of co-polarized antennas, which are characteristic of vehicular reception at UHF frequencies, and cross-polarized antennas, which are better suited for handheld reception. Although the gain of MIMO systems is higher in the case of co-polarized antennas, the gain with cross-polarized antennas is still significant because of improved protection against rotation and asymmetry effects. Compared to SIMO and MIMO, the gain of MISO is much more limited due to the lack of CSI in transmitters. On the other hand, the potential gain of MIMO techniques with multiplexing capabilities over MIMO techniques based only on diversity depends on the spectral efficiency. Although the improvement is negligible at $1 \mathrm{bps} / \mathrm{Hz}$, it grows linearly with the rate of information up to $5 \mathrm{~dB}$ at $6 \mathrm{bps} / \mathrm{Hz}$ and more.

In the frequency domain, the investigation has centered on TFS, and particularly, on the gains that result from better diversity against the signal variations encountered between different RF channels in the UHF band. This is achieved by grouping several RF channels in the same multiplex so that the frequency interleaving extends across hundreds of $\mathrm{MHz}$. The results reveal that the fading margin gain of TFS depends mostly on the extent of interleaving in the frequency domain, and that the number of RF channels grouped in the same multiplex does not affect the performance in a significant manner. For a realistic TFS configuration with four RF channels covering $300 \mathrm{Mhz}$ in the $\mathrm{UHF}$ band, the gain is around $6 \mathrm{~dB}$ for the highest RF channel.

In this chapter, we have also investigated the combined utilization of time, frequency and space diversity: the mutual influence of each type of diversity, and the potential gains that result from combining different types of diversity. The combination of TI with MIMO depends on whether co-polarized or crosspolarized antennas are used. With co-polarized antennas, the utilization of TI reduces the performance gain of MIMO and vice versa, as both techniques provide better protection against multipath fading. On the contrary, most of the MIMO gain with cross-polarized antennas is due to better protection against rotation and asymmetry, and thus, the combined gain of TI and cross-polar MIMO is approximately equal to the sum of the individual gains. The results also show that the multiplexing gain of MIMO systems does not depend on time diversity, and that it remains constant with the interleaving duration. Regarding the combination of TI with TFS, our investigations reveal that depending on the interleaving duration, the utilization of TFS might lower the gain of $\mathrm{TI}$ in several dBs, as both techniques can compensate for the presence of shadowing in the received signal.

Finally, we have investigated the performance of rotated constellations by using the BICM capacity in the i.i.d. Rayleigh channel with and without erasures. In particular, we have focused on the $2 \mathrm{DRC}$ and $4 \mathrm{DRC}$ adopted in the 
DVB-T2 and DVB-NGH standards. The results show that the gain of rotated constellations in BICM systems depends on the constellation and the code rate. Broadly speaking, the additional diversity introduced by rotated constellations improves the performance of BICM systems for higher code rates, whereas for low code rates, it is preferable to rely on the error-correction capabilities of the FEC code. The information-theoretic results reveal that the gain of rotated constellations is significantly larger in the presence of erasures, and that for the same code rate, they tolerate a higher percentage of erasures than non-rotated constellations. Out of the two techniques considered in this chapter, 4DRC outperform 2DRC for code rates higher than 1/2 and QPSK. 


\section{Chapter 4}

\section{Combined Diversity in Current Generation of Terrestrial Broadcasting Systems}

In this chapter, we analyze the utilization of diversity for the provision of mobile services in the first generation of terrestrial broadcasting systems standardized by the DVB, DVB-T and DVB-H, and in the second generation system DVB-T2. Due to the fact that these systems generally achieve sufficient frequency diversity in the $8 \mathrm{MHz}$ channels of the UHF band, we focus on the combined use of diversity in the time and space domains. In this context, it is important that the introduction of diversity techniques can be accomplished in a backwards compatible way, i.e. in such a way that it does not alter the operation of legacy receivers. From the point of view of network operators, it is also desirable that the introduction of diversity techniques does not require major modifications in the existent network infrastructure. For example, the need of a second transmit antenna per transmitter has been proved too costly for network operators, and is the main reason why MIMO techniques have not been implemented in terrestrial broadcasting networks till this day.

DVB-T presents two important limitations for the provision of mobile services. On one hand, it does not feature time slicing, and receivers cannot accomplish power saving by switching off their front-ends during some portions of time. On the other hand, the interleaving duration is limited to few milliseconds due to the lack of TI at the physical layer. DVB-H reuses the physical 
layer of DVB-T and incorporates additional features at the link layer in order to adapt the transmission to handheld receivers, i.e. time slicing for power saving, and MPE-FEC for time diversity. A very important disadvantage of MPE-FEC is that TI is limited to the duration of one burst, typically 100-200 ms. In this case, the utilization of multi-burst protection (e.g. MPE-iFEC) can improve the reception robustness against shadowing and extend the coverage of DVB-H networks.

Despite the fact that the physical layer of DVB-H is compatible with DVB-T, DVB-H requires the allocation of specific bandwidth for the transmission of the mobile TV content and dedicated networks to achieve good coverage levels. For this reason, we investigate the use of AL-FEC protection in DVB-T to achieve time diversity and improve the reception robustness in mobile scenarios. The main advantage of AL-FEC is that it can provide TI up to several seconds in a backwards compatible manner, i.e. without altering the operation of legacy receivers. For space diversity, we consider the implementation of multiple antennas in receivers (i.e. SIMO), as this does not require any modification in standards or transmitters. While the utilization of SIMO for handheld reception at UHF frequencies is generally impractical since the required separation between antennas is far beyond the dimensions of typical handsets, this is not an issue in the case of vehicular reception. Standard compatible MISO techniques such as DD cannot be implemented with the existent network infrastructure, and therefore, are out of the scope of this chapter.

In contrast with DVB-T, DVB-T2 features a very flexible time interleaver at the physical layer, which can provide interleaving durations ranging from few milliseconds up to several seconds. The time interleaver also supports different configurations of time diversity, power saving and zapping time by means of sub-slicing, frame skipping and inter-frame interleaving. For the first time in terrestrial broadcasting systems, DVB-T2 includes a distributed MISO technique that improves the reception in SFN, and at the same time, reuses the existent network infrastructure. DVB-T2 also supports the in-band transmission of mobile services by means of multiple PLPs or by using the T2-Lite profile that was recently included in the specification.

The rest of the chapter is structured as follows. First, in Section 4.1, we study the provision of mobile services in DVB-T and DVB-H. Here, we consider the utilization of UL-FEC for time diversity and SIMO for space diversity. In Section 4.2, we focus on DVB-T2, paying special attention to the time interleaver and the distributed MISO technique included in the standard. In Section 4.3, we compare the performance of terrestrial broadcasting DVB systems. Finally, we give some concluding remarks in Section 4.4. 


\subsection{Combined Space and Time Diversity in DVB-T}

\subsubsection{AL-FEC for Improved Mobile Reception in DVB-T}

The main idea of AL-FEC in DVB-T is to incorporate time diversity by making use of erasure codes in a backwards compatible way. In order to achieve this, the video and audio information carried in the transport streams must not be altered in any way. Moreover, the FEC parity information must be introduced in such a manner that ensures that legacy DVB-T receivers drop it without altering their proper operation.

In Fig. 4.1 we compare the operation of MPE-FEC and AL-FEC. Because of the time slicing performed in DVB-H, the interleaving duration $\left(T_{\mathrm{int}}\right)$ achieved by MPE-FEC is limited to the burst duration of around 100-200 ms. In DVB-H, when a new channel is selected by the user, the receiver must wait until the reception of the first burst with information. Assuming no other delays, the zapping time is equal to half the cycle time $\left(T_{c}\right)$ on average, and as high as one cycle time in the worst case scenario. On the other hand, DVB-T does not perform time slicing, and the services are transmitted continuously over time. The interleaving duration in AL-FEC can be configured up to several seconds, and is only restricted by memory and zapping time constraints. The zapping time in this case is equal to 1.5 times the interleaving duration on average, and as high as 2 times the interleaving duration in the worst case. In order to illustrate the advantage of AL-FEC over MPE-FEC in terms of interleaving duration and zapping time, we can consider a typical configuration of DVB-H that employs a burst duration of $200 \mathrm{~ms}$ and a cycle time of $2 \mathrm{~s}$, which results in an interleaving duration of $200 \mathrm{~ms}$ with MPE-FEC, and a zapping time of $1 \mathrm{~s}$ on average. In the case of DVB-T, a configuration with AL-FEC that provides an interleaving duration of $200 \mathrm{~ms}$ results in a zapping time of $300 \mathrm{~ms}$ on average. It should be pointed out that although DVB-T does not perform time slicing and thus, it is not possible to achieve power saving, the development of more durable batteries has reduced the power consumption issues in handheld terminals. Furthermore, for a significant portion of mobile TV receivers such as netbooks or in-car receivers, battery lifetime is of less relevance.

Raptor codes [69] are especially well suited for AL-FEC protection. Compared to the RS algorithm employed in MPE-FEC, Raptor codes are lightweight in terms of decoding complexity. This permits the FEC decoding to be done in generic software processors even on low-complexity devices without any upgrades in hardware. Another limitation of MPE-FEC that can be overcome by AL-FEC is the dependency between code rate and interleaving duration. The interleaving duration in MPE-FEC is limited to the duration of one time 


\section{CHAPTER 4. COMBINED DIVERSITY IN CURRENT SYSTEMS}

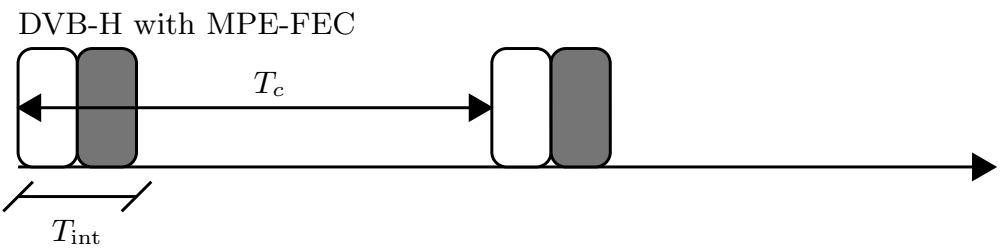

DVB-T with AL-FEC

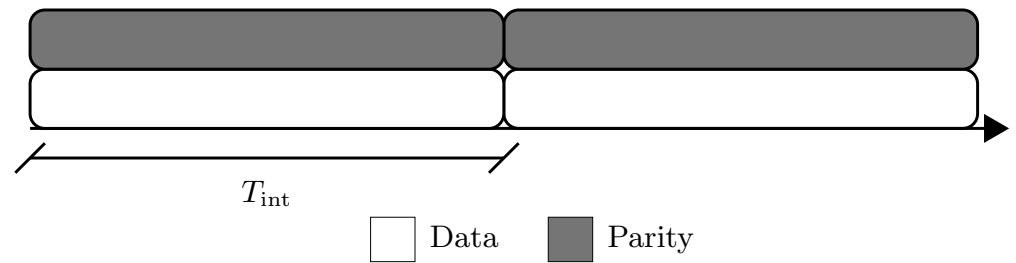

Figure 4.1: MPE-FEC in DVB-H vs. AL-FEC in DVB-T.

slicing burst, which is given by the number of rows and columns that constitute the MPE-FEC frame. While the number of rows can be freely chosen from a fixed set of values $(256,512,768$ and 1024), the maximum number of columns depends on the code rate of MPE-FEC. Due to the nature of RS encoding, in order to achieve different code rates other than the mother code rate $(3 / 4)$, it is necessary to perform a padding/puncturing mechanism that reduces the number of columns and shortens the burst duration. This is especially critical for code rates $2 / 3$ and $1 / 2$, for which the duration of each burst is respectively a $25 \%$ and a $50 \%$ shorter than in the case of code rate $3 / 4$. In contrast, the flexibility of Raptor allows AL-FEC to deliver virtually any code rate for any given interleaving duration.

\subsubsection{Simulation Results}

In the simulations, we have used the TU6 channel [95] to evaluate the gains of diversity techniques in DVB-T and DVB-H systems. This channel is representative of fast fading scenarios in the UHF band for Doppler frequencies $\left(f_{d}\right)$ higher than $10 \mathrm{~Hz}$. In the case of TI over several seconds, we have included the presence of shadowing in the received signal by assuming a user moving at constant velocity across a log-normal CNR map. The CNR map is defined by its standard deviation $(\sigma)$ and its correlation distance $\left(d_{\text {corr }}\right)$ as described in [19]. On the other hand, the results of SIMO correspond to the case of vehicular reception with two co-polarized antennas and sufficient separation so that there is no correlation between the antennas. Therefore, each antenna path between 
4.1 Combined Space and Time Diversity in DVB-T

Table 4.1: Simulation Parameters for DVB-T

\begin{tabular}{c||c}
\hline System Parameters & Value \\
\hline \hline Bandwidth & $8 \mathrm{MHz}$ \\
\hline FFT mode & $8 \mathrm{~K}$ \\
\hline Guard interval & $1 / 4$ \\
\hline MODCOD & 16QAM $1 / 2$ \\
\hline Channel estimation & Ideal \\
\hline QAM demapping & Optimum soft maximum a posteriori $(\mathrm{MAP})$ \\
\hline Cycle time in DVB-H & TS PER $1 \%$ \\
\hline QoS criterion & IP PER $1 \%$ \\
\hline Channel model & TU6 \\
& TU6 with shadowing \\
\hline Doppler $\left(f_{d}\right)$ & $10 \mathrm{~Hz}$ and $80 \mathrm{~Hz}$ \\
& Standard deviation $(\sigma) 5.5 \mathrm{~dB}$ \\
\hline Shadowing parameters & Correlation distance $\left(d_{\text {corr }}\right) 20 \mathrm{~m}$ \\
\hline \hline
\end{tabular}

the transmitter and the receiver has been modelled as an independent TU6 profile.

The parameters used for the simulations are summarized in Table 4.1. Robust configurations of MODCOD like the one shown in the table are characteristic of mobile reception, as they can provide better protection in mobile channels with adequate capacity for low data rate services. The results presented in this section have been obtained under the assumption of ideal channel estimation. In real receivers, however, some loss can be expected due to the effect of ICI, imperfect interpolation, and the presence of noise in the pilot information. The selected QoS criterion for DVB-T and DVB-H are the Transport Stream Packet Error Ratio (TSPER) 1\% and the Internet Protocol Packet Error Ratio (IPPER) 1\% respectively. Despite the fact that QoS criterions based on BER were used to evaluate the system performance in the standardization process of DVB-T [103], they only indicate the percentage of erroneous bits, and are not a proper indicator of the QoS seen by upper layers. 

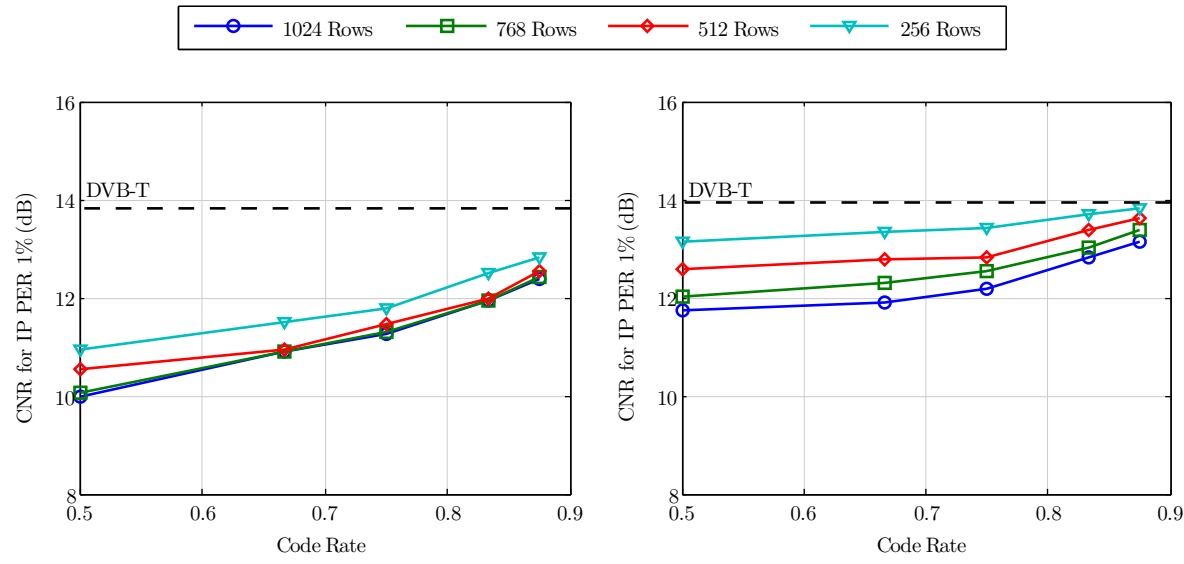

Figure 4.2: Performance of MPE-FEC in DVB-H. TU6 channel with $f_{d}=80 \mathrm{~Hz}$ (left) and $f_{d}=10 \mathrm{~Hz}$ (right). Dashed lines correspond to DVB-T without AL-FEC.

In Fig. 4.2, we represent the performance of MPE-FEC. The results have been obtained for the five code rates (i.e. $7 / 8,5 / 6,3 / 4,2 / 3$ and $1 / 2$ ) and the four values of rows (i.e. 256, 512, 768 and 1024) supported in MPE-FEC. Since the cycle time has been fixed to $1 \mathrm{~s}$ in the simulations, each configuration results in a different service data rate. In the figure, we can see that the performance of MPE-FEC depends not only on the code rate, but also on the number of rows, as this determines the duration of the TI (e.g. $200 \mathrm{~ms}$ with code rate $3 / 4$ and 1024 rows). According to the results, the maximum gain of MPE-FEC is about $4 \mathrm{~dB}$ with $80 \mathrm{~Hz}$ of Doppler and about $2 \mathrm{~dB}$ with $10 \mathrm{~Hz}$. Higher values of Doppler reduce the correlation of the channel and increase the gain of TI for the same interleaving duration as seen in Section 3.2.4.

In Fig. 4.3, we compare the performance of MPE-FEC in DVB-H and AL-FEC in DVB-T. In the case of MPE-FEC, we have selected the maximum number of rows that is allowed in the standard (1024), whereas for AL-FEC, we have selected $100 \mathrm{~ms}, 200 \mathrm{~ms}$, and $1 \mathrm{~s}$ of TI. As can be seen in the figure, the performance of MPE-FEC for code rate 3/4 is similar to that of AL-FEC with $200 \mathrm{~ms}$ of interleaving, whereas for code rates $1 / 2$ and $7 / 8$, the performance is closer to that of AL-FEC with $100 \mathrm{~ms}$ of interleaving. The results also show that while $200 \mathrm{~ms}$ of TI are sufficient to compensate for fast fading with $80 \mathrm{~Hz}$ of Doppler, $1 \mathrm{~s}$ is needed with $10 \mathrm{~Hz}$ due to the higher correlation of the channel. Consequently, the advantage of AL-FEC over MPE-FEC in terms of reception robustness grows larger with code rates other than $1 / 2$, and when the user is moving at lower velocities. 

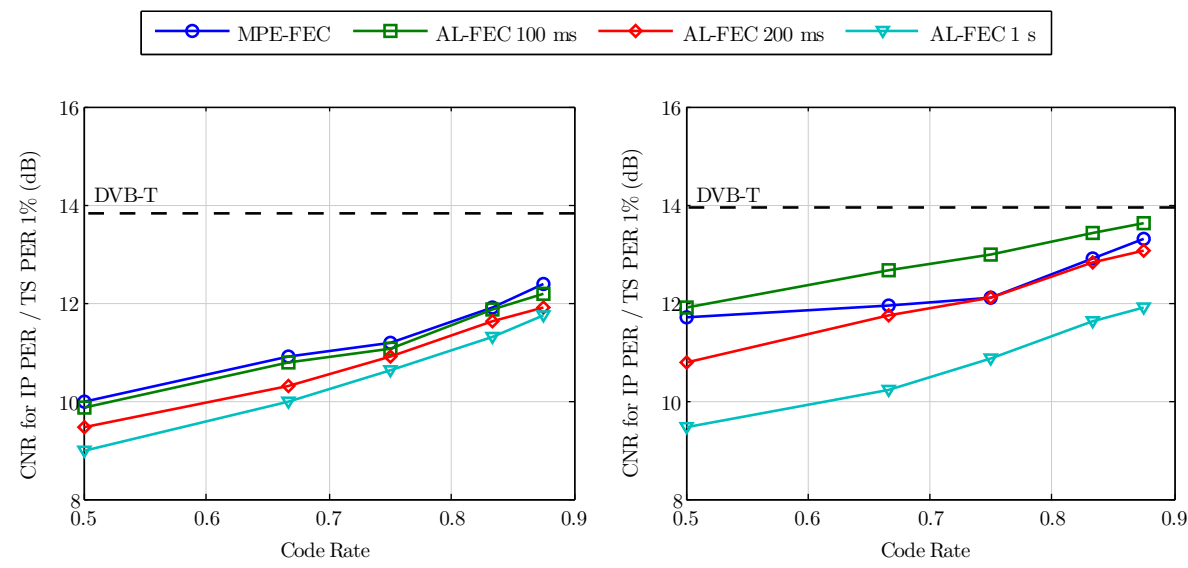

Figure 4.3: Performance comparison between MPE-FEC in DVB-H and AL-FEC in DVB-T. TU6 channel with $f_{d}=80 \mathrm{~Hz}$ (left) and $f_{d}=10 \mathrm{~Hz}$ (right). Dashed lines correspond to DVB-T without AL-FEC.

In addition to MPE-FEC, we have also investigated the utilization of interburst protection in DVB-H by means of MPE-iFEC. The two mappings currently defined for MPE-iFEC, RS and Raptor, have been configured according to the recommendations in [43]. Fig 4.4 illustrates the performance of both mappings according to the number of bursts that are interleaved. Zero bursts correspond to the case with no protection, one burst corresponds to the case with intra-burst protection similar to MPE-FEC, and more than one burst corresponds to the case with inter-burst protection. Due to the fact that the cycle time in the simulations has been configured to $1 \mathrm{~s}$, the interleaving duration ranges from $0 \mathrm{~s}$ up to a little less than $10 \mathrm{~s}$. In the figure, we can see that MPE-iFEC achieves larger gains than MPE-FEC by extending the TI across several bursts. In particular, the gain of MPE-iFEC using the lowest possible code rate $(1 / 2)$ is about $10 \mathrm{~dB}$ with $80 \mathrm{~Hz}$ of Doppler, and about $6 \mathrm{~dB}$ with $10 \mathrm{~Hz}$. Similarly to the case of MPE-FEC, the gain of MPE-iFEC increases with higher values of Doppler due to the lower channel correlation experienced by the user.

For the same code rate and interleaving, the mapping based on Raptor codes consistently outperforms the mapping based on RS. The performance gap between both mappings is approximately $1 \mathrm{~dB}$ with code rate $1 / 2$, and diminishes with higher code rates. For example, with code rate $3 / 4$, the gain of Raptor over RS is only around 0.1-0.2 dB. Raptor codes allow the encoding of very large source blocks regardless of the code rate, whereas the RS mapping is 


\section{CHAPTER 4. COMBINED DIVERSITY IN CURRENT SYSTEMS}
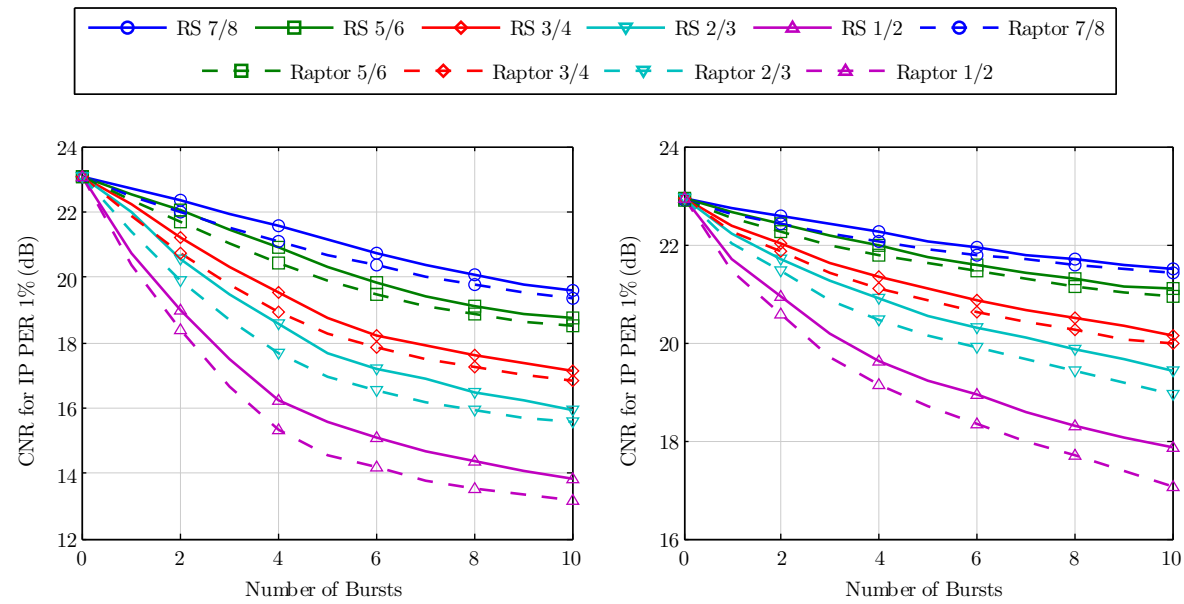

Figure 4.4: Performance of MPE-iFEC in DVB-H according to the number of time-interleaved bursts in the TU6 channel with shadowing $\left(f_{d}=80 \mathrm{~Hz}\right.$ left, $f_{d}=10 \mathrm{~Hz}$ right, $\sigma=5.5 \mathrm{~dB}$ and $d_{\text {corr }}=20 \mathrm{~m}$ ).

based on the puncturing and padding of an encoding matrix in the same manner as MPE-FEC. As a result, the mapping based on Raptor codes provides better error correction capabilities and higher flexibility in terms of service data rate, cycle time, and code rate.

The results presented until this point have only considered the benefits of time diversity by means of UL-FEC techniques. In Fig. 4.5, we investigate the combined use of diversity in the time and space domains by incorporating AL-FEC protection and SIMO into DVB-T. In the left side of the figure, the results focus on fast fading and correspond to interleaving durations lower than $1 \mathrm{~s}$ with AL-FEC. In the right side of the figure, the results account for the presence of fast fading and shadowing in the received signal and correspond to interleaving durations up to several seconds. In both cases, the MODCOD at the physical layer has been configured to 16QAM $1 / 2$, whereas the code rate of AL-FEC is $1 / 2$. This corresponds to a spectral efficiency of 1 bit per subcarrier (cell). According to the results, the gain of AL-FEC with interleaving durations below $1 \mathrm{~s}$ can be as high as $5 \mathrm{~dB}$, whereas the gain of SIMO is about $6 \mathrm{~dB}$. The combined gain of AL-FEC and SIMO is approximately $9 \mathrm{~dB}$. In the case of fast fading, both SIMO and AL-FEC exploit the diversity caused by multi-path propagation, and the utilization of one technique diminishes the performance of the other. As a result, the gains of AL-FEC and SIMO do not add on each 


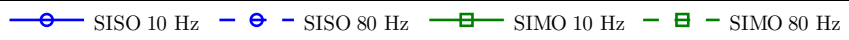
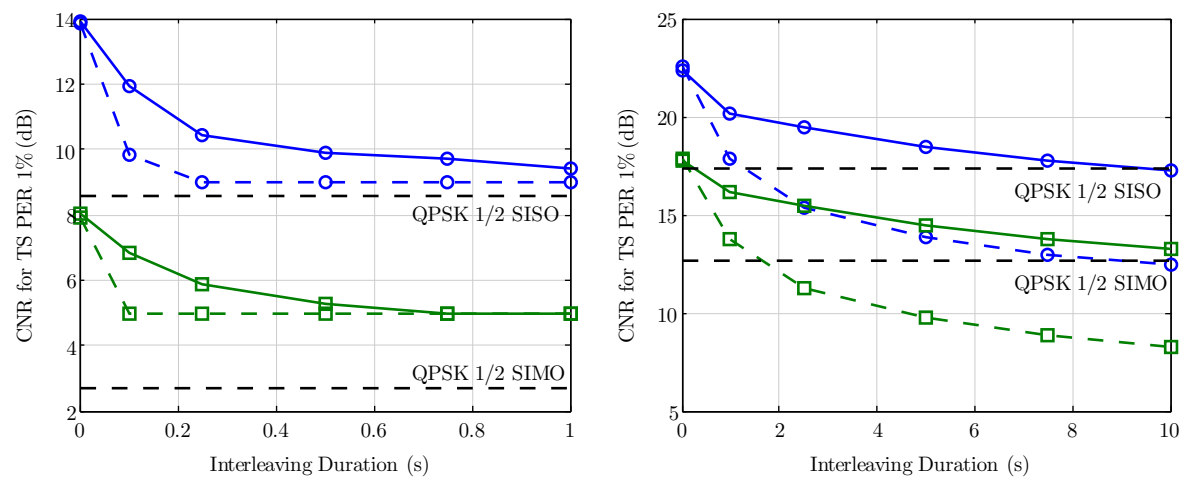

Figure 4.5: Performance of DVB-T with AL-FEC and SIMO. On the left, interleaving durations up to $1 \mathrm{~s}$ in the TU6 channel. On the right, interleaving durations up to $10 \mathrm{~s}$ in the TU6 channel with shadowing $\left(\sigma=5.5 \mathrm{~dB}\right.$ and $\left.d_{\mathrm{corr}}=20 \mathrm{~m}\right)$.

other, and the improvement of using both techniques is lower than the sum of the individual gains.

The gain of AL-FEC with interleaving durations up to $10 \mathrm{~s}$ is approximately 5-10 dB depending on the Doppler, whereas the combined gain of AL-FEC and SIMO is approximately 9-14 dB. While SIMO provides higher gains than AL-FEC with interleaving durations lower than $1 \mathrm{~s}$, the utilization of AL-FEC with interleaving durations up to several seconds is generally more effective than SIMO. The reason is that AL-FEC can provide large gains against shadowing in the time domain, whereas multiple receive antennas cannot be used to average the shadowing in the space domain.

It is important to note that UL-FEC techniques do not provide any gain in stationary reception, and that the improvement in mobile channels comes at the expense of reduced capacity due to the transmission of additional parity data. The straight dashed lines in Fig. 4.5 correspond to a configuration with QPSK $1 / 2$ at the physical layer without AL-FEC, providing the same spectral efficiency of 1 bits per cell (bpc). For interleaving durations below $1 \mathrm{~s}$ (left side of the figure), lowering the MODCOD at the physical layer instead of using AL-FEC achieves better performance. In contrast, the use of AL-FEC with interleaving durations higher than $1 \mathrm{~s}$ (right side of the figure) is preferable to a more robust MODCOD configuration at the physical layer, as it enables the reception at lower CNR values. From these results, we can conclude that the utilization of UL-FEC techniques in DVB-T and DVB-H is only interesting 


\section{CHAPTER 4. COMBINED DIVERSITY IN CURRENT SYSTEMS}

for interleaving durations up to several seconds, or when it is not possible to increase the reception robustness at the physical layer by means of lower code rates and constellations.

\subsubsection{Network Planning Discussion}

In this section, we investigate the benefits of diversity techniques in terms of network planning and whether the provision of mobile services in DVB-T networks planned for fixed and portable indoor reception is feasible. Despite the fact that diversity techniques can provide important gains against fading, mobile reception is also challenged by the use of low-gain antennas at ground level in outdoor and indoor scenarios. Table 4.2 shows the link margin that is necessary in DVB-T networks for fixed reception (rooftop antennas), portable indoor reception (external antennas in portable devices), vehicular reception (external antennas in vehicles), and handheld indoor reception (integrated antennas in handsets) in the UHF band. The values listed in the table correspond to those presented in [99]. Compared to fixed reception, which is typically performed with rooftop antennas at $10 \mathrm{~m}$, there is a height loss resulting from the low antenna height of about $1.5 \mathrm{~m}$, a loss in the antenna gain caused by the utilization of omnidirectional and integrated antennas, and a building penetration loss in indoor scenarios. On the other hand, the presence of multipath fading in the received signal causes a loss of $0.6 \mathrm{~dB}$ for fixed reception (Rice F1 channel), a loss of $2.4 \mathrm{~dB}$ for portable and handheld indoor reception (Rayleigh P1 channel), and a loss of $5 \mathrm{~dB}$ for vehicular reception (TU6 channel) compared to AWGN (see Section 4.1.2 and [1]). Although it is not listed in the table, there is an additional loss in the vehicular scenario due to the ICI caused by the Doppler spread. This degradation limits the maximum velocity of users depending on the transmission mode, particularly the Fast Fourier Transform (FFT) size and the pilot pattern, and also on the quality of the channel estimation performed at the receiver side.

Terrestrial broadcasting networks can employ low code rates and small constellations to improve the coverage of mobile services. In networks planned for fixed reception, switching from a typical configuration such as 64QAM 2/3 to the most robust configuration supported in the standard (QPSK 1/2) results in a link margin gain of around $13.2 \mathrm{~dB}$ (Rice F1 channel). In networks planned for portable indoor reception, using QPSK $1 / 2$ instead of 16QAM $2 / 3$ results in a link margin gain of around $9.4 \mathrm{~dB}$ (Rayleigh P1 channel) [1]. DVB-T networks can dedicate an entire RF channel for the transmission of mobile services, or alternatively, they can use Hierarchical Modulation (HM) to combine fixed and mobile services in the same RF channel [104]. HM multiplexes two different streams into the same constellation, each of them with a particular ro- 
4.1 Combined Space and Time Diversity in DVB-T

Table 4.2: Link Budget of DVB-T networks in the UHF band

\begin{tabular}{c||c|c|c|c}
\hline & Fixed & $\begin{array}{c}\text { Portable } \\
\text { Indoor }\end{array}$ & Vehicular & $\begin{array}{c}\text { Handheld } \\
\text { Indoor }\end{array}$ \\
\hline \hline Multi-path fading loss $^{1}$ & $0.6 \mathrm{~dB}$ & $2.4 \mathrm{~dB}$ & $5 \mathrm{~dB}$ & $2.4 \mathrm{~dB}$ \\
\hline Height loss $^{2}$ & $0 \mathrm{~dB}$ & $23.5 \mathrm{~dB}$ & $23.5 \mathrm{~dB}$ & $23.5 \mathrm{~dB}$ \\
\hline Feeder loss & $4 \mathrm{~dB}$ & $0 \mathrm{~dB}$ & $0 \mathrm{~dB}$ & $0 \mathrm{~dB}$ \\
\hline Building penetration loss $^{\prime}$ & $0 \mathrm{~dB}$ & $11 \mathrm{~dB}$ & $0 \mathrm{~dB}$ & $11 \mathrm{~dB}$ \\
\hline Antenna gain $^{3}$ & $11 \mathrm{~dB}$ & $0 \mathrm{~dB}$ & $0 \mathrm{~dB}$ & $-9.5 \mathrm{~dB}$ \\
\hline \hline Link margin total & $2.6 \mathrm{~dB}$ & $49.9 \mathrm{~dB}$ & $41.5 \mathrm{~dB}$ & $59.4 \mathrm{~dB}$ \\
\hline Distance to fixed & - & $-47.3 \mathrm{~dB}$ & $-38.9 \mathrm{~dB}$ & $-56.8 \mathrm{~dB}$ \\
\hline Distance to portable indoor & - & - & $8.4 \mathrm{~dB}$ & $-9.5 \mathrm{~dB}$ \\
\hline
\end{tabular}

bustness. For example, a 64QAM constellation can provide a robustness similar to QPSK for one stream (called High Priority (HP) stream), and a robustness similar to 16QAM for another stream (called Low Priority (LP) stream). The utilization of HM, however, losses some performance due to the multiplexing of different streams into the same constellation. This means that both the HP and LP streams are transmitted with a lower robustness compared to the equivalent constellation in non-HM mode. In this manner, HM can achieve a gain of $10.2 \mathrm{~dB}$ in the HP stream (e.g. mobile reception) by using a QPSK $1 / 2$ configuration inside of a non-uniform 64QAM constellation. This results in a performance penalty of around $2.6 \mathrm{~dB}$ for the LP stream (e.g. fixed reception) compared to the non-hierarchical 64QAM $2 / 3$ configuration.

The location correction factor that appears in Table 4.2 is required to ensure a minimum percentage of coverage at the edges of the service area. The presence of shadowing makes coverage predictions statistical, such that the coverage probability rather than the signal level is used for network planning purposes. Assuming that shadowing follows a log normal distribution with zero mean

\footnotetext{
${ }^{1}$ Compared to AWGN according to the values presented in [1]. The MODCOD is assumed to be 64QAM 2/3 in fixed reception, and QPSK 1/2 in portable indoor, vehicular, and handheld indoor reception

${ }^{2}$ Height loss corresponds to the urban scenario.

${ }^{3}$ Antenna gain relative to half wave dipole.

${ }^{4}$ Location correction factor corresponds to good coverage quality (i.e. $95 \%$ in fixed, portable indoor, and handheld indoor, and $99 \%$ in vehicular).
} 
and standard deviation $\sigma$, the location correction factor, $C_{l}$, that is required to achieve a certain coverage probability can be computed as [103]:

$$
C_{l}=\mu \times \sigma,
$$

where $\mu$ is the distribution factor. The coverage probability for any given distribution factor is given by [105]:

$$
\text { Coverage Probability }=1-\frac{1}{2} \operatorname{erfc}\left(\frac{\mu}{\sqrt{2}}\right) \text {, }
$$

where erfc( $)$ is the complementary error function. For $\sigma=5.5 \mathrm{~dB}$, location correction factors of $3 \mathrm{~dB}, 7 \mathrm{~dB}, 9 \mathrm{~dB}$, and $13 \mathrm{~dB}$ achieve a coverage probability of $70 \%, 90 \%, 95 \%$, and 99\%. Typical targets in terrestrial DVB systems are 95\% for good portable reception, and $99 \%$ for good vehicular reception.

According to Table 4.2, the distance to the link margin needed for fixed reception is $47.3 \mathrm{~dB}$ for portable indoor reception, $38.9 \mathrm{~dB}$ for vehicular reception, and $56.8 \mathrm{~dB}$ for handheld indoor reception. Such high values cannot be compensated with the combined use of SIMO, UL-FEC, and more robust MODCOD, and thus, good coverage levels of mobile services cannot be achieved in DVB-T networks planned for fixed reception. This can be see in Table 4.3, where it is shown that the coverage of vehicular reception and handheld indoor is below acceptable values ( $90 \%$ and $70 \%$ respectively). As a result, the provision of mobile services in networks planned for fixed reception can only be performed as a best effort kind of service that is available in areas with good coverage conditions.

DVB-T networks planned for portable indoor reception take into account the penalization due to the height loss, the use of low gain antennas, and the building penetration loss. As it is shown in Table 4.3, these networks provide full coverage for vehicular reception, and achieve close to good coverage levels for handheld indoor reception (95\%) without the use of UL-FEC. In the case of vehicular reception, the link margin gains of diversity techniques can be traded for better spectral efficiency by using higher code rates and/or larger constellations. This means that for the same capacity, it is possible to transmit a higher number of services targeting vehicular receivers, or alternatively, the same number of services can be fit into a smaller bandwidth. With a single receive antenna, vehicular services can be transmitted using 64QAM 3/4 and achieve good coverage (99\%); with two receive antennas, it is possible to use 64QAM 7/8 and obtain a 17\% gain in terms of spectral efficiency.

In order to illustrate the advantage of diversity techniques in terms of network planning, it is possible to translate the link margin gains computed in this chapter into a coverage extension of mobile services in terrestrial broad- 
4.1 Combined Space and Time Diversity in DVB-T

Table 4.3: Coverage probability of mobile services in DVB-T networks

\begin{tabular}{c||c|c|c|c}
\hline \multirow{2}{*}{$\begin{array}{c}\text { Diversity } \\
\text { Techniques }\end{array}$} & \multicolumn{2}{c|}{$\begin{array}{c}\text { Network planned for } \\
\text { fixed reception }\end{array}$} & \multicolumn{2}{c}{$\begin{array}{c}\text { Network planned for } \\
\text { portable indoor }^{6}\end{array}$} \\
\cline { 2 - 5 } & Vehicular & $\begin{array}{c}\text { Handheld }^{\text {Indoor }} \\
\text { Indicular }\end{array}$ & $\begin{array}{c}\text { Handheld } \\
\text { Indoor }\end{array}$ \\
\hline \hline- & $1 \%$ & $0 \%$ & $100 \%$ & $94 \%$ \\
\hline SIMO & $11 \%$ & $0 \%$ & $100 \%$ & - \\
\hline UL-FEC 1/2 250 ms & $1 \%-8 \%$ & $0 \%$ & $100 \%$ & $94 \%-96 \%$ \\
\hline UL-FEC 1/2 10 s & $1 \%-31 \%$ & $0 \%$ & $100 \%$ & $94 \%-99 \%$ \\
\hline SIMO + & $11 \%-25 \%$ & $0 \%$ & $100 \%$ & - \\
\hline UL-FEC 1/2 250 ms & & & & - \\
\hline SIMO + & $11 \%-59 \%$ & $0 \%$ & $100 \%$ & \\
\hline
\end{tabular}

casting networks. Following the methodology presented in [105], the received signal strength decreases exponentially with the distance so that the distance dependent path loss, $L_{d}$, can be expressed as:

$$
L_{d}=L_{0}+10 \alpha \log _{10}(d),
$$

where $L_{0}$ represents the path loss at $1 \mathrm{~km}$ distance, $\alpha$ is the propagation exponent, and $d$ is the distance in $\mathrm{km}$. The relative increase in the maximum distance (cell radius), $\Delta R$, for any given link margin gain in $\mathrm{dB}, \Delta L$, can be computed as:

$$
\frac{R+\Delta R}{R}=10^{\Delta L(10 \alpha)}
$$

Assuming a cell of circular shape, the gain in coverage area is equal to the square of the cell radius gain. In Table 4.4, we represent the link margin gain obtained by each diversity technique, and the corresponding coverage extension, for $\alpha=3.5$ (typical urban environment). These values can be also interpreted as a reduction of the network infrastructure that is required for the provision of mobile services. We can see that the incorporation of two receive antennas in vehicles can multiply by a factor higher than two the coverage area of mobile

\footnotetext{
${ }^{5}$ The values have been obtained assuming 64QAM 2/3 for fixed reception and QPSK $1 / 2$ for mobile and handheld indoor reception.

${ }^{6}$ The values have been obtained assuming 16QAM 2/3 for fixed reception and QPSK 1/2 for mobile and handheld indoor reception.
} 


\section{CHAPTER 4. COMBINED DIVERSITY IN CURRENT SYSTEMS}

Table 4.4: Coverage extension of mobile services in DVB-T networks ${ }^{7}$

\begin{tabular}{c||c|c}
\hline $\begin{array}{c}\text { Diversity } \\
\text { Technique }\end{array}$ & $\begin{array}{c}\text { Link Margin } \\
\text { Gain }\end{array}$ & $\begin{array}{c}\text { Coverage } \\
\text { Extension }\end{array}$ \\
\hline \hline SIMO & $6 \mathrm{~dB}$ & $120 \%$ \\
\hline UL-FEC $1 / 2250 \mathrm{~ms}$ & $0 \mathrm{~dB}-5 \mathrm{~dB}$ & $0 \%-93 \%$ \\
\hline UL-FEC $1 / 210 \mathrm{~s}$ & $0 \mathrm{~dB}-10 \mathrm{~dB}$ & $0 \%-273 \%$ \\
\hline SIMO + UL-FEC $1 / 2250 \mathrm{~ms}$ & $6 \mathrm{~dB}-9 \mathrm{~dB}$ & $120 \%-227 \%$ \\
\hline SIMO + UL-FEC $1 / 210 \mathrm{~s}$ & $6 \mathrm{~dB}-14 \mathrm{~dB}$ & $120 \%-531 \%$ \\
\hline
\end{tabular}

services in DVB-T networks. On the other hand, the performance of UL-FEC depends on the user velocity and route across the service area. The values listed on the table vary between the case when the user is not moving (no gain) and the case in which the user is traveling at a constant velocity of $144 \mathrm{~km} / \mathrm{h}$ (largest gain). In order to quantify the coverage extension of UL-FEC in a precise manner, it is necessary to perform dynamic system-level simulations that take into account the mobility of the users in realistic scenarios [106].

\subsection{Combined Space and Time Diversity in DVB-T2}

\subsubsection{Analysis of TI in DVB-T2}

While the TI adopted in DVB-T2 was described in general terms in Section 2.4, in this section we study the trade-offs that are possible in terms of time diversity, data rate, latency and power saving. We also review the lack of TI for the signalling information in DVB-T2, and the mechanisms that can be used for increasing its robustness in mobile scenarios. In addition, we introduce the utilization of UL-FEC for the provision of long TI.

\section{Trade-off between Time Diversity and Data Rate}

In DVB-T2, the maximum interleaving duration is limited by the service data rate and the amount of TI memory that is available in receivers. This has

\footnotetext{
${ }^{7}$ The gains of SIMO correspond to a implementation based on two co-polar antennas with sufficient separation between the antennas. The gains of UL-FEC depend on the user velocity and route across the service area. The highest value corresponds to the case when the user is moving at a constant velocity of $144 \mathrm{~km} / \mathrm{h}$.
} 


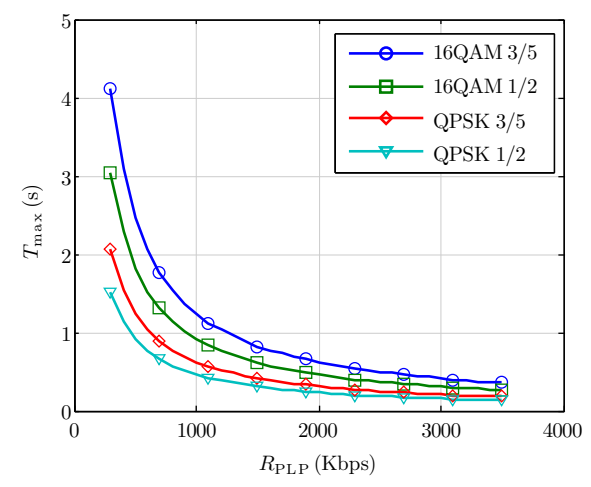

Figure 4.6: Maximum interleaving duration in DVB-T2.

been set in the standard to $2^{19}+2^{15}$ cells as a compromise between hardware complexity and performance. Assuming that a cell requires two 10-bit values (for the real and imaginary part) together with a few bits for CSI, the memory size is around $12 \mathrm{Mb}$. In DVB-T2, the number of cells that can be fit in the TI memory is the same for every configuration of MODCOD, and therefore, the maximum interleaving duration for any PLP increases with higher order constellations and lower code rates, as in this case, more data bits are carried per cell. The maximum interleaving duration in DVB-T2 can be computed as:

$$
T_{\max } \approx \frac{\left\lfloor\frac{M_{\mathrm{TI}} \times \eta_{\mathrm{MOD}}}{N_{\mathrm{LDPC}}}\right\rfloor \times\left(K_{\mathrm{BCH}}-80\right)}{R_{\mathrm{PLP}}},
$$

where $M_{\mathrm{TI}}$ is the number of cells that can be stored in the TI memory, $\eta_{\mathrm{MOD}}$ is the number of bits per constellation symbol (e.g., 2 bits for QPSK), $N_{\text {LDPC }}$ is the number of encoded bits per LDPC codeword (e.g. 16200 bits for 16K LDPC codes), $K_{\mathrm{BCH}}$ is the number of source bits per BCH codeword (e.g. 7032 bits for code rate $1 / 2$ and $16 \mathrm{~K}$ LDPC codes), and $R_{\mathrm{PLP}}$ is the data rate of any PLP carried in the $\mathrm{T} 2$ signal (in bps). The maximum interleaving duration that is supported in DVB-T2 as a function of the data rate is shown in Fig. 4.6. According to the figure, interleaving durations of several seconds with a robust configuration of MODCOD are only possible for data rates below $1 \mathrm{Mbps}$.

\section{Trade-off between Time Diversity and Latency}

In DVB-T2, receivers have to wait until the complete reception of one TI block before they can deinterleave it and process the FEC blocks. The average 


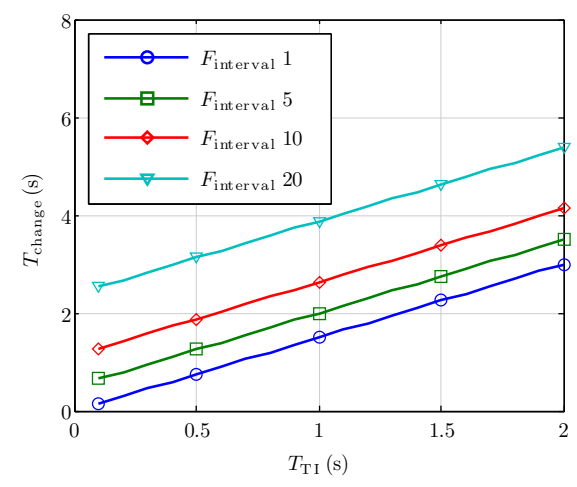

Figure 4.7: Average zapping time in DVB-T2.

zapping time that a receiver must wait until the complete reception of the first TI block is given by:

$$
T_{\text {change }}=T_{\text {wait }}+T_{\text {int }},
$$

where $T_{\text {int }}$ is the interleaving duration and $T_{\text {wait }}$ is the average period of time until the arrival of the first T2 frame carrying the beginning of a TI block. From (4.6), we can see that the longer the interleaving duration, the longer the receivers must wait before decoding the service. With a single PLP, the interleaving duration is around $70 \mathrm{~ms}$, whereas with multiple PLPs, the interleaving duration can be extended to the full T2 frame duration (up to $250 \mathrm{~ms}$ ) by means of sub-slicing, and across several frames by means of inter-frame interleaving. It should be pointed out that the maximum interleaving duration in DVB-T2 is limited by the data rate according to Eq. (4.5).

For the sake of simplicity, we assume that the PLPs are distributed across the total length of the T2 frames by means of sub-slicing. If this is not the case, the values presented here may differ in +/- one T2 frame depending on the position of the PLPs within the frame. Also, the transmission of Future Extension Frame (FEF) parts is not included. With these considerations in mind, the value of $T_{\text {wait }}$ can be approximated by:

$$
T_{\text {wait }} \approx \frac{T_{\mathrm{F}} \times F_{\text {interval }} \times N_{\text {frames }}}{2},
$$

where $T_{\mathrm{F}}$ is the length of the T2 frame, $F_{\text {interval }}$ is the frame interval and $N_{\text {frames }}$ is the number of frames that are interleaved together in the same TI block. On the other hand, $T_{\text {int }}$ can be estimated as: 


$$
T_{\text {int }} \approx T_{\mathrm{F}} \times\left(F_{\text {interval }} \times\left(N_{\text {frames }}-1\right)+1\right) .
$$

The zapping time vs. the interleaving duration is represented in Fig. 4.7 for different values of frame interval. We have considered a frame length of $250 \mathrm{~ms}$, which corresponds to the highest value allowed in the standard. According to the figure, tolerable zapping times (i.e. $<2 \mathrm{~s}$ ) are not possible when interleaving durations higher than $1 \mathrm{~s}$ are used at the physical layer. Also, the utilization of frame skipping affects the zapping time in a negative manner, as it increases the time that the receiver must wait until the arrival of the first information frame.

\section{Trade-off between Time Diversity and Power Saving}

DVB-T2 receivers can switch off some of their circuitry during the periods of time between time slices in order to reduce power consumption. From a power saving point of view, each PLP should be transmitted in a single time slice of information per T2 frame. The main reason for this is that due to synchronization and channel estimation issues, receivers need to wake up a period of time prior to the actual reception of each time slice. In contrast, the transmission of multiple sub-slices per T2 frame improves the robustness against fast fading by means of better time diversity. The higher the number of sub-slices, the better the diversity, but the lower the power saving that can be achieved at the receiver side. If the number of sub-slices is high enough, receivers need to remain powered during the reception of the entire T2 frame. When frame skipping is used, receivers can still reduce the power consumption in the periods of time between the reception of two frames with service information. It must be noted, however, that depending on the cycle time between frames, this can result in worse robustness against shadowing.

Assuming that the PLPs are distributed across the total length of the T2 frames by means of sub-slicing and that no FEF parts are transmitted, the power saving in DVB-T2 can be approximated by:

$$
P_{\text {saving }} \approx 100 \times\left(1-\frac{\min \left\{T_{\mathrm{F}}, N_{\text {subslices }} \times\left(T_{\text {synchro }}+T_{\text {slice }}\right)\right\}}{F_{\text {interval }} \times T_{\mathrm{F}}}\right)
$$

where $N_{\text {subslices }}$ is the number of sub-slices in one T2 frame, $T_{\text {synchro }}$ is the time required for synchronization, $T_{\text {slice }}$ is the duration of one sub-slice, $F_{\text {interval }}$ is the frame interval and $T_{\mathrm{F}}$ is the length of the $\mathrm{T} 2$ frame.

Fig. 4.8 illustrates the power saving for different combinations of sub-slicing and frame interval. The results are given for $8 \mathrm{~K}$ FFT, guard interval 1/4, PP1, and frame length $250 \mathrm{~ms}$. It has been assumed that that a receiver needs 


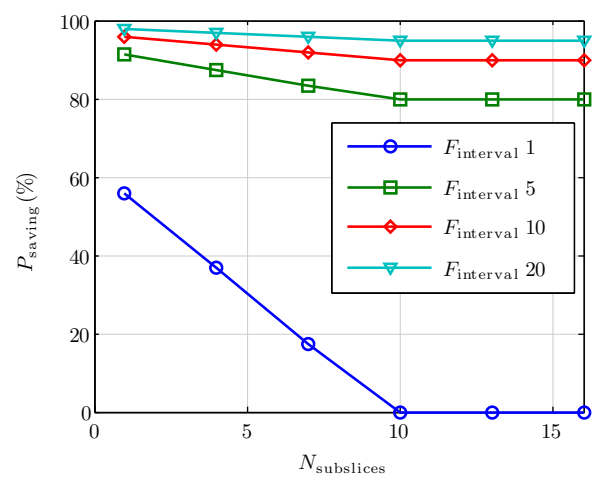

Figure 4.8: Power saving in DVB-T2.

$15 \mathrm{~ms}$ to wake up and start receiving each time slice. A total of $2^{19}$ cells of data from a PLP are transmitted in each frame. In the figure, we can see that the power saving diminishes with the number of sub-slices per frame, and that values higher than $90 \%$ cannot be achieved without the use of frame interval. It should be pointed out that a faster wake up time and a lower number of cells transmitted per PLP (e.g. due to lower data rates or the utilization of inter-frame interleaving), will result in better power saving values than those shown in Fig. 4.8.

\section{UL-FEC Protection in DVB-T2}

UL-FEC can be introduced in DVB-T2 to cope with the limitations of the physical layer for long TI in terms of TI memory and zapping time. In this case, the main idea is to combine the powerful protection at the physical layer against noise and fast fading, with the long time interleaving protection that can be provided at upper layers against shadowing. In order to optimize the system performance, the interleaving duration at the physical layer should be in the order of hundreds of milliseconds, whereas long TI up to several seconds should be provided at upper layers. In particular, an AL-FEC implementation similar to the one described in Section 4.1 could be used for the protection of TS packets in DVB-T2.

\subsubsection{Distributed MISO in DVB-T2}

The arrival of similar-strength signals from different transmitters in LoS scenarios (e.g. $0 \mathrm{~dB}$ echo) can cause deep notches in the frequency response of the 
channel. Similarly to fading, these notches can erase a significant percentage of subcarriers and degrade the QoS in an important manner. The distributed MISO technique included in DVB-T2 employs the Alamouti code between pairs of transmitters to combine the signals in an optimum way and remove the presence of notches from the channel. This technique can also improve the reception in nLoS scenarios as a result of better diversity. The main advantage of distributed MISO is that it can improve the reception robustness in SFN without the need of additional network infrastructure. It should be pointed out that in order to use distributed MISO in DVB-T2 the density of pilots needs to be doubled for the same robustness in channel estimation. This overhead should be taken into account when evaluating the improvement of distributed MISO in terms of spectral efficiency.

\subsubsection{Robustness of the Signalling Information in DVB-T2}

The physical layer signalling in DVB-T2 is transmitted inside preamble symbols known as P1 and P2 at the beginning of each T2 frame. The P1 symbol is the first OFDM symbol transmitted in the T2 frames, and is intended for fast identification of available T2 signals. At the same time, it also enables the reception of the P2 symbols in a very robust way even on mobile channels. P2 symbols are transmitted right after the P1 symbol and carry the Layer 1 (L1) signalling. The L1-pre signalling consists of very basic information (200 bits), and is always transmitted with the most robust configuration available (Binary Phase-Shift Keying (BPSK) and code rate 1/5). The L1-post signalling enables the reception of the actual data and is transmitted with modulations BPSK, QPSK, 16QAM or 64QAM and code rate $1 / 2$. By means of different constellations, it is possible to adjust the transmission of the L1-post signalling so that it is more robust than the data (about $3 \mathrm{~dB}$ ), and at the same time, results in the lowest possible overhead. The L1-post signalling is divided in the configurable and the dynamic part. The configurable part signals the number of PLPs and their configuration parameters, and is seldom changed during the transmission of $\mathrm{T} 2$ signals. The dynamic part signals the position of the PLPs within the frames and changes from frame to frame. In order to maximize the time diversity, the codewords with the L1 signalling information are uniformly distributed over all the P2 symbols of one T2 frame (e.g. 2 P2 symbols are used in the $8 \mathrm{~K}$ FFT mode). This accounts for an interleaving duration similar to that of DVB-T (i.e. in the order of milliseconds), which may not be sufficient in mobile scenarios. For example, the interleaving duration of the L1 signalling for $8 \mathrm{~K}$ OFDM symbols in $8 \mathrm{MHz}$ channels is around $2 \mathrm{~ms}$ (1 ms per OFDM symbol). 
DVB-T2 includes two mechanisms for increasing the robustness of the L1 signalling known as L1 repetition and in-band signalling. The former increases the robustness of the L1 signalling by transmitting in each T2 frame the L1 dynamic part that corresponds to the current and the next T2 frame. The latter transmits the L1 dynamic part embedded in the data path so that it possesses the same robustness as the data. Although the utilization of L1 repetition and in-band signalling introduces a delay in transmission of one T2 frame and one interleaving frame respectively, it does not result in an increase of the zapping time. In addition to L1 repetition and in-band signalling, advanced receivers can improve the robustness of the L1 signalling by reusing the information that has been decoded successfully in previous frames and that is also transmitted in the current frame when decoding the codewords. This is the case of the L1 configurable part, which generally remains the same from frame to frame. If L1 repetition is used, this technique can also be applied to the L1 dynamic part of the current frame, provided that the L1 signalling was successfully decoded in the previous frame. It must be noted that none of these techniques improves the robustness of the L1 signalling for the reception of the first frame.

\subsubsection{Simulation Results}

The parameters used for the evaluation of DVB-T2 are similar to those presented in Table 4.1. In this case, the selected QoS criterion is the Base Band Frame Error Ratio (BBFER) 1\%, which is comparable to the TSPER $1 \%$ and the IPPER $1 \%$ in the case of DVB-T and DVB-H systems. Regarding the utilization of TI, the simulations assume the transmission of $2^{18}$ cells per interleaving frame, which represents half of the TI memory in DVB-T2, and the entire TI memory in T2-Lite. Unless stated otherwise, the results have been obtained with constellation QPSK and code rate $1 / 2$.

\section{Data Path}

The simulation results presented in Fig. 4.9 show that the gain of sub-slicing is about $1 \mathrm{~dB}$ with $80 \mathrm{~Hz}$ of Doppler and about $2 \mathrm{~dB}$ with $10 \mathrm{~Hz}$. If sub-slicing is not enabled, the interleaving duration is limited by the number of cells that are transmitted per frame $(\approx 50 \mathrm{~ms})$, whereas with sub-slicing, the interleaving duration is given by the frame length $(\leq 250 \mathrm{~ms})$. From the results, we can see that a high number of sub-slices is required to exploit the time diversity of the TU6 channel with $80 \mathrm{~Hz}$ of Doppler, whereas the number of sub-slices does not impact the system performance in the case of $10 \mathrm{~Hz}$ of Doppler. The explanation for this is simple; a higher number of sub-slices results in a more uniform distribution over time, achieving a better averaging of fading in fast 

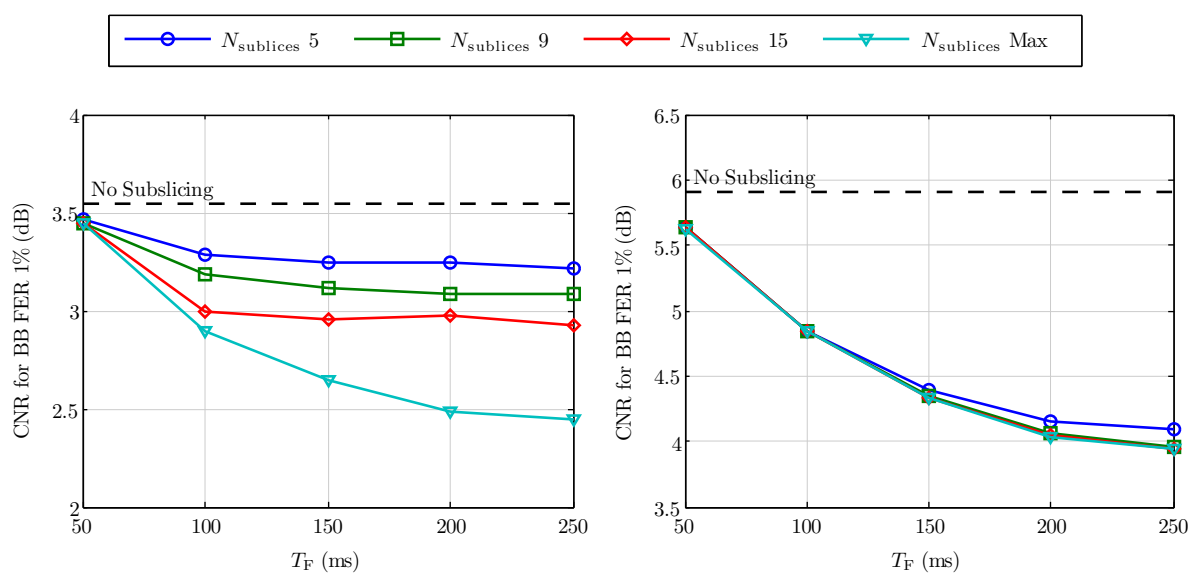

Figure 4.9: Performance of sub-slicing in DVB-T2. TU6 channel with $f_{d}=80 \mathrm{~Hz}$ (left) and $f_{d}=10 \mathrm{~Hz}$ (right). Dashed lines correspond to the case with no sub-slicing.

varying channels. If the channel varies slowly, the interleaving duration rather than the number of sub-slices is more important for time diversity.

The performance of inter-frame interleaving in DVB-T2 is shown in Fig. 4.10. In order to account for the maximum capabilities of the standard, we have selected the maximum number of sub-slices that is possible for each configuration. It should be pointed out that not all configurations of inter-frame interleaving and sub-slicing are allowed in the standard due to undesirable interactions between the time and frequency interleavers. The results in the left side of the figure correspond to interleaving durations between $250 \mathrm{~ms}$ ( 1 frame) and $1 \mathrm{~s}$ (4 frames), whereas the results on the right side correspond to interleaving durations between $250 \mathrm{~ms}$ and a little more than $10 \mathrm{~s}$ (45 frames). We can see that while $250 \mathrm{~ms}$ of TI at the physical layer are generally sufficient to compensate for fast fading, the use of inter-frame interleaving to achieve interleaving durations of several seconds provides gains up to $9 \mathrm{~dB}$ due to better protection against shadowing. Nevertheless, interleaving durations higher than $1 \mathrm{~s}$ are not feasible in DVB-T2 due to limitations in terms of memory and zapping time, and thus, the results presented here have to be taken as an example of the gains that can be achieved with TI at the physical layer.

\section{Signalling Path}

The main objective in this section is to investigate the robustness of the signalling path in mobile channels. As it has been explained before, it is important 

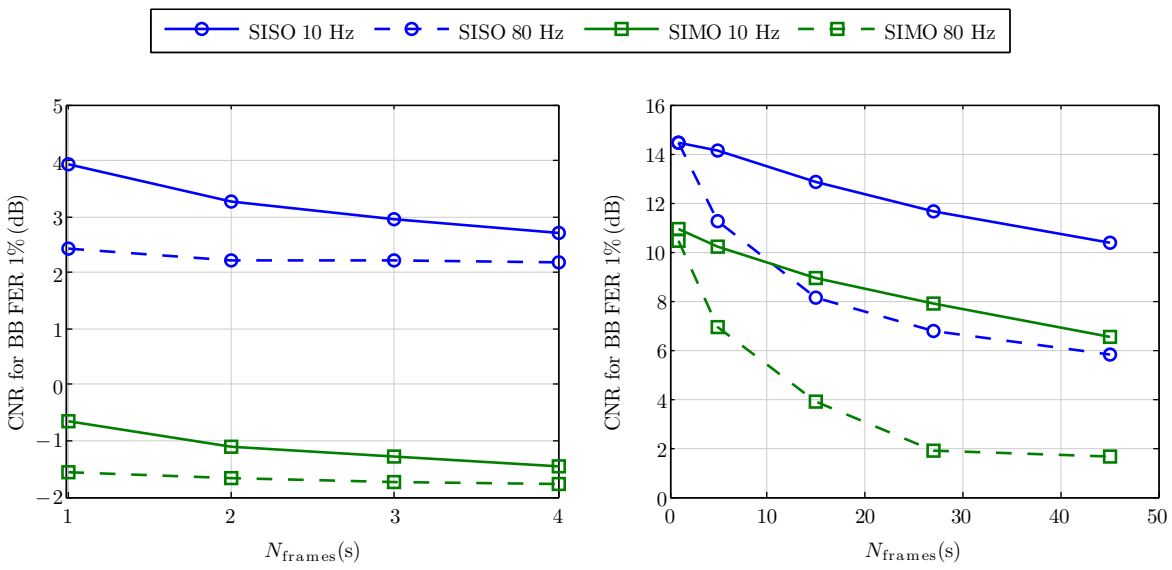

Figure 4.10: Performance of inter-frame interleaving in DVB-T2. On the left, interleaving durations up to $1 \mathrm{~s}$ in the TU6 channel. On the right, interleaving durations up to $10 \mathrm{~s}$ in the TU6 channel with shadowing $\left(\sigma=5.5 \mathrm{~dB}\right.$ and $\left.d_{\mathrm{corr}}=20 \mathrm{~m}\right)$.

that the signalling path is transmitted at least $3 \mathrm{~dB}$ more robust than the data path in order to ensure the reception of services. In Fig. 4.11, we illustrate the capacity curves of the L1 post signalling in the TU6 channel. Each point in the curves corresponds to the four transmission modes that are possible for the L1 post signalling; BPSK 1/2 (0.5 bpc), QPSK 1/2 (1 bpc), 16QAM 1/2 (2 bpc), and 64QAM 1/2 (3 bpc). For the sake of comparison, we have also represented the capacity curves of the data path with $250 \mathrm{~ms}$ of TI. In this case, each point in the curve corresponds to QPSK 1/2 (1 bpc), 16QAM 1/2 (2 bpc), 64QAM 1/2 (3 bpc), and 256QAM 1/2 (4 bpc). By looking at these curves, it is possible to identify for each configuration of MODCOD in the data path, the transmission mode that is necessary in the L1 signalling to achieve a robustness of $3 \mathrm{~dB}$ higher or more. According to the results, the use of repetition is not sufficient to ensure a $3 \mathrm{~dB}$ advantage for the L1 post signalling when the data path is transmitted with QPSK, and in this case, advanced decoding is needed. For the rest of constellations, repetition can provide an adequate robustness. It must be noted however, that advanced decoding allows the L1 post signalling to be transmitted with a higher order constellation (e.g. from BPSK to QPSK) while maintaining the same level of robustness. This reduces the overhead caused by the physical layer signalling, and leaves more capacity for the transmission of data in the P2 symbols. 

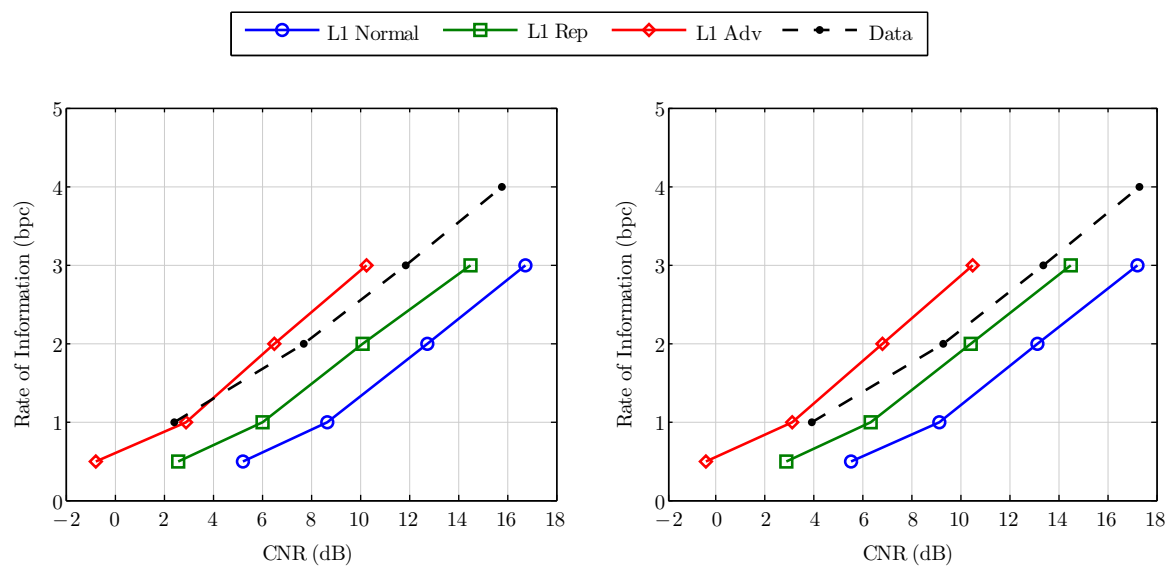

Figure 4.11: Performance of L1 post signalling in DVB-T2. TU6 channel with $f_{d}=80 \mathrm{~Hz}$ (left) and $f_{d}=10 \mathrm{~Hz}$ (right).

\section{Distributed MISO}

In Fig. 4.12, we represent the gain of distributed MISO depending on the delay and the power imbalance that exist between the signals arriving from different transmitters. The simulations have been performed in the SFN TU6 channel assuming an interleaving duration of $250 \mathrm{~ms}$. Each transmitter is modelled as an independent TU6 profile so that the total channel is the sum of all individual and independent channels, each having a unique level and delay. In the figure, we can see that the gain of distributed MISO is largest when there is no delay or power imbalance. The presence of power imbalance is detrimental for diversity, as it reduces the contribution of one transmitter to the received signal. On the other hand, the reception of delayed signals in SFN is similar to the utilization of DD, providing better frequency diversity as a result of increased delay spread. However, this is disadvantageous for distributed MISO, as the rapid variations between subcarriers in the frequency domain can affect the performance of the Alamouti code. Reception at higher Doppler frequencies or the implementation of SIMO in receivers also reduce the improvement of distributed MISO as a result of better diversity in the time and space domain. As it is shown in Fig. 4.12, the gain of distributed MISO in some cases is negligible, and it may actually become negative, resulting in a performance loss compared to SISO.

In Fig. 4.13 we compare the capacity curves of DVB-T2 with and without distributed MISO. The level and delay of each transmitter has been rotated at the beginning of each TI block in order to average the effect of power imbalance 

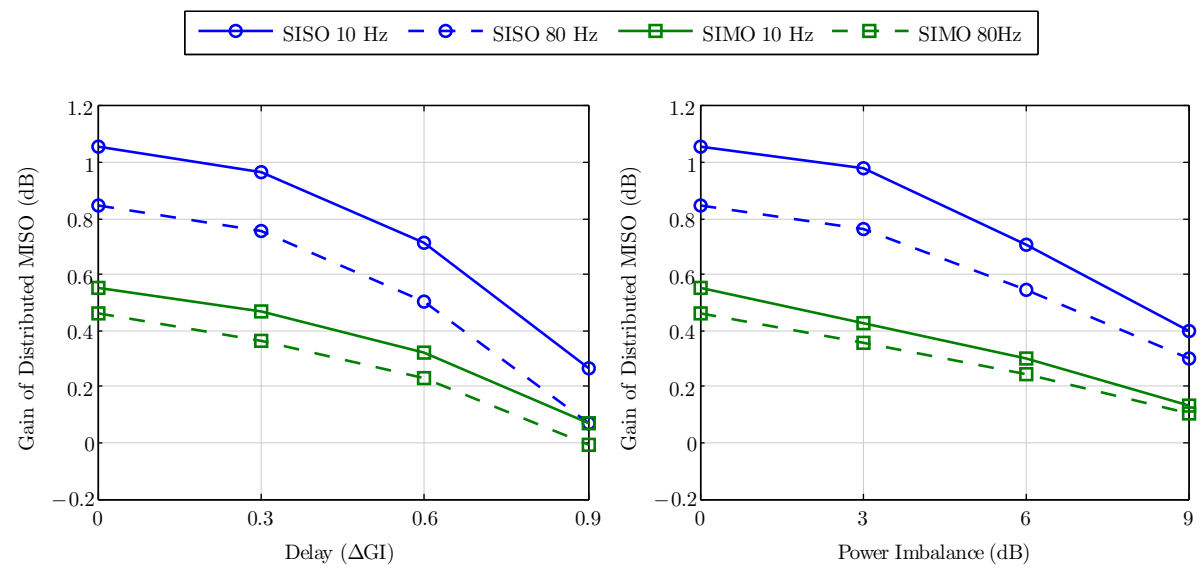

Figure 4.12: Gain of distributed MISO in DVB-T2. SFN TU6 channel with delay (left) and power imbalance (right) between transmitters.

and delay over the simulations. In the left side of the figure, the capacity curves are represented without considering the loss due to the pilot overhead, whereas on the right side, the loss has been taken into account. We have assumed the pilot pattern PP3 for SISO and the pilot pattern PP1 for distributed MISO. Both patterns provide the same quality of channel estimation resulting in an overhead of $4 \%$ and $8 \%$ respectively. From the figure, we can see that the gain of distributed MISO without the pilot overhead is lower than $0.3 \mathrm{~dB}$. With the pilot overhead, distributed MISO reduces the overall spectral efficiency, as in this case, the limited gain in reception robustness does not compensate for the lower capacity.

\section{UL-FEC}

Fig. 4.14 illustrates the performance of AL-FEC in DVB-T2. The results have been obtained with 16QAM 1/2 at the physical layer and different code rates at the application layer. The simulations combine the utilization of intra-frame interleaving and sub-slicing at the physical layer with inter-frame interleaving by means of AL-FEC. The frame length has been set to $250 \mathrm{~ms}$ and thus, the interleaving duration in the figure ranges from $250 \mathrm{~ms}$ (1 frame) up to $10 \mathrm{~s} \mathrm{(40}$ frames). According to the results, the improvement of AL-FEC is similar to the gain obtained with TI at the physical layer (4-9 dB), although in this case, the gain comes at the expense of reduced capacity due to the transmission of AL-FEC parity. If we compare these results with those presented in Fig 4.10, 


\section{○-SFN —- MISO}
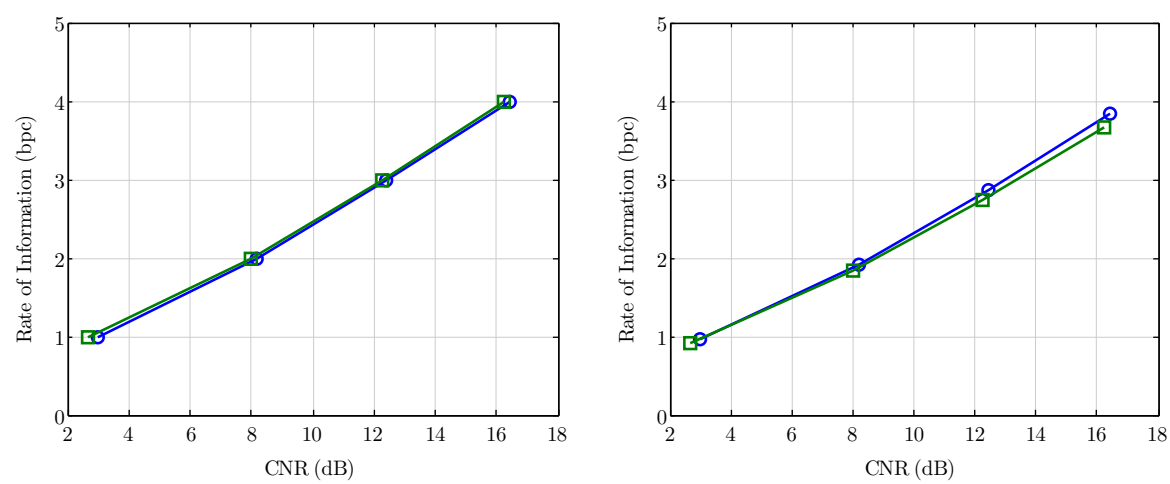

Figure 4.13: Capacity curves of DVB-T2 in the SFN TU6 channel $\left(f_{s}=33 \mathrm{~Hz}\right)$. The delay and power imbalance between transmitters is rotated in each TI block.

which were obtained for QPSK 1/2, we can see that the combination of 16QAM $1 / 2$ at the physical layer with AL-FEC can enable the reception at lower values of $\mathrm{CNR}(<15 \mathrm{~dB})$ for the same or better spectral efficiency ( $>1 \mathrm{bpc})$. This means that, depending on the user velocity, the utilization of AL-FEC in DVB-T2 might be preferable to more robust configuration of MODCOD at the physical layer.

\subsubsection{Network Planning Discussion}

\section{Mobile Reception in DVB-T2 Networks}

Similarly to the investigation presented in Section 4.1.3 for the case of DVB-T, in this section we investigate the provision of mobile services in DVB-T2 networks planned for fixed or portable indoor reception as well as the advantages of diversity techniques in terms of network planning. Mobile services in DVB-T2 networks can be transmitted in dedicated RF channels, or can be multiplexed together with fixed services. The utilization of multiple PLPs or the T2-Lite profile in DVB-T2 allows fixed and mobile services to be multiplexed in time without interfering with each other, such that there is no performance degradation compared to using dedicated RF channels. In DVB-T2 networks planned for fixed reception, using a configuration based on QPSK $1 / 2$ instead of 256QAM 3/5 provides a gain of $15.1 \mathrm{~dB}$ (Rice F1 channel). In networks planned for portable indoor reception, a similar approach would be 


\section{$\multimap$ AL-FEC $7 / 8 \longrightarrow$ ๑一 AL-FEC $5 / 6 \multimap$ AL-FEC $3 / 4 \multimap$ AL-FEC $2 / 3 \multimap$ AL-FEC $1 / 2$}
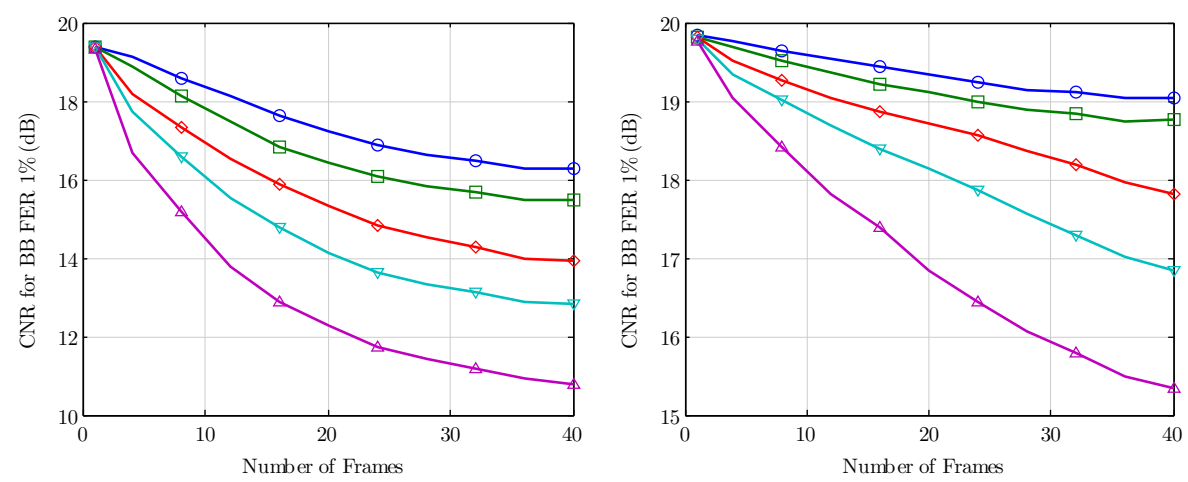

Figure 4.14: Performance of AL-FEC in DVB-T2 according to the number of time-interleaved frames in the TU6 channel with shadowing $\left(f_{d}=80 \mathrm{~Hz}\right.$ left, $f_{d}=10 \mathrm{~Hz}$ right, $\sigma=5.5 \mathrm{~dB}$ and $d_{\text {corr }}=20 \mathrm{~m}$ ).

to use QPSK 1/2 instead of 64QAM 1/2, resulting in a gain of about $9.9 \mathrm{~dB}$ (Rayleigh P1 channel) [42].

In Table 4.5, we compare the link budget of DVB-T2 for fixed, portable, and mobile reception. According to the table, the distance to the link margin of fixed reception is $-47.3 \mathrm{~dB}$ for portable indoor reception, -41.3 for vehicular reception, and -56.8 for handheld indoor reception. Similarly to DVB-T, the utilization of more robust MODCOD together with diversity techniques cannot compensate for such high values, and therefore, mobile services can only be provided in a best effort manner. Table 4.6 shows the coverage probability of vehicular and handheld indoor reception in DVB-T2 networks planned for fixed and portable indoor reception. We can see that the coverage of vehicular and handheld indoor reception in networks planned for fixed reception is below acceptable values (90\% and $70 \%$ respectively). It is important to notice that the height loss shown in Table 4.5 corresponds to the urban scenario, and that lower values are used in the case of suburban and rural scenarios (height loss of $-17.5 \mathrm{~dB}$ and $-16.5 \mathrm{~dB}$ respectively). For example, DVB-T2 networks planned for fixed reception can achieve in rural scenarios a coverage probability as high as $70 \%$ for vehicular reception with SIMO and $250 \mathrm{~ms}$ of TI, whereas the coverage probability in urban scenarios is limited to $22 \%$. 
Table 4.5: Link Budget of DVB-T2 networks in the UHF band

\begin{tabular}{|c|c|c|c|c|}
\hline & Fixed & $\begin{array}{c}\text { Portable } \\
\text { Indoor }\end{array}$ & Vehicular & $\begin{array}{c}\text { Handheld } \\
\text { Indoor }\end{array}$ \\
\hline Multi-path fading loss ${ }^{8}$ & $0.2 \mathrm{~dB}$ & $2 \mathrm{~dB}$ & $7 \mathrm{~dB}$ & $2 \mathrm{~dB}$ \\
\hline Height $\operatorname{loss}^{9}$ & $0 \mathrm{~dB}$ & $23.5 \mathrm{~dB}$ & $23.5 \mathrm{~dB}$ & $23.5 \mathrm{~dB}$ \\
\hline Feeder loss & $4 \mathrm{~dB}$ & $0 \mathrm{~dB}$ & $0 \mathrm{~dB}$ & $0 \mathrm{~dB}$ \\
\hline Building penetration loss & $0 \mathrm{~dB}$ & $11 \mathrm{~dB}$ & $0 \mathrm{~dB}$ & $11 \mathrm{~dB}$ \\
\hline Antenna gain ${ }^{10}$ & $11 \mathrm{~dB}$ & $0 \mathrm{~dB}$ & $0 \mathrm{~dB}$ & $-9.5 \mathrm{~dB}$ \\
\hline Location correction factor ${ }^{11}$ & $9 \mathrm{~dB}$ & $13 \mathrm{~dB}$ & $13 \mathrm{~dB}$ & $13 \mathrm{~dB}$ \\
\hline Link margin total & $2.6 \mathrm{~dB}$ & $49.9 \mathrm{~dB}$ & $41.5 \mathrm{~dB}$ & $59.4 \mathrm{~dB}$ \\
\hline Distance to fixed & - & $-47.3 \mathrm{~dB}$ & $-41.3 \mathrm{~dB}$ & $-56.8 \mathrm{~dB}$ \\
\hline Distance to portable indoor & - & - & $6 \mathrm{~dB}$ & $-9.5 \mathrm{~dB}$ \\
\hline
\end{tabular}

As can be seen in Table 4.6, networks planned for portable indoor reception provide full coverage for vehicular reception and achieve good coverage for handheld indoor reception. In this case, diversity techniques can be used to increase the spectral efficiency of mobile services targeting vehicular receivers. For example, a configuration based on 256QAM 3/5 provides good coverage of vehicular services with a single receive antenna. With two receive antennas, it is possible to use a configuration based on 256QAM 5/6 with the same coverage probability while achieving a $39 \%$ increase in terms of spectral efficiency. Alternatively, the use of diversity techniques together with the lower code rates included in T2-Lite $(1 / 3$ and $2 / 5)$ can extend the coverage of handheld indoor reception in scenarios with severe penetration losses.

In Table 4.7, we show the coverage extension of diversity techniques according to the methodology presented in Section 4.1.3. The results reveal that while the improvement of SIMO is the same as in DVB-T, the potential gain of time diversity in DVB-T2 is higher due to the incorporation of TI at the physical layer. In this manner, it is possible to combine the protection of PL-FEC against fast fading, with the additional robustness against shadowing given by

\footnotetext{
${ }^{8}$ Compared to AWGN according to the values presented in [42]. The MODCOD is assumed to be QPSK 1/2 in portable indoor, vehicular, and handheld indoor reception, and 256QAM $3 / 5$ in fixed reception

${ }^{9}$ Height loss corresponds to the urban scenario.

${ }^{10}$ Antenna gain relative to half wave dipole.

${ }^{11}$ Location correction factor corresponds to good coverage quality (i.e. $95 \%$ in fixed, portable indoor, and handheld indoor, and 99\% in vehicular).
} 
CHAPTER 4. COMBINED DIVERSITY IN CURRENT SYSTEMS

Table 4.6: Coverage probability of mobile services in DVB-T2 networks

\begin{tabular}{|c|c|c|c|c|}
\hline \multirow{2}{*}{$\begin{array}{l}\text { Diversity } \\
\text { Techniques }\end{array}$} & \multicolumn{2}{|c|}{$\begin{array}{l}\text { Network planned for } \\
\text { fixed reception }^{12}\end{array}$} & \multicolumn{2}{|c|}{$\begin{array}{l}\text { Network planned for } \\
\text { portable indoor }^{13}\end{array}$} \\
\hline & Vehicular & $\begin{array}{l}\text { Handheld } \\
\text { Indoor }\end{array}$ & Vehicular & $\begin{array}{l}\text { Handheld } \\
\text { Indoor }\end{array}$ \\
\hline- & $1 \%$ & $0 \%$ & $100 \%$ & $95 \%$ \\
\hline SIMO & $10 \%$ & $0 \%$ & $100 \%$ & - \\
\hline PL-FEC 1/2 $250 \mathrm{~ms}$ & $1 \%-7 \%$ & $0 \%$ & $100 \%$ & $95 \%-97 \%$ \\
\hline $\begin{array}{c}\text { PL-FEC } 1 / 2250 \mathrm{~ms}+ \\
\text { UL-FEC } 1 / 210 \mathrm{~s}\end{array}$ & $1 \%-56 \%$ & $0 \%$ & $100 \%$ & $95 \%-99 \%$ \\
\hline $\begin{array}{c}\text { SIMO + } \\
\text { PL-FEC } 1 / 2250 \mathrm{~ms}\end{array}$ & $10 \%-22 \%$ & $0 \%$ & $100 \%$ & - \\
\hline $\begin{array}{c}\text { SIMO + } \\
\text { PL-FEC } 1 / 2250 \mathrm{~ms} \\
\text { UL-FEC } 1 / 210 \mathrm{~s}\end{array}$ & $10 \%-81 \%$ & $0 \%$ & $100 \%$ & - \\
\hline
\end{tabular}

UL-FEC techniques. It must be noted that although the values listed in the table show very large gains for UL-FEC and PL-FEC, these depend on the velocity and the route of the user across the service area, and therefore, they must be interpreted as the highest gains that are possible in mobile reception conditions.

\section{Multiplexing of Mobile Services in DVB-T2 Networks}

In addition to the previous considerations in terms of link budget, mobile reception is challenged by the presence of ICI. This is caused by the Doppler spread and limits the maximum velocity for which reception is possible in the service area. DVB-T2 networks planned for fixed reception employ large FFTs and sparse pilot patterns in order to achieve better capacity with sufficient robustness for rooftop antennas. In contrast, reception at high velocities requires the utilization of smaller FFTs and more dense pilot patterns to follow the rapid variations in the time domain.

\footnotetext{
${ }^{12}$ The values have been obtained assuming 256QAM 3/5 for fixed reception and QPSK 1/2 for mobile and handheld indoor reception.

${ }^{13}$ The values have been obtained assuming 64QAM 3/5 for fixed reception and QPSK 1/2 for mobile and handheld indoor reception.
} 
Table 4.7: Coverage extension of mobile services in DVB-T2 networks ${ }^{14}$

\begin{tabular}{c||c|c}
\hline $\begin{array}{c}\text { Diversity } \\
\text { Technique }\end{array}$ & $\begin{array}{c}\text { Link Margin } \\
\text { Gain }\end{array}$ & $\begin{array}{c}\text { Coverage } \\
\text { Extension }\end{array}$ \\
\hline \hline SIMO & $6 \mathrm{~dB}$ & $120 \%$ \\
\hline PL-FEC $250 \mathrm{~ms}$ & $0 \mathrm{~dB}-5 \mathrm{~dB}$ & $0 \%-93 \%$ \\
\hline $\begin{array}{c}\text { PL-FEC } 250 \mathrm{~ms}+ \\
\text { UL-FEC } 1 / 210 \mathrm{~s}\end{array}$ & $0 \mathrm{~dB}-14 \mathrm{~dB}$ & $0 \%-531 \%$ \\
\hline SIMO + PL-FEC $250 \mathrm{~ms}$ & $6 \mathrm{~dB}-9 \mathrm{~dB}$ & $120 \%-227 \%$ \\
\hline SIMO + PL-FEC $250 \mathrm{~ms}+$ & $6 \mathrm{~dB}-18 \mathrm{~dB}$ & $120 \%-968 \%$ \\
UL-FEC $1 / 210 \mathrm{~s}$ & & \\
\hline
\end{tabular}

Network operators can decide the use of multiple PLPs or the T2-Lite profile in order to multiplex fixed and mobile services in the same RF channels. In the case of multiple PLPs, the FFT mode and the pilot pattern must be common for all the PLPs transmitted in the same multiplex, whereas the T2Lite profile allows alternating different configurations in the time domain by means of FEF parts. For the discussion regarding the utilization of multiple PLPs and T2-Lite, we compare two different DVB-T2 configurations in terms of spectral efficiency. The first configuration is based on multiple PLPs and employs a robust combination of FFT size and pilot pattern for the entire multiplex. In contrast, the second configuration is based on T2-Lite and alternates between different combinations for the transmission of fixed and mobile services. The transmission parameters of the two configurations are listed in Table 4.8. We can see that both configuration provide the same robustness for fixed and mobile services and also the same duration of guard interval.

The total spectral efficiency in one RF channel that combines fixed and mobile services by means of multiple PLPs, $S_{\mathrm{PLP}}$, can be approximated by:

$$
S_{\mathrm{PLP}} \approx \frac{N_{d}}{N_{c}} \cdot(1-G) \cdot\left(T_{f} \cdot B_{f}+T_{m} \cdot B_{m}\right),
$$

where $N_{c}$ is the total number of subcarriers per OFDM symbol, $N_{d}$ is the number of subcarriers with data (non-pilot) information per OFDM symbol, $G$ is the fraction of guard interval, $B_{f}$ is the number of bits per cell in the case of fixed services, $B_{m}$ is the number of bits per cell in the case of mobile services,

\footnotetext{
${ }^{14}$ The gains of SIMO correspond to a implementation based on two co-polar antennas with sufficient separation between the antennas. The gains of UL-FEC and PL-FEC depend on the user velocity and the route across the service area. The highest value corresponds to the case when the user is moving at a constant velocity of $144 \mathrm{~km} / \mathrm{h}$.
} 
CHAPTER 4. COMBINED DIVERSITY IN CURRENT SYSTEMS

Table 4.8: Configuration for the combination of fixed and mobile services in DVB-T2 networks.

\begin{tabular}{c|c|c|c|c}
\hline \multirow{2}{*}{$\begin{array}{c}\text { Configuration } \\
\text { Parameter }\end{array}$} & \multicolumn{2}{|c|}{$\begin{array}{r}\text { Configuration based on } \\
\text { multiple PLPs }\end{array}$} & \multicolumn{2}{|c}{$\begin{array}{r}\text { Configuration based on } \\
\text { T2-Lite }\end{array}$} \\
\cline { 2 - 5 } & Fixed & Mobile & Fixed & Mobile \\
\hline \hline FFT size & $8 \mathrm{~K}$ Ext. & $8 \mathrm{~K}$ Ext. & $32 \mathrm{~K}$ Ext. & $8 \mathrm{~K}$ Ext. \\
\hline Guard interval & $1 / 4$ & $1 / 4$ & $1 / 16$ & $1 / 4$ \\
\hline Pilot pattern & $\mathrm{PP} 1$ & $\mathrm{PP} 1$ & $\mathrm{PP} 4$ & $\mathrm{PP} 1$ \\
\hline Constellation & $256 \mathrm{QAM}$ & $\mathrm{QPSK}$ & $256 \mathrm{QAM}$ & $\mathrm{QPSK}$ \\
\hline Code rate & $3 / 5$ & $1 / 2$ & $3 / 5$ & $1 / 2$ \\
\hline
\end{tabular}

$T_{f}$ is the portion of time dedicated to the transmission of fixed services, and $T_{m}$ is the portion of time dedicated to the transmission of mobile services. On the contrary, if the T2-Lite profile is used instead, the total spectral efficiency, $S_{\text {Lite }}$, can be computed as:

$$
S_{\text {Lite }} \approx \frac{N_{d}^{f}}{N_{c}^{f}} \cdot\left(1-G_{f}\right) \cdot T_{f} \cdot B_{f}+\frac{N_{d}^{m}}{N_{c}^{m}} \cdot\left(1-G_{m}\right) \cdot T_{m} \cdot B_{m},
$$

where $N_{c}^{f}, N_{d}^{f}, G^{f}, N_{c}^{m}, N_{d}^{m}$, and $G^{m}$ are respectively the number of subcarriers per OFDM symbol, the number of subcarriers with data information per OFDM symbol, and the guard interval in the case of fixed and mobile services.

It is important to note that although we have not included the percentage of time that is needed for the transmission of L1-signalling, this represents less than $1 \%$ of the total transmission time, and thus, it has a minimal impact in the calculations. Assuming that the $80 \%$ of the transmission time is reserved to the transmission of fixed services $\left(T_{f}=0.8\right)$ and that the $20 \%$ is dedicated to the transmission of mobile services $\left(T_{m}=0.2\right)$, the total spectral efficiency for the configuration using multiple PLPs is approximately $2.3 \mathrm{bpc}$, whereas it is approximately $3 \mathrm{bpc}$ for the configuration using T2-Lite. This represents an advantage in the order of $30 \%$ for T2-Lite.

In Fig 4.15 we illustrate the overall spectral efficiency for DVB-T2 networks using multiple PLPs or T2-Lite according to the percentage of time that is dedicated to the transmission of mobile services. We can see that the advantage of T2-Lite diminishes when the transmission of mobile services occupies a higher percentage of the transmission time. This continues up to the point at which there is no time reserved for the transmission of fixed services $\left(T_{m}=1\right)$, and the T2-Lite profile provides no gain. 


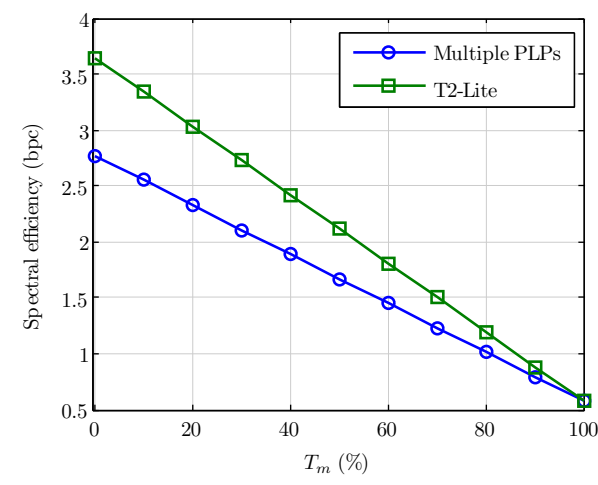

Figure 4.15: Spectral efficiency of DVB-T2 networks when using multiple PLPs or the T2-Lite profile for the combination of fixed and mobile services.

\subsection{Performance Comparison between DVB-T and DVB-T2}

The efficiency of DVB-T2 for high throughput has been improved by means of larger FFT modes (16K and $32 \mathrm{~K})$, extended carrier modes, optimized pilot patterns, and reduced L1 signalling. For the same separation between transmitters, larger FFT modes reduce the percentage of time that is dedicated to the transmission of the guard interval at the expense of lower Doppler performance. Extended carrier modes increase the number of data carriers with FFT modes $32 \mathrm{~K}, 16 \mathrm{~K}$, and $8 \mathrm{~K}$, whereas optimized pilot patterns minimize the number of pilots that must be transmitted for channel estimation according to the maximum delay spread and Doppler that is expected in the channel. The combination of these features provides a capacity increase of around $30 \%$ compared to DVB-T [42].

On the other hand, the reception robustness in DVB-T2 has been improved with better FEC codes, rotated constellations, and the incorporation of TI at the physical layer. In particular, the advantage of DVB-T2 over its predecessor is about $2.5 \mathrm{~dB}$ in AWGN [107] due to the incorporation of LDPC codes, while the implementation of TI at the physical layer provides additional gains in mobile scenarios. In Fig. 4.16, we compare the capacity curves of DVB-T and DVB-T2 in the TU6 channel. In the case of DVB-T, we show the curves corresponding to the case with and without AL-FEC (250 ms), whereas in DVB-T2, we show the curves corresponding to $250 \mathrm{~ms}$ of TI at the physical layer. For the sake of comparison, we also represent the outage capacity of the 

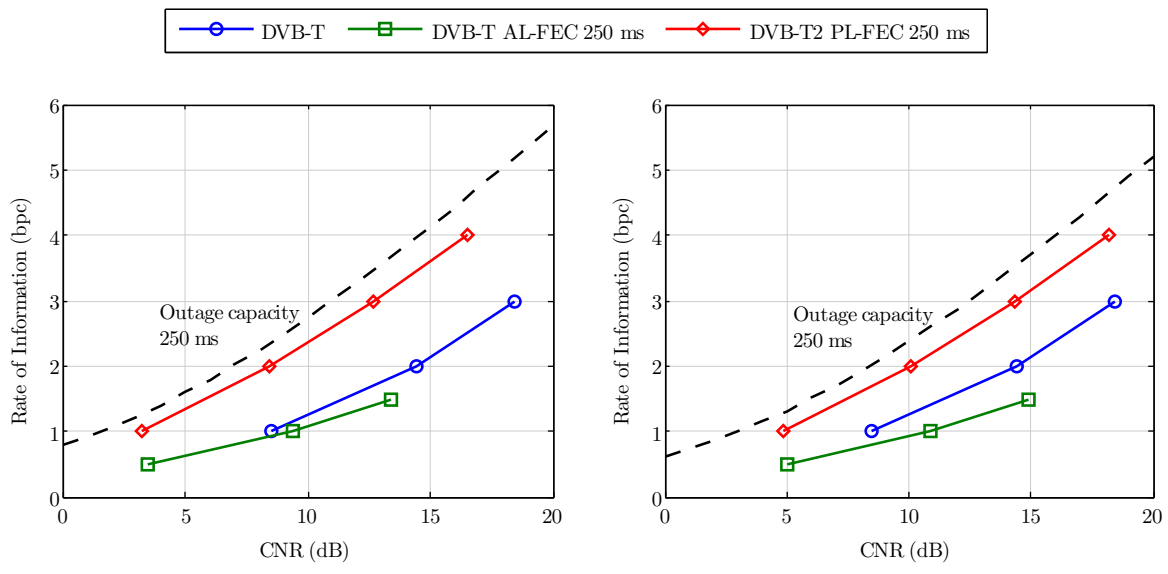

Figure 4.16: Comparison between the capacity curves of DVB-T and DVB-T2. TU6 channel with $f_{d}=80 \mathrm{~Hz}$ (left) and $f_{d}=10 \mathrm{~Hz}$ (right). Dashed curves correspond to the outage capacity of the channel with $250 \mathrm{~ms}$ of TI.

channel according to the methodology described in Section 3.2.2. In the figure, DVB-T2 outperforms DVB-T in about 4-6 dB for the same spectral efficiency. Despite the fact that AL-FEC enables the reception of DVB-T services at CNR values as low as with the most robust modes in DVB-T2, it does not improve the overall spectral efficiency due to the transmission of additional parity data.

The improved reception robustness of DVB-T2 can be translated into a higher capacity for the transmission of mobile services by using higher code rates and larger constellations. The simulation results show that the potential capacity increase of DVB-T2 over DVB-T as a result of better robustness at the physical layer is around $100 \%$ for low CNR values $(<10 \mathrm{~dB})$, and around $75 \%$ for high $\mathrm{CNR}$ values $(>10 \mathrm{~dB})$

\subsection{Conclusions}

In this chapter, we have investigated the combined use of diversity in the time and space domains to improve the reception of mobile services in DVB-T, DVB-H, and DVB-T2 systems. The investigations were aimed at the simultaneous provision of fixed and mobile services in DTT networks, which is considered as key for the start-up of commercial mobile services.

We have shown that the utilization of AL-FEC in DVB-T outperforms MPE-FEC in DVB-H as a result of continuous transmission and longer TI. 
AL-FEC can be incorporated in a backwards compatible way with legacy receivers, can be applied on a service basis, and with Raptor codes, it allows the FEC decoding to be done in generic software processors without the need of any upgrades in hardware. According to the simulation results, the use of AL-FEC with interleaving durations higher than $1 \mathrm{~s}$ achieves important gains against shadowing $(\leq 10 \mathrm{~dB})$. However, the gain of AL-FEC against fast fading is much more limited $(\leq 5 \mathrm{~dB})$, and for interleaving durations lower than $1 \mathrm{~s}$, it is preferable to employ a more robust configuration at the physical layer instead of using AL-FEC. In DVB-H, inter-burst protection can be achieved by means of MPE-iFEC with similar results to AL-FEC in DVB-T. From the two mappings defined in MPE-iFEC, the configuration based on Raptor codes outperforms the one based on RS in less than $1 \mathrm{~dB}$. In the space domain, the utilization of SIMO achieves a $6 \mathrm{~dB}$ gain if UL-FEC techniques are not used for time diversity. Since both AL-FEC and SIMO exploit the diversity caused by multipath propagation, the improvement of using both AL-FEC and SIMO is lower than the individual sum of gains.

In DVB-T2, we have characterized the trade-offs that are possible in terms of robustness (diversity), data rate, power saving, and zapping time. It has been shown that although the TI included in DVB-T2 can be extended across several seconds, the maximum interleaving duration in practice is limited by the service data rate and the zapping time. This is motivated by the amount of memory available in receivers and the lack of fast zapping support. The simulation results show that the performance of TI against fast fading depends not only on the interleaving duration but also on the number of sub-slices per T2 frame. For high values of Doppler, a large number of sub-slices is needed to exploit the time diversity of the channel, whereas for low values of Doppler, the interleaving duration is more important. Our investigations reveal that intra-frame interleaving $\left(T_{\mathrm{int}} \leq 250 \mathrm{~ms}\right)$ is generally sufficient to compensate the fast fading, whereas interleaving durations up to $10 \mathrm{~s}$ by means of inter-frame interleaving can provide gains up to $9 \mathrm{~dB}$ as a result of better protection against shadowing. The simulations results show that the gains of long TI with AL-FEC are similar to those obtained at the physical layer, but at the expense of reduced capacity due to the transmission of AL-FEC parity. The robustness of the L1 signalling information in mobile channels has been taken into account when evaluating the performance of DVB-T2. Since the signalling information is transmitted in the first symbols of the T2 frames without TI, advanced decoding techniques have to be used so that the robustness of the signalling is $3 \mathrm{~dB}$ higher than the data path for the more robust configurations. In the space domain, the utilization of SIMO achieves similar gains as in DVB-T, i.e. between 4-6 dB depending on TI. In contrast, the improvement of distributed MISO in mobile channels is much more limited 
$(<1 \mathrm{~dB})$, being conditioned by the power imbalance and the delay between the signals from different transmitters. Overall, distributed MISO lowers the spectral efficiency if the transmission of pilots is taken into account, and in some cases, it may also degrade the system performance in terms of CNR.

The investigations performed in terms of network planning reveal that mobile reception of DVB-T and DVB-T2 services with good coverage levels is not possible in networks planned for fixed reception, even with more robust configurations of MODCOD and the utilization of diversity techniques. On the contrary, networks planned for portable indoor reception achieve full coverage (i.e. $100 \%$ ) for vehicular reception and close to good coverage levels (i.e. 95\%) for handheld indoor reception without the help of diversity techniques. In this case, the incorporation of diversity techniques can be aimed to improve the spectral efficiency of services targeting vehicular receivers and also to improve the coverage of handheld indoor reception in scenarios with severe penetration losses. 


\section{Chapter 5}

\section{Combined Diversity in the Next Generation of Terrestrial Mobile Broadcasting Systems}

The adoption of robust FEC codes such as turbo codes in DVB-SH or LDPC codes in DVB-T2, allows moderns broadcasting systems to achieve a performance close to the channel capacity in AWGN. As a result, the use of diversity in the time, frequency, and space domains is necessary in the next generation of mobile broadcasting systems to achieve a significant performance increase compared to the current generation. In this sense, DVB-NGH will be the first mobile broadcasting system to include the use of diversity in the three domains by incorporating at the physical layer long TI, TFS, and cross-polar MIMO.

DVB-NGH adopts a combination of intra-frame BI in the order of hundreds of milliseconds and inter-frame CI up to several seconds for the provision of TI at the physical layer. In the hybrid terrestrial-satellite profiles, the standard has also included UL interleaving in order to reduce the zapping time at the expense of reception robustness (time diversity). In this chapter, we evaluate the performance of long TI in DVB-NGH, and compare the solution adopted in the standard with BB-iFEC, a split FEC approach that has been specifically designed to achieve a good compromise between time diversity and zapping time.

The incorporation of TFS in DVB-NGH is expected to provide a significant advantage by means of improved StatMux and better frequency diversity. 


\section{CHAPTER 5. COMBINED DIVERSITY IN NEXT GENERATION SYSTEMS}

While the potential gains of TFS as a result of better frequency diversity were first computed in Chapter 3 from an information-theoretic point of view, here, we evaluate the gains achieved in DVB-NGH systems with physical layer simulations.

DVB-NGH has adopted cross-polar MIMO as a manner to achieve space diversity and overcome the Shannon limit of single antenna communications. In this chapter, we assess the performance of MIMO in DVB-NGH systems while taking into account two important aspects that affect the performance of MIMO techniques: the amount of capacity that is needed for pilot information, and the error-correction capabilities of FEC codes.

In addition to the investigation of each type of diversity in DVB-NGH, we also consider the combined use of diversity, and particularly, how the utilization of TFS and MIMO affects the performance gains of long TI. In parallel to long TI, TFS, and MIMO, we investigate the gains that can be obtained with rotated constellations in DVB-NGH. The standard inherits the same 2DRC from DVB-T2 and includes 4DRC for certain configurations. Moreover, a new component interleaver has been optimized for the utilization of rotated constellations with long TI and TFS. The main focus in this case is to study the gains that can be achieved by combining rotated constellations with long TI and TFS in DVB-NGH systems.

The rest of the Chapter is structured as follows. In Section 5.1, Section 5.2, and Section 5.3, we evaluate the use of long TI, TFS, and cross-polar MIMO in a separate manner. In Section 5.4, we investigate the combined use of long TI with TFS and MIMO. In Section 5.5, we focus on rotated constellations. Finally, we give some concluding remarks in Section 5.6.

\subsection{Time Diversity}

\subsubsection{Analysis of Time Interleaving in DVB-NGH}

DVB-NGH introduces two main modifications in the TI compared to DVB-T2: inter-frame CI and adaptive cell quantization for QPSK and 16QAM constellations. Both modifications improve the memory efficiency of the interleaving process, being able to provide longer interleaving durations for a given service data rate, or support higher service data rates for a given interleaving duration. CI also lowers the end-to-end delay and the zapping time compared to DVB-T2. Moreover, for the hybrid terrestrial-satellite profiles, it is possible to use UL interleaving in order to achieve long TI with reduced zapping times.

This section presents an investigation regarding the trade-offs that are related to the use of TI in DVB-NGH, including time diversity, data rate, and latency. Due to the fact that the capabilities of the new standard in terms 
of power saving are the same as in DVB-T2, the reader should refer to Section 4.2.1 for the discussion regarding the trade-off between time diversity and power saving.

\section{Trade-off between Time Diversity and Data Rate}

As in DVB-T2, the use of TI in DVB-NGH is limited by the service data rate, the amount of TI memory in receivers, and the configuration of MODCOD. In particular, the maximum interleaving duration in DVB-NGH can be computed as:

$$
T_{\max } \approx \frac{2 \times\left\lfloor\frac{M_{\mathrm{TI}} \times \eta_{\mathrm{MOD}}}{N_{\mathrm{LDPC}}}\right\rfloor \times\left(K_{\mathrm{BCH}}-80\right)}{R_{\mathrm{PLP}}},
$$

where $M_{\mathrm{TI}}$ is the number of cells that can be stored in the TI memory, $\eta_{\mathrm{MOD}}$ is the number of bits per constellation symbol (e.g., 2 bits for QPSK), $N_{\text {LDPC }}$ is the number of encoded bits per LDPC codeword (16200 bits), $K_{\mathrm{BCH}}$ is the number of source bits per BCH codeword (e.g. 8472 bits for code rate 8/15), and $R_{\mathrm{PLP}}$ is the data rate of any PLP carried in the NGH signal (in bps). Compared to DVB-T2, in which the number of cells that can be fit in the TI memory is the same for every configuration of MODCOD, the utilization of adaptive cell quantization in DVB-NGH doubles the number of cells that can be stored for low order constellations. As a result, the value of $M_{\mathrm{TI}}$ in terrestrial receivers is $2^{19}$ cells for QPSK and 16QAM, and $2^{18}$ cells for the rest of constellations. In hybrid receivers, the value of $M_{\mathrm{TI}}$ is $2^{22}$ cells for QPSK and 16QAM.

In Fig. 5.1, we compare the maximum interleaving duration of T2-Lite and DVB-NGH for terrestrial and hybrid receivers. The results have been obtained for QPSK, as this is the most limiting constellation. According to Fig. 5.1, interleaving durations higher than $1 \mathrm{~s}$ are generally not possible in T2-Lite receivers, whereas interleaving durations of several seconds can be achieved in terrestrial DVB-NGH receivers for data rates below $1 \mathrm{Mbps}$. The combination of CI and adaptive cell quantization allows terrestrial DVB-NGH receivers to support interleaving durations four times longer than T2-Lite receivers with the same TI memory. On the other hand, the incorporation of an external TI memory in hybrid DVB-NGH receivers can enable interleaving durations higher than $10 \mathrm{~s}$ with low data rates $(<1 \mathrm{Mbps})$, while several seconds are still possible with data rates up to $4 \mathrm{Mbps}$. 

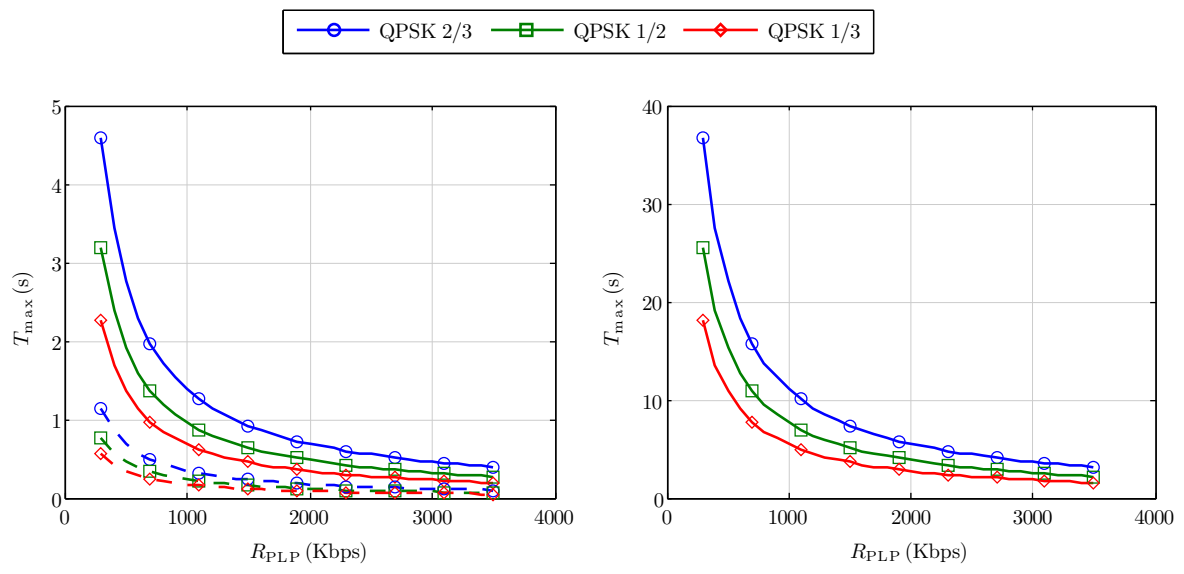

Figure 5.1: Maximum interleaving duration in DVB-NGH according to the service data rate with terrestrial (left) and hybrid receivers (right). Dashed lines correspond to T2-Lite. Code rate $1 / 2$ corresponds with $4 / 9$ in T2-Lite and with $7 / 15$ in DVB-NGH.

\section{Trade-off between Time Diversity and Latency}

In DVB-T2, receivers have to wait until the complete reception of one TI block before they can start decoding the service. As a result, the zapping time is about one and a half times the interleaving duration on average. On the contrary, due to the incorporation of CI, the zapping time in DVB-NGH is approximately equal to the interleaving duration, or even lower with fast zapping. It should be noted that this reduction does not apply to the case when only intra-frame BI is used.

The zapping time in DVB-NGH is given by:

$$
T_{\text {change }}=T_{\text {wait }}+T_{\text {dec }}
$$

where $T_{\text {wait }}$ is the average period of time until the arrival of the first frame with information, and $T_{\mathrm{dec}}$ is the time that passes between the reception of the first frame with information and the instant in which the receiver starts decoding the service. Depending on the amount of information that is available for decoding after zapping, we distinguish between early decoding and late decoding. Early decoding enables fast zapping with long TI and refers to the possibility to start decoding the service before the reception of all the data and parity information. Late decoding provides the highest robustness and refers to the decoding of codewords when all the information is available at the receiver side. For more 


\subsection{Time Diversity}

\section{$\longrightarrow F_{\text {interval }} 1 \longrightarrow F_{\text {interval }} 5 \stackrel{\diamond}{\longrightarrow} F_{\text {interval }} 10$}
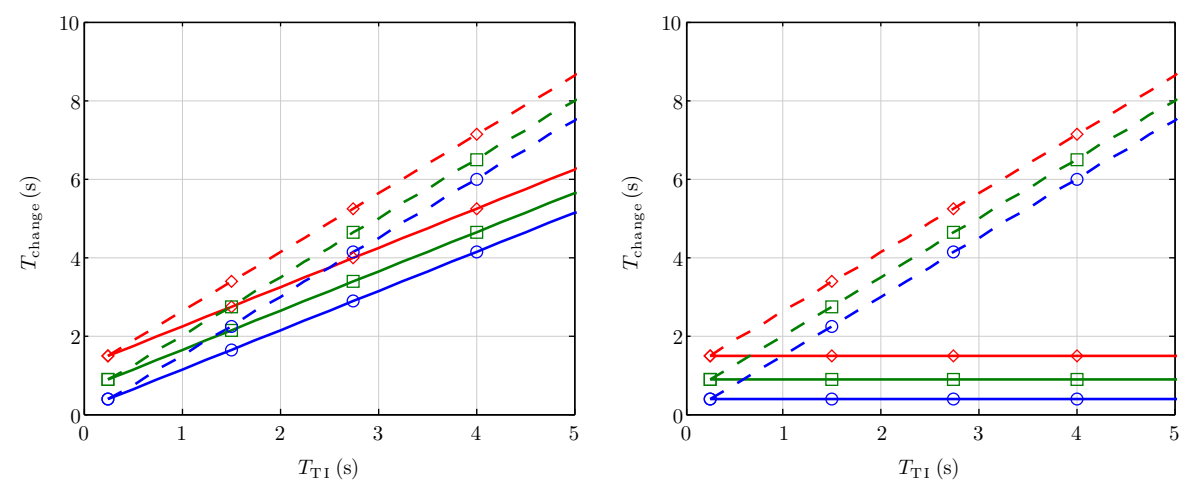

Figure 5.2: Average zapping time in DVB-NGH according to the interleaving duration for the case of late decoding (left) and early decoding (right). Dashed lines correspond to DVB-T2.

details regarding the distinction between early and late decoding, the reader should refer to Section 2.4.

With the same assumptions as in Section 4.2.1, the value of $T_{\text {wait }}$ can be computed as:

$$
T_{\text {wait }} \approx \frac{T_{\mathrm{F}} \times F_{\text {interval }}}{2},
$$

where $T_{\mathrm{F}}$ is the length of the T2 frame and $F_{\text {interval }}$ is the frame interval.

On the other hand, $T_{\mathrm{dec}}$ is lowest in the case of early decoding after the reception of the first frame with information, and it is highest in the case of late decoding. For the case of early decoding, the value of $T_{\mathrm{dec}}$ is approximately:

$$
T_{\text {dec }}=T_{\text {early }} \approx T_{\mathrm{F}},
$$

whereas for late decoding, the value of $T_{\mathrm{dec}}$ can be approximated as:

$$
T_{\text {dec }}=T_{\text {late }} \approx T_{\mathrm{F}} \times\left(F_{\text {interval }} \times\left(N_{\text {frames }}-1\right)+1\right) .
$$

In Fig. 5.2, we compare the average zapping time in DVB-T2 and DVB-NGH systems for early decoding and late decoding. In the left side of the figure, we can see that the zapping time of DVB-NGH in late decoding is approximately a $33 \%$ lower than in DVB-T2 due to the utilization of CI. In spite of this, interleaving durations of several seconds are not possible with tolerable zapping times (i.e. below $2 \mathrm{~s}$ ). On the contrary, the zapping time of DVB-NGH in 


\section{CHAPTER 5. COMBINED DIVERSITY IN NEXT GENERATION SYSTEMS}

Table 5.1: Equivalent code rate in early decoding with different UL interleaving configurations

\begin{tabular}{c||c|c|c|c}
\hline \hline Information carried in the late part & $50 \%$ & $45 \%$ & $40 \%$ & $30 \%$ \\
\hline Overall code rate $1 / 3$ & $2 / 3$ & $11 / 15$ & $5 / 6$ & - \\
\hline Overall code rate $1 / 5$ & $2 / 5$ & $4 / 9$ & $1 / 2$ & $2 / 3$ \\
\hline \hline
\end{tabular}

the case of early decoding does not depend on the interleaving duration and varies only with the frame interval. Zapping times below $2 \mathrm{~s}$ are possible with frame intervals as high as 10 (i.e. power saving 90\%). It should be noted that early decoding after the reception of the first frame can only be achieved in good reception conditions, in which the FEC code can compensante for a large percentage of erasures within the codewords. Otherwise, the zapping time increases up to the point in which the receiver has enough information to start decoding the codewords.

In the hybrid terrestrial-satellite profiles, it is possible to employ UL configurations of inter-frame CI to improve early decoding and facilitate fast zapping. In this case, a large portion of information from the codewords is transmitted in a late frame with a duration of typically hundreds of milliseconds, so that the receiver can start decoding the service immediately after the reception of the late frame. However, this results in a non-uniform interleaving of information over time, which might reduce the performance of late decoding in mobile channels. The trade-off between early and late decoding is therefore determined by the percentage of information that is carried in the late part.

In early decoding, the missing parts of the codewords can be considered as erasures of LLRs at the input of the LDPC decoder. When UL interleaving is used, the information that is available for LDPC decoding after fast zapping corresponds to the late part, whereas the erasures correspond to the bits that are transmitted in the uniform part. The larger the percentage of information that is carried in the late part, the lower the number of erasures that have to be corrected by the LDPC code immediately after the reception of the late frame. The percentage of erasures after receiving the first information frame is given by:

$$
E=\left(100-I_{\text {late }}\right)
$$

where $I_{\text {late }}$ is the percentage of information carried in the late part. Although good punctured LDPC codes with a performance close to the mother code are possible in AWGN [108-110], this depends on the code structure and the puncturing pattern. In the case of DVB-NGH, the erasures are distributed in a pseudorandom manner by the cell deinterleaving that is placed after the time 


\subsection{Time Diversity}

deinterleaver. Since the puncturing pattern is not optimized to the code structure, some performance loss can be expected in early decoding when compared to the equivalent non-punctured LDPC code.

Table 5.1 shows the correspondence between the percentage of information that is carried in the late part, and the equivalent code rate for early decoding after receiving the first information frame, which can be computed as:

$$
R_{\text {early }}=\frac{R_{\text {late }}}{\left(100-I_{\text {late }}\right) / 100},
$$

where $R_{\text {early }}$ and $R_{\text {late }}$ denote the code rate for early and late decoding respectively. Typical configurations for long TI generally employ very robust code rates like $1 / 3$ or $1 / 5$ in order to enable the reception at low CNR. The value corresponding to $30 \%$ with code rate $1 / 3$ is not listed in the table, as this code rate is not low enough to repair a percentage of erasures as high as $70 \%\left(R_{\text {early }} \geq 1\right)$. In a similar manner, UL configurations with more than $50 \%$ of information in the late part are not included in the table since they are generally not practical in real scenarios.

\subsubsection{Simulation Results}

The simulation results presented in this section are aimed at the performance evaluation of long TI in DVB-NGH from the point of view of time diversity and zapping time. The parameters used for the simulations are listed in Table 5.2. These are very similar to the simulation parameters used in Chapter 4 for the investigation of time and space diversity in DVB-T and DVB-T2 systems.

\section{Early Decoding}

Fig. 5.3 depicts the performance of early decoding after the reception of one frame for the configurations listed in Table 5.1. We have also represented the curves corresponding to late decoding with code rates $2 / 5,7 / 15(\approx 1 / 2)$, $2 / 3$, and $11 / 15$ for the sake of comparison. In the figure, we can see how the performance of early decoding deteriorates when a lower percentage of information is carried in the late part. This illustrates the trade-off that exists between zapping performance and time diversity in DVB-NGH: uniform interleaving provides better diversity over time but diminishes the capabilities of receivers to perform fast zapping. Lower code rates are capable to repair a larger percentage of erasures within the codewords, and therefore, they reduce the performance degradation in early decoding. For example, going from UL $50 \%$ to UL $40 \%$ requires an extra $8 \mathrm{~dB}$ to achieve fast zapping after the first information frame with code rate $1 / 3$, but only $2 \mathrm{~dB}$ with code rate $1 / 5$. 
CHAPTER 5. COMBINED DIVERSITY IN NEXT GENERATION SYSTEMS

Table 5.2: Simulation Parameters for DVB-NGH

\begin{tabular}{c|c}
\hline \multicolumn{1}{c||}{ System Parameters } & Value \\
\hline \hline Bandwidth & $8 \mathrm{MHz}$ \\
\hline FFT mode & $8 \mathrm{~K}$ \\
\hline Guard interval & $1 / 4$ \\
\hline Channel estimation & Ideal \\
\hline QAM demapping & Optimum soft maximum a posteriori (MAP) \\
\hline QoS criterion & BU FER $1 \%$ \\
\hline Channel models & NGH Outdoor \\
\hline Doppler $\left(f_{d}\right)$ & $33 \mathrm{~Hz}(60 \mathrm{~km} / \mathrm{h}$ at $600 \mathrm{MHz})$ \\
\hline Shadowing parameters & Standard deviation $(\sigma) 5.5 \mathrm{~dB}$ \\
& Correlation distance $\left(d_{c o r r}\right) 20 \mathrm{~m}$ \\
\hline Channel separation $\left(f_{s}\right)$ & $100 \mathrm{MHz}$ \\
\hline \hline
\end{tabular}

The results also show that there is a performance loss in early decoding compared to the equivalent code rate in late decoding. This is caused by the low robustness of LDPC codes against heavy puncturing in DVB-NGH. In particular, the performance loss of early decoding compared to the equivalent non-punctured code is larger with uniform interleaving and with higher code rates. For code rate 1/3, the loss with UL $50 \%$ and UL $45 \%$ is around $1 \mathrm{~dB}$ and $2 \mathrm{~dB}$ respectively compared to the equivalent code rates $2 / 3$ and $11 / 15$. For code rate $1 / 5$, the loss with UL $50 \%$, UL $40 \%$ and UL $30 \%$ is around $1 \mathrm{~dB}$, $2 \mathrm{~dB}$ and $3 \mathrm{~dB}$ respectively compared to the equivalent code rates $2 / 5,7 / 15$, and $2 / 3$.

\section{Late Decoding}

Fig. 5.4 illustrates the performance of late decoding with different TI configurations. In the left side of the figure, the results show the BBFER curves of intra-frame BI $\left(T_{\mathrm{TI}}=200 \mathrm{~ms}\right)$ and inter-frame $\mathrm{CI}\left(T_{\mathrm{TI}}=10 \mathrm{~s}\right)$ with uniform and UL configurations. The outage probability of the channel for $200 \mathrm{~ms}$ and $10 \mathrm{~s}$ of TI is also shown as reference. This has been computed according to the methodology described in Chapter 3, and corresponds to the highest performance that can be achieved by any communication system. In the figure, 

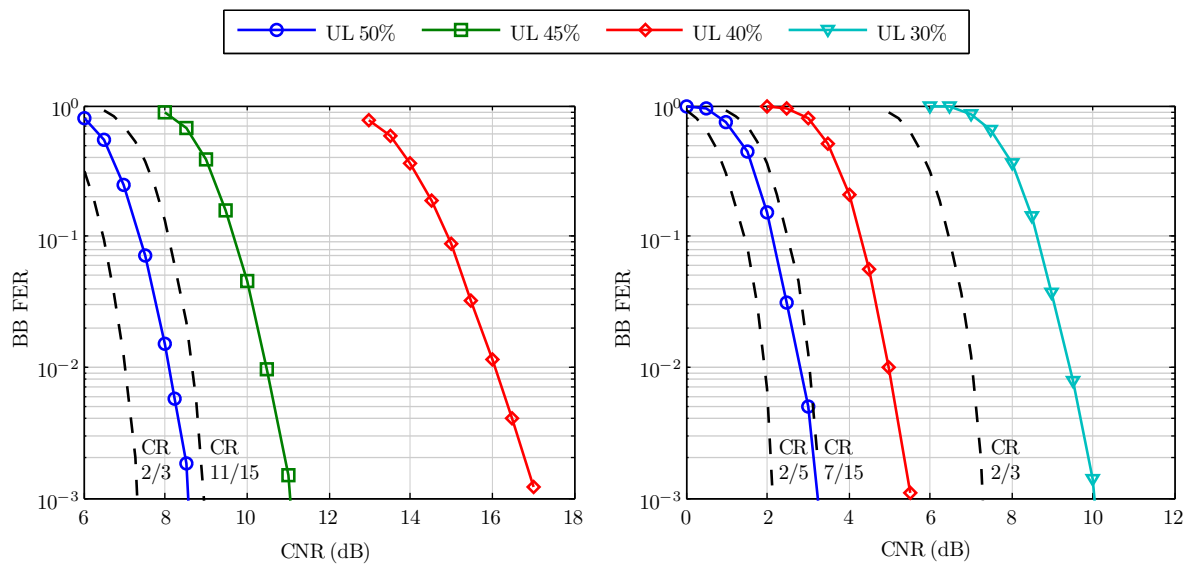

Figure 5.3: Performance of early decoding with different UL interleaving configurations for QPSK $1 / 3$ (left) and QPSK $1 / 5$ (right) in the TU6 channel $\left(f_{d}=33 \mathrm{~Hz}\right)$. Dashed lines correspond to late decoding for equivalent code rates.

we can see the trade-off between zapping performance and time diversity that exists in DVB-NGH: UL interleaving improves the fast zapping capabilities of receivers but reduces the reception robustness in late decoding as a result of worse time diversity. The greater the percentage of information carried in the late part, the higher the degradation. According to the results, the performance loss compared to uniform interleaving is around $0.5 \mathrm{~dB}, 1 \mathrm{~dB}$ and $2 \mathrm{~dB}$ for UL $30 \%$, UL $40 \%$ and UL $50 \%$ respectively. On the other hand, the performance gap between DVB-NGH and the outage capacity of the channel is larger with inter-frame CI than with intra-frame BI. This is explained by the influence of shadowing in the performance of BICM systems. With intra-frame $\mathrm{BI}$, the interleaving duration is much shorter than the coherent time of the channel, and thus, the presence of shadowing results in an offset on the average CNR of each codeword. With inter-frame CI, the interleaving duration covers several coherence times, and in this case, the system performance depends on the error-correction capabilities of the FEC code against shadowing.

In the right side of Fig. 5.4, the results show the gain of long TI compared to intra-frame BI for code rates between $1 / 3$ and $11 / 15$. The dashed line has been computed according to the outage capacity of the channel, and represents the highest gain that can be achieved with ideal FEC and interleaving. Due to the fact that it is based on Shannon limit, the gain of long TI in this case does not depend on particular aspects of BICM systems such as the constellation or the code rate. On the contrary, the gain of long TI in DVB-NGH diminishes with 


\section{-}
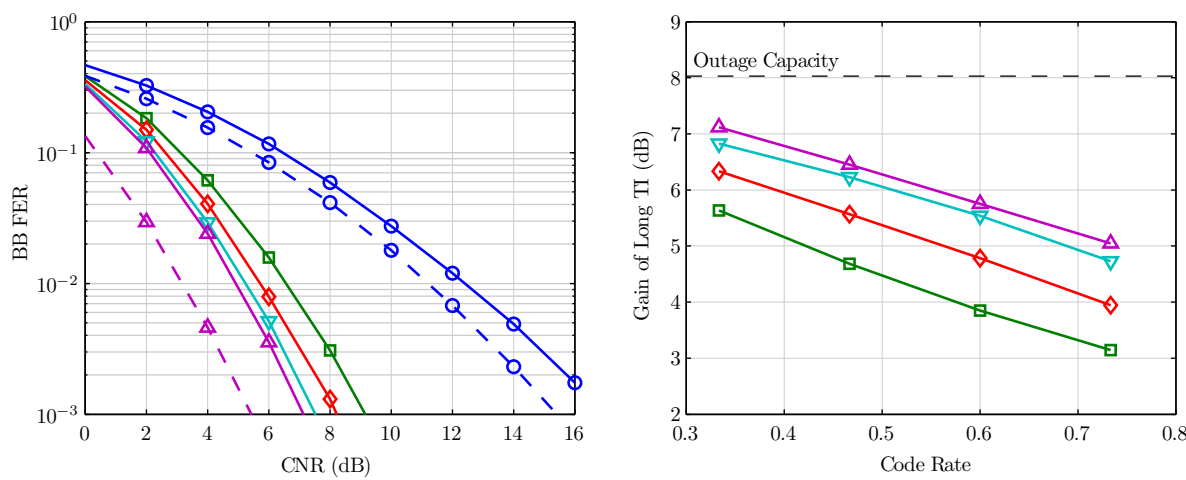

Figure 5.4: Performance of DVB-NGH with different configurations of TI in the TU6 channel with shadowing $\left(f_{d}=33 \mathrm{~Hz}, \sigma=5.5 \mathrm{~dB}\right.$ and $\left.d_{\text {corr }}=20 \mathrm{~m}\right)$. On the left, BBFER curves with QPSK 1/3. On the right, gain of long TI compared to intra-frame BI $\left(T_{\mathrm{TI}}=200 \mathrm{~ms}\right)$ with QPSK and different code rates. Dashed lines correspond to the outage probability of the channel.

higher code rates, since it is conditioned by the error-correcting capabilities of the FEC code. This means that in order to take full advantage of long TI and approach the outage capacity of the channel, it is necessary to employ low code rates as well as uniform interleaving.

\section{BB-iFEC}

Despite the fact that a single FEC solution with uniform interleaving achieves the best performance in both stationary and mobile channels, a split FEC approach (i.e. two concatenated FEC codes) can provide better memory efficiency, fast zapping performance, and compatibility at the expense of some performance loss.

BB-iFEC is a split FEC scheme aimed to provide long TI at the physical layer with lower zapping times. As shown in Fig. 5.5, it features an outer FEC and an outer time interleaver concatenated to the inner FEC and inner time interleaver. In practice, the inner and outer FEC are the same, e.g. LDPC code, so that only one hardware FEC chain is needed in receivers. The inner time interleaver operates on a frame level and provides an interleaving duration in the order of hundreds of milliseconds, whereas the outer time interleaver extends the protection across multiple frames and up to several seconds. In the figure, we can see that the outer time interleaver performs the iFEC data spreading 


\subsection{Time Diversity}

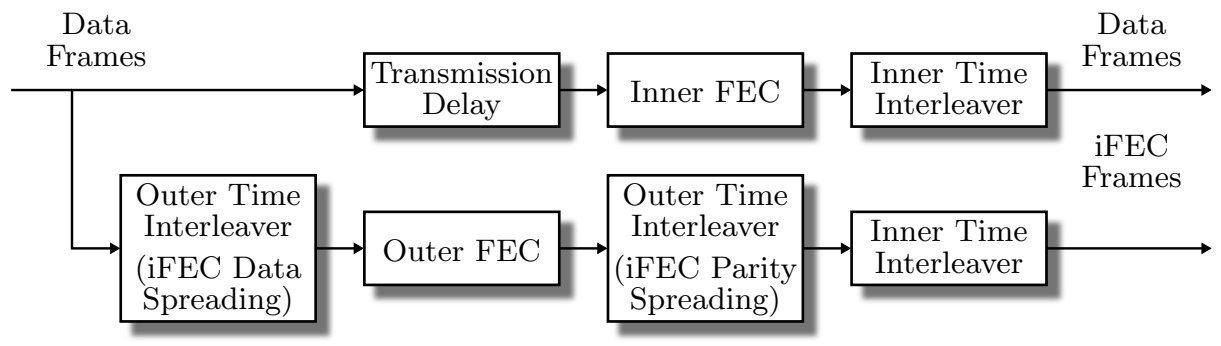

Figure 5.5: BB-iFEC transmission chain.

and the iFEC parity spreading before and after the outer FEC respectively. These are based on MPE-iFEC and operate under the same parameters: data spreading factor $B$, parity spreading factor $S$, and data delay $D$. An in-depth explanation of BB-iFEC can be found in [111].

In Fig. 5.6 we compare the single FEC approach adopted in DVB-NGH with BB-iFEC and AL-FEC in stationary and mobile reception (AWGN and TU6 channel respectively). Single FEC has been configured with code rate $1 / 3$, $\mathrm{BB}-\mathrm{iFEC}$ has been configured with code rate $2 / 3$ at the inner $\mathrm{FEC}$ and code rate $2 / 5$ at the outer FEC, and AL-FEC has been configured with code rate $2 / 3$ at the physical layer and $1 / 2$ at the application layer. In all three cases, the overall code rate is approximately $1 / 3$. In stationary reception, the best performance is achieved by single FEC, followed by BB-iFEC and AL-FEC. BB-iFEC outperforms AL-FEC in approximately $3 \mathrm{~dB}$ and loses only around $1 \mathrm{~dB}$ compared to single FEC despite having two different FEC stages. It is important to note that TI does not provide any benefit in stationary channels and thus, the performance of uniform and UL interleaving with single FEC is the same. Also, the performance of AL-FEC is given by the code rate at the physical layer $(2 / 3)$, as the additional protection given at upper layers does not provide any gain when the user is not moving across the service area. In mobile reception, BB-iFEC achieves the same performance as single FEC with a UL $50 \%$ configuration, losing around $2 \mathrm{~dB}$ compared to uniform interleaving. The good performance of BB-iFEC in mobile scenarios is explained by the fact that, while it employs a sub-optimal approach based on the concatenation of two different FEC stages, the information is interleaved in a uniform manner. On the other hand, splitting the protection between the physical and upper layers with AL-FEC provides the worst performance, losing around $4 \mathrm{~dB}$ compared to BB-iFEC, and around $6 \mathrm{~dB}$ compared to single FEC with uniform interleaving. Altogether, the results reveal that moving the time diversity into the physical layer with BB-iFEC is beneficial for reception robustness compared to UL-FEC 

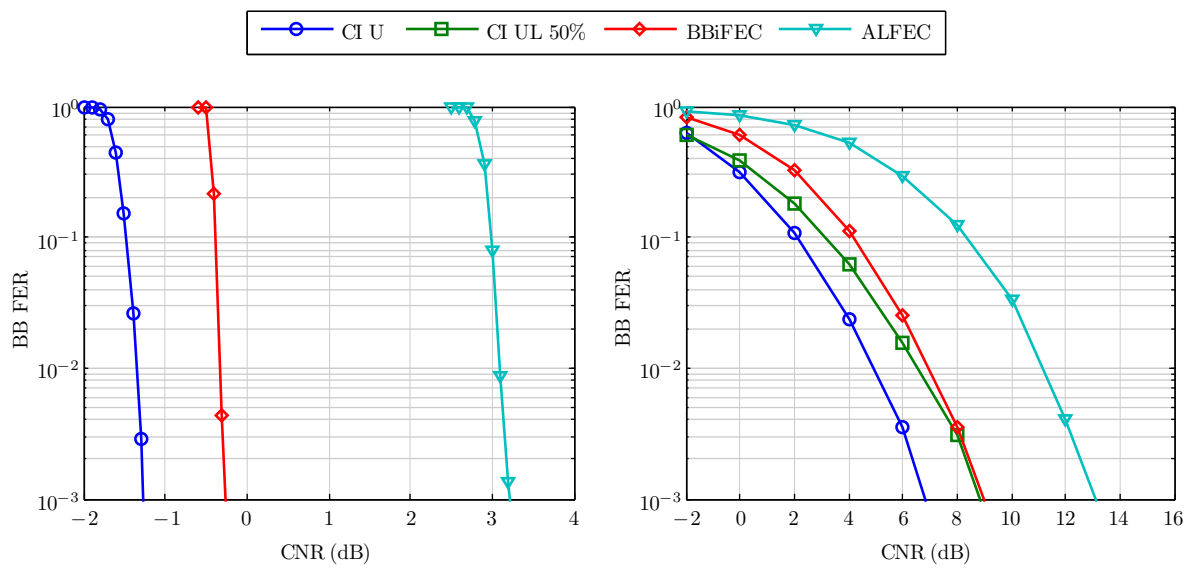

Figure 5.6: Comparison between single FEC, BB-iFEC, and AL-FEC in DVB-NGH (QPSK 1/3). On the left, stationary reception in AWGN. On the right, mobile reception in the TU6 channel with shadowing $\left(f_{d}=33 \mathrm{~Hz}, \sigma=5.5 \mathrm{~dB}\right.$ and $\left.d_{\text {corr }}=20 \mathrm{~m}\right)$.

protection, while the split FEC approach presents an advantage in terms of zapping time compared to the single FEC solution adopted in DVB-NGH.

\subsection{Frequency Diversity}

The incorporation of TFS in DVB-NGH offers potentially better frequency diversity by extending the interleaving across hundreds of $\mathrm{MHz}$ in the UHF band. When TFS is used, the services are multiplexed across several RF channels by means of frequency hopping and time slicing (see Section 2.5.2). This allows extending the interleaving in the frequency domain beyond one RF channel and across hundreds of MHz. DVB-NGH incorporates two different modes of operation with TFS. In the first mode of operation, referred to as intra-frame TFS, frequency hopping is performed within the NGH frames and between different sub-slices. The second mode of operation combines TFS with inter-frame interleaving so that frequency hopping is performed between different NGH frames. In this section, we focus on the gains of intra-frame TFS, whereas the combination of TFS with inter-frame interleaving is investigated in Section 5.4.

Fig. 5.7 illustrates the performance of TFS in DVB-NGH according to the number of RF channels that are grouped with TFS. The separation between RF channels, $f_{s}$, has been selected to $100 \mathrm{MHz}$. In this manner, the RF channels employed in the simulations are those located in $470 \mathrm{MHz}, 570 \mathrm{MHz}, 670 \mathrm{MHz}$ 


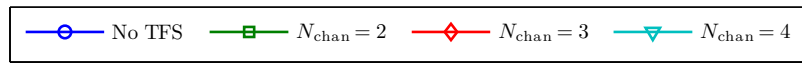
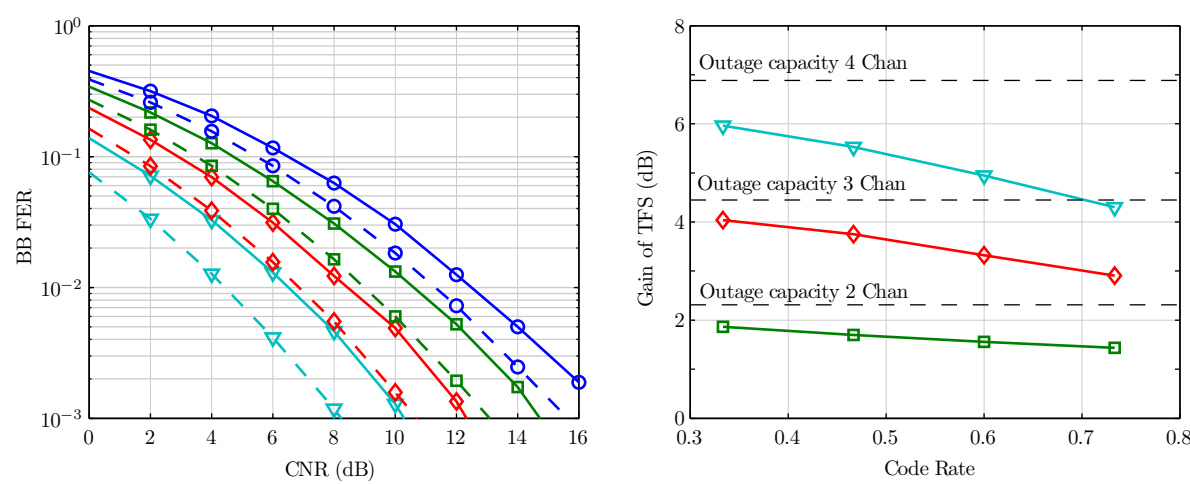

Figure 5.7: Performance of TFS in the TU6 channel with shadowing $\left(f_{d}=33 \mathrm{~Hz}, d_{\text {corr }}=\right.$ $20 \mathrm{~m}, \sigma=5.5 \mathrm{~dB}$ and $f_{s}=100 \mathrm{MHz}$ ). On the left, BBFER curves with QPSK $1 / 3$. On the right, gain of TFS with QPSK and different code rates. Dashed lines correspond to the outage probability of the channel.

and $770 \mathrm{MHz}$, which constitutes a realistic configuration in the context of European broadcasting. The results in the left side of the figure show the BBFER curves with QPSK 1/3. The dashed lines represent the outage probability of the channel for the same interleaving duration $\left(T_{\mathrm{TI}}=200 \mathrm{~ms}\right)$ and Doppler $(33 \mathrm{~Hz})$ than the physical layer simulations. In this case, the achievable gain in real DVB-NGH systems is consistent with the information-theoretic results presented in Chapter 3, and gains of around $6 \mathrm{~dB}$ are feasible when the interleaving extends across four RF channels $(300 \mathrm{MHz})$. From the results, we can also see that the performance gap between DVB-NGH and the outage channel capacity increases with the number of RF channels. This is similar to the case of long TI in the sense that, although the utilization of extended interleaving improves the reception robustness as a result of better diversity, the gain depends on the error-correcting capabilities of the FEC code (i.e. code rate).

In the right side of Fig. 5.7, we can see more clearly the influence of the code rate in the performance gains that can be achieved with TFS. The dashed lines have been computed according to the outage capacity of the channel, and represent the highest gain that can be achieved with ideal FEC and interleaving over two, three and four RF channels with $f_{s}=100 \mathrm{MHz}$. The results show two important aspects regarding the performance of TFS in BICM systems, and particularly, DVB-NGH. On one hand, the distance to the outage capacity of the channel increases when more RF channels are grouped in the same TFS 


\section{CHAPTER 5. COMBINED DIVERSITY IN NEXT GENERATION SYSTEMS}

multiplex, as in this case, it is necessary to cope with a larger signal variability in the frequency domain. On the other hand, the gain of TFS diminishes when lower code rates are used. As can be seen in Fig. 5.7, the higher the number of channels, the greater the dependency of the system performance on the code rate.

\subsection{Space Diversity}

DVB-NGH distinguishes between rate 1 and rate 2 MIMO depending on the number of information symbols that are transmitted per subcarrier. Rate 1 MIMO schemes provide better diversity but transmit only one information symbol per subcarrier. Rate 2 MIMO schemes also exploit the multiplexing capabilities of the channel by transmitting two information symbols per subcarrier (see Chapter 2). The techniques adopted in the standard for rate 1 MIMO are eSFN and the Alamouti code, and can be used for spectral efficiencies ranging from $2 \mathrm{bpc}$ (i.e. QPSK) up to $8 \mathrm{bpc}$ (i.e. 256QAM). In the case of rate $2 \mathrm{MIMO}$, the standard has adopted eSM for spectral efficiencies of $6 \mathrm{bpc}, 8 \mathrm{bpc}$, and $10 \mathrm{bpc}$. The first configuration is achieved by transmitting QPSK symbols on one antenna and 16QAM symbols on the other, the second configuration is achieved by transmitting 16QAM symbols on both antennas, and the third configuration is achieved by transmitting 16QAM symbols on one antenna and 64QAM symbols on the other.

The BBFER curves corresponding to SISO, SIMO, MIMO eSFN, MIMO Alamouti, and MIMO eSM are shown in Fig. 5.8. The dashed lines represent the outage probability of the channel with the same interleaving duration and Doppler. For the simulations, we have considered the NGH outdoor channel model with $33 \mathrm{~Hz}$ of Doppler and $200 \mathrm{~ms}$ of TI. Due to the fact that the performance of MIMO depends on the CNR, the curves have been computed for two different configurations of MODCOD (2 bpc 1/3 on the left, and 8 bpc 11/15 on the right) covering a wide range of CNR values. We can see that MIMO eSM achieves the best performance followed by MIMO Alamouti, MIMO eSFN, SIMO and SISO. It is important to notice that although MIMO eSFN is outperformed by MIMO Alamouti, the performance gap between both schemes is at most $0.5 \mathrm{~dB}$. On the other hand, the performance gap between DVB-NGH and the outage capacity of the channel is significantly larger with MIMO than with SIMO or SISO. The reason is that the implementation of iterative decoding and demapping techniques is necessary to fully exploit the diversity and multiplexing capabilities of the MIMO channel [112].

In Fig. 5.9, we show the capacity curves of SISO, SIMO, MIMO eSFN, MIMO Alamouti, and MIMO eSM for $2 \mathrm{bpc}$ and $8 \mathrm{bpc}$. These curves repre- 

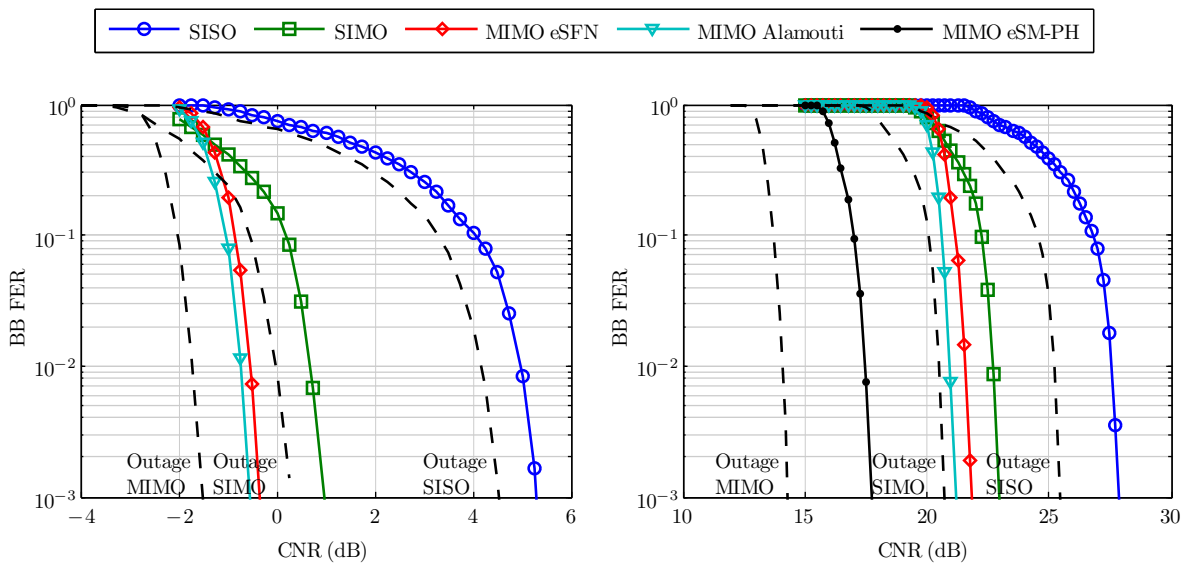

Figure 5.8: BBFER curves of DVB-NGH in the NGH outdoor mobile channel $\left(f_{d}=33 \mathrm{~Hz}\right)$. On the left, rate 1 MIMO schemes with 2 bpc $1 / 3$. On the right, rate 1 and rate 2 MIMO schemes with 8 bpc 11/15. Dashed lines represent the outage probability of the channel.

sent the spectral efficiency that can be achieved for any given value of CNR at BBFER $1 \%$. The lowest point in the curves corresponds to code rate $1 / 3$ and the highest point to code rate $11 / 15$. The results in the right side of the figure take into account the additional overhead introduced by pilot information, whereas the results in the left side do not. In particular, we have assumed the same considerations as in the NGH standardization process, i.e. pilot overhead 1/12 for SISO, SIMO, and eSFN, and pilot overhead 1/6 for the Alamouti code and eSM. This corresponds to a robust configuration that provides sufficient quality of channel estimation in mobile scenarios.

If the pilot overhead is not taken into account, the advantage of MIMO Alamouti over MIMO eSFN is negligible for low code rates, although it increases with higher code rates up to approximately $0.5 \mathrm{~dB}$. If we consider the pilot overhead, eSFN outperforms MIMO Alamouti with every code rate, as the better reception robustness achieved by the Alamouti code in terms of CNR does not compensate for the higher pilot overhead in terms of spectral efficiency. On the other hand, MIMO eSM outperforms the two rate 1 MIMO schemes in the case of $8 \mathrm{bpc}$ as a result of better multiplexing capabilities. Although the gain of MIMO eSM over MIMO eSFN diminishes when the pilot overhead is taken into account, MIMO eSM still provides the best performance with any code rate. Furthermore, the advantage of MIMO eSM grows larger with higher code rates due to the relationship between the multiplexing gain and the CNR as explained in Chapter 3. 

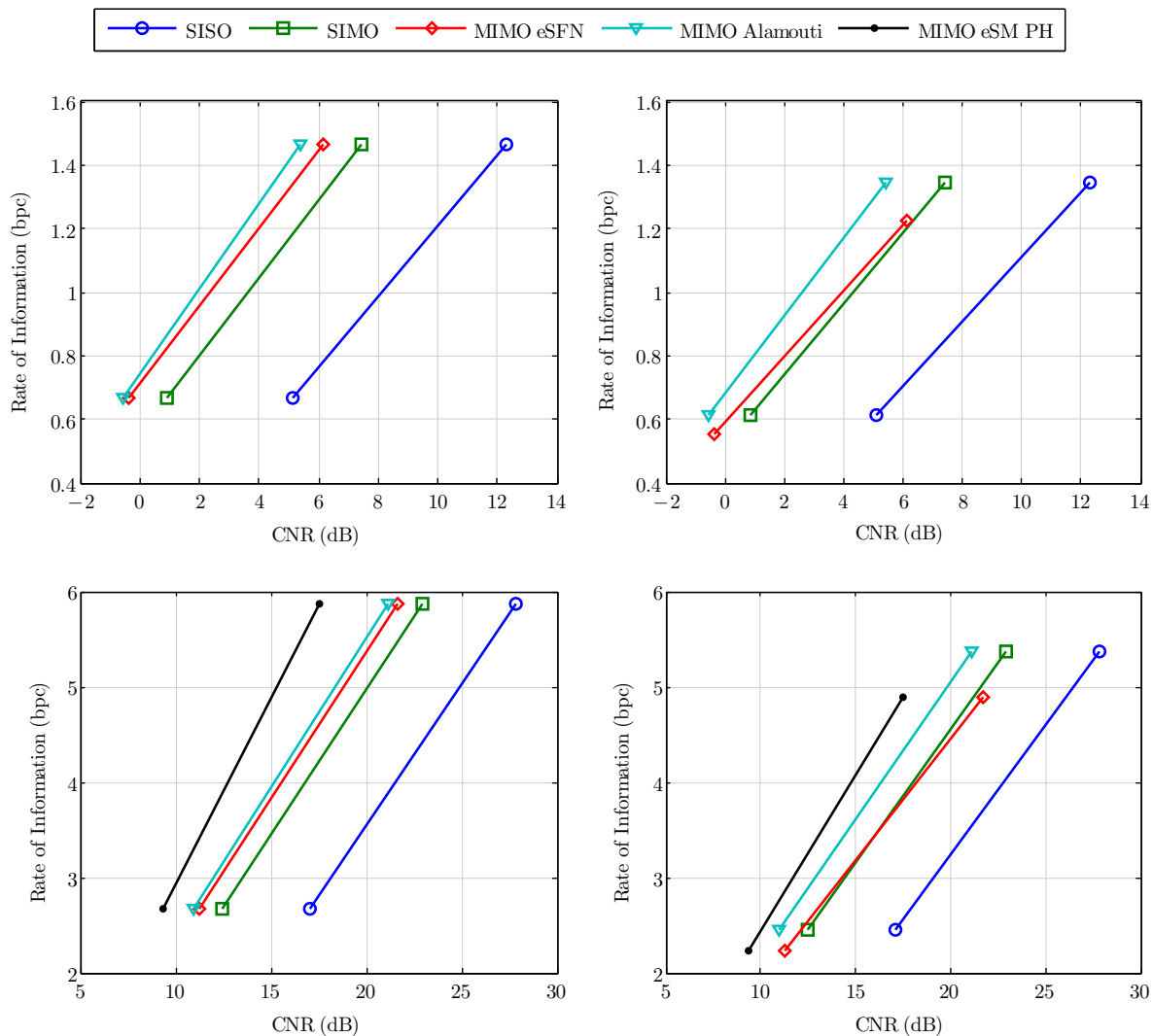

Figure 5.9: Capacity curves of DVB-NGH with the MIMO techniques adopted in the standard in the NGH outdoor mobile channel $\left(f_{d}=33 \mathrm{~Hz}\right)$. On the right, the curves include the pilot overhead, whereas the curves on the left side do not. The curves correspond to 2 bpc (top) and $8 \mathrm{bpc}$ (bottom) with code rates $1 / 3$ and $11 / 15$.

It is important to note that the performance of some MIMO techniques depends on the error-correcting capabilities of the FEC code (i.e. code rate). MIMO techniques such as eSFN and eSM rely on the FEC code to exploit the diversity between the transmit antennas, similarly to how time and frequency diversity is achieved by means of FEC and interleaving. Other techniques like the Alamouti code and the Golden code introduce the diversity into the information symbols at the expense of higher demodulation complexity.

To better illustrate the influence of the FEC code on the performance of MIMO techniques, in Fig. 5.10 we compare the BBFER curves of MIMO eSFN 

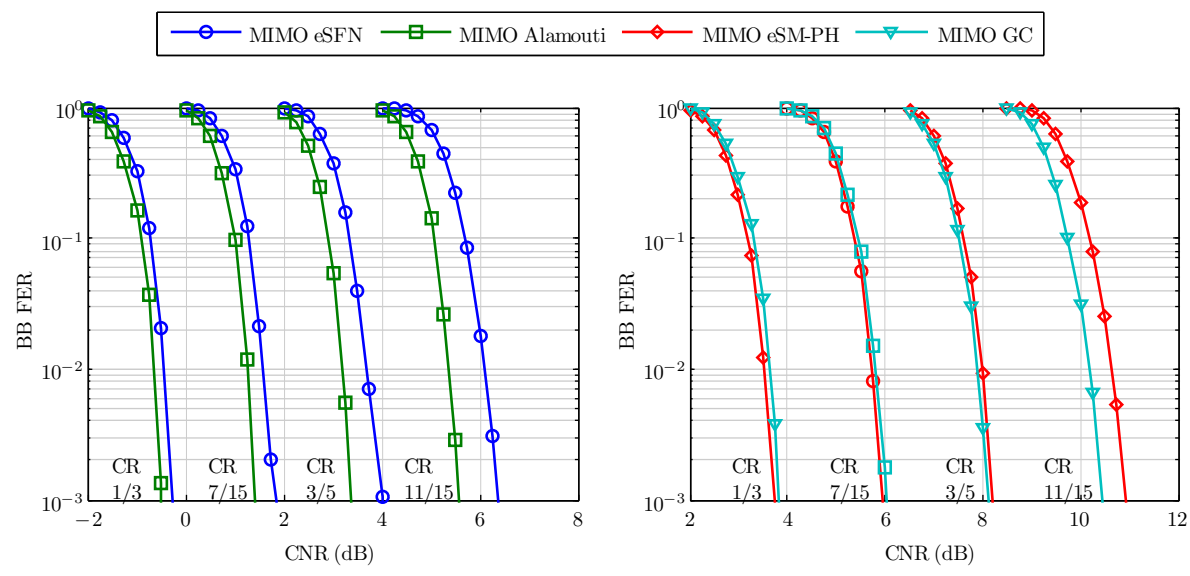

Figure 5.10: Comparison between MIMO schemes according to the code rate in the NGH outdoor mobile channel $\left(f_{d}=33 \mathrm{~Hz}\right)$. On the left, comparison between MIMO eSFN and MIMO Alamouti with 2 bpc $1 / 3$. On the right, comparison between MIMO eSM and MIMO GC with 4 bpc $1 / 3$.

and MIMO Alamouti with different code rates on one hand, and the BBFER curves of MIMO eSM and MIMO GC on the other. Despite the fact that the Golden code is not included in DVB-NGH, here we use it for comparison purposes as an optimal rate 2 MIMO scheme that achieves the DMT frontier (i.e. full diversity and multiplexing gain). In the case of MIMO eSFN vs. MIMO Alamouti, we can see that although the latter achieves the best performance for any code rate, the advantage diminishes with lower code rates down to less than $0.2 \mathrm{~dB}$. In a similar manner, while MIMO GC outperforms MIMO eSM at high code rates, the gain decreases as the code rate goes down, and for code rates lower than $7 / 15$, MIMO eSM achieves the best performance. This resembles the use of rotated constellation in that the improvement of embedding the diversity into the information symbols increases with higher code rates, achieving very little gain when the rate of the FEC code is low.

\subsection{Combined Diversity}

In the previous sections of this chapter, we have evaluated in a separate manner the performance gains of time, frequency and space diversity in DVB-NGH. In this section, we focus on the combination of different types of diversity, and particularly, on the performance gains that can be achieved by combining long TI with TFS and MIMO. The results in the left side of Fig. 5.11 correspond 


\section{CHAPTER 5. COMBINED DIVERSITY IN NEXT GENERATION SYSTEMS}
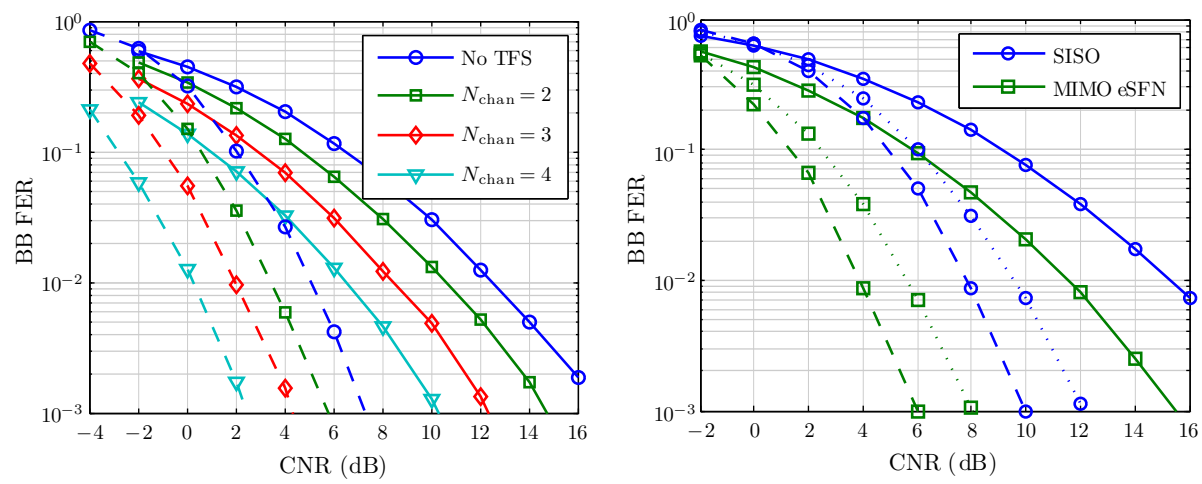

Figure 5.11: Combined diversity in DVB-NGH systems (QPSK 1/3). On the left, long TI with TFS in the TU6 channel with shadowing $\left(f_{d}=33 \mathrm{~Hz}, d_{\mathrm{corr}}=20 \mathrm{~m}, \sigma=5.5 \mathrm{~dB}\right.$ and $f_{s}=100 \mathrm{MHz}$ ). On the right, long TI with MIMO in the NGH outdoor channel with shadowing. Continuous lines correspond to intra-frame BI with $T_{\mathrm{TI}}=200 \mathrm{~ms}$, dashed lines correspond to uniform inter-frame CI with $T_{\mathrm{TI}}=10 \mathrm{~s}$ and dotted lines correspond to UL $50 \%$ inter-frame CI with $T_{\mathrm{TI}}=10 \mathrm{~s}$.

to the combination of long TI with TFS. We can see that the total gain of combining both techniques is lower that the sum of the individual gains due to the diminishing marginal returns of diversity. In this case, the gain of long TI with $T_{\mathrm{TI}}=10 \mathrm{~s}$ is $1 \mathrm{~dB}$ lower when the frequency interleaving extends across four $\mathrm{RF}$ channels $(300 \mathrm{MHz})$ instead of one $\mathrm{RF}$ channel $(8 \mathrm{MHz})$. Similarly, the gain of TFS with four RF channels is $1 \mathrm{~dB}$ lower when the TI extends over $10 \mathrm{~s}$ with inter-frame CI rather than $200 \mathrm{~ms}$ with intra-frame BI.

In the right side of Fig. 5.11, we show the results corresponding to the combination of long TI with MIMO eSFN. According to the figure, the total gain of combining both techniques is approximately equal to the sum of the individual gains. It is important to note that, in the case of cross-polarized antennas, the majority of the gain does not come from fading, but from better protection against rotation and asymmetry effects. These are considered to persist over periods of time much longer than the interleaving duration, and therefore, cannot be compensated by means of TI.

\subsection{Rotated Constellations}

Rotated constellations increase the diversity order of BICM systems at the expense of higher demodulation complexity (see Section 2.3). DVB-NGH inherits the 2DRC from DVB-T2, and also incorporates 4DRC for certain configura- 
Erasure event spanning four frames with 2DRC

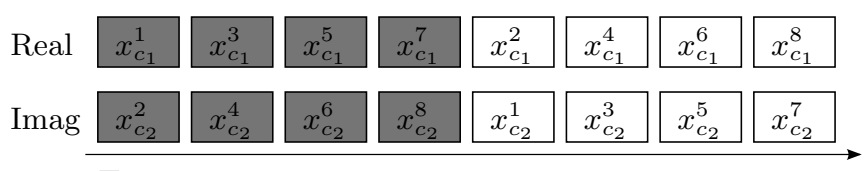

Time

Erasure event spanning six frames with $4 \mathrm{DRC}$

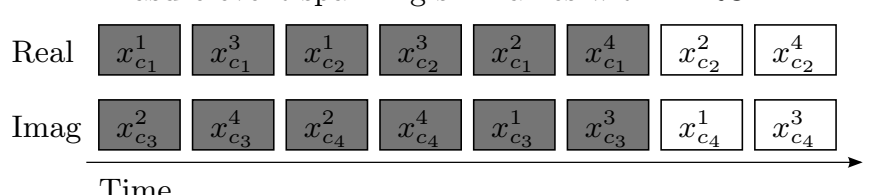

Time

Figure 5.12: Interleaving of components with inter-frame interleaving across eight frames.

tions (QPSK and code rates higher than 1/2). The improved protection of rotated constellations against fading makes them very useful to increase the performance gains of long TI and TFS. In the time domain, the signal outages encountered along the user trajectory might result in the loss of entire information frames and a very high number of errors within the codewords. In the case of UL interleaving with a $50 \%$ of information in the late part, losing a single frame can result in a percentage of errors as high as $50 \%$ for some codewords. In the frequency domain, the presence of interference from neighbouring networks added to frequency dependency of the transmitter site, the receiver and the propagation channel, might result in the loss of entire RF channels that cannot be recovered with regular, non-rotated constellations.

\subsubsection{Analysis of Component Interleaving in DVB-NGH}

In DVB-T2, the Q component of the rotated symbols is first cyclically delayed by one cell inside the FEC codewords in order to separate the two components of each symbol. Then, a cascade of cell, time, and frequency interleavers separates the components in the time and frequency domains in order to reduce the fading correlation. In DVB-NGH, the cyclic Q delay has been replaced by a more sophisticated component interleaver optimized for inter-frame interleaving and TFS. The component interleaver is designed in such a manner that, with interframe interleaving, the different components of each rotated symbol end up being transmitted in different frames, with the maximum possible separation between components inside the interleaving duration. If TFS is also enabled, the component interleaver assigns each component to a different RF channel in a cyclically manner. 


\section{CHAPTER 5. COMBINED DIVERSITY IN NEXT GENERATION SYSTEMS}

The main advantage of rotated constellations when they are used in combination with inter-frame interleaving and TFS, is that they can reduce the loss of information against signal outages in the time and frequency domain. We will use figures 5.12 and 5.13 as an example to illustrate the improvement that can be achieved in DVB-NGH by using rotated constellations. In Fig. 5.12, we show the distribution of components when the TI extends across eight frames. In the figure, $x_{C i}^{j}$ denotes the component $i$ of the rotated symbol $j$. With 2DRC, a signal outage extending across four or less consecutive frames in the time domain results in only one of the two components, I or Q, being erased for any rotated symbol. Therefore, it is possible to retrieve all the information at the receiver side when demodulating the signal by using one of the two components that has not been erased by the channel. On the contrary, the loss of five or more consecutive frames causes the erasure of both components for some rotated symbols. With $4 \mathrm{DRC}$, it is possible to suffer an outage extending up to six consecutive frames and still receive at least one component of each rotated symbols. Generally speaking, the maximum number of frames, $E_{\text {frame }}$, that can be lost while receiving at least one component of each rotated symbols is:

$$
E_{\text {frame }}=N_{\text {int }}\left(1-\frac{1}{D}\right)
$$

where $N_{\text {int }}$ denotes the number of frames covered by the interleaving duration and $D$ denotes the dimension of the rotation.

The previous analysis can be extended to the case when fast zapping is used. Let us assume UL interleaving with the $50 \%$ of information being transmitted in the late frame. In this case, the symbols corresponding to the first four frames in the previous example will be carried in a single frame that can be used by the receiver to start decoding the service and perform fast zapping. With rotated constellations, the receiver has at least one component of each information symbol after receiving the late frame, improving the probabilities to decode the service. The use of rotated constellations might also reduce the performance degradation of UL interleaving after zapping by reducing the loss of information when one or several frames are in deep fading.

In Fig. 5.13, we show the distribution of components when the frames are transmitted across four different RF channels by means of TFS. Here, we focus on the loss of one or multiple RF channels caused, for example, by the presence of interference from neighbouring networks. The distribution performed by the component and time interleavers places the components of each rotated symbol in a different RF channel. In this case, the maximum number of RF channels, $E_{\text {chan }}$, that can be lost while receiving at least one component of each rotated symbol is equal to the dimension of the rotation, $D$. 
Erasure event spanning one $\mathrm{RF}$ channel with $2 \mathrm{DRC}$

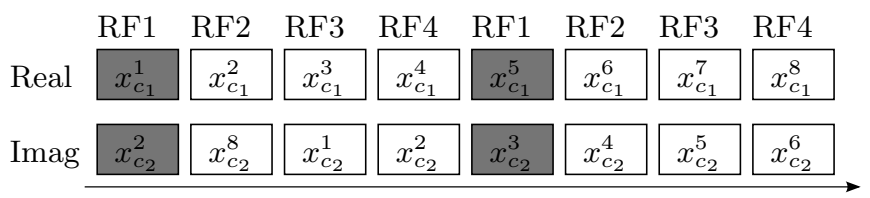

Time

Erasure event spanning three RF channel with 4DRC

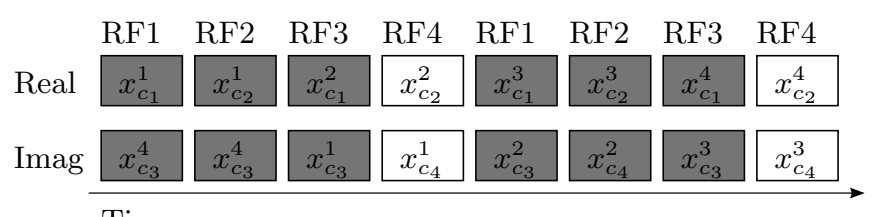

Time

Figure 5.13: Interleaving of components with inter-frame interleaving and TFS across eight frames and four RF channels.

\subsubsection{Simulation Results}

\section{Time Diversity with Long TI}

We first investigate the utilization of rotated constellations to improve the reception robustness (time diversity) with long TI. In the left side of Fig. 5.14, we show the BBFER curves corresponding to three different configurations of TI in DVB-NGH (QPSK 1/3). As can be seen in the figure, the gain of rotated constellations not only depends on the code rate, but also on the interleaving duration and the type of interleaving (i.e. uniform or UL). In this case, rotated constellations do not improve the performance for intra-frame interleaving $\left(T_{\mathrm{TI}}=200 \mathrm{~ms}\right)$, while there is a gain of around $0.1 \mathrm{~dB}$ and $0.5 \mathrm{~dB}$ for interframe interleaving $\left(T_{\mathrm{TI}}=10 \mathrm{~s}\right)$ with the uniform and UL $50 \%$ configurations respectively.

In the right side of Fig. 5.14, we represent the gain of 2DRC and 4DRC for different code rates and configurations of TI. It should be pointed out that, although we show the values corresponding to $4 \mathrm{DRC}$ with code rates lower than $1 / 2$ for the sake of comparison, they are not supported in the DVB-NGH standard. According to the results presented in Fig. 5.14, the gains of rotated constellations with intra-frame interleaving are very similar to those presented in Section. 3.3, which were obtained from an information-theoretic point of view in the i.i.d. Rayleigh channel. This is explained by the fact that, with interleaving durations in the order of hundreds of milliseconds, rotated constellations can only compensate for the presence of fast fading in the received signal. In 

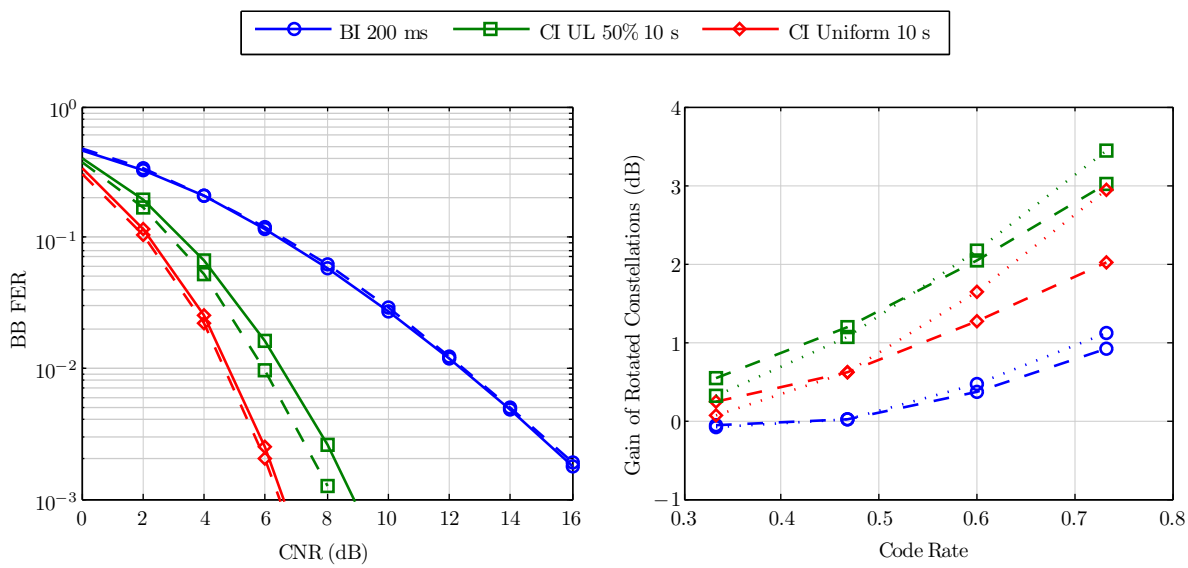

Figure 5.14: Performance of rotated constellations in DVB-NGH with different configurations of TI in the TU6 channel with shadowing $\left(f_{d}=33 \mathrm{~Hz}, \sigma=5.5 \mathrm{~dB}\right.$ and $\left.d_{\mathrm{corr}}=20 \mathrm{~m}\right)$. On the left, BBFER curves with QPSK 1/3. On the right, gain of rotated constellations with QPSK. Dashed lines correspond to 2DRC and dotted lines to 4DRC.

contrast, the gain of rotated constellations is significantly larger with interframe interleaving, as in this case, the interleaving duration is sufficiently long $(10 \mathrm{~s})$ to compensate for shadowing. We can also see that 4DRC constellations outperform $2 \mathrm{DRC}$ with code rates higher than $1 / 2$ in the three configurations of TI. It is interesting to note that the advantage of $4 \mathrm{DRC}$ over $2 \mathrm{DRC}$ is largest with uniform interleaving, since this achieves a better distribution of components from a time diversity point of view.

\section{Frequency Diversity with TFS}

We now focus on the gains of rotated constellations when the inter-frame interleaving extends across multiple RF channels by means of TFS. In the left side of Fig. 5.15, the results show the BBFER curves corresponding to one, two and four RF channels with $f_{s}=100 \mathrm{MHz}$. Therefore, the extent of interleaving in the frequency domain is $8 \mathrm{MHz}, 100 \mathrm{MHz}$ and $300 \mathrm{MHz}$ respectively. Similarly to the case with long TI, the gain of rotated constellations with TFS does not depend only on the code rate, but also on the extent of interleaving in the frequency domain. In this case (QPSK 7/15), the gain varies between $0 \mathrm{~dB}$ with one RF channel up to almost $1 \mathrm{~dB}$ with four RF channels.

In the right side of Fig. 5.15, the results show the gain of 2DRC and 4DRC for code rates between $1 / 3$ and $11 / 15$. We can see how the gain grows with higher code rates and when more RF channels are used in TFS. It should 

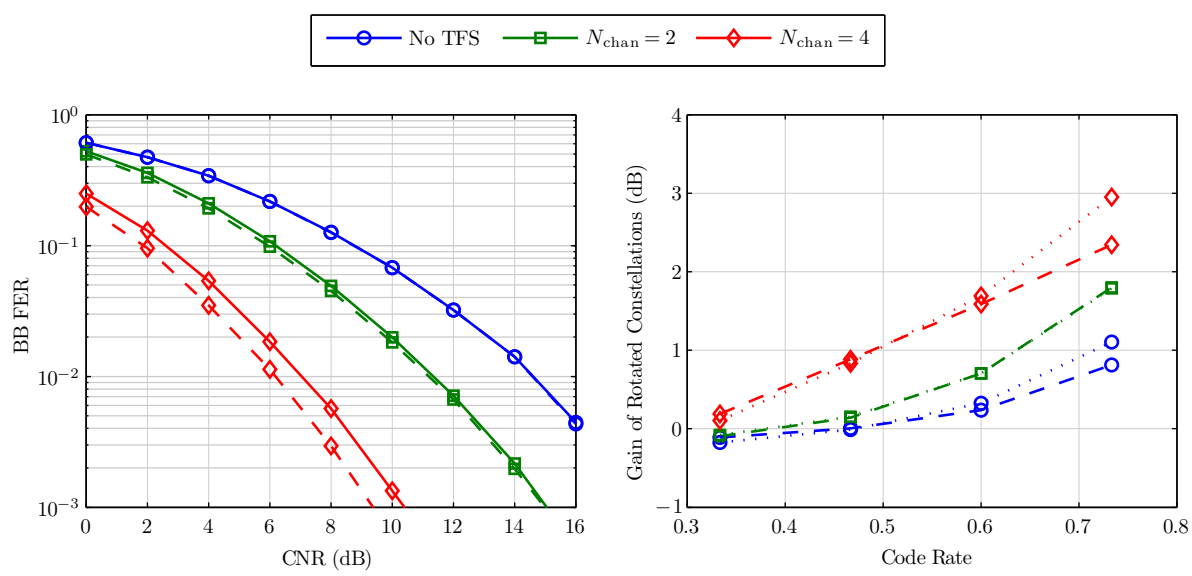

Figure 5.15: Performance of rotated constellations in DVB-NGH with different configurations of TFS in the TU6 channel with shadowing $\left(f_{d}=33 \mathrm{~Hz}, \sigma=5.5 \mathrm{~dB}, d_{\text {corr }}=20 \mathrm{~m}\right.$ and $f_{s}=100 \mathrm{MHz}$ ). On the left, BBFER curves with QPSK 7/15. On the right, gain of rotated constellations with QPSK. Dashed lines correspond to 2DRC and dotted lines to 4DRC.

be pointed out that, as with long TI, the utilization of rotated constellations with TFS improves the performance of DVB-NGH for code rates as low as $1 / 3$. It is also interesting to note that while 4 DRC generally outperform $2 \mathrm{DRC}$, they provide the same performance with two RF channels. Therefore, we can determine that in order to take advantage of the higher dimensionality of 4DRC, it is necessary to extend the component interleaving across more than two RF channels.

\section{Zapping Performance with Long TI}

In addition to increase the diversity gains of long TI and TFS in the time and frequency domains, rotated constellations can be used to lower the zapping time in DVB-NGH systems.. In the left side of Fig. 5.16, we focus on the reception robustness after receiving the first information frame (zapping time lower than $1 \mathrm{~s}$ ) when the TI duration is $10 \mathrm{~s}$. The results show the BBFER curves corresponding to UL interleaving with a $50 \%, 40 \%$ and $30 \%$ of information in the late frame. We can see that 2DRC (represented in dashed lines) improve the robustness of fast zapping with UL 50\% and UL 40\% (50\% and $60 \%$ of erasures) in more than $1 \mathrm{~dB}$. Moreover, they enable the reception with UL $30 \%$ and UL $20 \%$ ( $70 \%$ and $80 \%$ of erasures), which is not possible with non-rotated constellations. This is consistent with the results based on mutual 

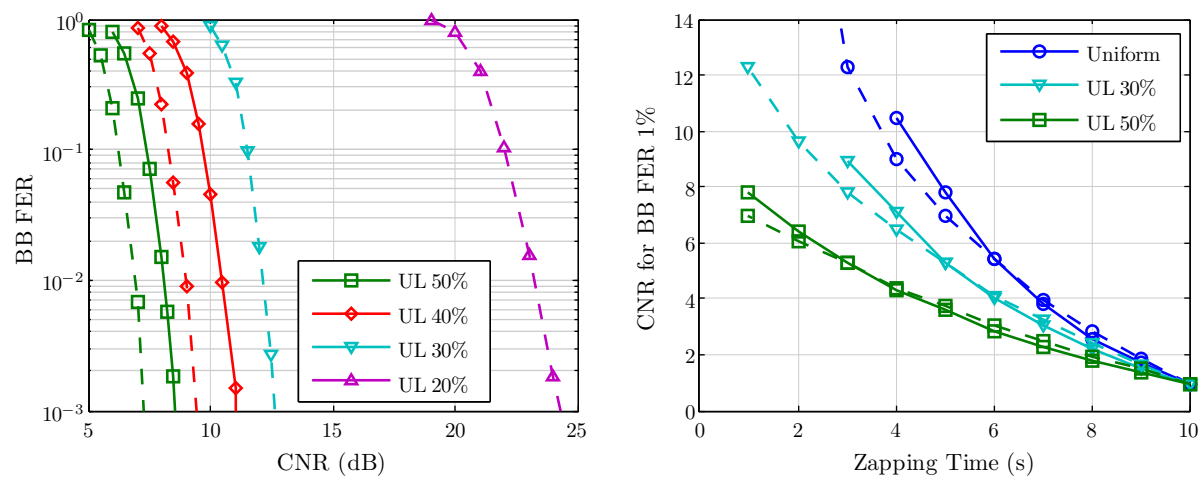

Figure 5.16: Zapping performance of DVBNGH (QPSK 1/3) with different configurations of TI $\left(T_{\mathrm{TI}}=10 \mathrm{~s}\right)$ in the TU6 channel $\left(f_{d}=33 \mathrm{~Hz}\right)$. On the left, BBFER curves with fast zapping after the first information frame. On the right, reception robustness according to the zapping time. Dashed lines correspond to 2DRC.

information presented in Fig. 3.3, where it is shown that the combination of code rate $1 / 3$ and $2 \mathrm{DRC}$ can repair a percentage of erasures up to $80 \%$.

In the right side of Fig. 5.16, we compare the performance of non-rotated constellations and 2DRC according to the zapping time when the interleaving duration is $10 \mathrm{~s}$. The longer the zapping time, the lower the CNR that is required to decode the service, since a larger percentage of information is available at the receiving side. After $10 \mathrm{~s}$ of zapping time, the receiver has all the information for start decoding the service, and thus, the highest protection is achieved. According to the results, the advantage of rotated constellations is higher with uniform interleaving than with UL interleaving. In this case, 2DRC improve the reception robustness of fast zapping for zapping times lower than $3 \mathrm{~s}$ with UL $50 \%$, lower than $5 \mathrm{~s}$ with UL $30 \%$, and lower than $6 \mathrm{~s}$ with uniform interleaving. We can also see that the utilization of 2DRC lowers in about $2 \mathrm{~s}$ the minimum zapping time that can be achieved with uniform and UL 30\% interleaving.

\subsection{Conclusions}

In this Chapter, we have investigated the use of time, frequency and space diversity in DVB-NGH systems by means of physical layer simulations. We have first evaluated each type of diversity in a separate manner. In the case of time diversity, the simulation results show that in order to take full advantage 
of long TI and approach the outage capacity of the channel, it is necessary to employ low code rates as well as uniform interleaving. In this sense, the utilization of UL interleaving can reduce the zapping time at the expense of worse time diversity. In addition to the single FEC solution adopted in the standard, we have also considered the utilization of split FEC techniques (i.e. with two separated FEC) at the physical and upper layers (i.e. BB-iFEC and UL-FEC respectively). Our investigations reveal that a split FEC scheme at the physical layer like BB-iFEC not only outperforms UL-FEC techniques, but can also provide a good compromise between time diversity and zapping time compared to single FEC configurations.

Regarding the investigation on frequency diversity, we have focused on the gains that can be achieved by extending the interleaving across hundreds of $\mathrm{MHz}$ with TFS. According to the simulations, gains of around $6 \mathrm{~dB}$ are realistic in DVB-NGH depending on the code rate. Similarly to the case of long TI, the gain achieved with TFS is conditioned by the error-correcting capabilities of the FEC code, especially when TFS is performed across a higher number of RF channels.

For space diversity, we have evaluated the performance of the MIMO schemes adopted in DVB-NGH by taking into account the amount of capacity required for channel sampling (i.e. pilot information), and the error-correcting capabilities of the FEC code. The simulation results show that rate 1 MIMO schemes (eSFN and the Alamouti code) provide gains up to $6 \mathrm{~dB}$ at low CNR values mainly because of better protection against rotation and asymmetry impairments. At high CNR values, the rate 2 MIMO scheme adopted in the standard (eSM) provides gains up to $10 \mathrm{~dB}$ by doubling the multiplexing rate of information across the antennas. In the case of rate 1 MIMO, a simple technique like eSFN can outperform more complex schemes with a priori better properties like the Alamouti code if the transmission of pilots is taken into account. In the case of rate 2 MIMO, techniques based on spatial multiplexing like eSM can match and even surpass the performance of DMT-achieving schemes like the Golden code, provided that the rate of the FEC code is low enough.

Regarding the combined use of diversity, we have focused on the combination of time and frequency diversity by means of long TI and TFS, and also on the combination of time and space diversity by means of long TI and crosspolar MIMO. According to physical layer simulations performed with long TI and TFS, the utilization of one technique diminishes the gain that can be achieved with the other, so that the total gain of combining both techniques is lower than the sum of the individual gains. On the other hand, the influence of MIMO in the performance gain of long TI is more limited, and in this case, the total gain is approximately equal to the sum of the separate gains. This is in line with the information-theoretic results presented in Chapter 3. 
In parallel to long TI, TFS, and MIMO, we have investigated the use of rotated constellations as a manner to improve the performance of diversity techniques. In particular, the better protection of rotated constellations against fading and erasures is very valuable in the context of long TI and TFS. According to the simulation results, the use of rotated constellations in DVB-NGH increases the gains of long TI and TFS with code rates as low as $1 / 3$. The gain grows larger with UL interleaving, and when the interleaving extends further in the time and frequency domains. Furthermore, the additional protection of rotated constellations against the complete loss of information (i.e. erasures), can reduce the zapping time in several seconds. The results presented in this chapter also show that $4 \mathrm{DRC}$ outperform $2 \mathrm{DRC}$ for code rates higher than $1 / 2$, and that the advantage of $4 \mathrm{DRC}$ over $2 \mathrm{DRC}$ depends on the interleaving duration, the type of interleaving, and also on the number of RF channels used in TFS. More precisely, the improvement of $4 \mathrm{DRC}$ over $2 \mathrm{DRC}$ is larger with interleaving durations of several seconds, uniform interleaving, and when the number of RF channels used in TFS is four or more. 


\section{Chapter 6}

\section{Design Guidelines for Mobile Broadcasting Systems and Conclusions}

\subsection{Concluding Remarks}

This dissertation has investigated the combined use of time, frequency and space in mobile broadcasting systems, and more specifically, in the European family of standards developed by the DVB project. On one hand, information theory has been used to obtain the potential gain of diversity techniques in mobile broadcasting systems, and to help understanding the theoretical limits. On the other hand, full-featured simulators have been used to obtain a more accurate depiction of the performance achieved in DVB systems, including DVB-T, DVB-H, DVB-T2, and DVB-NGH.

\subsubsection{Time Diversity}

In the time domain, diversity is achieved by distributing the codewords over extended periods of time by means of TI. The main drawback of TI is an increase of the memory requirements and the zapping time, which are generally proportional to the interleaving duration. The information-theoretic results indicate that interleaving durations in the order of hundreds of milliseconds are sufficient to compensate for fast fading, achieving gains of around $5 \mathrm{~dB}$. Interleaving durations up to several seconds provide additional protection against 


\section{CHAPTER 6. DESIGN GUIDELINES AND CONCLUSIONS}

shadowing, and in terrestrial scenarios, the gain can be as high as $12 \mathrm{~dB}$ provided that the interleaving duration is high enough.

In real systems, the performance of TI depends on whether the FEC is implemented at the physical or upper layers. Generally speaking, PL-FEC provides better performance at the expense of higher memory requirements, whereas UL-FEC sacrifices performance in order to reduce the memory that is needed for interleaving purposes. Moreover, UL-FEC mechanisms reduce the system capacity due to the transmission of additional parity data and are only useful in mobile reception conditions. Our simulations show that for interleaving durations below $1 \mathrm{~s}$, the use of UL-FEC protection does not improve the overall spectral efficiency compared to using a more robust configuration at the physical layer. On the contrary, the use of UL-FEC with interleaving durations up to $10 \mathrm{~s}$ might improve the overall spectral efficiency depending on the velocity and the route of the users across the service area.

In addition to the interleaving duration, the distribution of information over time is also important from a time diversity point of view. On one hand, there is a trade-off between time diversity and the power saving that is possible in receivers. While the maximum time diversity is obtained when the information is transmitted continuously over time, time slicing improves the power saving capabilities of receivers by transmitting the information in a discontinuous manner. The results presented in this dissertation show that a more continuous transmission of information over time is needed for high user velocities, whereas for low velocities, this is not significant for time diversity. Another trade-off exists between time diversity and the zapping time that is perceived by the user. Although the zapping time is generally proportional to the interleaving duration, fast zapping techniques can be used to reduce the zapping time down to tolerable values. The main idea of fast zapping is to start decoding the codewords before receiving all the data and parity information. In the case of CI, UL configurations can be used to improve fast zapping at the expense of worse time diversity. Nevertheless, the utilization of fast zapping with a single FEC might result in a performance loss depending on the robustness of the FEC code with heavy puncturing. The investigations performed in the context of DVB-NGH have shown that a split FEC approach at the physical layer outperforms UL-FEC techniques and can provide a good compromise in terms of time diversity and zapping time compared to single FEC.

\subsubsection{Frequency Diversity}

Terrestrial mobile broadcasting systems achieve frequency diversity by means of COFDM. In this case, interleaving is used to distribute the FEC codewords across the active carriers of the OFDM symbols so that each codeword covers 
the entire transmission bandwidth. If the interleaving is limited to one RF channel, the gain of frequency diversity comes from better robustness against multipath fading. If the interleaving extends over multiple RF channels and across hundreds of $\mathrm{MHz}$, then it is possible to compensate for the signal variations that exist between different RF channels in the UHF band. These are caused by the frequency dependency of the transmitter site, the receivers and the propagation channel.

The mobile broadcasting systems standardized by the DVB generally achieve sufficient frequency diversity within the $8 \mathrm{MHz}$ channels of the UHF band. For this reason, the investigation on frequency diversity has centered on TFS, and particularly, on the gains that can be achieved by extending the frequency interleaving over multiple RF channels. In this dissertation, we have shown that the gain of TFS depends mostly on the extent of interleaving in the frequency domain, and that the number of RF channels does not affect the performance in an important manner. The information-theoretic results and the physical layer simulations show that very significant gains above $6 \mathrm{~dB}$ are feasible for realistic configurations of TFS in the UHF band. On the other hand, the total gain of combining long TI with TFS is lower than the sum of the individual gains due to the fact that both techniques improve the reception robustness against fast fading and shadowing.

\subsubsection{Space Diversity}

The incorporation of MIMO in mobile broadcasting systems is key to overcome the Shannon limit of single antenna communications. MIMO techniques employ multiples antennas to improve the reception robustness (i.e. diversity gain) and to increase the transmission rate of information (i.e. multiplexing gain). While the use of co-polarized antennas for MIMO purposes is well suited to vehicular receivers, the required separation between antennas in the UHF band makes them impractical for handheld devices, and in this case, cross-polarized antennas are generally preferred. The results presented in this dissertation show that SIMO and MIMO configurations provide significant gains with both co-polarized and cross-polarized antennas, whereas the gain of MISO configurations is much more limited due to the lack of CSI at the transmitter site. In particular, the utilization of cross-polar MISO has very little applicability in broadcasting systems, as it might provide a lower performance than single antenna configurations.

The potential gain of MIMO techniques with multiplexing capabilities over MIMO techniques based entirely on diversity depends on the CNR region. In the case of DVB-NGH, the gain of rate 1 MIMO schemes (eSFN and the Alamouti code) is around $6 \mathrm{~dB}$, whereas the gain of rate 2 MIMO schemes 


\section{CHAPTER 6. DESIGN GUIDELINES AND CONCLUSIONS}

(eSM) can be as high as $10 \mathrm{~dB}$ at high spectral efficiencies (6 bpc). According to our investigations, the amount of capacity required for the transmission of pilots is an important factor to consider when evaluating the actual gain of MIMO techniques, especially in mobile scenarios. In this manner, the improvement of the Alamouti code in terms of spectral efficiency is halved compared to the gain that is obtained when the transmission of pilots is not taken into account. In addition to the transmission of pilots, it is important to consider the errorcorrection capabilities of the FEC code when evaluating the performance of MIMO techniques. For example, a simple rate 1 MIMO technique such as eSFN loses less than $0.5 \mathrm{~dB}$ compared to the Alamouti code and provides an overall better spectral efficiency if the FEC code and the transmission of pilots is taken into account. Similarly, rate 2 MIMO techniques based on spatial multiplexing approach the performance of DMT-achieving schemes like the Golden code, provided that the rate of the FEC code is low enough.

The utilization of TI reduces the performance of co-polar MIMO and vice versa, as both techniques provide better protection against multipath fading. While the impact of MIMO techniques in the performance of TI is significant for interleaving durations below $1 \mathrm{~s}$, it is lower with interleaving durations up to several seconds, as multiple antennas cannot compensate for shadowing in the space domain. In contrast, the gain of cross-polar MIMO is mostly due to better robustness against rotation and asymmetry impairments. Consequently, the performance of TI and cross-polar MIMO does not depend on each other, and the gain of combining both techniques is approximately equal to the sum of the individual gains.

\subsubsection{Rotated Constellations}

Rotated constellations increase the diversity of BICM systems at the expense of additional complexity when demodulating the signal. The results based on information theory show that rotated constellations improve the performance when the code rate is high (e.g. $>1 / 2$ with QPSK), whereas for low code rates, non-rotated constellations achieve better results. While the physical layer simulations corroborate the information-theoretic results in the case of fast fading, they show that rotated constellations provide additional gains with long TI and TFS even for code rates as low as $1 / 3$. Furthermore, the additional protection of rotated constellations against the complete loss of information (i.e. erasures), can reduce the zapping time in several seconds. 


\subsection{Recommendations for the Utilization of Di- versity in Terrestrial Mobile Broadcasting Systems}

\subsubsection{Time Diversity}

The next generation of mobile broadcasting systems should prioritize the use of diversity at the physical layer in order to achieve the highest performance. UL-FEC mechanisms have been traditionally used in DVB standards to provide time diversity with reduced complexity. However, the costs associated to the implementation of TI memory have been decreasing over the years, and today, long TI can be performed at the physical layer by means of an external memory. Nonetheless, the utilization of UL-FEC is still advantageous for file delivery services, as in this case, the amount of TI memory required at the physical layer might be cost-prohibitive. Furthermore, recent advances in erasure codes, such as the development of RaptorQ codes, allow UL-FEC decoding to be done in generic software processor, even for very long interleaving durations.

While BI is practical for interleaving durations ranging hundreds of milliseconds, CI is preferable for long TI because of the advantages in terms of memory, latency, and zapping time. Due to the lowering costs of memory over time, a single FEC solution based on CI and LDPC or turbo codes should be the preferred option for long TI in future standards. However, at present, a split FEC approach at the physical layer such as BB-iFEC might be considered, as it allows for a more efficient utilization of the TI memory, while offering a good compromise between robustness and zapping performance.

Long TI requires the utilization of fast zapping to provide tolerable zapping times from the user's perspective. In the case of single FEC with CI, UL configurations must be incorporated to enable fast zapping. Depending on the FEC code, the utilization of rotated constellations is necessary to avoid an important performance degradation with UL interleaving. While turbo codes are naturally resilient to puncturing and work well with UL interleaving, this not the case of LDPC codes, for which the presence of erasures may compromise the system performance.

Together with TI, time slicing should be implemented at the physical layer in order to enable power saving at the receiver side. Despite the development of more durable batteries in the recent years, power consumption is still a serious concern in handheld devices. On the contrary, for a significant portion of mobile TV receivers such as netbooks or in-car receivers, battery lifetime is of less relevance. Therefore, it is advisable to incorporate some degree of flexibility in the configuration of time slicing, so that this can be particularized to the 


\section{CHAPTER 6. DESIGN GUIDELINES AND CONCLUSIONS}

receiver characteristics. In this manner, services targeting handheld receivers should be transmitted in a discontinuous manner for power saving, whereas services targeting vehicular receivers should be transmitted continuously over time for better diversity.

\subsubsection{Frequency Diversity}

The incorporation of TFS is highly recommendable in mobile broadcasting systems. TFS improves the reception robustness as a result of better frequency diversity and also the system capacity due to more efficient StatMux. In order to maximize the diversity gain of TFS, the separation between the lowest and the highest RF channel in the same TFS multiplex should be as large as possible. After the release of the $800 \mathrm{MHz}$ band in many European countries (the so-called digital dividend), the largest separation that can be achieved in the UHF band is approximately $300 \mathrm{MHz}$, which is sufficient to achieve gains beyond $6 \mathrm{~dB}$. On the other hand, the interference created by mobile communications (i.e. cellular systems) in the $800 \mathrm{MHz}$ band is expected to jeopardize the reception of broadcasting systems, especially in the case of portable and mobile receivers. In this context, the utilization of TFS can be very useful to protect broadcasting services being transmitted in the UHF band from the adjacent-band interference caused by cellular networks.

\subsubsection{Space Diversity}

Next generation systems should mandate or at least encourage the implementation of multiple antennas in receivers. This provides very large gains with cross-polarized or co-polarized antennas even if the transmitters are equipped with a single antenna. While a pair of cross-polarized antennas is better suited to handheld devices at UHF frequencies, multiple co-polarized antennas can be easily fitted in vehicular receivers. In contrast, the implementation of multiple antennas in transmitters for MISO purposes is not recommended in mobile broadcasting systems. The performance of MISO techniques in mobile scenarios is limited, and generally, it does not justify the implementation of multiple transmit antennas. Moreover, MISO systems based on cross-polarized antennas may actually result in a lower performance than their SISO counterparts. Distributed MISO techniques are still recommended, as in this case, better diversity is achieved without any modification in the existent network infrastructure. Standard compatible schemes like eSFN are preferable over SFBCs like the Alamouti code due to the lower number of pilots that must be transmitted for channel estimation. This not only provides a higher performance in 
terms of spectral efficiency, but it might also allow for better robustness against channel estimation errors.

For MIMO systems, rate 2 schemes based on spatial multiplexing outperform rate 1 schemes for every configuration of MODCOD. However, the additional complexity required for demodulating rate 2 MIMO schemes and the mandatory implementation of multiple antennas in receivers, justifies their utilization only at high spectral efficiencies $(>2 \mathrm{bpc}$ ), for which the improvement over rate 1 MIMO schemes is significantly larger. In the case of rate 2 MIMO, schemes based on spatial multiplexing like eSM are advisable, since they achieve the same performance as more sophisticated schemes like the Golden code, but with much lower complexity.

The incorporation of rate 1 and rate 2 MIMO schemes is very interesting in the context of Scalable Video Coding (SVC) [113], which is expected to play a significant role in next generation systems. The SVC extension of H.264/AVC allows for extracting different video representations from a single bitstream, where the different substreams are referred to as layers. The base layer provides the lowest level of quality and ensures backwards compatibility with H.264/AVC compliant receivers. Additional enhancement layers improve the video quality of the base layer in the temporal, spatial or quality dimension. The main idea is to transmit the base and enhancement layers of the same video service as separated components with specific robustness. On one hand, the base layer is transmitted in a manner to ensure reception in the entire coverage area and for all receivers. To do so, the transmitted signal is modulated with low order constellations, more robust code rates and rate 1 MIMO, which allows compatibility with single antenna receivers. On the other hand, the enhancement layers target users in good reception conditions (e.g. outdoor scenarios) or with better equipment (e.g. higher gain and/or multiple antennas), which can enjoy a better video quality. In this case, the transmitted signal is transmitted with more relaxed parameters and rate 2 MIMO schemes in order to benefit from the multiplexing gain in the high CNR region.

\subsubsection{Rotated Constellations}

Rotated constellations should be the norm in the next generation of mobile broadcasting systems for low order constellations such as QPSK. The better protection against fading and erasures outweighs the slight performance degradation that might occur at low code rates. In particular, the inclusion of rotated constellations is very valuable to improve the reception robustness with long TI and TFS, and also to reduce the zapping time. Together with rotated constellations, it is important that the component interleaver is optimized according to the distribution of information over time and frequency. 


\section{CHAPTER 6. DESIGN GUIDELINES AND CONCLUSIONS}

Ideally, the interleaver should ensure the maximum possible separation between components in the time and frequency domains.

\subsection{Future Research Topics}

Iterative decoding has been proposed in BICM systems to reduce the performance gap with CM and approach the channel capacity in AWGN [114]. This is usually referred to as Bit Interleaved Coded Modulation with Iterative Decoding (BICM-ID). The principle of BICM-ID is to exchange soft information between the FEC decoder and the symbol demodulator in an iterative manner as it is shown in Fig. 6.1. Here, the demodulator and the FEC decoder generate extrinsic information in LLR form, which after passing through the deinterleaver $\pi^{-1}$ and the interleaver $\pi$, become the a priori information of the decoder and the demodulator respectively. The a posteriori LLRs generated by the FEC decoder can be used to make hard decision after each iteration. Despite the fact that, at present, iterative techniques may not be practical in real receivers due to implementation costs, future receivers can incorporate BICM-ID architectures in order to benefit from an important performance increase with no modification required in standards or transmitters.

The diversity order of BICM-ID and hence, its performance in the presence of fading, can be further improved by means of rotated constellations $[115,116]$. In the context of DVB-T2, it has been shown that the utilization of iterative decoding with rotated constellations can provide gains of several dBs in fading channels [81]. Iterative techniques can also be used to approach the capacity of the MIMO channel in BICM systems with multiple antennas [117]. Combining iterative decoding with MIMO is particularly challenging, as the decoding complexity of MIMO signals grows exponentially with the number of antennas. Therefore, the investigation of receiver architectures that can approximate the performance of joint detection and decoding by means of iterative techniques, while maintaining the complexity in affordable levels with a large number of antennas, is a very interesting topic.

Channel estimation is an important topic in the context of MIMO. On one hand, we have shown that the amount of pilot information that is required for channel estimation is determinant for the performance gains of MIMO techniques in mobile broadcasting systems. On the other hand, while the work presented in this dissertation has assumed ideal channel estimation, the performance of MIMO techniques might be conditioned by the quality of the channel estimates [77]. In this sense, it would be very interesting to evaluate the impact of real channel estimation, and to devise new pilot patterns optimized for MIMO systems in terms of reception robustness and pilot overhead. 


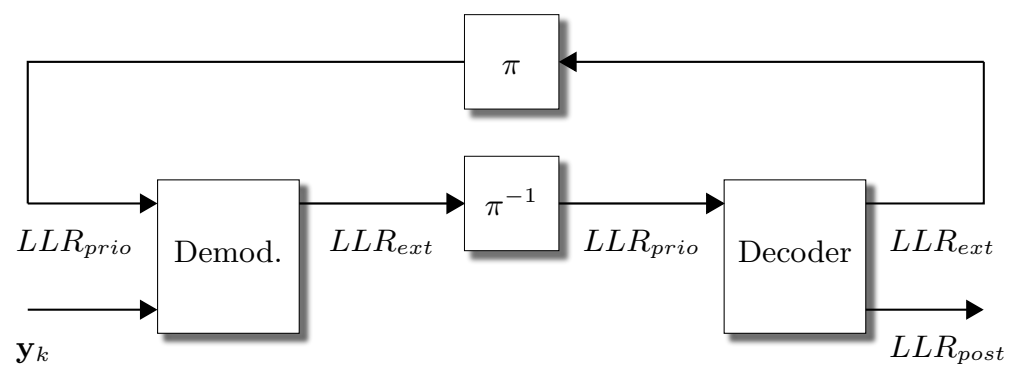

Figure 6.1: BICM-ID receiver.

Another important subject is related to the utilization of long TI in mobile broadcasting systems. In this thesis, we have computed the gains of long TI from the point of view of better protection against fast fading and shadowing by assuming that the user is moving at constant velocity across the service area. In practice, the users may become temporarily stationary due to traffic lights for example. The gain of TI for moderate to long interleaving durations is much more reduced in stationary conditions, as in this case, the gain stems only from the dynamic variations in the surroundings of the receiver. In order to evaluate the actual advantage of long TI in terms of network planning, it is necessary to perform dynamic system level simulations that take into account the user mobility in a realistic manner [106]. While this kind of investigations has been already performed in the context of DVB-T and DVB-H networks [118], it has yet to be extended to the case of DVB-T2 and DVB-NGH. Here, it is interesting to evaluate the network gains of long $\mathrm{TI}$ at the physical layer in combination with TFS and MIMO, and to determine whether the introduction of DVB-NGH transmissions can enable the provision of mobile services with good coverage levels in DVB-T2 networks planned for fixed reception. 


\section{References}

[1] Framing structure, channel coding and modulation for digital terrestrial television, ETSI Std. EN 300 744, Rev. 1.6.1, 2009.

[2] "DVB-T - digital terrestrial television," DVB Fact Sheet, 2010.

[3] E. Pinson, "Coming next: 3D television over DVB-T2," White Paper, Teamcast, 2010.

[4] Frame structure channel coding and modulation for a second generation digital terrestrial television broadcasting system (DVB-T2), ETSI Std. EN 302 755, Rev. 1.2.1, 2011.

[5] "DVB-T2 - 2nd generation terrestrial broadcasting," DVB Fact Sheet, 2010.

[6] Transmission system for handheld terminals, ETSI Std. EN 302 304, Rev. 1.1.1, 2004.

[7] Framing Structure, channel coding and modulation for Satellite Services to Handheld devices (SH) below $3 \mathrm{GHz}$, ETSI Std. EN 302 583, Rev. 1.1.2, 2010 .

[8] A. Mimnaugh et al., "Enabling mobile coverage for DVB-T," White Paper, Digital Fountain, 2009.

[9] A. Bria and D. Gómez-Barquero, "Scalability of DVB-H deployment on existing wireless infrastructure," in IEEE International Symposium on Personal, Indoor and Mobile Radio Communications (PIMRC'05), Berlin, Germany, Sep. 2005.

[10] D. Gómez-Barquero, "Cost efficient provisioning of mass mobile multimedia services in hybrid cellular and broadcasting systems," Ph.D. dissertation, Universidad Politécnica de Valencia, 2009. 
[11] G. Pousset, Y. Lostanlen, and Y. Corre, "Positioning mobile TV standards DVB-T vs DVB-H," White Paper, Dibcom, 2008.

[12] F. Selgert, "Commercial requirements for DVB-NGH," DVB Document CM-NGH015R1, 2009.

[13] S. Lin and D. J. Costello, Error Control Coding, 2nd ed. Prentice Hall, 2004.

[14] C. E. Shannon, "A mathematical theory of communication," Bell System Technical Journal, vol. 27, pp. 379-423, 623-656, 1948.

[15] M. Eroz, L. N. Lee, and F. W. Sun, "Impact of near shannon-limit codes on wireless communications technology," in IEEE International Symposium on Personal, Indoor and Mobile Radio Communications (PIMRC'04), Barcelona, Spain, Sep. 2004.

[16] D. J. C. Mackay and R. M. Neal, "Near shannon limit performance of low density parity check codes," IEEE Electronic Letters, vol. 33, no. 6, pp. 457-458, 1996.

[17] C. Berrou, A. Glavieux, and P. Thitimajshima, "Near shannon limit error-correcting coding and decoding: Turbo-codes," in IEEE International Conference on Communications (ICC'93), vol. 2, Geneva, Switzerland, May 1993, pp. 1064-1070.

[18] M. Eroz, F. W. Sun, and L. N. Lee, "DVB-S2 low density parity check codes with near shannon limit performance," International Journal of Satellite Communications and Networking, vol. 22, no. 3, pp. 269-279, 2004.

[19] S. Saunders and A. Aragón-Zabala, Antennas and Propagation for Wireless Communication Systems, 2nd ed. Wiley, 2007.

[20] J. D. Parsons, The Mobile Radio Propagation Channel. Wiley, 2000.

[21] L. Litwin and M. Pugel, "The principles of OFDM," RF Design, no. 1, pp. 30-48, 2001.

[22] R. van Nee and R. Prasad, OFDM for Wireless Multimedia Communications. Artech House Publishers, 2000.

[23] E. Huuhka et al., "DVB-T2 network planning," B21C, Deliverable D11 Part 3, Nov. 2009. 
[24] D. Tse and P. Viswanath, Fundamentals of Wireless Communications. Cambridge University Press, 2005.

[25] J. Boutros and E. Viterbo, "Signal space diversity: A power and bandwidth efficient diversity technique for the rayleigh fading channel," IEEE Transactions on Information Theory, vol. 44, no. 4, pp. 1453-1467, 1998.

[26] J. Ramsey, "Realization of optimum interleavers," IEEE Transactions on Information Theory, vol. 16, no. 3, pp. 338-345, 1970.

[27] Upper Layer Forward Error Correction for DVB Systems (ULFEC), ETSI Std. TS 102 993, Rev. 1.1.1, 2011.

[28] H. Fuchs and N. Farber, "Optimizing channel change time in IPTV applications," in IEEE Broadband Multimedia Systems and Broadcasting (BMSB'08), Las Vegas, USA, Mar. 2008.

[29] A. J. Paulraj, D. A. Gore, R. U. Nabar, and H. Bolcskei, "An overview of MIMO communications - A key to gigabit wireless," Proceedings of the IEEE, vol. 92, no. 2, pp. 198-218, 2004.

[30] R. U. Nabar, H. Bolcskei, V. Erceg, D. Gesbert, and A. J. Paulraj, "Performance of multiantenna signalling techniques in the presence of polarization diversity," IEEE Transactions on Signal Processing, vol. 50, no. 10, pp. 2553-2562, 2002.

[31] S. M. Alamouti, "A simple transmit diversity technique for wireless communications," IEEE Journal on Selected Areas in Communications, vol. 16, no. 8, pp. 1451-1458, 1998.

[32] E. Telatar, "Capacity of multi-antenna gaussian channels," European Transactions on Telecommunications, vol. 10, no. 6, pp. 585-596, 1999.

[33] S. Kaiser, "Spatial transmit diversity techniques for broadband ofdm systems," in IEEE Global Telecommunications Conference (GLOBECOM'00), vol. 3, San Francisco, USA, Aug. 2000, pp. 1824-1828.

[34] A. Dammann and S. Kaiser, "Standard conformable antenna diversity techniques for OFDM and its application to the DVB-T system," in IEEE Global Telecommunications Conference (GLOBECOM'01), vol. 5, San Antonio, USA, Nov. 2001, pp. 3100-3105.

[35] R. Burrow et al., "On the performance of the DVB-T system in mobile environments," in IEEE Global Telecommunications Conference (GLOBECOM'98), Sidney, Australia, Nov. 1998. 
[36] M. Poggioni, L. Rugini, and P. Banelli, "DVB-T/H and T-DMB: Physical layer performance comparisons in fast mobile channels," IEEE Transactions on Broadcasting, vol. 55, no. 4, pp. 719-730, 2009.

[37] Implementation guidelines for DVB handheld services, ETSI Std. TR 102 377, Rev. 1.4.1, 2009.

[38] D. Plets et al., "Influence of reception condition, MPE-FEC rate and modulation scheme on performance of DVB-H," IEEE Transactions on Broadcasting, vol. 54, no. 3, pp. 590-598, 2008.

[39] G. Berjon-Eriz et al., "Laboratory tests for testing DVB-T2 mobile performance," in IEEE Broadband Multimedia Systems and Broadcasting (BMSB'10), Shanghai, China, Mar. 2010.

[40] T. Jokela and J. Paavola, "Robustness analysis of physical layer signaling transmission in DVB-T2," in IEEE Broadband Multimedia Systems and Broadcasting (BMSB'09), Bilbao, Spain, May 2009.

[41] T. Jokela, M. Tupala, and J. Paavola, "Analysis of physical layer signaling transmission in DVB-T2 systems," IEEE Transactions on Broadcasting, vol. 56, no. 3, pp. 410-417, 2010.

[42] Implementation guidelines for a second generation digital terrestrial television broadcasting system (DVB-T2), ETSI Std. TR 102 831, Rev. $0.10 .4,2010$.

[43] Guidelines for Implementation for Satellite Services to Handheld devices (SH) below 3GHz, ETSI Std. TS 102 584, Rev. 1.2.1, 2011.

[44] M. Cohen et al., "DVB-SH field trial SFN and diversity gain measurements," in European Conference on Antennas and Propagation (EuCAP'09), Berlin, Germany, Mar. 2009.

[45] A. Heuberger, H. Stadali, and S. Sudler, "Field testing of a DVB-SH waveform in Japan," in IEEE Broadband Multimedia Systems and Broadcasting (BMSB'09), Bilbao, Spain, May 2009.

[46] D. Gómez-Barquero, D. Gozálvez, and N. Cardona, "Application layer FEC for mobile TV delivery in IP datacast over DVB-H systems," IEEE Transactions on Broadcasting, vol. 55, no. 2, pp. 396-406, 2009.

[47] D. Gómez-Barquero and A. Bria, "Application Layer FEC for improved mobile reception of DVB-H streaming services," in IEEE Vehicular Technology Conference (VTC'06), Montreal, Canada, Sep. 2006. 
[48] IP Datacast over DVB-H: Content Delivery Protocols, ETSI Std. TS 102 472, Rev. 1.3.1, 2009.

[49] A. Shokrollahi, "Raptor codes," IEEE Transactions on Information Theory, vol. 52, no. 6, pp. 2551-2567, 2006.

[50] M. Makni, J. Robert, and E. Stare, "Performance analysis of time frequency slicing," in ITG Conference on Electronic Media Technology (CEMT'11), Dortmund, Germany, Mar. 2011.

[51] S. Bergsmark, "Considerations on time-frequency slicing coverage gain based on field measurements of received signal strength," DVB Technical Module, TM-T20238, Sep. 2007.

[52] G. Pousset, "Advantages of diversity implementations on mobile and portable TV receivers," White Paper, Dibcom, 2008.

[53] U. Ladebusch and C. A. Liss, "Terrestrial DVB (DVB-T): A broadcast technology for stationary, portable and mobile use," Proceedings of the IEEE, vol. 94, no. 1, pp. 183-193, 2006.

[54] R. D. Bari et al., "Laboratory measurement campaign of DVB-T signal with transmit delay diversity," IEEE Transactions on Broadcasting, vol. 54, no. 3, pp. 532-541, 2008.

[55] Y. Zhang, J. Cosmas, M. Bard, and Y. Song, "Diversity gain for DVB-H by using transmitter/receiver cyclic delay diversity," IEEE Transactions on Broadcasting, vol. 52, no. 4, pp. 464-474, 2006.

[56] Y. Zhang, J. Cosmas, K.-K. Loo, M. Bard, and R. D. Bari, "Analysis of cyclic delay diversity on DVB-H systems over spatially correlated channel," IEEE Transactions on Broadcasting, vol. 53, no. 1, pp. 247-254, 2007.

[57] N. Cornillet, M. Crussiere, and J. F. Helard, "Performance of the DVB-T2 system in a single frequency network: Analysis of the distributed Alamouti scheme," in IEEE Broadband Multimedia Systems and Broadcasting (BMSB'11), Nuremberg, Germany, Jun. 2011.

[58] M. Tormos, C. Tanougast, P. Kasser, P. Bretillon, and A. Dandache, "Performance evaluation for different MISO - SFN network combinations for DVB-T2 standard," in IEEE Broadband Multimedia Systems and Broadcasting (BMSB'11), Nuremberg, Germany, Jun. 2011. 
[59] P. Antanes et al., "MIMO performance of the next generation DVB-T," in IEEE Vehicular Technology Conference (VTC'09), Barcelona, Spain, Apr. 2009.

[60] C. Gómez-Calero, L. C. Navarrete, L. de Haro, and R. Martínez, "A 2x2 MIMO DVB-T2 system: Design, new channel estimation scheme and measurements with polarization diversity," IEEE Transactions on Broadcasting, vol. 56, no. 2, pp. 184-192, 2010.

[61] L. H. Ozarow, S. Shamai, and A. D. Wyner, "Information theoretic considerations for cellular mobile radio," IEEE Transactions on Vehicular Technology, vol. 43, no. 2, pp. 359-378, 1994.

[62] A. Goldsmith, Wireless Communications. Cambridge University Press, 2005.

[63] G. Ungerboeck, "Channel coding with multilevel/phase signals," IEEE Transactions on Information Theory, vol. 28, no. 1, pp. 56-67, 1982.

[64] G. Caire, G. Taricco, and E. Biglieri, "Bit-interleaved coded modulation," IEEE Transactions on Information Theory, vol. 44, no. 3, pp. 927-946, 1998.

[65] I. S. Reed and G. Solomon, "Polynomial codes over certain fields," Journal of the Society for Industrial and Applied Mathematics, no. 8, pp. 300-304, 1960.

[66] A. Shockrollahi, "Raptor codes," IEEE Transactions on Information Theory, vol. 52, no. 6, pp. 2551-2567, 2006.

[67] D. J. C. Mackay, "Fountain codes," IEE Proceedings Communications, vol. 152, no. 6, pp. 1062-1068, 2005.

[68] M. Luby, A. Shockrollahi, M. Watson, T. Stockhammer, and L. Minder, "RaptorQ forward error correction scheme for object delivery," IETF, RFC 6330, May 2011.

[69] "RaptorQ," Technical Overview, Qualcomm, 2010.

[70] C. Berrou, R. Pyndiah, P. Adde, C. Douillard, and R. L. Bidan, "An overview of turbo codes and their applications," in The European Conference on Wireless Technology, Nicosia, Cyprus, Oct. 2005.

[71] R. G. Gallager, "Low-density parity-check codes," IRE Trans. Inform. Theory, vol. 8, no. 1, pp. 21-28, 1962. 
[72] R. McEliece, D. J. C. Mackay, and J. F. Cheng, "Turbo decoding as an instance of Pearl's belief propagation algorithm," IEEE Journal on Selected Areas in Communications, vol. 16, no. 2, pp. 140-152, 1998.

[73] G. Forney, "Burst-correcting codes for the classic bursty channel," IEEE Transactions on Circuit Theory, vol. 19, no. 5, pp. 772-781, 1971.

[74] E. Biglieri, MIMO Wireless Communications. Cambridge University Press, 2007.

[75] A. Lozano and N. Jindal, "Transmit diversity vs. spatial multiplexing in modern MIMO systems," IEEE Transactions on Wireless Communications, vol. 9, no. 1, pp. 186-197, 2010.

[76] J. C. Belfiore, G. Rekaya, and E. Viterbo, "The golden code: a $2 \times 2$ fullrate space-time code with non-vanishing determinants," in International Symposium on Information Theory (ISIT'04), Chicago, USA, Jul. 2004.

[77] P. Fertl, "Performance limits and channel state information in wireless MIMO communication systems," Ph.D. dissertation, Technischen Universitat Wien, 2009.

[78] B. Hassibi and H. Vikalo, "On the sphere-decoding algorithm i. expected complexity," IEEE Transactions on Signal Processing, vol. 53, no. 8, pp. $2806-2818,2005$.

[79] M. O. Damen, H. E. Gamal, and G. Caire, "On maximum-likelihood detection and the search for the closest lattice point," IEEE Transactions on Information Theory, vol. 49, no. 10, pp. 2389-2402, 2003.

[80] A. Paulraj, R. Nabar, and D. Gore, Introduction to Space-Time Wireless Communications. Cambridge University Press, 2003.

[81] C. A. Nour and C. Douillard, "Rotated QAM constellations to improve BICM performance for DVB-T2," in IEEE International Symposium on Spread Spectrum Techniques and Applications (ISSSTA'08), Bologna, Italy, Aug. 2008.

[82] S. P. Herath, "Rotated multi-D constellations in Rayleigh fading: Mutual information improvement and a pragmatic approach for near-capacity performance in high-rate regions," in IEEE Wireless Communications and Networking Conference (WCNC'11), Cancun, Mexico, Mar. 2011.

[83] T. Rahrer, R. Fiandra, S. Wright, D. Allan, and D. Thorne, "Triple-play services quality of experience (QoE) requirements," DSL Forum, TR-126, Dec. 2006. 
[84] R. Kooij, K. Ahmed, and K. Brunnström, "Perceived quality of channel zapping," in International Conference Communication Systems and Networks (CSN'06), Palma de Mallorca, Spain, Aug. 2006.

[85] M. L. A. Morel, S. Kerboeuf, B. S. adn Y. Leprovost, and F. Faucheux, "Performance evaluation of channel change for DVB-SH streaming services," in IEEE International Conference on Communications (ICC'00), Cape Town, South Africa, Jul. 2010.

[86] Specification for data broadcasting, DVB Std. EN 301 192, Rev. 1.4.2, 2008.

[87] Multimedia Broadcast/Multicast Service (MBMS); Protocols and Codecs, 3GPP Std. TS 26.346, Rev. 9.4.1, 2011.

[88] D. Gozálvez, D. Gómez-Barquero, T. Stockhammer, and M. Luby, "AL-FEC for improved mobile reception of MPEG-2 DVB-T transport streams," International Journal of Digital Multimedia Broadcasting - special issue on Convergence of Digital TV Systems and Services, 2009.

[89] M. Rezaei, I. Bouazizi, and M. Gabbouj, "Statistical time-frequency multiplexing of HD video traffic in DVB-T2," International Journal of Digital Multimedia Broadcasting, 2009.

[90] J. Robert, "Improved robustness and transmitter identification for multiantenna systems," DVB Technical Module, TM-H1164, Feb. 2011.

[91] B. Varadarajan and J. R. Barry, "The outage capacity of linear spacetime codes," IEEE Transactions on Wireless Communications, vol. 4, no. 6 , pp. 1536-1276, 2005.

[92] R. U. Nabar, H. Bolcskei, and A. J. Paulraj, "Transmit optimization for spatial multiplexing in the presence of spatial fading correlation," in IEEE Global Telecommunications Conference (GLOBECOM'01), San Antonio, USA, Nov. 2001.

[93] L. Zheng and D. N. C. Tse, "Diversity and multiplexing: A fundamental tradeoff in multiple-antenna channels," IEEE Transactions on Information Theory, vol. 49, no. 5, pp. 1073-1096, 2003.

[94] H. Bolcskei, D. Gesbert, and A. J. Paulraj, "On the capacity of OFDMbased spatial multiplexing systems," IEEE Transactions on Communications, vol. 50, no. 2, pp. 225-234, 2002. 
[95] M. Failli et al., "Digital land mobile radio communications," COST 207, Final report, Sep. 1988.

[96] "Simulation report," Deliverable D5, Wing TV, 2006.

[97] P. Moss, T. Y. Poon, and J. Boyer, "A simple model of the UHF crosspolar terrestrial channel for DVB-NGH," White Paper, BBC, 2011.

[98] F. P. Fontán, M. Vázquez-Castro, C. E. Cabado, J. P. García, and E. Kubista, "Statistical modeling of the LMS channel," IEEE Transactions on Vehicular Technology, vol. 50, no. 6, pp. 1549-1567, 2001.

[99] "Frequency and network planning aspects of DVB-T2," Technical Report, EBU Tech 3348 v. 2.0, 2012.

[100] J. J. Giménez, D. Gozálvez, D. Gómez-Barquero, and N. Cardona, "A statistical model of the signal strength imbalance between RF channels in a DTT network," Electronics Letters, 2012, to be published.

[101] P. Fertl, J. Jaldén, and G. Matz, "Performance assessment of MIMOBICM demodulators based on mutual information," IEEE Transactions on Signal Processing, vol. 60, no. 3, pp. 1366-1382, 2012.

[102] C. A. Nour and C. Douillard, "Improving BICM performance of QAM constellations for broadcasting applications," in 5th International Symposium on Turbo Codes and Related Topics, Lausanne, Switzerland, Sep. 2008.

[103] Implementation guidelines for DVB terrestrial services; Transmission aspects, ETSI Std. TR 101 190, Rev. 1.3.2, 2011.

[104] J. López-Sánchez, C. Romero, D. Gómez-Barquero, and N. Cardona, "On transmitting of global and local contents within DVB-T single frequency networks," IEEE Latin America Transactions, vol. 9, no. 4, pp. 484-491, 2011.

[105] D. Gómez-Barquero, D. Gozálvez, P. F. Gómez, and N. Cardona, "Fading margin reduction due to interburst upper layer FEC in terrestrial mobile broadcast systems," IEEE Transactions on Vehicular Technology, vol. 60, no. 7, pp. 3110-3117, 2011.

[106] D. Gómez-Barquero, P. Unger, T. Kurner, and N. Cardona, "Coverage estimation for multiburst FEC mobile TV services in DVB-H systems," IEEE Transactions on Vehicular Technology, vol. 59, no. 7, pp. 3491$3500,2010$. 
[107] M. Eroz, F. W. Sun, and L. N. Lee, "An innovative low-density paritycheck code design with near-shannon-limit performance and simple implementation," IEEE Transactions on Communications, vol. 54, no. 1, pp. 13-17, 2006.

[108] J. Ha, J. Kim, and S. W. McLaughlin, "Rate-compatible puncturing of low-density parity-check codes," IEEE Transactions on Information Theory, vol. 50, no. 11, pp. 2824-2836, 2004.

[109] C. Hsu and A. Anastasopoulos, "Capacity achieving LDPC codes through puncturing," IEEE Transactions on Information Theory, vol. 54, no. 10, pp. 4698-4706, 2008.

[110] M. Smolnikar, T. Javornik, M. Mohorcic, S. Papaharalabos, and P. T. Mathiopoulos, "Rate-compatible punctured DVB-S2 LDPC codes for DVB-SH applications," in International Workshop on Satellite and Space Communications (IWSSC'09), Siena-Tuscany, Italy, Sep. 2009.

[111] D. Gómez-Barquero, P. F. Gómez, D. Gozálvez, B. Sayadi, and L. Roullet, "A novel physical layer split fec scheme for long time interleaving with fast zapping support," IEEE Transactions on Broadcasting, 2012.

[112] B. M. Hochwald and S. Brink, "Achieving near-capacity on a multipleantenna channel," IEEE Transactions on Communications, vol. 51, no. 3, pp. 389-399, 2003.

[113] H. Schwarz, D. Marpe, and T. Wiegand, "Overview of the scalable video coding extension of the H.264/AVC standard," IEEE Transactions on Circuits and Systems for Video Technology, vol. 17, no. 9, pp. 1103-1120, 2007.

[114] X. Li, A. Chindapol, and J. A. Ritcey, "Bit-interleaved coded modulation with iterative decoding and 8PSK signaling," IEEE Transactions on Communications, vol. 50, no. 8, pp. 1250-1257, 2002.

[115] A. Chindapol and J. A. Ritcey, "Design, analysis, and performance evaluation for BICM-ID with square QAM constellations in rayleigh fading channels," IEEE Journal on Selected Areas in Communications, vol. 19, no. 5, pp. 944-957, 2001.

[116] N. H. Tran, H. H. Nguyen, and T. Le-Ngoc, "Performance of BICM-ID with signal space diversity," IEEE Transactions on Wireless Communications, vol. 6, no. 5, pp. 1732-1742, 2007. 
[117] B. M. Hochwald and S. ten Brink, "Achieving near-capacity on a multipleantenna channel," IEEE Transactions on Communications, vol. 51, no. 3, pp. 389-399, 2003.

[118] J. López-Sánchez, D. Gómez-Barquero, D. Gozálvez, and N. Cardona, "On the provisioning of mobile digital terrestrial TV services to vehicles with DVB-T," IEEE Transactions on Broadcasting, 2012, to be published. 